\title{
Electrochemical Rearrangement of 3-Hydroxyoxindoles into Benzoxazinones
}

\author{
Supporting Information
}

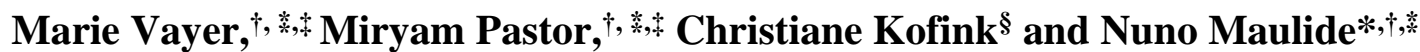

\footnotetext{
+ Institute of Organic Chemistry, University of Vienna, Währinger Strasse 38, 1090 Vienna, Austria, ${ }^{\S}$ DoktorBoehringer-Gasse 5-11, 1120 Vienna, Austria, and * Christian-Doppler Laboratory for Entropy-Oriented Drug Design, Währinger Strasse 38, 1090 Vienna, Austria.

$\$$ These authors contributed equally.

*E-mail: nuno.maulide@univie.ac.at
}

\section{Table of Contents}

1 GENERAL REMARKS

2 GENERAL PROCEDURE FOR ELECTROCHEMICAL FORMATION OF 3,1-BENZOXAZIN-2-ONES

3 EXPERIMENTAL PROCEDURES AND CHARACTERIZATION DATA OF THE STARTING MATERIALS 1, 3A-L AND 5A-G

4 CHARACTERIZATION DATA OF 3,1-BENZOXAZIN-2-ONES 2A-G, 4A-L AND 6A-F ............................. S13

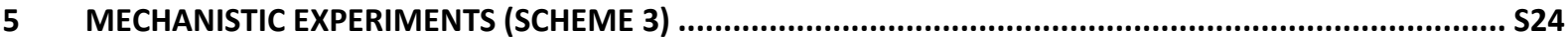

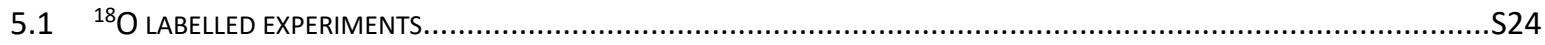

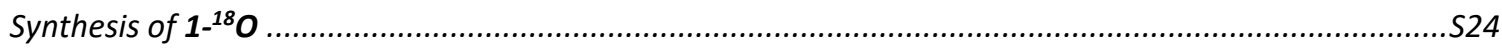

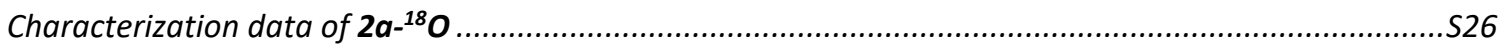

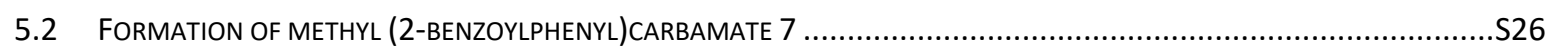

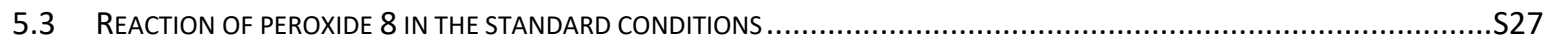

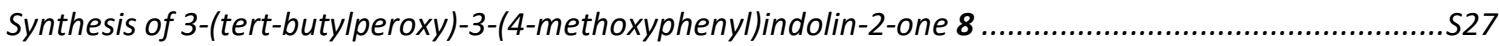

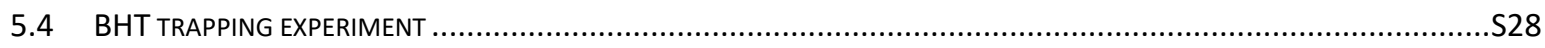

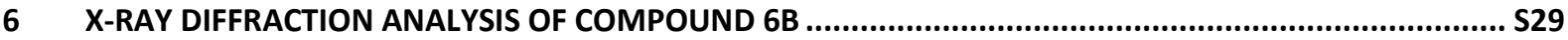

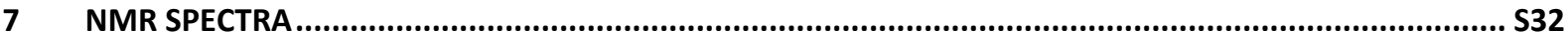

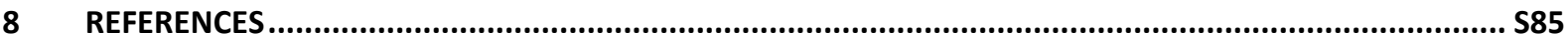




\section{General Remarks}

All solvents were distilled from appropriate drying agents prior to use or directly taken from commercial sealed bottles under an atmosphere of argon. All reagents were used as received from commercial suppliers (Alfa Aesar, Sigma Aldrich or TCI) unless otherwise stated. Reaction progress was monitored by thin layer chromatography (TLC) performed on aluminum plates coated with silica gel $\mathrm{F}_{254}$ with $0.2 \mathrm{~mm}$ thickness. Chromatograms were visualized by fluorescence quenching with UV light at $254 \mathrm{~nm}$ and/or by staining using vanilin. Flash column chromatography was performed using silica gel 60 (230-400 mesh, Merck and co.). Yields refer to chromatographically and spectroscopically pure compounds. ${ }^{1} \mathrm{H}$ NMR, ${ }^{13} \mathrm{C}$ NMR and ${ }^{18} \mathrm{~F}$ NMR spectra were recorded using a Bruker AV-400, AV-600 and AV-700 spectrometer at 300K. ${ }^{1} \mathrm{H}$ NMR chemical shifts are reported in ppm using residual solvent peak as reference $\left(\mathrm{CDCl}_{3}: \delta=7.26 \mathrm{ppm}\right.$ or DMSO-d 6 : $\left.\delta=2.50 \mathrm{ppm}\right)$. Data for ${ }^{1} \mathrm{H}$ NMR are presented as follows: chemical shift $\delta(\mathrm{ppm})$, multiplicity ( $=$ singlet, $\mathrm{d}=$ doublet, $\mathrm{t}=$ triplet, $\mathrm{m}=$ multiplet, $\mathrm{br}=$ broad), coupling constant $J(\mathrm{~Hz})$ and integration; ${ }^{13} \mathrm{C}$ NMR spectra were recorded at 100,150 or $175 \mathrm{MHz}$ using broadband proton decoupling and chemical shifts are reported in ppm using residual solvent peaks as reference $\left(\mathrm{CDCl}_{3}\right.$ : $\delta=77.16 \mathrm{ppm}$ or DMSO-d 6 : $\delta=39.52 \mathrm{ppm}$ ). Multiplicity was defined by recorded a ${ }^{13} \mathrm{C}$ NMR spectra using the attached proton test (APT). Neat infra-red spectra were recorded using a Brucker Vertex 70 FT-IR spectrometer. Wavenumbers are reported in $\mathrm{cm}^{-1}$. Mass spectra were obtained using a Finnigan MAT 8200 or $(70 \mathrm{eV})$ or an Agilent $5973(70 \mathrm{eV})$ spectrometer, using electrospray ionization (ESI) and a maXis UHR-TOF analyzer.

\section{Electrolysis general information}

Electrochemical reactions were performed with ElectraSyn 2.0 package (IKA) using the constant current mode. The reactions were conducted in a $10 \mathrm{~mL}$ vial with a magnetic stir bar and a graphite-SK-50 $(5.0 \times 0.8 \times 0.2 \mathrm{~cm})$ working electrode and a platinum-plated $(5.0 \times 0.8$ $\mathrm{x} 0.2 \mathrm{~cm}$ ) counter-electrode with a distance of $0.6 \mathrm{~cm}$ between the two electrodes. 


\section{General Procedure for Electrochemical Formation of 3,1-Benzoxazin-2-ones}

General procedure (A) to access 3,1-Benzoxazin-2-ones
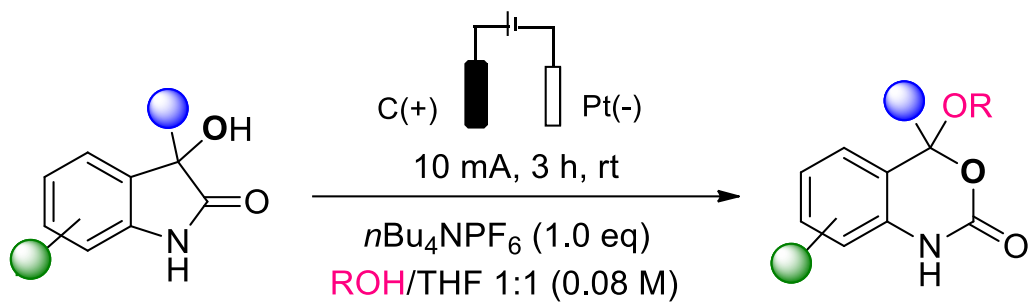

The ElectraSyn vial $(10 \mathrm{~mL})$ equipped with a stir bar was charged with 3-hydroxyoxindole derivatives 1, 3a-l, 5a-f ( $0.40 \mathrm{mmol}, 1.0$ equiv.), $n \mathrm{Bu}_{4} \mathrm{NPF}_{6}$ (155 mg, $0.40 \mathrm{mmol}, 1.0$ equiv.), $\mathrm{ROH}(2.5 \mathrm{~mL})$ and THF $(2.5 \mathrm{~mL})$. The ElectraSyn vial cap equipped with anode (graphite) and cathode (platinum) was inserted into the mixture. The reaction mixture was electrolyzed at a constant current of $10 \mathrm{~mA}$ for $3 \mathrm{~h}$. The ElectraSyn vial cap was removed, and electrodes were rinsed with $\mathrm{CH}_{2} \mathrm{Cl}_{2}(2.0 \mathrm{~mL})$, which was combined with the crude mixture. Then, the crude mixture was concentrated under reduced pressure and purified by FC over silica gel (20 $\mathrm{g} \mathrm{SiO}_{2}$, heptane/EtOAc, 100/0 to 50/50, gradient) to furnish the desired 2a-g, 4a-l, 6a-f.

Due to limitation of this specific electrochemical device, the maximum reaction limit is 0.4 mmol.

\section{Experimental Procedures and Characterization Data of the Starting Materials 1, 3a-l and 5a-g}

General procedure (B) to access 3-hydroxysusbtituted oxindoles (1, 3a-l, 5a-g)

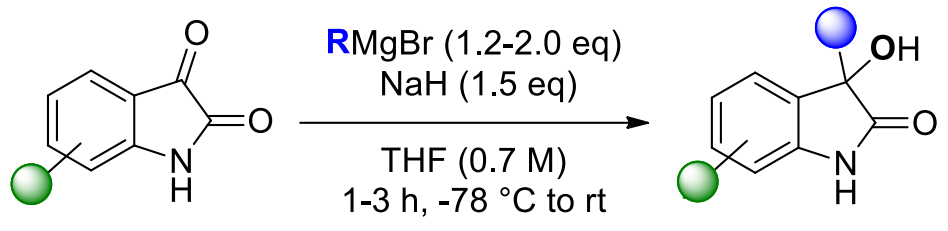

1, 3a-I, 5a-g

To a solution of the corresponding isatin derivate (1.0 equiv.) in THF $(0.7 \mathrm{M}), \mathrm{NaH}$ ( 1.5 equiv.) was added at $-78{ }^{\circ} \mathrm{C}$. The mixture was stirred for $30 \mathrm{~min}$. Then, the Grignard reagent (1.2-2.0 equiv.) was added dropwise at this temperature. The reaction was warmed up to $\mathrm{rt}$ and stirred until completion by TLC ( 1 to 3 hours generally). The mixture was then quenched with a saturated aq. solution $\mathrm{NH}_{4} \mathrm{Cl}$, extracted with EtOAc, washed with $\mathrm{H}_{2} \mathrm{O}$, dried over $\mathrm{MgSO}_{4}$ and concentrated under reduced pressure. $\mathrm{CH}_{2} \mathrm{Cl}_{2}$ was added to the solids obtained. The insoluble part was filtered and dried in vacuo to afford the desired product. If necessary, crude products were purified by column chromatography over silica gel (40 g $\mathrm{SiO}_{2}$, gradient, heptane/EtOAc, 100/0 to 50/50) to furnish the desired products. 


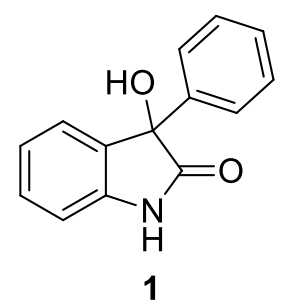

General procedure B was followed with isatin $(2.0 \mathrm{~g}, 13.6 \mathrm{mmol})$ and phenylmagnesium bromide (3.0 $\mathrm{M}$ in $\mathrm{Et}_{2} \mathrm{O}, 9.1 \mathrm{~mL}, 27.2 \mathrm{mmol}, 2.0$ equiv.). Purification was performed with flash column chromatography over silica gel (40 $\mathrm{g} \mathrm{SiO}_{2}$, heptane/EtOAc, 100/0 to 50/50, gradient) to afford 2a as a yellow solid (1.2 g, $5.33 \mathrm{mmol}, 39 \%) .{ }^{1} \mathbf{H}$ NMR (400 MHz, DMSO-d6): $\delta 10.40(\mathrm{~s}, 1 \mathrm{H}), 7.33-7.23(\mathrm{~m}, 6 \mathrm{H}), 7.10(\mathrm{~d}, J=6.9 \mathrm{~Hz}, 1 \mathrm{H}), 6.96(\mathrm{td}, J=7.7,1.1$ $\mathrm{Hz}, 1 \mathrm{H}), 6.91(\mathrm{~d}, J=7.7 \mathrm{~Hz}, 1 \mathrm{H}), 6.63(\mathrm{~s}, 1 \mathrm{H}) .{ }^{13} \mathrm{C}$ NMR (100 MHz, DMSO-d6): $\delta 178.5$ $(\mathrm{C}=\mathrm{O}), 142.0(\mathrm{C}), 141.6(\mathrm{C}), 133.8(\mathrm{C}), 129.2(\mathrm{CH}), 128.1(2 \mathrm{CH}), 127.4(\mathrm{CH}), 125.4(2 \mathrm{CH})$, $124.8(\mathrm{CH}), 122.1(\mathrm{CH}), 109.9(\mathrm{CH}), 77.3(\mathrm{C})$. FT-IR (neat, $\mathbf{~ c m}^{-1}$ ): 3408, 1703, 1615, 1467, 1360, 1338, 1180, 1156, 1119, 1067. HRMS (ESI $\left.{ }^{+}\right): m / z$ calcd. for $\mathrm{C}_{14} \mathrm{H}_{11} \mathrm{NO}_{2} \mathrm{Na}[\mathrm{M}+\mathrm{Na}]^{+}$ 248.0682, found 248.0670. The data are in agreement with those previously reported in the literature. ${ }^{1}$

\section{3-Hydroxy-3-(4-methoxyphenyl)indolin-2-one 3a}<smiles>COc1ccc(C2(O)C(=O)Nc3ccccc32)cc1</smiles>

$3 a$

General procedure $\mathbf{B}$ was followed with isatin $(0.50 \mathrm{~g}, 3.40 \mathrm{mmol})$ and 4-methoxyphenylmagnesium bromide ( $0.50 \mathrm{M}$ in THF, $9.5 \mathrm{~mL}, 4.76 \mathrm{mmol}, 1.4$ equiv.). Purification was performed with flash column chromatography over silica gel $\left(40 \mathrm{~g} \mathrm{SiO}_{2}\right.$, heptane/EtOAc, 100/0 to 50/50, gradient) to afford 3a as a white solid (0.65 mg, $2.55 \mathrm{mmol}$, 75\%). ${ }^{1}$ H NMR (400 MHz, DMSO-d6): $\delta 10.32$ (s, 1H), 7.24 (td, $\left.J=7.6,1.1 \mathrm{~Hz}, 1 \mathrm{H}\right), 7.18$ $(\mathrm{d}, J=8.8 \mathrm{~Hz}, 2 \mathrm{H}), 7.10(\mathrm{~d}, J=7.2 \mathrm{~Hz}, 1 \mathrm{H}), 6.96(\mathrm{t}, J=7.6 \mathrm{~Hz}, 1 \mathrm{H}), 6.89-6.85(\mathrm{~m}, 3 \mathrm{H}), 6.51$ (s, 1H), 3.71 (s, 3H). 13 C NMR (100 MHz, DMSO-d6): $\delta 178.6(\mathrm{C}=\mathrm{O}), 158.7$ (C), 141.9 (C), $133.7(\mathrm{C}), 133.5(\mathrm{C}), 129.1(\mathrm{CH}), 126.8(2 \mathrm{CH}), 124.8(\mathrm{CH}), 121.9(\mathrm{CH}), 113.4(2 \mathrm{CH}), 109.8$ (CH), $76.9(\mathrm{C}), 55.1\left(\mathrm{CH}_{3}\right)$. FT-IR (neat, $\left.\mathbf{c m}^{-1}\right)$ : 3270, 1718, 1684, 1610, 1509, 1466, 1347, 1297, 1250, 1177, 1123, 1106, 1076, 1033. HRMS (ESI $\left.{ }^{+}\right): \mathrm{m} / z$ calcd. for $\mathrm{C}_{15} \mathrm{H}_{13} \mathrm{NO}_{3} \mathrm{Na}$ $[\mathrm{M}+\mathrm{Na}]^{+}$278.0788, found 278.0775. The data are in agreement with those previously reported in the literature. ${ }^{2}$ 


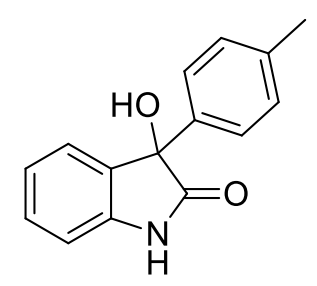

3b

General procedure $\mathbf{B}$ was followed with with isatin $(0.50 \mathrm{~g}, 3.40 \mathrm{mmol})$ and p-tolylmagnesium bromide prepared from 4-bromotoluene $(0.75 \mathrm{~mL}, 6.12 \mathrm{mmol}, 1.8$ equiv.). Purification was performed with flash column chromatography over silica gel $\left(40 \mathrm{~g} \mathrm{SiO}_{2}\right.$, heptane/EtOAc, $100 / 0$ to 50/50, gradient) to afford $\mathbf{3 b}$ as an off white solid $(0.20 \mathrm{~g}$, 0.83 mmol, 24\%). ${ }^{1} \mathbf{H}$ NMR (600 MHz, CDCl 3$): \delta 8.87$ (brs, $\left.1 \mathrm{H}\right), 7.24$ (t, $\left.J=7.8 \mathrm{~Hz}, 1 \mathrm{H}\right)$, $7.16(\mathrm{~d}, J=7.9 \mathrm{~Hz}, 2 \mathrm{H}), 7.13-7.10(\mathrm{~m}, 3 \mathrm{H}), 7.02(\mathrm{t}, J=7.5 \mathrm{~Hz}, 1 \mathrm{H}), 6.93(\mathrm{~d}, J=7.8 \mathrm{~Hz}, 1 \mathrm{H})$, 4.60 (s, 1H), 2.34 (s, 3H). ${ }^{13}$ C NMR (150 MHz, CDCl3): $\delta 179.1(\mathrm{C}=\mathrm{O}), 141.8$ (C), 137.5 (C), $133.6(\mathrm{C}), 130.0(\mathrm{C}), 129.8(2 \mathrm{CH}), 128.5(2 \mathrm{CH}), 128.4(\mathrm{CH}), 125.4(\mathrm{CH}), 122.8(\mathrm{CH})$, $110.1(\mathrm{CH}), 52.5(\mathrm{CH}), 21.3\left(\mathrm{CH}_{3}\right)$. HRMS $\left(\mathbf{E S I}^{+}\right): \mathrm{m} / z$ calcd. for $\mathrm{C}_{15} \mathrm{H}_{13} \mathrm{NO}_{2} \mathrm{Na}[\mathrm{M}+\mathrm{Na}]^{+}$ 262.0838, found 262.0839. The data are in agreement with those previously reported in the literature. $^{2}$

3-(4-(Tert-butyl)phenyl)-3-hydroxyindolin-2-one 3c<smiles>CC(C)(C)c1ccc(C2(O)C(=O)Nc3ccccc32)cc1</smiles>

General procedure B was followed with isatin $(0.50 \mathrm{~g}, 3.40 \mathrm{mmol})$ and (4-(tertbutyl)phenyl)magnesium bromide prepared from 1-bromo-4-tert-butylbenzene $(1.1 \mathrm{~mL}$, $6.12 \mathrm{mmol}, 1.8$ equiv.). Purification was performed with flash column chromatography over silica gel (40 $\mathrm{g} \mathrm{SiO}_{2}$, heptane/EtOAc, 100/0 to 50/50, gradient) to afford $\mathbf{3 c}$ as a yellow solid (0.59 g, 2.08 mmol, 61\%). ${ }^{1}$ H NMR (600 MHz, DMSO-d6): $\delta 10.36$ (s, 1H), 7.32 (d, $J=8.5$ $\mathrm{Hz}, 2 \mathrm{H}), 7.24(\mathrm{td}, J=7.7,0.8 \mathrm{~Hz}, 1 \mathrm{H}), 7.19(\mathrm{~d}, J=8.5 \mathrm{~Hz}, 2 \mathrm{H}), 7.10(\mathrm{~d}, J=7.4 \mathrm{~Hz}, 1 \mathrm{H}), 6.96$ (t, $J=7.4 \mathrm{~Hz}, 1 \mathrm{H}), 6.88(\mathrm{~d}, J=7.7 \mathrm{~Hz}, 1 \mathrm{H}), 6.53(\mathrm{~s}, 1 \mathrm{H}), 1.24$ (s, 9H). ${ }^{13} \mathbf{C}$ NMR (150 MHz, DMSO-d6): $\delta 178.6(\mathrm{C}=\mathrm{O}), 149.8(\mathrm{C}), 141.9(\mathrm{C}), 138.6(\mathrm{C}), 133.8(\mathrm{C}), 129.2(\mathrm{CH}), 125.2$ $(2 \mathrm{CH}), 124.9(2 \mathrm{CH}), 124.8(\mathrm{CH}), 122.0(\mathrm{CH}), 109.8(\mathrm{CH}), 77.2(\mathrm{C}), 34.2(\mathrm{C}), 31.1\left(3 \mathrm{CH}_{3}\right)$. HRMS (ESI ${ }^{+}$): $m / z$ calcd. for $\mathrm{C}_{18} \mathrm{H}_{19} \mathrm{NO}_{2} \mathrm{Na}[\mathrm{M}+\mathrm{Na}]^{+} 304.1308$, found 304.1306. The data are in agreement with those previously reported in the literature. ${ }^{3}$ 


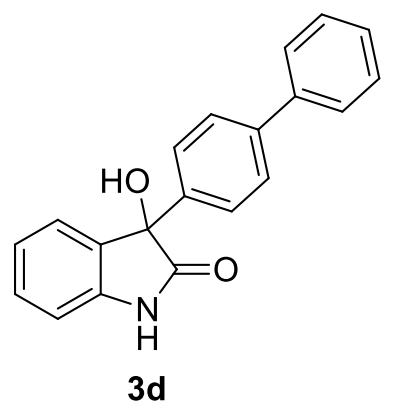

General procedure $\mathbf{B}$ was followed with isatin (1.0 g, $6.80 \mathrm{mmol})$ and [1,1'-biphenyl]-4ylmagnesium bromide prepared from 4-bromobiphenyl (3.4 mL, $13.6 \mathrm{mmol}, 2.0$ equiv.). Purification was performed with flash column chromatography over silica gel $\left(40 \mathrm{~g} \mathrm{SiO}_{2}\right.$, heptane/EtOAc, 100/0 to 50/50, gradient) to afford 3d as a white solid (1.1 g, $3.54 \mathrm{mmol}$, 52\%). ${ }^{1}$ H NMR (600 MHz, DMSO-d6): $\delta 10.43$ (s, 1H), 7.67-7.57 (m, 4H), 7.50-7.40 (m, 2H), 7.40-7.31 (m, 3H), 7.31-7.22 (m, 1H), 7.14 (d, J=6.7 Hz, 1H), 7.03-6.87 (m, 2H), 6.68 (s, 1H). ${ }^{13}$ C NMR (150 MHz, DMSO-d6): $\delta 178.4$ (C=O), 142.0 (C), 140.7 (C), 139.9 (C), $139.4(\mathrm{C}), 133.7(\mathrm{C}), 129.3(\mathrm{CH}), 128.9(2 \mathrm{CH}), 127.5(\mathrm{CH}), 126.7(2 \mathrm{CH}), 126.5(2 \mathrm{CH}), 126.1$ $(2 \mathrm{CH}), 124.8(\mathrm{CH}), 122.1(\mathrm{CH}), 109.9(\mathrm{CH}), 77.2(\mathrm{C}) . \mathbf{H R M S}\left(\mathbf{E S I}^{+}\right): \mathrm{m} / \mathrm{z}$ calcd. for $\mathrm{C}_{20} \mathrm{H}_{15} \mathrm{NO}_{2} \mathrm{Na}[\mathrm{M}+\mathrm{Na}]^{+} 324.0995$, found 324.0997. The data are in agreement with those previously reported in the literature. ${ }^{3}$

\section{3-(4-Chlorophenyl)-3-hydroxyindolin-2-one 3e}<smiles>O=C1Nc2ccccc2C1(O)c1ccc(Cl)cc1</smiles>

General procedure $\mathbf{B}$ was followed with isatin $(0.50 \mathrm{~g}, 3.40 \mathrm{mmol})$ and chlorophenylmagnesium bromide (1.0 M in THF, $6.8 \mathrm{~mL}, 6.80 \mathrm{mmol}, 2.0$ equiv.). Purification was performed with flash column chromatography over silica gel $\left(40 \mathrm{~g} \mathrm{SiO}_{2}\right.$, heptane/EtOAc, 100/0 to 50/50, gradient) to afford $\mathbf{3 e}$ as a beige solid (0.20 g, $0.77 \mathrm{mmol}, 23 \%) .{ }^{1} \mathbf{H}$ NMR (600 MHz, DMSO-d6): $\delta 10.45$ (s, 1H), 7.32 (d, $J=8.5 \mathrm{~Hz}, 2 \mathrm{H}), 7.28-7.24$ (m, 3H), 7.10 (d, $J=7.3 \mathrm{~Hz}, 1 \mathrm{H}), 6.98(\mathrm{t}, J=7.5 \mathrm{~Hz}, 1 \mathrm{H}), 6.91(\mathrm{~d}, J=7.8 \mathrm{~Hz}, 1 \mathrm{H}), 6.74(\mathrm{~s}, 1 \mathrm{H}) .{ }^{13} \mathbf{C ~ N M R}$ (150 MHz, DMSO-d6): $\delta 178.1(\mathrm{C}=\mathrm{O}), 142.0$ (C), 140.5 (C), 133.2 (C), 132.2 (C), 129.5 $(\mathrm{CH}), 128.2(2 \mathrm{CH}), 127.4(2 \mathrm{CH}), 124.8(\mathrm{CH}), 122.2(\mathrm{CH}), 110.0(\mathrm{CH}), 76.9(\mathrm{C})$. FT-IR (neat, $\mathbf{~ c m}^{-1}$ ): $3189,1714,1620,1489,1471,1396,1181,1102$. HRMS (ESI $\left.{ }^{+}\right): ~ m / z$ calcd. for $\mathrm{C}_{14} \mathrm{H}_{10} \mathrm{ClNO}_{2} \mathrm{Na}[\mathrm{M}+\mathrm{Na}]^{+}$282.0292, found 282.0293. 


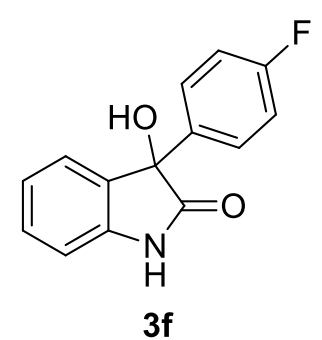

General procedure $\mathbf{B}$ was followed with isatin $(0.50 \mathrm{~g}, 3.40 \mathrm{mmol})$ and fluorophenylmagnesium bromide (1.0 M in THF, $6.1 \mathrm{~mL}, 6.12 \mathrm{mmol}, 1.8$ equiv.). Purification was performed with flash column chromatography over silica gel (40 g $\mathrm{SiO}_{2}$, heptane/EtOAc, $100 / 0$ to $50 / 50$, gradient) to afford $\mathbf{3 f}$ as a light yellow solid $(0.45 \mathrm{~g}, 1.86 \mathrm{mmol}, 55 \%) .{ }^{\mathbf{1}} \mathbf{H}$ NMR (600 MHz, DMSO-d6): $\delta 10.42(\mathrm{~s}, 1 \mathrm{H}), 7.32-7.27(\mathrm{~m}, 2 \mathrm{H}), 7.26(\mathrm{td}, J=7.7,1.2 \mathrm{~Hz}$, 1H), 7.17-7.09 (m, 3H), 6.98 (td, $J=7.5,0.7 \mathrm{~Hz}, 1 \mathrm{H}), 6.90$ (d, $J=7.7 \mathrm{~Hz}, 1 \mathrm{H}), 6.69$ (s, 1H). ${ }^{13}$ C NMR (150 MHz, DMSO-d6): $\delta 178.3(\mathrm{C}=\mathrm{O}), 161.6\left(\mathrm{~d}, J^{\mathrm{CF}}=243.5 \mathrm{~Hz}, \mathrm{C}\right), 141.9(\mathrm{C})$, $137.7\left(\mathrm{~d}, J^{\mathrm{CF}}=2.8 \mathrm{~Hz}, \mathrm{C}\right), 133.4(\mathrm{C}), 129.4(\mathrm{CH}), 127.6\left(\mathrm{~d}, J^{\mathrm{CF}}=8.3 \mathrm{~Hz}, 2 \mathrm{CH}\right), 124.8(\mathrm{CH})$, $122.2(\mathrm{CH}), 114.9\left(\mathrm{~d}, J^{\mathrm{CF}}=21.4 \mathrm{~Hz}, 2 \mathrm{CH}\right), 110.0(\mathrm{CH}), 76.8(\mathrm{C}) .{ }^{19} \mathbf{F}$ NMR (565 MHz, DMSO-d6): $\delta$-115.2. FT-IR (neat, $\mathbf{c m}^{-1}$ ): 3189, 1714, 1620, 1489, 1471, 1396, 1181, 1102. HRMS (ESI ${ }^{+}$): $m / z$ calcd. for $\mathrm{C}_{14} \mathrm{H}_{10} \mathrm{FNO}_{2} \mathrm{Na}[\mathrm{M}+\mathrm{Na}]^{+}$266.0588, found 266.0591. All analytical data were in accordance with literature. ${ }^{3}$

\section{3-Hydroxy-3-(3-methoxyphenyl)indolin-2-one 3g}

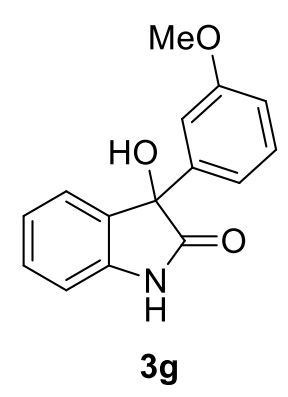

General procedure $\mathbf{B}$ was followed with isatin $(0.50 \mathrm{~g}, 3.40 \mathrm{mmol})$ and 3-methoxyphenylmagnesium bromide (1.0 M in 2-MeTHF, $4.8 \mathrm{~mL}, 4.76 \mathrm{mmol}, 1.4$ equiv.). Purification was performed with flash column chromatography over silica gel (40 g $\mathrm{SiO}_{2}$, heptane/EtOAc, 100/0 to 50/50, gradient) to afford $\mathbf{3 g}$ as a white solid $(0.2 \mathrm{~g}, 0.78 \mathrm{mmol}$, 23\%). ${ }^{1}$ H NMR (400 MHz, DMSO-d6): $\delta 10.39$ (s, $\left.1 \mathrm{H}\right), 7.24$ (td, $\left.J=7.7,1.1 \mathrm{~Hz}, 1 \mathrm{H}\right), 7.19$ (t, $J=8.0 \mathrm{~Hz}, 1 \mathrm{H}), 7.09(\mathrm{~d}, J=7.2 \mathrm{~Hz}, 1 \mathrm{H}), 6.96(\mathrm{~m}, 2 \mathrm{H}), 6.89(\mathrm{~d}, J=7.7 \mathrm{~Hz}, 1 \mathrm{H}), 6.83$ (dd, $J=8.0,2.2 \mathrm{~Hz}, 1 \mathrm{H}), 6.69(\mathrm{~d}, J=7.8 \mathrm{~Hz}, 1 \mathrm{H}), 6.62(\mathrm{~s}, 1 \mathrm{H}), 3.72(\mathrm{~s}, 3 \mathrm{H}) .{ }^{13} \mathbf{C}$ NMR (100 MHz, DMSO-d6): $\delta 178.3$ (C=O), 159.1 (C), 143.1 (C), 141.9 (C), 133.7 (C), 129.2 $(\mathrm{CH}), 129.2(\mathrm{CH}), 124.7(\mathrm{CH}), 122.0(\mathrm{CH}), 117.5(\mathrm{CH}), 112.5(\mathrm{CH}), 111.5(\mathrm{CH}), 109.8(\mathrm{CH})$, $77.2(\mathrm{C}), 55.0\left(\mathrm{CH}_{3}\right)$. FT-IR (neat, $\left.\mathbf{c m}^{-1}\right)$ : 3405, 1703, 1615, 1607, 1585, 1485, 1467, 1433, 1338, 1285, 1245, 1179, 1149, 1119, 1098, 1076, 1044. HRMS (ESI $\left.{ }^{+}\right): m / z$ calcd. for $\mathrm{C}_{15} \mathrm{H}_{13} \mathrm{NO}_{3} \mathrm{Na}[\mathrm{M}+\mathrm{Na}]^{+} 278.0788$, found 278.0804 . The data are in agreement with those previously reported in the literature. ${ }^{4}$ 
<smiles>O=C1Nc2ccccc2C1(O)c1c(F)c(F)c(F)c(F)c1F</smiles>

General procedure $\mathbf{B}$ was followed with isatin $(0.50 \mathrm{~g}, 2.04 \mathrm{mmol})$ and (perfluorophenyl)magnesium bromide prepared from bromopentafluorobenzene $(1.2 \mathrm{M}$ in $\mathrm{Et}_{2} \mathrm{O}, 6.0 \mathrm{~mL}, 6.12 \mathrm{mmol}, 1.8$ equiv.). Purification was performed with flash column chromatography over silica gel (40 $\mathrm{g} \mathrm{SiO}_{2}$, heptane/EtOAc, 100/0 to 50/50, gradient) to afford 3h as a yellow solid (0.95 g, $3.01 \mathrm{mmol}, 89 \%) .{ }^{1} \mathbf{H}$ NMR (700 MHz, DMSO-d6): $\delta 10.67$ (brs, 1H), $7.27(\mathrm{td}, J=7.7,1.2 \mathrm{~Hz}, 1 \mathrm{H}), 7.24(\mathrm{~d}, J=7.4 \mathrm{~Hz}, 1 \mathrm{H}), 7.17$ (s, 1H), 6.97 (td, $J=$ 7.6, $0.9 \mathrm{~Hz}, 1 \mathrm{H}), 6.90$ (d, J = 7.7 Hz, 1H). ${ }^{\mathbf{1 3}} \mathbf{C}$ NMR (176 MHz, DMSO-d6): $\delta 175.6(\mathrm{C}=\mathrm{O})$, $144.5(\mathrm{dm}, J=251.6 \mathrm{~Hz}, 2 \mathrm{C}), 141.8(\mathrm{C}), 139.9(\mathrm{dm}, J=252.0 \mathrm{~Hz}, \mathrm{C}), 137.3(\mathrm{dm}, J=248.3$ $\mathrm{Hz}, 2 \mathrm{C}), 130.4(\mathrm{CH}), 130.4(\mathrm{C}), 124.8(\mathrm{CH}), 122.2(\mathrm{CH}), 115.6(\mathrm{~m}, \mathrm{C}), 110.2(\mathrm{CH}), 75.2(\mathrm{C})$. ${ }^{19}$ F NMR (659 MHz, DMSO-d6): $\delta 139.5(\mathrm{~d}, J=19.9 \mathrm{~Hz}),-155.2(\mathrm{t}, J=22.4 \mathrm{~Hz}),-162.5$ $(\mathrm{dd}, J=22.4,19.9 \mathrm{~Hz})$. HRMS $\left(\mathbf{E S I}^{+}\right): \mathrm{m} / z$ calcd. for $\mathrm{C}_{14} \mathrm{H}_{6} \mathrm{~F}_{5} \mathrm{NO}_{2} \mathrm{Na}[\mathrm{M}+\mathrm{Na}]^{+} 338.0211$, found 338.0208.

\section{3-Hydroxy-3-methylindolin-2-one 3i}<smiles>CC1(O)C(=O)Nc2ccccc21</smiles>

3i

General procedure B was followed with isatin $(0.50 \mathrm{~g}, 3.40 \mathrm{mmol})$ and methylmagnesium bromide ( $3.0 \mathrm{M}$ in $\mathrm{Et}_{2} \mathrm{O}, 2.3 \mathrm{~mL}, 6.80 \mathrm{mmol}, 2.0$ equiv.). Purification was performed with flash column chromatography over silica gel (40 $\mathrm{g} \mathrm{SiO}_{2}$, heptane/EtOAc, 100/0 to 50/50, gradient) to afford 3i as a light yellow solid (0.39 g, $2.39 \mathrm{mmol}, 70 \%) .{ }^{\mathbf{1}} \mathbf{H}$ NMR (600 $\mathbf{~ M H z}$, DMSO-d6): 10.19 (s, 1H), 7.28 (d, $J=7.3 \mathrm{~Hz}, 1 \mathrm{H}), 7.18$ (t, $J=7.6 \mathrm{~Hz}, 1 \mathrm{H}), 6.96$ (t, $J=7.4$ $\mathrm{Hz}, 1 \mathrm{H}), 6.80(\mathrm{~d}, J=7.7 \mathrm{~Hz}, 1 \mathrm{H}), 5.84(\mathrm{~s}, 1 \mathrm{H}), 1.35(\mathrm{~s}, 3 \mathrm{H}) .{ }^{13} \mathbf{C}$ NMR (150 MHz, DMSO-d6): $\delta 179.7(\mathrm{C}=\mathrm{O}), 141.1(\mathrm{C}), 133.6(\mathrm{C}), 128.8(\mathrm{CH}), 123.4(\mathrm{CH}), 121.6(\mathrm{CH}), 109.6$ $(\mathrm{CH}), 72.6(\mathrm{C}), 24.5\left(\mathrm{CH}_{3}\right)$. HRMS (ESI $\left.{ }^{+}\right): \mathrm{m} / z$ calcd. for $\mathrm{C}_{9} \mathrm{H}_{9} \mathrm{NO}_{2} \mathrm{Na}[\mathrm{M}+\mathrm{Na}]^{+}$186.0525, found 186.0524 . The data are in agreement with those previously reported in the literature. ${ }^{5}$

\section{3-Hydroxy-3-isopropylindolin-2-one 3j}<smiles>CC(C)C1(O)C(=O)Nc2ccccc21</smiles> 
General procedure $\mathbf{B}$ was followed with isatin $(0.50 \mathrm{~g}, 3.40 \mathrm{mmol})$ and isopropylmagnesium chloride ( $2.0 \mathrm{M}$ in THF, $2.4 \mathrm{~mL}, 4.76 \mathrm{mmol}, 1.4$ equiv.). Purification was performed with flash column chromatography over silica gel (40 $\mathrm{g} \mathrm{SiO}_{2}$, heptane/EtOAc, 100/0 to 50/50, gradient) to afford $\mathbf{3 j}$ as a light yellow solid (70 $\mathrm{mg}, 0.37 \mathrm{mmol}, 11 \%) .{ }^{\mathbf{1}} \mathbf{H}$ NMR (400 $\mathbf{~ M H z}$, DMSO-d6): $\delta 10.19$ (s, 1H), 7.23-7.17 (m, 2H), 6.94 (td, $J=7.6,0.8 \mathrm{~Hz}, 1 \mathrm{H}), 6.79$ (d, $J=7.6$ $\mathrm{Hz}, 1 \mathrm{H}), 5.76(\mathrm{~s}, 1 \mathrm{H}), 2.11-2.01(\mathrm{~m}, 1 \mathrm{H}), 0.96(\mathrm{~d}, J=6.9 \mathrm{~Hz}, 3 \mathrm{H}), 0.63(\mathrm{~d}, J=6.9 \mathrm{~Hz}, 3 \mathrm{H})$. ${ }^{13}$ C NMR (100 MHz, DMSO-d6): $\delta 179.5(\mathrm{C}=\mathrm{O}), 142.2(\mathrm{C}), 130.6(\mathrm{C}), 128.7(\mathrm{CH}), 124.6$ $(\mathrm{CH}), 121.3(\mathrm{CH}), 109.3(\mathrm{CH}), 78.3(\mathrm{C}), 34.8(\mathrm{CH}), 16.2\left(\mathrm{CH}_{3}\right), 15.8\left(\mathrm{CH}_{3}\right)$. FT-IR (neat, $\left.\mathbf{~ c m}^{-1}\right)$ : 3346, 1699, 1621, 1469, 1357, 1194, 1179, 1123, 1095, 1076. HRMS (ESI'): $m / z$ calcd. for $\mathrm{C}_{11} \mathrm{H}_{13} \mathrm{NO}_{2} \mathrm{Na}[\mathrm{M}+\mathrm{Na}]^{+} 214.0838$, found 214.0853 . The data are in agreement with those previously reported in the literature. ${ }^{6}$

\section{3-Cyclopentyl-3-hydroxyindolin-2-one 3k}

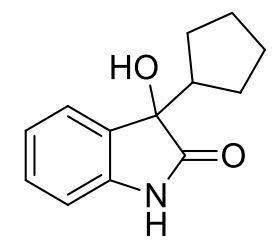

3k

General procedure $\mathbf{B}$ was followed with isatin $(0.30 \mathrm{~g}, 2.04 \mathrm{mmol})$ and cyclopentylmagnesium bromide (2.0 M in THF, $1.4 \mathrm{~mL}, 2.85 \mathrm{mmol}, 1.4$ equiv.). Purification was performed with flash column chromatography over silica gel (40 $\mathrm{g} \mathrm{SiO}_{2}$, heptane/EtOAc, 100/0 to 50/50, gradient) to afford $\mathbf{3 k}$ as a light orange solid $(0.15 \mathrm{~g}, 0.69 \mathrm{mmol}, 34 \%) .{ }^{\mathbf{1}} \mathbf{H}$ NMR (500 MHz, CDCl $): \delta 8.01$ (brs, $1 \mathrm{H}), 7.40(\mathrm{~d}, J=7.3 \mathrm{~Hz}, 1 \mathrm{H}), 7.25(\mathrm{td}, J=7.7,1.2 \mathrm{~Hz}$, $1 \mathrm{H}), 7.05(\mathrm{t}, J=7.3 \mathrm{~Hz}, 1 \mathrm{H}), 6.87(\mathrm{~d}, J=7.7 \mathrm{~Hz}, 1 \mathrm{H}), 2.51-2.41(\mathrm{~m}, 1 \mathrm{H}), 1.82-1.72(\mathrm{~m}, 1 \mathrm{H})$, 1.72-1.60 (m, 2H), 1.58-1.45 (m, 4H), 1.32-1.21 (m, 1H). ${ }^{13}$ C NMR (126 MHz, CDCl $): \delta$ $180.7(\mathrm{C}=\mathrm{O}), 140.7(\mathrm{C}), 130.2(\mathrm{C}), 129.6(\mathrm{CH}), 125.1(\mathrm{CH}), 123.0(\mathrm{CH}), 110.2(\mathrm{CH}), 78.6$ (C), $47.7(\mathrm{CH}), 26.6\left(\mathrm{CH}_{2}\right), 26.5\left(\mathrm{CH}_{2}\right), 25.7\left(\mathrm{CH}_{2}\right), 25.5\left(\mathrm{CH}_{2}\right)$. HRMS (ESI $\left.{ }^{+}\right): \mathrm{m} / \mathrm{z}$ calcd. for $\mathrm{C}_{13} \mathrm{H}_{15} \mathrm{NO}_{2} \mathrm{Na}[\mathrm{M}+\mathrm{Na}]^{+} 240.0995$, found 240.0996 .

\section{3-Allyl-3-hydroxyindolin-2-one $3 l$}

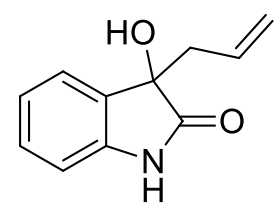

3I

General procedure $\mathbf{B}$ was followed with isatin $(0.50 \mathrm{~g}, 3.40 \mathrm{mmol})$ and allylmagnesium bromide (1.0 M in $\mathrm{Et}_{2} \mathrm{O}, 6.8 \mathrm{~mL}, 6.80 \mathrm{mmol}, 2.0$ equiv.). Purification was performed with flash column chromatography over silica gel (40 $\mathrm{g} \mathrm{SiO}_{2}$, heptane/EtOAc, 100/0 to 50/50, gradient) to afford 3k as a light yellow solid (0.37 g, $1.94 \mathrm{mmol}, 57 \%) .{ }^{\mathbf{1}} \mathbf{H}$ NMR (400 MHz, CDCl3): $\delta 8.33$ (brs, $1 \mathrm{H}), 7.37(\mathrm{~d}, J=7.5 \mathrm{~Hz}, 1 \mathrm{H}), 7.26(\mathrm{dt}, J=8.8,6.5 \mathrm{~Hz}, 1 \mathrm{H}), 7.07(\mathrm{t}, J=$ $7.5 \mathrm{~Hz}, 1 \mathrm{H}), 6.88(\mathrm{~d}, J=7.7 \mathrm{~Hz}, 1 \mathrm{H}), 5.73-5.61(\mathrm{~m}, 1 \mathrm{H}), 5.15-5.08(\mathrm{~m}, 2 \mathrm{H}), 3.36(\mathrm{~s}, 1 \mathrm{H})$, $2.75(\mathrm{dd}, J=13.4,6.4 \mathrm{~Hz}, 1 \mathrm{H}), 2.62(\mathrm{dd}, J=13.4,8.2 \mathrm{~Hz}, 1 \mathrm{H}) .{ }^{13} \mathbf{C}$ NMR (100 MHz, CDCl3): $\delta 180.3(\mathrm{C}=\mathrm{O}), 140.4(\mathrm{C}), 130.4(\mathrm{CH}), 130.3(\mathrm{C}), 129.8(\mathrm{CH}), 124.6(\mathrm{CH}), 123.2$ $(\mathrm{CH}), 120.7\left(\mathrm{CH}_{2}\right), 110.5(\mathrm{CH}), 76.4(\mathrm{C}), 43.0\left(\mathrm{CH}_{2}\right)$. FT-IR (neat, $\left.\mathbf{c m}^{-1}\right)$ : 3322, 3221, 3064, 
2714, 1681, 1624, 1472, 1355, 1274, 1188, 1110, 1091. HRMS (ESI $\left.{ }^{+}\right): m / z$ calcd. for $\mathrm{C}_{11} \mathrm{H}_{11} \mathrm{NO}_{2} \mathrm{Na}[\mathrm{M}+\mathrm{Na}]^{+}$212.0682, found 212.0675.

\section{3-Hydroxy-5-methyl-3-phenylindolin-2-one 5a}<smiles>Cc1ccc2c(c1)C(O)(c1ccccc1)C(=O)N2</smiles>

General procedure B was followed with 5-methylisatin $(0.50 \mathrm{~g}, 3.10 \mathrm{mmol})$ and phenylmagnesium bromide ( $3.0 \mathrm{M}$ in $\mathrm{Et}_{2} \mathrm{O}, 2.1 \mathrm{~mL}, 6.21 \mathrm{mmol}, 2.0$ equiv.). Purification was performed with flash column chromatography over silica gel $\left(40 \mathrm{~g} \mathrm{SiO}_{2}\right.$, heptane/EtOAc, 100/0 to 50/50, gradient) to afford $\mathbf{5 a}$ as a white solid (0.46 g, $1.91 \mathrm{mmol}, 61 \%) .{ }^{1} \mathbf{H}$ NMR (400 MHz, DMSO-d6): $\delta 10.30$ (s, 1H), 7.33-7.23 (m, 5H), 7.04 (d, J = 7.8 Hz, 1H), 6.90 (s, 1H), 6.79 (d, $J=7.8 \mathrm{~Hz}, 1 \mathrm{H}), 6.57(\mathrm{~s}, 1 \mathrm{H}), 2.21$ (s, 3H). ${ }^{13}$ C NMR (100 MHz, DMSO-d6): $\delta$ $178.5(\mathrm{C}=\mathrm{O}), 141.7(\mathrm{C}), 139.4(\mathrm{C}), 133.9(\mathrm{C}), 130.9(\mathrm{C}), 129.4(\mathrm{CH}), 128.1(2 \mathrm{CH}), 127.3$ $(\mathrm{CH}), 125.4(2 \mathrm{CH}), 125.3(\mathrm{CH}), 109.6(\mathrm{CH}), 77.4(\mathrm{C}), 20.60\left(\mathrm{CH}_{3}\right)$. FT-IR (neat, $\left.\mathbf{c m}^{-1}\right)$ : 3209, 1697, 1624, 1491, 1202, 1190, 1149, 1139, 1128. HRMS (ESI $\left.{ }^{+}\right): m / z$ calcd. for $\mathrm{C}_{15} \mathrm{H}_{13} \mathrm{NO}_{2} \mathrm{Na}[\mathrm{M}+\mathrm{Na}]^{+} 262.0838$, found 262.0853. The data are in agreement with those previously reported in the literature. $^{2}$

\section{5-Bromo-3-hydroxy-3-phenylindolin-2-one 5b}<smiles>O=C1Nc2ccc(Br)cc2C1(O)c1ccccc1</smiles>

$5 b$

General procedure $\mathbf{B}$ was followed with 5-bromoisatin $(0.32 \mathrm{~g}, 1.39 \mathrm{mmol})$ and phenylmagnesium bromide ( $3.0 \mathrm{M}_{\text {in }} \mathrm{Et}_{2} \mathrm{O}, 0.93 \mathrm{~mL}, 2.79 \mathrm{mmol}, 2.0$ equiv.). Purification was performed with flash column chromatography over silica gel (40 g $\mathrm{SiO}_{2}$, heptane/EtOAc, 100/0 to 50/50, gradient) to afford $\mathbf{5 b}$ as a light yellow solid (0.30 mg, $0.98 \mathrm{mmol}, 71 \%) .{ }^{\mathbf{1}} \mathbf{H}$ NMR (600 MHz, DMSO-d6): $\delta 10.57$ (brs, 1H), 7.44 (dd, $J=8.3,2.1 \mathrm{~Hz}, 1 \mathrm{H}), 7.36-7.31$ (m, 2H), 7.30-7.26 (m, 3H), $7.20(\mathrm{~d}, J=2.1 \mathrm{~Hz}, 1 \mathrm{H}), 6.88(\mathrm{~d}, J=8.3 \mathrm{~Hz}, 1 \mathrm{H}), 6.79(\mathrm{~s}, 1 \mathrm{H})$. ${ }^{13}$ C NMR (150 MHz, DMSO-d6): $\delta 178.0(\mathrm{C}=\mathrm{O}), 141.3$ (C), 140.8 (C), 136.2 (C), 132.0 $(\mathrm{CH}), 128.3(2 \mathrm{CH}), 127.7(\mathrm{CH}), 127.4(\mathrm{CH}), 125.3(2 \mathrm{CH}), 113.7(\mathrm{C}), 112.1(\mathrm{CH}), 77.4(\mathrm{C})$. HRMS (ESI ${ }^{+}$): $m / z$ calcd. for $\mathrm{C}_{14} \mathrm{H}_{10} \mathrm{BrNO}_{2} \mathrm{Na}[\mathrm{M}+\mathrm{Na}]^{+} 325.9787$, found 325.9786 . The data are in agreement with those previously reported in the literature. ${ }^{3}$ 
<smiles>COc1ccc2c(c1)NC(=O)C2(O)c1ccccc1</smiles>

$5 d$

General procedure $\mathbf{B}$ was followed with 6-methoxylisatin $(0.30 \mathrm{~g}, 1.69 \mathrm{mmol})$ and phenylmagnesium bromide ( $3.0 \mathrm{M}^{\text {in }} \mathrm{Et}_{2} \mathrm{O}, 0.84 \mathrm{~mL}, 1.49 \mathrm{mmol}, 1.5$ equiv.). Purification was performed with flash column chromatography over silica gel (40 g $\mathrm{SiO}_{2}$, heptane/EtOAc, 100/0 to 50/50, gradient) to afford $\mathbf{5 d}$ as a light yellow solid $(0.13 \mathrm{~g}, 0.51 \mathrm{mmol}, 30 \%) .{ }^{\mathbf{1}} \mathbf{H}$ NMR (600 MHz, DMSO) $\delta 10.34$ (brs, 1H), 7.32-7.28 (m, 2H), 7.28-7.22 (m, 3H), 6.99 (d, $J$ $=8.2 \mathrm{~Hz}, 1 \mathrm{H}), 6.51(\mathrm{dd}, J=8.2,2.3 \mathrm{~Hz}, 1 \mathrm{H}), 6.49(\mathrm{brs}, 1 \mathrm{H}), 6.45(\mathrm{~d}, J=2.3 \mathrm{~Hz}, 1 \mathrm{H}), 3.75(\mathrm{~s}$, 3H). ${ }^{13}$ C NMR (150 MHz, DMSO) $\delta 178.9$ (C=O), 160.3 (C), 143.3 (C), 141.9 (C), 128.0 $(2 \mathrm{CH}), 127.3(\mathrm{CH}), 125.7(\mathrm{CH}), 125.7(\mathrm{C}), 125.5(2 \mathrm{CH}), 106.8(\mathrm{CH}), 96.6(\mathrm{CH}), 77.0(\mathrm{C})$, $55.3\left(\mathrm{CH}_{3}\right)$. HRMS (ESI $\left.{ }^{+}\right): \mathrm{m} / z$ calcd. for $\mathrm{C}_{15} \mathrm{H}_{13} \mathrm{NO}_{3} \mathrm{Na}[\mathrm{M}+\mathrm{Na}]^{+} 278.0788$, found 278.0790 . The data are in agreement with those previously reported in the literature. ${ }^{7}$

\section{3-Hydroxy-7-methyl-3-phenylindolin-2-one 5e}<smiles>Cc1cccc2c1NC(=O)C2(O)c1ccccc1</smiles>

General procedure B was followed with 7-methylisatin $(0.20 \mathrm{~g}, 1.24 \mathrm{mmol})$ and phenylmagnesium bromide ( $3.0 \mathrm{M}_{\text {in }} \mathrm{Et}_{2} \mathrm{O}, 0.50 \mathrm{~mL}, 1.49 \mathrm{mmol}, 1.2$ equiv.). Purification was performed with flash column chromatography over silica gel (40 g $\mathrm{SiO}_{2}$, heptane/EtOAc, 100/0 to 50/50, gradient) to afford 5e as a light yellow solid (0.12 g, $0.54 \mathrm{mmol}, 44 \%) .{ }^{\mathbf{1}} \mathbf{H}$ NMR (400 MHz, DMSO-d6): $\delta 10.43$ (s, 1H), 7.33-7.22 (m 5H), 7.07 (d, J=7.0 Hz, 1H), 6.93-6.84 (m, 2H), 6.57 (s, 1H), 2.25 (s, 3H). ${ }^{13}$ C NMR (100 MHz, DMSO-d6): $\delta 178.9$ $(\mathrm{C}=\mathrm{O}), 141.7(\mathrm{C}), 140.4(\mathrm{C}), 133.4(\mathrm{C}), 130.4(\mathrm{CH}), 128.0(2 \mathrm{CH}), 127.3(\mathrm{CH}), 125.4(2 \mathrm{CH})$, $122.1(\mathrm{CH}), 122.0(\mathrm{CH}), 119.2(\mathrm{C}), 77.5(\mathrm{C}), 16.4\left(\mathrm{CH}_{3}\right)$. FT-IR (neat, $\left.\mathbf{c m}^{-1}\right)$ : 3165, 1720, 1626, 1606, 1486, 1448, 1381, 1300, 1209, 1191, 1163, 1118, 1102, 1070. HRMS (ESI'): $m / z$ calcd. for $\mathrm{C}_{15} \mathrm{H}_{13} \mathrm{NO}_{2} \mathrm{Na}[\mathrm{M}+\mathrm{Na}]^{+} 262.0838$, found 262.0852. The data are in agreement with those previously reported in the literature. ${ }^{7}$

\section{3-Hydroxy-3-phenyl-7-(trifluoromethyl)indolin-2-one $5 f$}<smiles>O=C1Nc2c(C(F)(F)F)cccc2C1(O)c1ccccc1</smiles> 
General procedure B was followed with 7-(trifluoromethyl)isatin $(0.30 \mathrm{~g}, 1.39 \mathrm{mmol})$ and phenylmagnesium bromide ( $3.0 \mathrm{M}^{\text {in }} \mathrm{Et}_{2} \mathrm{O}, 0.93 \mathrm{~mL}, 2.79 \mathrm{mmol}, 2.0$ equiv.). Purification was performed with flash column chromatography over silica gel $\left(40 \mathrm{~g} \mathrm{SiO}_{2}\right.$, heptane/EtOAc, 100/0 to 50/50, gradient) to afford $\mathbf{5 f}$ as a light yellow solid $(0.20 \mathrm{~g}, 0.70 \mathrm{mmol}, 50 \%) .{ }^{\mathbf{1}} \mathbf{H}$ NMR (600 MHz, DMSO-d6): $\delta 10.92$ (brs, $1 \mathrm{H}), 7.56$ (d, $J=8.0 \mathrm{~Hz}, 1 \mathrm{H}), 7.38$ (d, $J=7.3 \mathrm{~Hz}$, 1H), 7.36-7.31 (m, 2H), 7.31-7.24 (m, 3H), $7.16(\mathrm{t}, J=7.7 \mathrm{~Hz}, 1 \mathrm{H}), 6.85(\mathrm{~s}, 1 \mathrm{H}) .{ }^{13} \mathbf{C ~ N M R}$ (150 MHz, CDCl3): $178.8(\mathrm{C}=\mathrm{O}), 140.6(\mathrm{C}), 139.4\left(\mathrm{q}, J^{\mathrm{CF}}=2.2 \mathrm{~Hz}, \mathrm{C}\right), 135.6(\mathrm{C}), 128.9$ $(\mathrm{CH}), 128.3(2 \mathrm{CH}), 127.8(\mathrm{CH}), 125.7\left(\mathrm{q}, J^{\mathrm{CF}}=4.6 \mathrm{~Hz}, \mathrm{CH}\right), 125.4(2 \mathrm{CH}), 123.6\left(\mathrm{q}, J^{\mathrm{CF}}=\right.$ $271.8 \mathrm{~Hz}, \mathrm{C}), 122.4(\mathrm{CH}), 111.0\left(\mathrm{q}, J^{\mathrm{CF}}=32.8 \mathrm{~Hz}, \mathrm{C}\right), 76.0(\mathrm{C}) .{ }^{19} \mathbf{F}$ NMR (471 MHz, CDCl3): $\delta$-59.9. FT-IR (neat, $\mathbf{~ c m}^{-1}$ ): 3219, 2921, 2851, 2360, 2342, 1720, 1614, 1443, 1317 , 1198. HRMS (ESI $\left.{ }^{+}\right): m / z$ calcd. for $\mathrm{C}_{15} \mathrm{H}_{10} \mathrm{~F}_{3} \mathrm{NO}_{2} \mathrm{Na}[\mathrm{M}+\mathrm{Na}]^{+}$316.0556, found 316.0556. 


\section{Characterization data of 3,1-Benzoxazin-2-ones 2a-g, 4a-1 and 6a-f}

4-Methoxy-4-phenyl-1H-benzo[d][1,3]oxazin-2(4H)-one 2a<smiles>COC1(c2ccccc2)OC(=O)Nc2ccccc21</smiles>

$2 a$

General procedure A was followed with $1(90.1 \mathrm{mg}, 0.40 \mathrm{mmol})$ and $\mathrm{MeOH}(2.5 \mathrm{~mL})$. Purification was performed with flash column chromatography over silica gel $\left(20 \mathrm{~g} \mathrm{SiO}_{2}\right.$, heptane/EtOAc, 100/0 to 50/50, gradient) to afford 2a as a white solid (92.7 mg, $0.36 \mathrm{mmol}$,

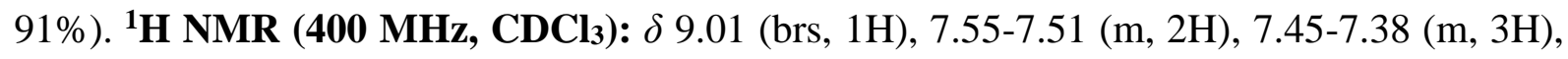
7.34 (ddd, $J=8.4,7.4,1.8 \mathrm{~Hz}, 1 \mathrm{H}), 7.08-7.01(\mathrm{~m}, 2 \mathrm{H}), 6.95(\mathrm{~d}, J=8.4 \mathrm{~Hz}, 1 \mathrm{H}), 3.44(\mathrm{~s}, 3 \mathrm{H})$. ${ }^{13}$ C NMR (100 MHz, CDCl $): \delta 151.6(\mathrm{C}=\mathrm{O}), 138.6(\mathrm{C}), 135.1(\mathrm{C}), 130.6(\mathrm{CH}), 129.3(\mathrm{CH})$, $128.6(2 \mathrm{CH}), 126.9(3 \mathrm{CH}), 123.7(\mathrm{CH}), 121.0(\mathrm{C}), 114.8(\mathrm{CH}), 107.5(\mathrm{C}), 52.0\left(\mathrm{CH}_{3}\right)$. FT-IR (neat, $\mathbf{c m}^{-1}$ ): 1709, 1603, 1492, 1449, 1434, 1346, 1256, 1215, 1177, 1093, 1076, 1007. HRMS (ESI $\left.{ }^{+}\right): m / z$ calcd. for $\mathrm{C}_{15} \mathrm{H}_{13} \mathrm{NO}_{3} \mathrm{Na}[\mathrm{M}+\mathrm{Na}]^{+} 278.0788$, found 278.0789.

\section{4-Ethoxy-4-phenyl-1H-benzo[d][1,3]oxazin-2(4H)-one $2 \mathrm{~b}$}<smiles>CCOC1(c2ccccc2)OC(=O)Nc2ccccc21</smiles>

General procedure A was followed with 1 (90.1 $\mathrm{mg}, 0.40 \mathrm{mmol})$ and $\mathrm{EtOH}(2.5 \mathrm{~mL})$. Purification was performed with flash column chromatography over silica gel $\left(20 \mathrm{~g} \mathrm{SiO}_{2}\right.$, heptane/EtOAc, 100/0 to 50/50, gradient) to afford $\mathbf{2 b}$ as a white solid (83.3 $\mathrm{mg}, 0.31 \mathrm{mmol}$, 77\%). ${ }^{1} \mathbf{H}$ NMR (400 MHz, CDCl 3 ): $\delta 9.73$ (brs, $\left.1 \mathrm{H}\right), 7.59-7.52(\mathrm{~m}, 2 \mathrm{H}), 7.44-7.37$ (m, 3H), 7.32 (ddd, $J=8.4,7.4,1.8 \mathrm{~Hz}, 1 \mathrm{H}), 7.08-7.02(\mathrm{~m}, 2 \mathrm{H}), 6.99$ (d, $J=8.4 \mathrm{~Hz}, 1 \mathrm{H}), 3.71$ (qd, $J=$ 7.1, $2.2 \mathrm{~Hz}, 2 \mathrm{H}), 1.28(\mathrm{t}, J=7.1 \mathrm{~Hz}, 3 \mathrm{H}) .{ }^{13} \mathbf{C} \mathbf{N M R}\left(\mathbf{1 0 0} \mathbf{M H z}, \mathbf{C D C l}_{3}\right): \delta 152.3(\mathrm{C}=\mathrm{O})$, $139.3(\mathrm{C}), 136.0(\mathrm{C}), 130.4(\mathrm{CH}), 129.2(\mathrm{CH}), 128.5(2 \mathrm{CH}), 126.8(2 \mathrm{CH}), 126.8(\mathrm{CH}), 123.6$ $(\mathrm{CH}), 121.3(\mathrm{C}), 114.9(\mathrm{CH}), 107.5(\mathrm{C}), 60.5\left(\mathrm{CH}_{2}\right), 15.4\left(\mathrm{CH}_{3}\right)$. FT-IR (neat, $\left.\mathbf{c m}^{-1}\right)$ : 1710, 1601, 1490, 1449, 1342, 1255, 1215, 1135, 1095, 1076, 1026, 1002. HRMS (ESI $\left.{ }^{+}\right)$: $m / z$ calcd. for $\mathrm{C}_{16} \mathrm{H}_{15} \mathrm{NO}_{3} \mathrm{Na}[\mathrm{M}+\mathrm{Na}]^{+} 292.0944$, found 292.0941 . 
<smiles>CCCOC1(c2ccccc2)OC(=O)Nc2ccccc21</smiles>

General procedure A was followed with $1(90.1 \mathrm{mg}, 0.40 \mathrm{mmol})$ and $n \operatorname{PrOH}(2.5 \mathrm{~mL})$. Purification was performed with flash column chromatography over silica gel $\left(20 \mathrm{~g} \mathrm{SiO}_{2}\right.$, heptane/EtOAc, 100/0 to 50/50, gradient) to afford $\mathbf{2 c}$ as a white solid (89.3 $\mathrm{mg}, 0.32 \mathrm{mmol}$,

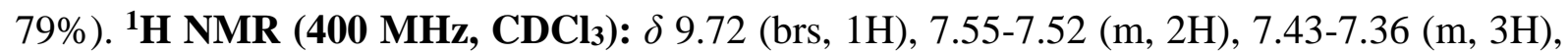
$7.30(\mathrm{ddd}, J=8.4,7.4,1.8 \mathrm{~Hz}, 1 \mathrm{H}), 7.08-7.00(\mathrm{~m}, 2 \mathrm{H}), 6.99(\mathrm{~d}, J=8.4 \mathrm{~Hz}, 1 \mathrm{H}), 3.58(\mathrm{qt}, J=$ 8.8, $7.4 \mathrm{~Hz}, 2 \mathrm{H}), 1.73-1.63(\mathrm{~m}, 2 \mathrm{H}), 0.95$ (t, $J=7.4 \mathrm{~Hz}, 3 \mathrm{H}) .{ }^{13} \mathbf{C}$ NMR (100 MHz, CDCl 3$): \delta$ $152.2(\mathrm{C}=\mathrm{O}), 139.6(\mathrm{C}), 136.1(\mathrm{C}), 130.4(\mathrm{CH}), 129.2(\mathrm{CH}), 128.5(2 \mathrm{CH}), 126.9(\mathrm{CH}), 126.8$ $(2 \mathrm{CH}), 123.6(\mathrm{CH}), 121.2(\mathrm{C}), 114.9(\mathrm{CH}), 107.4(\mathrm{C}), 66.2\left(\mathrm{CH}_{2}\right), 23.1\left(\mathrm{CH}_{2}\right), 10.7\left(\mathrm{CH}_{3}\right)$. FT-IR (neat, $\mathbf{c m}^{-1}$ ): 1708, 1601, 1491, 1449, 1434, 1341, 1254, 1216, 1180, 1133, 1094, 1077, 1036, 1002. HRMS (ESI $\left.{ }^{+}\right): m / z$ calcd. for $\mathrm{C}_{17} \mathrm{H}_{17} \mathrm{NO}_{3} \mathrm{Na}[\mathrm{M}+\mathrm{Na}]^{+}$306.1101, found 306.1102 .

4-(Benzyloxy)-4-phenyl-1H-benzo[d][1,3]oxazin-2(4H)-one 2d<smiles>O=C1Nc2ccccc2C(Br)(c2ccccc2)OC1c1ccccc1</smiles>

2d

The general procedure A was followed with 1 (90.1 mg, $0.40 \mathrm{mmol})$ and $\mathrm{BzOH}(2.5 \mathrm{~mL})$. Purification was performed with flash column chromatography over silica gel $\left(20 \mathrm{~g} \mathrm{SiO}_{2}\right.$, heptane/EtOAc, 100/0 to 50/50, gradient) to afford $2 \mathrm{~d}$ as a white solid (64.6 mg, $0.12 \mathrm{mmol}$, 49\%). ${ }^{1} \mathbf{H}$ NMR (400 MHz, CDCl 3 ): $\delta 9.77$ (brs, $\left.1 \mathrm{H}\right), 7.54-7.47$ (m, 2H), 7.36-7.29 (m, 3H), 7.28-7.10 (m, 6H), $7.04(\mathrm{~d}, J=7.5 \mathrm{~Hz}, 1 \mathrm{H}), 6.98-6.87(\mathrm{~m}, 2 \mathrm{H}), 4.62(\mathrm{~d}, J=2.7 \mathrm{~Hz}, 2 \mathrm{H}) .{ }^{13} \mathbf{C}$ NMR (100 MHz, CDCl3): $\delta 152.1(\mathrm{C}=\mathrm{O}), 139.1$ (C), $137.1(\mathrm{C}), 135.1(\mathrm{C}), 130.6(\mathrm{CH}), 129.4$ $(\mathrm{CH}), 128.6(2 \mathrm{CH}), 128.5(2 \mathrm{CH}), 127.9(3 \mathrm{CH}), 126.9(3 \mathrm{CH}), 123.7(\mathrm{CH}), 120.9(\mathrm{C}), 115.0$ $(\mathrm{CH}), 107.5(\mathrm{C}), 66.6\left(\mathrm{CH}_{2}\right)$. HRMS (ESI $\left.{ }^{+}\right): \mathrm{m} / z$ calcd. for $\mathrm{C}_{21} \mathrm{H}_{17} \mathrm{NO}_{3} \mathrm{Na}[\mathrm{M}+\mathrm{Na}]^{+} 354.1101$, found 354.1101 .

4-Phenethoxy-4-phenyl-1H-benzo[d][1,3]oxazin-2(4H)-one $2 \mathrm{e}$<smiles>O=C1Nc2ccccc2C(OCCc2ccccc2)(c2ccccc2)O1</smiles> 
General procedure A was followed with 1 (90.1 $\mathrm{mg}, 0.40 \mathrm{mmol})$ and 2-phenylethanol $(2.5 \mathrm{~mL})$. Purification was performed with flash column chromatography over silica gel $(20 \mathrm{~g}$ $\mathrm{SiO}_{2}$, heptane/EtOAc, $100 / 0$ to $50 / 50$, gradient) to afford $2 \mathrm{e}$ as a white solid $(46.2 \mathrm{mg}$, 0.14 mmol, 33\%). ${ }^{1} \mathbf{H}$ NMR (400 MHz, CDCl $): \delta 9.24$ (brs, $\left.1 \mathrm{H}\right), 7.39-7.34$ (m, 2H), 7.31$7.27(\mathrm{~m}, 3 \mathrm{H}), 7.24-7.17(\mathrm{~m}, 3 \mathrm{H}), 7.14-7.09(\mathrm{~m}, 3 \mathrm{H}), 6.91(\mathrm{ddd}, J=8.4,7.4,1.8 \mathrm{~Hz}, 1 \mathrm{H})$, 6.88-6.84 (m, 2H), 3.82-3.72 (m, 2H), $2.90(\mathrm{t}, J=7.1 \mathrm{~Hz}, 2 \mathrm{H}) .{ }^{13} \mathbf{C}$ NMR (100 MHz, CDCl3): $\delta 151.8(\mathrm{C}=\mathrm{O}), 139.3(\mathrm{C}), 138.5(\mathrm{C}), 136.0(\mathrm{C}), 130.4(\mathrm{CH}), 129.2(2 \mathrm{CH}), 129.2$ $(\mathrm{CH}), 128.6(2 \mathrm{CH}), 128.5(2 \mathrm{CH}), 126.8(\mathrm{CH}), 126.8(2 \mathrm{CH}), 126.5(\mathrm{CH}), 123.6(\mathrm{CH}), 121.0$ (C), $114.8(\mathrm{CH}), 107.4(\mathrm{C}), 66.5\left(\mathrm{CH}_{2}\right), 36.4\left(\mathrm{CH}_{2}\right)$. HRMS $\left(\mathbf{E S I}^{+}\right): \mathrm{m} / z$ calcd. for $\mathrm{C}_{22} \mathrm{H}_{19} \mathrm{NO}_{3} \mathrm{Na}[\mathrm{M}+\mathrm{Na}]^{+}$368.1257, found 368.1256.

\section{4-(Allyloxy)-4-phenyl-1H-benzo[d][1,3]oxazin-2(4H)-one $2 \mathrm{f}$}<smiles>C=CCOC1(c2ccccc2)OC(=O)Nc2ccccc21</smiles>

General procedure A was followed with $1(90.1 \mathrm{mg}, 0.40 \mathrm{mmol})$ and allyl alcohol $(2.5 \mathrm{~mL})$. Purification was performed with flash column chromatography over silica gel $\left(20 \mathrm{~g} \mathrm{SiO}_{2}\right.$, heptane/EtOAc, 100/0 to 50/50, gradient) to afford $\mathbf{2 f}$ as a white solid (86.6 mg, $0.31 \mathrm{mmol}$, 77\%). ${ }^{1} \mathbf{H}$ NMR (400 MHz, CDCl $): ~ \delta 9.31$ (brs, $\left.1 \mathrm{H}\right), 7.57-7.53(\mathrm{~m}, 2 \mathrm{H}), 7.44-7.38(\mathrm{~m}, 3 \mathrm{H})$, 7.31 (ddd, $J=8.4,7.4,1.8 \mathrm{~Hz}, 1 \mathrm{H}), 7.08-7.00(\mathrm{~m}, 2 \mathrm{H}), 6.96(\mathrm{~d}, J=8.4 \mathrm{~Hz}, 1 \mathrm{H}), 5.96$ (ddt, $J$ $=17.2,10.5,5.5 \mathrm{~Hz}, 1 \mathrm{H}), 5.35-5.29(\mathrm{~m}, 1 \mathrm{H}), 5.21-5.16(\mathrm{~m}, 1 \mathrm{H}), 4.18(\mathrm{ddd}, J=5.5,1.7,1.6$

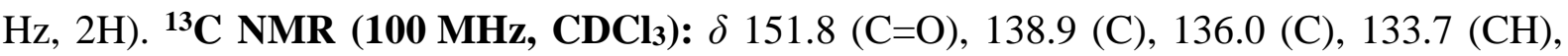
$130.5(\mathrm{CH}), 129.3(\mathrm{CH}), 128.6(2 \mathrm{CH}), 126.9(3 \mathrm{CH}), 123.7(\mathrm{CH}), 121.2(\mathrm{C}), 117.5\left(\mathrm{CH}_{2}\right)$, $114.9(\mathrm{CH}), 107.4(\mathrm{C}), 66.9\left(\mathrm{CH}_{2}\right)$. FT-IR (neat, $\left.\mathbf{c m}^{-1}\right)$ : 1709, 1602, 1491, 1449, 1347, 1256, 1213, 1133, 1105, 1090, 1074, 1036, 1005. HRMS (ESI $\left.{ }^{+}\right): \mathrm{m} / \mathrm{z}$ calcd. for $\mathrm{C}_{17} \mathrm{H}_{15} \mathrm{NO}_{3} \mathrm{Na}$ $[\mathrm{M}+\mathrm{Na}]^{+}$304.0944, found 304.0942.

\section{4-Isopropoxy-4-phenyl-1H-benzo[d][1,3]oxazin-2(4H)-one $2 \mathrm{~g}$}<smiles>CC(C)OC1(c2ccccc2)OC(=O)Nc2ccccc21</smiles>

$2 g$

General procedure A was followed with 1 (90.1 mg, $0.40 \mathrm{mmol})$ and $i \mathrm{PrOH}(2.5 \mathrm{~mL})$. Purification was performed with flash column chromatography over silica gel $\left(20 \mathrm{~g} \mathrm{SiO}_{2}\right.$, heptane/EtOAc, 100/0 to 50/50, gradient) to afford $\mathbf{2 g}$ as a brown solid (57.8 $\mathrm{mg}, 0.20 \mathrm{mmol}$, 51\%). ${ }^{1} \mathbf{H}$ NMR (400 MHz, CDCl3): $\delta 9.79$ (brs, $\left.1 \mathrm{H}\right), 7.57-7.50(\mathrm{~m}, 2 \mathrm{H}), 7.42-7.35$ (m, 3H), 7.29 (ddd, $J=7.6,7.6,1.4 \mathrm{~Hz}, 1 \mathrm{H}), 7.15$ (dd, $J=7.6,1.4 \mathrm{~Hz}, 1 \mathrm{H}), 7.02$ (ddd, $J=7.6,7.6,1.1$ 
$\mathrm{Hz}, 1 \mathrm{H}), 6.98(\mathrm{dd}, J=7.6,1.1 \mathrm{~Hz}, 1 \mathrm{H}) 4.12-4.02(\mathrm{~m}, 1 \mathrm{H}), 1.30(\mathrm{~d}, J=6.1 \mathrm{~Hz}, 3 \mathrm{H}), 1.23(\mathrm{~d}, J$ $=6.1 \mathrm{~Hz}, 3 \mathrm{H}) .{ }^{13} \mathbf{C}$ NMR (100 MHz, CDCl$): \delta 152.3(\mathrm{C}=\mathrm{O}), 140.2(\mathrm{C}), 135.0(\mathrm{C}), 130.4$ $(\mathrm{CH}), 129.1(\mathrm{CH}), 128.4(2 \mathrm{CH}), 127.1(\mathrm{CH}), 126.9(2 \mathrm{CH}), 123.4(\mathrm{CH}), 121.4(\mathrm{C}), 114.9$ $(\mathrm{CH}), 108.3(\mathrm{C}), 69.2(\mathrm{CH}), 24.2\left(2 \mathrm{CH}_{3}\right)$. FT-IR (neat, $\left.\mathbf{c m}^{-1}\right)$ : 1707, 1599, 1494, 1361, 1258, 1202, 1068, 1012. HRMS (ESI $\left.{ }^{+}\right): m / z$ calcd. for $\mathrm{C}_{17} \mathrm{H}_{17} \mathrm{NO}_{3} \mathrm{Na}[\mathrm{M}+\mathrm{Na}]^{+}$306.1101, found 306.1105 .

\section{4-Methoxy-4-(4-methoxyphenyl)-1H-benzo[d][1,3]oxazin-2(4H)-one 4a}<smiles>COc1ccc(C2(OC)OC(=O)Nc3ccccc32)cc1</smiles>

The general procedure A was followed with 3a (102 mg, $0.40 \mathrm{mmol})$ and $\mathrm{MeOH}(2.5 \mathrm{~mL})$. Purification was performed with flash column chromatography over silica gel $\left(20 \mathrm{~g} \mathrm{SiO}_{2}\right.$, heptane/EtOAc, 100/0 to 50/50, gradient) to afford 4a as a light brown solid $(98.7 \mathrm{mg}$, 0.35 mmol, 87\%). ${ }^{\mathbf{1}} \mathbf{H}$ NMR (400 MHz, CDCl3): $\delta 8.79$ (brs, $\left.1 \mathrm{H}\right), 7.43$ (d, $\left.J=8.9 \mathrm{~Hz}, 2 \mathrm{H}\right)$, 7.31 (ddd, $J=8.0,7.0,1.9 \mathrm{~Hz}, 1 \mathrm{H}), 7.10-7.00(\mathrm{~m}, 2 \mathrm{H}), 6.94-6.90(\mathrm{~m}, 3 \mathrm{H}), 3.82(\mathrm{~s}, 3 \mathrm{H}), 3.43$ (s, 3H). ${ }^{13}$ C NMR (100 MHz, CDCl3): $\delta 160.3$ (C=O), 151.6 (C), 136.1 (C), 130.9 (C), 130.5 $(\mathrm{CH}), 128.4(2 \mathrm{CH}), 126.9(\mathrm{CH}), 123.6(\mathrm{CH}), 121.3(\mathrm{C}), 114.7(\mathrm{CH}), 113.9(2 \mathrm{CH}), 107.6(\mathrm{C})$, $55.5\left(\mathrm{CH}_{3}\right), 52.0\left(\mathrm{CH}_{3}\right)$. FT-IR (neat, $\left.\mathbf{c m}^{-1}\right)$ : 1700, 1604, 1513, 1505, 1494, 1432, 1353, 1319, 1305, 1251, 1209, 1185, 1169, 1153, 1095, 1032, 1009. HRMS (ESI $\left.{ }^{+}\right): \mathrm{m} / z$ calcd. for $\mathrm{C}_{16} \mathrm{H}_{15} \mathrm{NO}_{4} \mathrm{Na}[\mathrm{M}+\mathrm{Na}]^{+}$308.0893, found 308.0887.

\section{4-Methoxy-4-(p-tolyl)-1H-benzo[d][1,3]oxazin-2(4H)-one 4b}<smiles>COC1(c2ccc(C)cc2)OC(=O)Nc2ccccc21</smiles>

4b

General procedure A was followed with $\mathbf{3 b}(95.7 \mathrm{mg}, 0.40 \mathrm{mmol})$ and $\mathrm{MeOH}(2.5 \mathrm{~mL})$ Purification was performed with flash column chromatography over silica gel $\left(20 \mathrm{~g} \mathrm{SiO}_{2}\right.$, heptane/EtOAc, 100/0 to 50/50, gradient) to afford $\mathbf{4 b}$ as a light yellow solid (99.0 $\mathrm{mg}, 0.37$ mmol, 92\%). ${ }^{1} \mathbf{H}$ NMR (400 MHz, CDCl 3$): \delta 9.66$ (brs, $\left.1 \mathrm{H}\right), 7.40$ (d, $\left.J=8.3 \mathrm{~Hz}, 2 \mathrm{H}\right), 7.30$ $(\mathrm{td}, J=8.0,1.5 \mathrm{~Hz}, 1 \mathrm{H}), 7.22(\mathrm{~d}, J=8.0 \mathrm{~Hz}, 2 \mathrm{H}), 7.06(\mathrm{dd}, J=7.7,1.5 \mathrm{~Hz}, 1 \mathrm{H}), 7.02(\mathrm{td}, J=$ 7.7, $1.0 \mathrm{~Hz}, 1 \mathrm{H}), 6.99(\mathrm{dd}, J=8.0,1.0 \mathrm{~Hz}, 1 \mathrm{H}), 3.43(\mathrm{~s}, 3 \mathrm{H}), 2.37$ (s, 3H). ${ }^{13} \mathrm{C}$ NMR 
(150 MHz, CDCl 3$): \delta 152.3(\mathrm{C}=\mathrm{O}), 139.3(\mathrm{C}), 135.9(\mathrm{C}), 135.2(\mathrm{C}), 130.5(\mathrm{CH}), 129.3$ $(2 \mathrm{CH}), 126.9(2 \mathrm{CH}), 126.8(\mathrm{CH}), 123.7(\mathrm{CH}), 121.1(\mathrm{C}), 115.0(\mathrm{CH}), 107.7(\mathrm{C}), 52.0\left(\mathrm{CH}_{3}\right)$, $21.4\left(\mathrm{CH}_{3}\right)$. FT-IR (neat, $\left.\mathbf{c m}^{-1}\right): 3096,3004,2924,2360,2342,1714,1603,1493,1345$, 1255, 1182. HRMS (ESI $\left.{ }^{+}\right): m / z$ calcd. for $\mathrm{C}_{16} \mathrm{H}_{15} \mathrm{NO}_{3} \mathrm{Na}[\mathrm{M}+\mathrm{Na}]^{+}$292.0944, found 292.0943.

\section{4-(4-(Tert-butyl)phenyl)-4-methoxy-1H-benzo[d][1,3]oxazin-2(4H)-one 4c}<smiles>COC1(c2ccc(C(C)(C)C)cc2)OC(=O)Nc2ccccc21</smiles>

The general procedure A was followed with $3 \mathbf{c}(113 \mathrm{mg}, 0.40 \mathrm{mmol})$ and $\mathrm{MeOH}(2.5 \mathrm{~mL})$. Purification was performed with flash column chromatography over silica gel $\left(20 \mathrm{~g} \mathrm{SiO}_{2}\right.$, heptane/EtOAc, 100/0 to 50/50, gradient) to afford $\mathbf{4 c}$ as a light yellow solid $(89.9 \mathrm{mg}$, 0.29 mmol, 72\%). ${ }^{1}$ H NMR (600 MHz, DMSO-d6): $\delta 10.62$ (brs, $\left.1 \mathrm{H}\right), 7.46$ (d, $J=8.5 \mathrm{~Hz}$, 2H), 7.36-7.29 (m, 3H), $7.02(\mathrm{t}, J=7.5 \mathrm{~Hz}, 1 \mathrm{H}), 7.00-6.95(\mathrm{~m}, 2 \mathrm{H}), 3.25(\mathrm{~s}, 3 \mathrm{H}), 1.27(\mathrm{~s}, 9 \mathrm{H})$. ${ }^{13}$ C NMR (150 MHz, DMSO-d6): $\delta 151.5$ (C=O), 149.3 (C), 136.7 (C), 135.8 (C), 130.3 $(\mathrm{CH}), 126.3(\mathrm{CH}), 125.9(2 \mathrm{CH}), 125.3(2 \mathrm{CH}), 122.7(\mathrm{CH}), 120.1(\mathrm{C}), 114.4(\mathrm{CH}), 106.0(\mathrm{C})$, $51.0\left(\mathrm{CH}_{3}\right), 34.4(\mathrm{C}), 31.0\left(3 \mathrm{CH}_{3}\right)$. FT-IR (neat, $\left.\mathbf{c m}^{-1}\right)$ : 3100, 2950, 2359, 2342, 1720, 1602, 1494, 1347, 1275, 1263, 1258. HRMS (ESI $\left.{ }^{+}\right): m / z$ calcd. for $\mathrm{C}_{19} \mathrm{H}_{22} \mathrm{NO}_{3}[\mathrm{M}+\mathrm{H}]^{+} 312.1594$, found 312.1595 .

4-([1,1'-Biphenyl]-4-yl)-4-methoxy-1H-benzo[d][1,3]oxazin-2(4H)-one 4d<smiles>COC1(c2ccc(-c3ccccc3)cc2)OC(=O)Nc2ccccc21</smiles>

4d

The general procedure A was followed was followed with 3d $(121 \mathrm{mg}, 0.40 \mathrm{mmol})$ and $\mathrm{MeOH}(2.5 \mathrm{~mL})$. Purification was performed with flash column chromatography over silica gel (20 $\mathrm{g} \mathrm{SiO}_{2}$, heptane/EtOAc, 100/0 to 50/50, gradient) to afford $\mathbf{4 d}$ as a light yellow solid (91.1 mg, $0.28 \mathrm{mmol}, 69 \%) .{ }^{1}$ H NMR (600 MHz, DMSO-d6): $\delta 10.69$ (brs, 1H), 7.74 (d, $J=$ $8.4 \mathrm{~Hz}, 2 \mathrm{H}), 7.68(\mathrm{~d}, J=7.3 \mathrm{~Hz}, 2 \mathrm{H}), 7.50(\mathrm{~d}, J=8.4 \mathrm{~Hz}, 2 \mathrm{H}), 7.48(\mathrm{t}, J=7.7 \mathrm{~Hz}, 2 \mathrm{H}), 7.39$ (t, $J=7.3 \mathrm{~Hz}, 1 \mathrm{H}), 7.35$ (ddd, $J=8.6,6.1,2.8 \mathrm{~Hz}, 1 \mathrm{H}), 7.08-7.02(\mathrm{~m}, 2 \mathrm{H}), 7.00(\mathrm{~d}, J=8.1$ 
Hz, 1H), 3.29 (s, 3H). ${ }^{13}$ C NMR (150 MHz, DMSO-d6): $\delta 149.2$ (C=O), 140.8 (C), 139.3 (C), $138.6(\mathrm{C}), 135.8(\mathrm{C}), 130.4(\mathrm{CH}), 129.0(2 \mathrm{CH}), 127.8(\mathrm{CH}), 126.8(2 \mathrm{CH}), 126.8(2 \mathrm{CH})$, $126.8(2 \mathrm{CH}), 126.3(\mathrm{CH}), 122.8(\mathrm{CH}), 119.9(\mathrm{C}), 114.5(\mathrm{CH}), 105.9(\mathrm{C}), 51.0\left(\mathrm{CH}_{3}\right)$. FT-IR (neat, $\mathbf{c m}^{-1}$ ): 3006, 2989, 2360, 2342, 1716, 1603, 1492, 1343, 1275, 1258, 1216, 1186. HRMS (ESI $\left.{ }^{+}\right): m / z$ calcd. for $\mathrm{C}_{21} \mathrm{H}_{17} \mathrm{NO}_{3} \mathrm{Na}[\mathrm{M}+\mathrm{Na}]^{+} 354.1101$, found 354.1097.

4-(4-Chlorophenyl)-4-methoxy-1H-benzo[d][1,3]oxazin-2(4H)-one 4e<smiles>COC1(c2ccc(Cl)cc2)OC(=O)Nc2ccccc21</smiles>

General procedure A was followed with $3 \mathbf{e}(104 \mathrm{mg}, 0.40 \mathrm{mmol})$ and $\mathrm{MeOH}(2.5 \mathrm{~mL})$. Purification was performed with flash column chromatography over silica gel $\left(20 \mathrm{~g} \mathrm{SiO}_{2}\right.$, heptane/EtOAc, 100/0 to 50/50, gradient) to afford $4 \mathbf{e}$ as a light yellow solid (60.0 $\mathrm{mg}, 0.21$ mmol, 52\%). ${ }^{1} \mathbf{H}$ NMR (600 MHz, CDCl 3$): \delta 9.66(\mathrm{~s}, 1 \mathrm{H}), 7.46(\mathrm{~d}, J=8.6 \mathrm{~Hz}, 2 \mathrm{H}), 7.38(\mathrm{~d}, J$ $=8.6 \mathrm{~Hz}, 2 \mathrm{H}), 7.33(\mathrm{td}, J=7.6,1.5 \mathrm{~Hz}, 1 \mathrm{H}), 7.07-7.03(\mathrm{~m}, 1 \mathrm{H}), 7.03-6.97(\mathrm{~m}, 2 \mathrm{H}), 3.42(\mathrm{~s}$, 3H). ${ }^{13} \mathrm{C}$ NMR (150 MHz, CDCl3): $\delta 151.8(\mathrm{C}=\mathrm{O}), 137.5$ (C), $135.4(\mathrm{C}), 135.1$ (C), 130.8 $(\mathrm{CH}), 128.8(2 \mathrm{CH}), 128.4(2 \mathrm{CH}), 126.8(\mathrm{CH}), 123.8(\mathrm{CH}), 120.1(\mathrm{C}), 115.0(\mathrm{CH}), 107.1(\mathrm{C})$, $52.0\left(\mathrm{CH}_{3}\right)$. FT-IR (neat, $\left.\mathbf{c m}^{-1}\right)$ : 3093, 2989, 2925, 2359, 2342, 1703, 1601, 1491, 1343, 1267, 1253, 1219. HRMS (ESI $\left.{ }^{+}\right): m / z$ calcd. for $\mathrm{C}_{15} \mathrm{H}_{12} \mathrm{ClNO}_{3} \mathrm{Na}[\mathrm{M}+\mathrm{Na}]^{+} 312.0398$, found 312.0398 .

4-(4-Fluoro-phenyl)-4-methoxy-1,4-dihydro-benzo[d][1,3]oxazin-2-one 4f<smiles>COC1(c2ccc(F)cc2)OC(=O)Nc2ccccc21</smiles>

$4 \mathbf{f}$

General procedure A was followed with $3 \mathbf{f}(97.3 \mathrm{mg}, 0.40 \mathrm{mmol})$ and $\mathrm{MeOH}(2.5 \mathrm{~mL})$. Purification was performed with flash column chromatography over silica gel $\left(20 \mathrm{~g} \mathrm{SiO}_{2}\right.$, heptane/EtOAc, 100/0 to 50/50, gradient) to afford $\mathbf{4 f}$ as a light yellow solid (88.5 $\mathrm{mg}, 0.32$ mmol, 81\%). ${ }^{1} \mathbf{H}$ NMR (700 MHz, DMSO-d6): $\delta 10.70$ (brs, 1H), 7.48-7.45 (m, 2H), 7.35 $(\mathrm{dd}, J=7.8,1.5 \mathrm{~Hz}, 1 \mathrm{H}), 7.29-7.25(\mathrm{~m}, 2 \mathrm{H}), 7.02(\mathrm{dd}, J=8.0,1.1 \mathrm{~Hz}, 1 \mathrm{H}), 6.99$ (d, $J=8.0$ $\mathrm{Hz}, 1 \mathrm{H}), 6.94$ (dd, $J=7.8,1.1 \mathrm{~Hz}, 1 \mathrm{H}), 3.25$ (s, 3H). ${ }^{13} \mathrm{C}$ NMR (176 MHz, DMSO): $\delta 162.7$ $\left(\mathrm{d}, J^{\mathrm{CF}}=245.8 \mathrm{~Hz}, \mathrm{C}\right), 149.1(\mathrm{C}=\mathrm{O}), 135.8\left(\mathrm{~d}, J^{\mathrm{CF}}=4.0 \mathrm{~Hz}, \mathrm{C}\right), 135.8(\mathrm{C}), 130.5(\mathrm{CH}), 128.6$ $\left(\mathrm{d}, J^{\mathrm{CF}}=8.6 \mathrm{~Hz}, 2 \mathrm{CH}\right), 126.3(\mathrm{CH}), 122.8(\mathrm{CH}), 119.7(\mathrm{C}), 115.4\left(\mathrm{~d}, J^{\mathrm{CF}}=21.7 \mathrm{~Hz}, 2 \mathrm{CH}\right)$, 
$114.5(\mathrm{CH}), 105.5(\mathrm{C}), 51.0\left(\mathrm{CH}_{3}\right) .{ }^{19} \mathbf{F}$ NMR (659 MHz, DMSO-d6): $\delta$-113.0. FT-IR (neat, $\mathbf{c m}^{-1}$ ): 3094, 2990, 2930, 2360, 2332, 1716, 1708, 1602, 1507, 1494, 1345, 1276, 1228, 1217. HRMS (ESI $\left.{ }^{+}\right): m / z$ calcd. for $\mathrm{C}_{15} \mathrm{H}_{12} \mathrm{FNO}_{3} \mathrm{Na}[\mathrm{M}+\mathrm{Na}]^{+} 296.0693$, found 296.0692.

\section{4-Methoxy-4-(3-methoxyphenyl)-1H-benzo[d][1,3]oxazin-2(4H)-one 4g}<smiles>COc1cccc(C2(OC)OC(=O)Nc3ccccc32)c1</smiles>

The general procedure A was followed with $\mathbf{3 g}(102 \mathrm{mg}, 0.40 \mathrm{mmol})$ and $\mathrm{MeOH}(2.5 \mathrm{~mL})$ Purification was performed with flash column chromatography over silica gel $\left(20 \mathrm{~g} \mathrm{SiO}_{2}\right.$, heptane/EtOAc, $100 / 0$ to $50 / 50$, gradient) to afford $\mathbf{4 g}$ as a light brown solid $(61.3 \mathrm{mg}$, 0.22 mmol, 54\%). ${ }^{1} \mathbf{H}$ NMR (600 MHz, CDCl 3$): \delta 9.38$ (brs, $\left.1 \mathrm{H}\right), 7.35-7.29$ (m, 2H), 7.10$7.07(\mathrm{~m}, 3 \mathrm{H}), 7.03(\mathrm{ddd}, J=8.0,7.0,1.9 \mathrm{~Hz}, 1 \mathrm{H}), 6.97(\mathrm{dd}, J=8.0,0.9 \mathrm{~Hz}, 1 \mathrm{H}), 6.93$ (ddd, $J$ $=8.1,2.5,0.9,1 \mathrm{H}), 3.82(\mathrm{~s}, 3 \mathrm{H}), 3.45(\mathrm{~s}, 3 \mathrm{H}) .{ }^{13} \mathbf{C}$ NMR (150 MHz, CDCl 3$): \delta 159.9(\mathrm{C}=\mathrm{O})$, $151.8(\mathrm{C}), 140.0(\mathrm{C}), 135.0(\mathrm{C}), 130.6(\mathrm{CH}), 129.6(\mathrm{CH}), 126.8(\mathrm{CH}), 123.7(\mathrm{CH}), 120.8(\mathrm{C})$, $119.3(\mathrm{CH}), 115.0(\mathrm{CH}), 114.9(\mathrm{CH}), 112.6(\mathrm{CH}), 107.3(\mathrm{C}), 55.5\left(\mathrm{CH}_{3}\right), 52.0\left(\mathrm{CH}_{3}\right)$. FT-IR (neat, $\mathbf{c m}^{-1}$ ): 1702, 1604, 1589, 1486, 1433, 1355, 1313, 1293, 1273, 1263, 1200, 1171, 1086, 1052, 1041, 1015. HRMS (ESI $\left.{ }^{+}\right): m / z$ calcd. for $\mathrm{C}_{16} \mathrm{H}_{15} \mathrm{NO}_{4} \mathrm{Na}[\mathrm{M}+\mathrm{Na}]^{+} 308.0893$, found 308.0898 .

\section{4-Methoxy-4-(perfluorophenyl)-1H-benzo[d][1,3]oxazin-2(4H)-one $4 \mathrm{~h}$}<smiles>COC1(c2c(F)c(F)c(F)c(F)c2F)OC(=O)Nc2ccccc21</smiles>

General procedure A was followed with $3 \mathbf{h}(126 \mathrm{mg}, 0.40 \mathrm{mmol})$ and $\mathrm{MeOH}(2.5 \mathrm{~mL})$. Purification was performed with flash column chromatography over silica gel $\left(20 \mathrm{~g} \mathrm{SiO}_{2}\right.$, heptane/EtOAc, 100/0 to 50/50, gradient) to afford $\mathbf{4 h}$ as a light yellow solid (27.0 $\mathrm{mg}$, 0.078 mmol, 20\%). ${ }^{1} \mathbf{H}$ NMR (700 MHz, CDCl $): \delta 9.07$ (brs, 1H), 7.38-7.35 (m, 1H), 7.13$7.04(\mathrm{~m}, 2 \mathrm{H}), 6.94(\mathrm{~d}, J=8.0 \mathrm{~Hz}, 1 \mathrm{H}), 3.45$ (s, 3H). ${ }^{13} \mathbf{C}$ NMR (176 MHz, CDCl 3$): ~ \delta 149.9$ $(\mathrm{C}=\mathrm{O}), 144.9(\mathrm{dm}, J=257.1 \mathrm{~Hz}, 2 \mathrm{C}), 141.8(\mathrm{dm}, J=257.2 \mathrm{~Hz}, \mathrm{C}), 138.2(\mathrm{dm}, J=251.7 \mathrm{~Hz}$, 2C), $134.5(\mathrm{C}), 131.5(\mathrm{CH}), 126.1(\mathrm{CH}), 124.3(\mathrm{CH}), 118.0(\mathrm{C}), 114.9(\mathrm{CH}), 114.8(\mathrm{~m}, \mathrm{C})$,

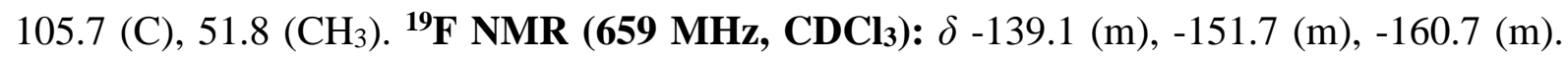
FT-IR (neat, $\left.\mathbf{c m}^{-1}\right)$ : 3240, 3111, 2931, 1716, 1606, 1521, 1489, 1353, 1309, 1273, 1252, 1148. HRMS (ESI $\left.{ }^{+}\right): \mathrm{m} / z$ calcd. for $\mathrm{C}_{15} \mathrm{H}_{8} \mathrm{~F}_{5} \mathrm{NO}_{3} \mathrm{Na}[\mathrm{M}+\mathrm{Na}]^{+} 368.0317$, found 368.0317 
<smiles>COC1(C)OC(=O)Nc2ccccc21</smiles>

General procedure A was followed with $3 \mathbf{i}(65.3 \mathrm{mg}, 0.40 \mathrm{mmol})$ and $\mathrm{MeOH}(2.5 \mathrm{~mL})$. Purification was performed with flash column chromatography over silica gel $\left(20 \mathrm{~g} \mathrm{SiO}_{2}\right.$, heptane/EtOAc, $100 / 0$ to $50 / 50$, gradient) to afford $4 \mathbf{i}$ as a light yellow solid (37.8 $\mathrm{mg}$, 0.20 mmol, 49\%). ${ }^{1} \mathbf{H}$ NMR (400 MHz, CDCl3): $\delta 9.81(\mathrm{~s}, 1 \mathrm{H}), 7.31(\mathrm{td}, J=7.7,1.4 \mathrm{~Hz}, 1 \mathrm{H})$, $7.27(\mathrm{dd}, J=7.6,1.6 \mathrm{~Hz}, 1 \mathrm{H}), 7.12(\mathrm{td}, J=7.6,1.0 \mathrm{~Hz}, 1 \mathrm{H}), 6.93(\mathrm{dt}, J=7.9 \mathrm{~Hz}, 1 \mathrm{H}), 3.31(\mathrm{~s}$,

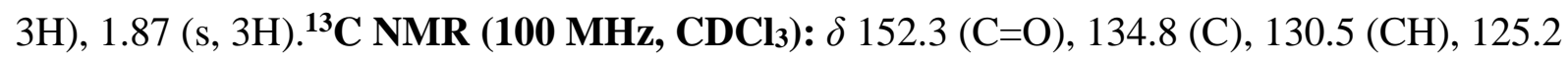
$(\mathrm{CH}), 123.8(\mathrm{CH}), 120.4(\mathrm{C}), 114.9(\mathrm{CH}), 107.0(\mathrm{C}), 51.1\left(\mathrm{CH}_{3}\right), 27.1\left(\mathrm{CH}_{3}\right)$. FT-IR (neat, $\mathbf{~ c m}^{-1}$ ): 3094, 2923, 2359, 2332, 1704, 1601, 1493, 1349, 1274, 1258. HRMS (ESI'): $m / z$ calcd. for $\mathrm{C}_{10} \mathrm{H}_{11} \mathrm{NO}_{3} \mathrm{Na}[\mathrm{M}+\mathrm{Na}]^{+} 216.0631$, found 216.0629 .

\section{4-Isopropyl-4-methoxy-1H-benzo[d][1,3]oxazin-2(4H)-one $4 \mathrm{j}$}<smiles>COC1(C(C)C)OC(=O)Nc2ccccc21</smiles>

General procedure A was followed with $\mathbf{3 j}(76.5 \mathrm{mg}, 0.40 \mathrm{mmol})$ and $\mathrm{MeOH}(2.5 \mathrm{~mL})$. Purification was performed with flash column chromatography over silica gel $\left(20 \mathrm{~g} \mathrm{SiO}_{2}\right.$, heptane/EtOAc, $100 / 0$ to $50 / 50$, gradient) to afford $\mathbf{4 j}$ as a light orange solid $(52.0 \mathrm{mg}$, 0.25 mmol, 59\%). ${ }^{1} \mathbf{H}$ NMR (600 MHz, $\left.\mathbf{C D C l}_{3}\right): \delta 9.32$ (brs, $\left.1 \mathrm{H}\right), 7.31(\mathrm{td}, J=7.8,1.4 \mathrm{~Hz}$, $1 \mathrm{H}), 7.20(\mathrm{dd}, J=7.0,0.7 \mathrm{~Hz}, 1 \mathrm{H}), 7.10(\mathrm{td}, J=7.6,1.0 \mathrm{~Hz}, 1 \mathrm{H}), 6.91(\mathrm{dd}, J=8.0,0.7 \mathrm{~Hz}$, 1H), $3.21(\mathrm{~s}, 3 \mathrm{H}), 2.27(\mathrm{~m}, 1 \mathrm{H}), 1.08(\mathrm{~d}, J=6.8 \mathrm{~Hz}, 3 \mathrm{H}), 0.87(\mathrm{~d}, J=6.8 \mathrm{~Hz}, 3 \mathrm{H}) .{ }^{13} \mathbf{C} \mathbf{~ N M R}$ (150 MHz, CDCl $): \delta 151.3(\mathrm{C}=\mathrm{O}), 138.1(\mathrm{C}), 129.4(\mathrm{CH}), 125.1(\mathrm{CH}), 122.5(\mathrm{CH}), 116.9$ (C), $113.5(\mathrm{CH}), 111.0(\mathrm{C}), 50.6\left(\mathrm{CH}_{3}\right), 38.9(\mathrm{CH}), 15.7\left(\mathrm{CH}_{3}\right), 14.5\left(\mathrm{CH}_{3}\right)$. FT-IR (neat, cm 1): 1703, 1601, 1494, 1461, 1434, 1373, 1332, 1271, 1252, 1248, 1143, 1132, 1096, 1042, 1012. HRMS (ESI $\left.{ }^{+}\right): m / z$ calcd. for $\mathrm{C}_{12} \mathrm{H}_{15} \mathrm{NO}_{3} \mathrm{Na}[\mathrm{M}+\mathrm{Na}]^{+} 244.0944$, found 244.0947.

\section{4-Cyclopentyl-4-methoxy-1H-benzo[d][1,3]oxazin-2(4H)-one $4 \mathrm{k}$}<smiles>CC1(C2CCCC2)OC(=O)Nc2ccccc21</smiles>

$4 \mathbf{k}$ 
General procedure A was followed with 3k $(86.9 \mathrm{mg}, 0.40 \mathrm{mmol})$ and $\mathrm{MeOH}(2.5 \mathrm{~mL})$. Purification was performed with flash column chromatography over silica gel $\left(20 \mathrm{~g} \mathrm{SiO}_{2}\right.$, heptane/EtOAc, $100 / 0$ to $50 / 50$, gradient) to afford $\mathbf{4 k}$ as a light orange solid $(35.1 \mathrm{mg}$, 0.14 mmol, 35\%). ${ }^{1} \mathbf{H}$ NMR (700 MHz, CDCl $): \delta 9.57$ (brs, $\left.1 \mathrm{H}\right), 7.29$ (td, $J=7.9,1.4 \mathrm{~Hz}$, $1 \mathrm{H}), 7.23(\mathrm{~d}, J=7.6 \mathrm{~Hz}, 1 \mathrm{H}), 7.09(\mathrm{td}, J=7.6,1.0 \mathrm{~Hz}, 1 \mathrm{H}), 6.93(\mathrm{dd}, J=8.0,0.6 \mathrm{~Hz}, 1 \mathrm{H})$, $3.21(\mathrm{~s}, 3 \mathrm{H}), 2.61-2.56(\mathrm{~m}, 1 \mathrm{H}), 1.83(\mathrm{dd}, J=12.9,8.4 \mathrm{~Hz}, 1 \mathrm{H}), 1.74-1.70(\mathrm{~m}, 1 \mathrm{H}), 1.66-1.63$ $(\mathrm{m}, 1 \mathrm{H}), 1.62-1.57(\mathrm{~m}, 1 \mathrm{H}), 1.55-1.44(\mathrm{~m}, 3 \mathrm{H}), 1.42-1.36(\mathrm{~m}, 1 \mathrm{H}) .{ }^{13} \mathrm{C}$ NMR $(\mathbf{1 7 6} \mathbf{~ M H z}$, $\left.\mathbf{C D C l}_{3}\right) \delta 152.5(\mathrm{C}=\mathrm{O}), 135.7(\mathrm{C}), 130.3(\mathrm{CH}), 126.0(\mathrm{CH}), 123.6(\mathrm{CH}), 118.8(\mathrm{C}), 114.7$ $(\mathrm{CH}), 111.4(\mathrm{C}), 51.5\left(\mathrm{CH}_{3}\right), 51.1\left(\mathrm{CH}_{3}\right), 27.2\left(\mathrm{CH}_{2}\right), 26.1\left(\mathrm{CH}_{2}\right), 25.9\left(\mathrm{CH}_{2}\right), 25.6\left(\mathrm{CH}_{2}\right)$. FT-IR (neat, $\left.\mathbf{c m}^{-1}\right)$ : 2922, 2359, 2333, 1703, 1602, 1493, 1346, 1247. HRMS (ESI ${ }^{+}$): $\mathrm{m} / \mathrm{z}$ calcd. for $\mathrm{C}_{14} \mathrm{H}_{17} \mathrm{NO}_{3} \mathrm{Na}[\mathrm{M}+\mathrm{Na}]^{+} 270.1101$, found 270.1100 .

\section{4-Allyl-4-methoxy-1H-benzo[d][1,3]oxazin-2(4H)-one 41}<smiles>C=CCC1(OC)OC(=O)Nc2ccccc21</smiles>

The general procedure A was followed with 31 (75.7 mg, $0.40 \mathrm{mmol})$ and $\mathrm{MeOH}(2.5 \mathrm{~mL})$. Purification was performed with flash column chromatography over silica gel $\left(20 \mathrm{~g} \mathrm{SiO}_{2}\right.$, heptane/EtOAc, 100/0 to 50/50, gradient) to afford $\mathbf{4 l}$ as a light yellow solid $(14.0 \mathrm{mg}$, 0.064 mmol, 16\%). ${ }^{1} \mathbf{H}$ NMR (700 MHz, CDCl $): \delta 9.47$ (brs, $1 \mathrm{H}$ ), 7.31 (ddd, $J=7.9,6.3$, $1.3 \mathrm{~Hz}, 1 \mathrm{H}), 7.24$ (d, $J=7.7 \mathrm{~Hz}, 1 \mathrm{H}), 7.13-7.08(\mathrm{~m}, 1 \mathrm{H}), 6.92(\mathrm{~d}, J=7.9 \mathrm{~Hz}, 1 \mathrm{H}), 5.66$ (ddt, $J$ $=17.3,10.2,7.2 \mathrm{~Hz}, 1 \mathrm{H}), 5.12-5.09(\mathrm{~m}, 1 \mathrm{H}), 5.09-5.07(\mathrm{~m}, 1 \mathrm{H}), 3.28(\mathrm{~s}, 3 \mathrm{H}), 2.87(\mathrm{dd}, J=$ 14.3, $7.5 \mathrm{~Hz}, 1 \mathrm{H}), 2.81(\mathrm{dd}, J=14.3,6.9 \mathrm{~Hz}, 1 \mathrm{H}) .{ }^{13} \mathbf{C}$ NMR (176 MHz, CDCl3): $\delta 151.7$ $(\mathrm{C}=\mathrm{O}), 135.4(\mathrm{C}), 130.4(\mathrm{CH}), 130.2(\mathrm{CH}), 125.7(\mathrm{CH}), 123.5(\mathrm{CH}), 120.4(\mathrm{C}), 118.1(\mathrm{C})$, $114.5(\mathrm{CH}), 108.5\left(\mathrm{CH}_{2}\right), 51.2(\mathrm{CH}), 45.9\left(\mathrm{CH}_{2}\right)$. FT-IR (neat, $\left.\mathbf{c m}^{-1}\right)$ : 3246, 3103, 2982, 2938, 1704, 1602, 1494, 1358, 1256, 1148. HRMS (ESI $\left.{ }^{+}\right): m / z$ calcd. for $\mathrm{C}_{12} \mathrm{H}_{13} \mathrm{NO}_{3} \mathrm{Na}$ $[\mathrm{M}+\mathrm{Na}]^{+} 242.0788$, found 242.0789 .

\section{4-Methoxy-6-methyl-4-phenyl-1H-benzo[d][1,3]oxazin-2(4H)-one 6a}<smiles>COC1(c2ccccc2)OC(=O)Nc2ccc(C)cc21</smiles>

The general procedure A was followed with 5a (95.7 $\mathrm{mg}, 0.40 \mathrm{mmol})$ and $\mathrm{MeOH}(2.5 \mathrm{~mL})$. Purification was performed with flash column chromatography over silica gel $\left(20 \mathrm{~g} \mathrm{SiO}_{2}\right.$, heptane/EtOAc, 100/0 to 50/50, gradient) to afford $\mathbf{6 a}$ as a white solid (35.7 $\mathrm{mg}, 0.13 \mathrm{mmol}$, 33\%). ${ }^{1}$ H NMR (400 MHz, CDCl 3 ): $\delta 9.08$ (brs, 1H), 7.52 (dd, $\left.J=7.7,1.6 \mathrm{~Hz}, 2 \mathrm{H}\right), 7.45$ $7.38(\mathrm{~m}, 3 \mathrm{H}), 7.11(\mathrm{~d}, J=8.2 \mathrm{~Hz}, 1 \mathrm{H}), 6.84(\mathrm{~d}, J=7.8 \mathrm{~Hz}, 2 \mathrm{H}), 3.43(\mathrm{~s}, 3 \mathrm{H}), 2.24(\mathrm{~s}, 3 \mathrm{H}) .{ }^{13} \mathbf{C}$ NMR (100 MHz, CDCl3): $\delta 151.7$ (C=O), 138.8 (C), 133.4 (C), 132.7 (C), 131.2 (CH), 129.3 
$(\mathrm{CH}), 128.6(2 \mathrm{CH}), 127.0(\mathrm{CH}), 126.9(2 \mathrm{CH}), 120.6(\mathrm{C}), 114.7(\mathrm{CH}), 107.5(\mathrm{C}), 51.9\left(\mathrm{CH}_{3}\right)$, $21.0\left(\mathrm{CH}_{3}\right)$. FT-IR (neat, $\left.\mathbf{c m}^{-1}\right)$ : 1708, 1607, 1512, 1450, 1347, 1251, 1226, 1195, 1164, 1095, 1075, 1008. HRMS (ESI $\left.{ }^{+}\right): m / z$ calcd. for $\mathrm{C}_{16} \mathrm{H}_{15} \mathrm{NO}_{3} \mathrm{Na}[\mathrm{M}+\mathrm{Na}]^{+} 292.0944$, found 292.0941 .

6-Bromo-4-methoxy-4-phenyl-1H-benzo[d][1,3]oxazin-2(4H)-one $6 \mathrm{~b}$<smiles>COC1(c2ccccc2)OC(=O)Nc2ccc(Br)cc21</smiles>

6b

The general procedure A was followed with $\mathbf{5 b}(122 \mathrm{mg}, 0.40 \mathrm{mmol})$ and $\mathrm{MeOH}(2.5 \mathrm{~mL})$. Purification was performed with flash column chromatography over silica gel $\left(20 \mathrm{~g} \mathrm{SiO}_{2}\right.$, heptane/EtOAc, 100/0 to 50/50, gradient) to afford $\mathbf{6 b}$ as a light yellow solid (60.9 $\mathrm{mg}, 0.18$ mmol, 46\%). ${ }^{1}$ H NMR (500 MHz, DMSO-d6): $\delta 10.85$ (brs, $1 \mathrm{H}$ ), 7.54 (dd, $J=8.6,2.2 \mathrm{~Hz}$, $1 \mathrm{H}), 7.52-7.42(\mathrm{~m}, 5 \mathrm{H}), 7.03(\mathrm{~d}, J=8.6 \mathrm{~Hz}, 1 \mathrm{H}), 6.95(\mathrm{~d}, J=2.2 \mathrm{~Hz}, 1 \mathrm{H}), 3.27(\mathrm{~s}, J=6.6 \mathrm{~Hz}$, 3H). ${ }^{13} \mathrm{C}$ NMR (126 MHz, DMSO-d6): $\delta 148.8(\mathrm{C}=\mathrm{O}), 138.5(\mathrm{C}), 135.2(\mathrm{C}), 133.3(\mathrm{CH})$, $129.3(\mathrm{CH}), 128.7(2 \mathrm{CH}), 128.5(\mathrm{CH}), 126.2(2 \mathrm{CH}), 122.4(\mathrm{C}), 116.8(\mathrm{CH}), 114.0(\mathrm{C}), 105.1$ (C), $51.1\left(\mathrm{CH}_{3}\right)$. HRMS $\left(\mathbf{E S I}^{+}\right): \mathrm{m} / z$ calcd. for $\mathrm{C}_{15} \mathrm{H}_{12} \mathrm{NO}_{3} \mathrm{BrNa}[\mathrm{M}+\mathrm{Na}]^{+}$355.9893, found 355.9886. The structure was analysed by X-Ray diffraction.

4,7-Dimethoxy-4-phenyl-1H-benzo[d][1,3]oxazin-2(4H)-one 6d<smiles>COc1ccc2c(c1)NC(=O)OC2(OC)c1ccccc1</smiles>

The general procedure A was followed with $5 \mathbf{d}(102 \mathrm{mg}, 0.40 \mathrm{mmol})$ and $\mathrm{MeOH}(2.5 \mathrm{~mL})$. Purification was performed with flash column chromatography over silica gel $\left(20 \mathrm{~g} \mathrm{SiO}_{2}\right.$, heptane/EtOAc, 100/0 to 50/50, gradient) to afford $\mathbf{6 d}$ as a light yellow solid (59.0 mg, $0.21 \mathrm{mmol}, 52 \%)$. ${ }^{\mathbf{1}} \mathbf{H}$ NMR (400 MHz, CDCl3): $\delta 9.85$ (brs, $\left.1 \mathrm{H}\right), 7.51$ (dd, $J=7.9,1.6 \mathrm{~Hz}$, 2H), 7.43-7.35 (m, 3H), $6.92(\mathrm{~d}, J=8.6 \mathrm{~Hz}, 1 \mathrm{H}), 6.57(\mathrm{dd}, J=8.6,2.4 \mathrm{~Hz}, 1 \mathrm{H}), 6.49(\mathrm{~d}, J=$ $2.3 \mathrm{~Hz}, 1 \mathrm{H}), 3.78$ (s, 3H), 3.42 (s, 3H). ${ }^{\mathbf{1 3}} \mathbf{C}$ NMR (100 MHz, CDCl $): \delta 161.4$ (C), 152.4 $(\mathrm{C}=\mathrm{O}), 139.3(\mathrm{C}), 136.5(\mathrm{C}), 129.2(\mathrm{CH}), 128.5(2 \mathrm{CH}), 128.2(\mathrm{CH}), 126.8(2 \mathrm{CH}), 112.7(\mathrm{C})$, $110.4(\mathrm{CH}), 107.8(\mathrm{C}), 99.5(\mathrm{CH}), 55.7\left(\mathrm{CH}_{3}\right), 51.8\left(\mathrm{CH}_{3}\right)$. FT-IR (neat, $\left.\mathbf{c m}^{-1}\right)$ : 3220, 3080, 2937, 2836, 1711, 1624, 1598, 1517, 1449, 1339, 1290, 1260. HRMS (ESI ${ }^{+}$): $\mathrm{m} / z$ calcd. for $\mathrm{C}_{16} \mathrm{H}_{15} \mathrm{NO}_{4} \mathrm{Na}[\mathrm{M}+\mathrm{Na}]^{+}$308.0893, found 308.0891 
<smiles>COc1cccc2c1NC(=O)OC2(C)c1ccccc1</smiles>

The general procedure $\mathbf{E}$ was followed with 5 e $(95.7 \mathrm{mg}, 0.40 \mathrm{mmol})$ and $\mathrm{MeOH}(2.5 \mathrm{~mL})$. Purification was performed with flash column chromatography over silica gel $\left(20 \mathrm{~g} \mathrm{SiO}_{2}\right.$, heptane/EtOAc, 100/0 to 50/50, gradient) to afford 6e as a light yellow solid $(32.3 \mathrm{mg}$, $0.12 \mathrm{mmol}, 30 \%)$. ${ }^{\mathbf{1}} \mathbf{H}$ NMR (600 MHz, $\left.\mathbf{C D C l}_{3}\right): \delta 8.32$ (brs, $\left.1 \mathrm{H}\right), 7.52$ (dd, $J=8.0,1.2 \mathrm{~Hz}$, $2 \mathrm{H}), 7.43-7.37(\mathrm{~m}, 3 \mathrm{H}), 7.16(\mathrm{~d}, J=7.3 \mathrm{~Hz}, 1 \mathrm{H}), 6.94(\mathrm{t}, J=7.6 \mathrm{~Hz}, 1 \mathrm{H}), 6.89$ (d, $J=7.6 \mathrm{~Hz}$, 1H), 3.44 (s, 3H), 2.34 (s, 3H). ${ }^{13} \mathbf{C}$ NMR (150 MHz, CDCl 3$): \delta 151.1(\mathrm{C}=\mathrm{O}), 138.6(\mathrm{C})$, $133.4(\mathrm{C}), 131.8(\mathrm{CH}), 129.2(\mathrm{CH}), 128.5(2 \mathrm{CH}), 127.0(2 \mathrm{CH}), 124.7(\mathrm{CH}), 123.1(\mathrm{CH})$, $122.8(\mathrm{C}), 121.0(\mathrm{C}), 107.2(\mathrm{C}), 52.0\left(\mathrm{CH}_{3}\right), 16.8\left(\mathrm{CH}_{3}\right)$. FT-IR (neat, $\left.\mathbf{c m}^{-1}\right)$ : 1706, 1600 , 1493, 1476, 1250, 1348, 1256, 1221, 1082, 1067, 1010. HRMS (ESI $\left.{ }^{+}\right): ~ m / z$ calcd. for $\mathrm{C}_{16} \mathrm{H}_{15} \mathrm{NO}_{3} \mathrm{Na}[\mathrm{M}+\mathrm{Na}]^{+} 292.0944$, found 292.0941 .

\section{4-Methoxy-4-phenyl-8-(trifluoromethyl)-1H-benzo[d][1,3]oxazin-2(4H)-one $6 f$}<smiles>COC1(c2ccccc2)OC(=O)Nc2c(C(F)(F)F)cccc21</smiles>

The general procedure $\mathbf{E}$ was followed with $\mathbf{5 f}(117 \mathrm{mg}, 0.40 \mathrm{mmol})$ and $\mathrm{MeOH}(2.5 \mathrm{~mL})$. Purification was performed with flash column chromatography over silica gel $\left(20 \mathrm{~g} \mathrm{SiO}_{2}\right.$, heptane/EtOAc, 100/0 to 50/50, gradient) to afford 6f as a light yellow solid $(74.7 \mathrm{mg}$, 0.23 mmol, 58\%). ${ }^{\mathbf{1}} \mathbf{H}$ NMR (700 MHz, CDCl3): $\delta 7.64$ (brs, $\left.1 \mathrm{H}\right), 7.60(\mathrm{~d}, J=7.8 \mathrm{~Hz}, 1 \mathrm{H})$, 7.54-7.50 (m, 2H), 7.47-7.42 (m, 3H), $7.24(\mathrm{~d}, J=7.8 \mathrm{~Hz}, 1 \mathrm{H}), 7.11(\mathrm{t}, J=7.8 \mathrm{~Hz}, 1 \mathrm{H}), 3.44$ (s, 3H). ${ }^{13} \mathbf{C}$ NMR (176 MHz, CDCl $): \delta 149.1(\mathrm{C}=\mathrm{O}), 137.0(\mathrm{C}), 132.9$ (q, $\left.J^{\mathrm{CF}}=1.3 \mathrm{~Hz}, \mathrm{C}\right)$, $131.0(\mathrm{CH}), 129.7(\mathrm{CH}), 128.9(2 \mathrm{CH}), 127.7\left(\mathrm{q}, J^{\mathrm{CF}}=4.8 \mathrm{~Hz}, \mathrm{CH}\right), 127.1(2 \mathrm{CH}), 123.3(\mathrm{q}$, $\left.J^{\mathrm{CF}}=272.9 \mathrm{~Hz}, \mathrm{C}\right), 123.7(\mathrm{C}), 123.0(\mathrm{CH}), 115.1\left(\mathrm{q}, J^{\mathrm{CF}}=32.8 \mathrm{~Hz}, \mathrm{C}\right), 106.3(\mathrm{C}), 52.1\left(\mathrm{CH}_{3}\right)$. ${ }^{19}$ F NMR (376 MHz, CDCl3): $\delta$-60.3. FT-IR (neat, cm$^{-1}$ ): 3264, 3218, 3157, 2359, 2332, 1732, 1602, 1452, 1324, 1275, 1266, 1167, 1117. HRMS $\left(\mathbf{E S I}^{+}\right): \mathrm{m} / z$ calcd. for $\mathrm{C}_{16} \mathrm{H}_{12} \mathrm{~F}_{3} \mathrm{NO}_{3} \mathrm{Na}[\mathrm{M}+\mathrm{Na}]^{+}$346.0661, found 346.0663. 
<smiles>CN1C(=O)Nc2ccccc2C1(O)c1ccccc1</smiles>

The general procedure A was followed with 1 (90.1 mg, $0.40 \mathrm{mmol})$ and $\mathrm{MeNH}_{2}(1.0 \mathrm{M}$ in THF, $4.0 \mathrm{~mL}, 20$ equiv.) in $1.0 \mathrm{~mL}$ of THF. Purification was performed with flash column chromatography over silica gel (20 g SiO 2 , heptane/EtOAc, 100/0 to 50/50, gradient) to afford 10 as a yellow solid (64.7 mg, $0.25 \mathrm{mmol}, 64 \%$ ). ${ }^{1} \mathbf{H}$ NMR (400 MHz, DMSO-d6): $\delta 9.73$ (brs, 1H), 7.42-7.38 (m, 2H), 7.37-7.32 (m, 2H), 7.25 (dd, $J=7.3,6.5 \mathrm{~Hz}, 1 \mathrm{H}), 7.11$ (ddd, $J=$ 7.7, 7.7, $1.4 \mathrm{~Hz}, 1 \mathrm{H}), 7.00(\mathrm{~s}, 1 \mathrm{H}), 6.88(\mathrm{dd}, J=7.7,1.5 \mathrm{~Hz}, 1 \mathrm{H}), 6.82$ (dd, $J=7.7,1.4 \mathrm{~Hz}$, 1H), 6.77 (ddd, $J=7.4,7.3,1.4 \mathrm{~Hz}, 1 \mathrm{H}), 2.60$ (s, 3H). ${ }^{13}$ C NMR (100 MHz, DMSO-d6): $\delta$ $151.6(\mathrm{C}=\mathrm{O}), 145.3(\mathrm{C}), 134.8(\mathrm{C}), 128.5(\mathrm{CH}), 128.2(2 \mathrm{CH}), 128.0(\mathrm{CH}), 127.4(\mathrm{CH}), 125.7$ $(2 \mathrm{CH}), 124.4(\mathrm{C}), 120.9(\mathrm{CH}), 113.3(\mathrm{CH}), 86.8(\mathrm{C}), 28.0\left(\mathrm{CH}_{3}\right)$. FT-IR (neat, $\left.\mathbf{c m}^{-1}\right)$ : 1651, $1605,1513,1499,1469,1445,1431,1398,1347,1327,1278,1186,1168,1023$. HRMS (ESI ${ }^{+}$): $m / z$ calcd. for $\mathrm{C}_{15} \mathrm{H}_{14} \mathrm{~N}_{2} \mathrm{O}_{2}[\mathrm{M}-\mathrm{OH}]^{+}$237.1022, found 237.1024 .

\section{Mechanistic Experiments (Scheme 3)}

\section{$5.1 \quad{ }^{18}$ O labelled experiments}

Synthesis of $1{ }^{18} \mathrm{O}$

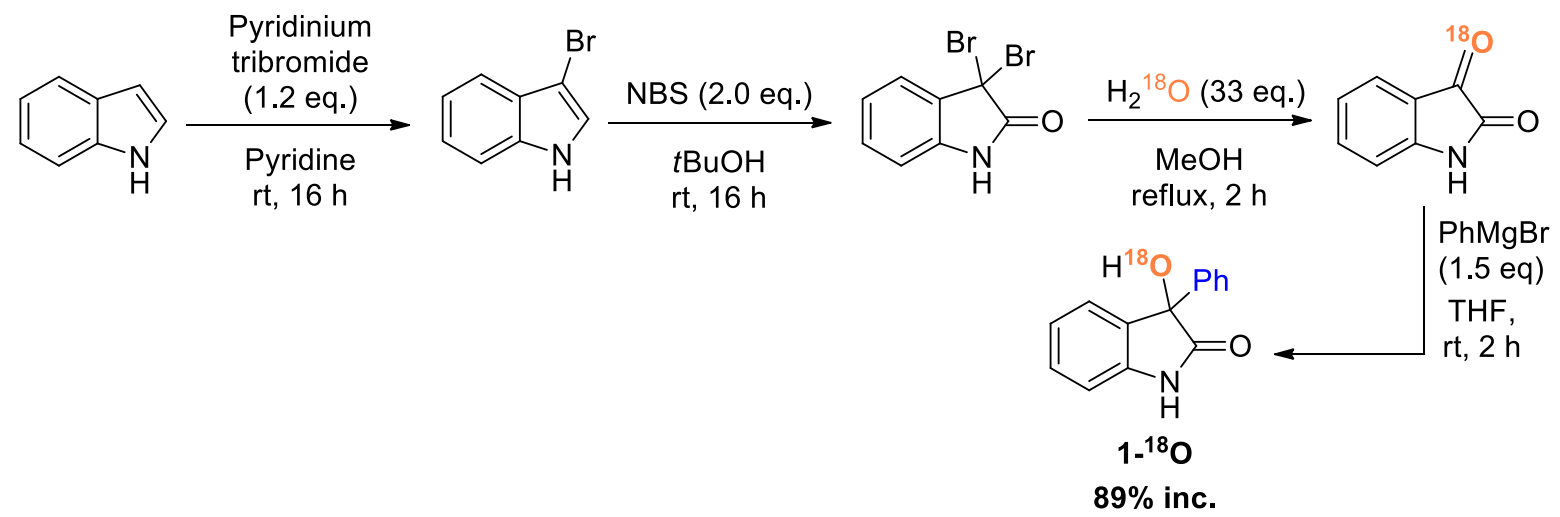

\section{3-Bromo-1H-indole}

Following a reported procedure, ${ }^{8}$ a $100 \mathrm{~mL}$ round bottom flask was charged with indole $(2.0 \mathrm{~g}, 17.1 \mathrm{mmol}, 1.0$ equiv. $)$ in pyridine $(20 \mathrm{~mL})$. Then, pyridinium tribromide $(6.6 \mathrm{~g}$, $20.5 \mathrm{mmol}, 1.2$ equiv) in pyridine $(20 \mathrm{~mL})$ was added dropwise over $10 \mathrm{~min}$ after which the reaction was left to stir $16 \mathrm{~h}$ at $\mathrm{rt}$. Next, ice water was added, and the reaction mixture was extracted with $\mathrm{Et}_{2} \mathrm{O}(20 \mathrm{~mL})$. The organic layers were washed with $6 \mathrm{~N} \mathrm{HCl}(20 \mathrm{~mL})$ and saturated aq. $\mathrm{NaHCO}_{3}(20 \mathrm{~mL})$, dried with $\mathrm{MgSO}_{4}$, filtered and concentrated in vacuo. The 
desired product $(1.99 \mathrm{~g}, 10.2 \mathrm{mmol}, 60 \%)$ is used directly in the next step without further purification.

\section{3,3-Dibromoindolin-2-one}

Following a reported procedure, ${ }^{9}$ a $50 \mathrm{~mL}$ round bottom flask was charged with 3-bromo- $1 \mathrm{H}$ indole $(1.0 \mathrm{~g}, 5.1 \mathrm{mmol}, 1.0$ equiv. $)$ in $t \mathrm{BuOH}(10 \mathrm{~mL})$. Then, $N$-bromosuccinimide $(1.8 \mathrm{~g}$, $10.2 \mathrm{mmol}, 2.0$ equiv) was added portion wise and reaction was stirred at $\mathrm{rt}$ for $16 \mathrm{~h}$. Next, a saturated solution of $\mathrm{Na}_{2} \mathrm{~S}_{2} \mathrm{O}_{3}$ was added, and the reaction mixture was extracted with $\mathrm{Et}_{2} \mathrm{O}$ $(20 \mathrm{~mL})$. The organic layers were washed with brine $(20 \mathrm{~mL})$, dried with $\mathrm{MgSO}_{4}$, filtered and concentrated in vacuo. The desired product ( $1.6 \mathrm{~g}, 5.5 \mathrm{mmol}$, quant.) is used directly in the next step without further purification.

\section{${ }^{18} \mathrm{O}$-Labelled Isatin}

In a sealed tube, 3,3-dibromoindolin-2-one (1.6 g, $5.5 \mathrm{mmol}, 1.0$ equiv.) was introduced followed by $\mathrm{MeOH}(10 \mathrm{ml})$ and $\mathrm{H}_{2}{ }^{18} \mathrm{O}(3.3 \mathrm{ml}, 181 \mathrm{mmol}, 33$ equiv.). The mixture was heated at reflux for $2 \mathrm{~h}$ and then concentrated in vacuo. Purification by $\mathrm{FC}\left(20 \mathrm{~g} \mathrm{SiO}_{2}\right.$, heptane/EA: 100/0 to 60/40) afforded the desired product as an orange solid (150 mg, 1.02 mmol, 19\%, 89\% incorporation). ${ }^{1} \mathbf{H}$ NMR (400 MHz, $\left.\mathbf{C D C l}_{3}\right): \delta 7.88$ (brs, 1H), 7.63 (d, $J=$ $7.6 \mathrm{~Hz}, 1 \mathrm{H}), 7.57(\mathrm{t}, J=7.9 \mathrm{~Hz}, 1 \mathrm{H}), 7.13(\mathrm{t}, J=7.6 \mathrm{~Hz}, 1 \mathrm{H}), 6.91(\mathrm{~d}, J=7.9 \mathrm{~Hz}, 1 \mathrm{H})$. HRMS (ESI $\left.{ }^{+}\right): m / z$ calcd. for $\mathrm{C}_{8} \mathrm{H}_{5} \mathrm{NO}^{18} \mathrm{ONa}[\mathrm{M}+\mathrm{Na}]^{+} 172.0255$, found 172.0254 .

\section{3-Hydroxy-3-phenylindolin-2-one $1 \mathbf{- 1}^{18} \mathrm{O}$}

General procedure B was followed with ${ }^{18} \mathrm{O}$-Labelled Isatin $(150 \mathrm{mg}, 1.02 \mathrm{mmol})$ and phenylmagnesium bromide ( $3.0 \mathrm{M}$ in $\mathrm{Et}_{2} \mathrm{O}, 0.68 \mathrm{~mL}, 2.04 \mathrm{mmol}, 2.0$ equiv.) to afford $\mathbf{1 -}^{18} \mathrm{O}$ as a yellow solid (90.4 mg, $0.40 \mathrm{mmol}, 39 \%) .{ }^{\mathbf{1}} \mathrm{H}$ NMR (400 MHz, DMSO-d6): $\delta 10.40$ (s, 1H), $7.33-7.23(\mathrm{~m}, 6 \mathrm{H}), 7.10(\mathrm{~d}, J=6.9 \mathrm{~Hz}, 1 \mathrm{H}), 6.96(\mathrm{td}, J=7.7,1.1 \mathrm{~Hz}, 1 \mathrm{H}), 6.91(\mathrm{~d}, J=7.7 \mathrm{~Hz}$, $1 \mathrm{H}), 6.63(\mathrm{~s}, 1 \mathrm{H}) . \mathbf{H R M S}\left(\mathbf{E S I}^{+}\right): \mathrm{m} / z$ calcd. for $\mathrm{C}_{14} \mathrm{H}_{11} \mathrm{NO}^{18} \mathrm{ONa}[\mathrm{M}+\mathrm{Na}]^{+} 250.0724$, found 250.0725 .

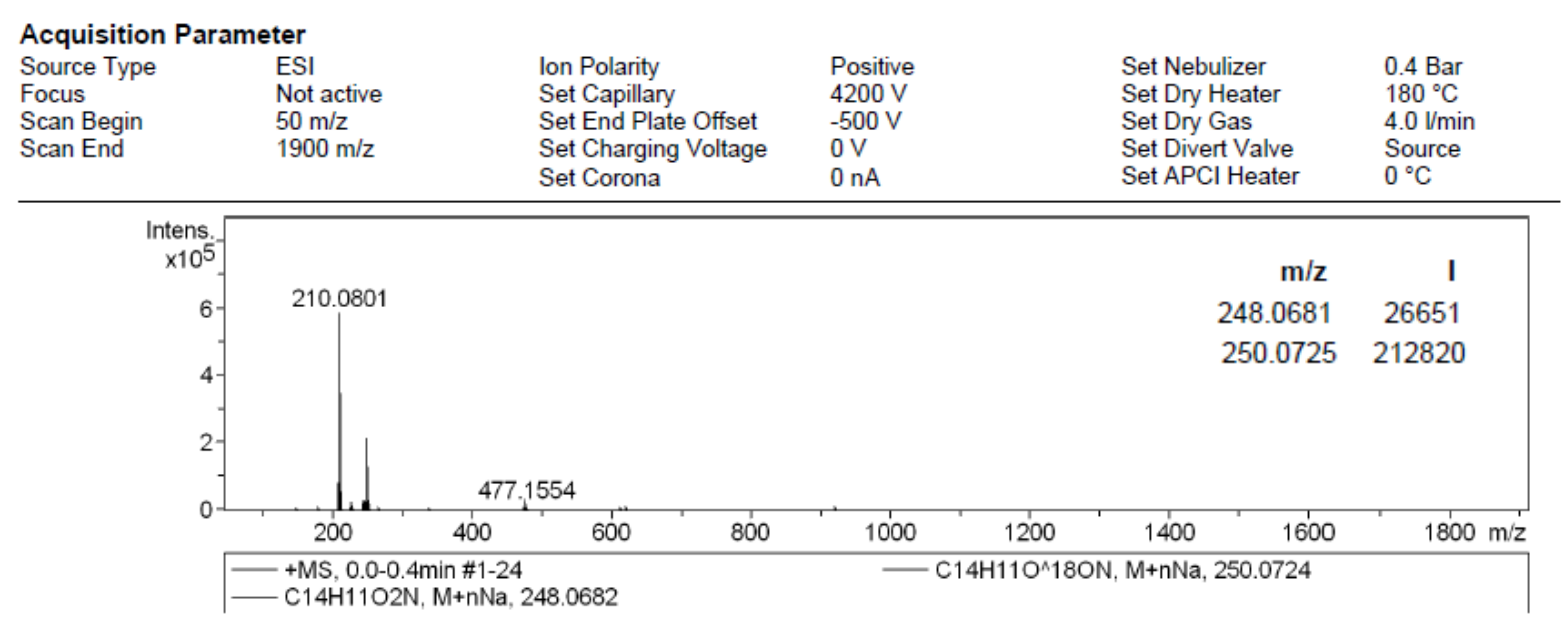


<smiles>CO[C@@]1(c2ccccc2)OC(=O)Nc2ccccc21</smiles>

General procedure A was followed with $\mathbf{1 - 1 8} \mathrm{O}(55.0 \mathrm{mg}, 0.38 \mathrm{mmol})$ and $\mathrm{MeOH}(2.5 \mathrm{~mL})$ Purification was performed with flash column chromatography over silica gel $\left(20 \mathrm{~g} \mathrm{SiO}_{2}\right.$, heptane/EtOAc, $100 / 0$ to 50/50, gradient) to afford $\mathbf{2 a - 1 8} \mathbf{O}$ as a white solid $(35.0 \mathrm{mg}$, $0.14 \mathrm{mmol}, 37 \%, 88 \%$ incorporation). ${ }^{1} \mathbf{H}$ NMR (400 MHz, CDCl $)$ ): $\delta 9.62$ (brs, 1H), 7.53 $(\mathrm{dd}, J=7.9,1.7 \mathrm{~Hz}, 2 \mathrm{H}), 7.45-7.35(\mathrm{~m}, 3 \mathrm{H}), 7.34-7.28(\mathrm{~m}, 1 \mathrm{H}), 7.08-7.00(\mathrm{~m}, 2 \mathrm{H}), 6.99(\mathrm{~d}, J$ $=8.0 \mathrm{~Hz}, 1 \mathrm{H}), 3.44(\mathrm{~s}, 3 \mathrm{H}) .{ }^{\mathbf{1 3}} \mathbf{C}$ NMR (100 MHz, CDCl$): \delta 153.0(\mathrm{C}=\mathrm{O}), 138.7(\mathrm{C}), 135.1$ $(\mathrm{C}), 130.6(\mathrm{CH}), 129.3(\mathrm{CH}), 128.6(2 \mathrm{CH}), 126.9(3 \mathrm{CH}), 123.7(\mathrm{CH}), 120.8(\mathrm{C}), 114.9(\mathrm{CH})$, $107.5(\mathrm{C}), 52.0\left(\mathrm{CH}_{3}\right)$. HRMS $\left(\mathbf{E S I}^{+}\right): \mathrm{m} / z$ calcd. for $\mathrm{C}_{15} \mathrm{H}_{13} \mathrm{NO}^{18} \mathrm{ONa}[\mathrm{M}+\mathrm{Na}]^{+} 280.0830$, found 280.0829 .

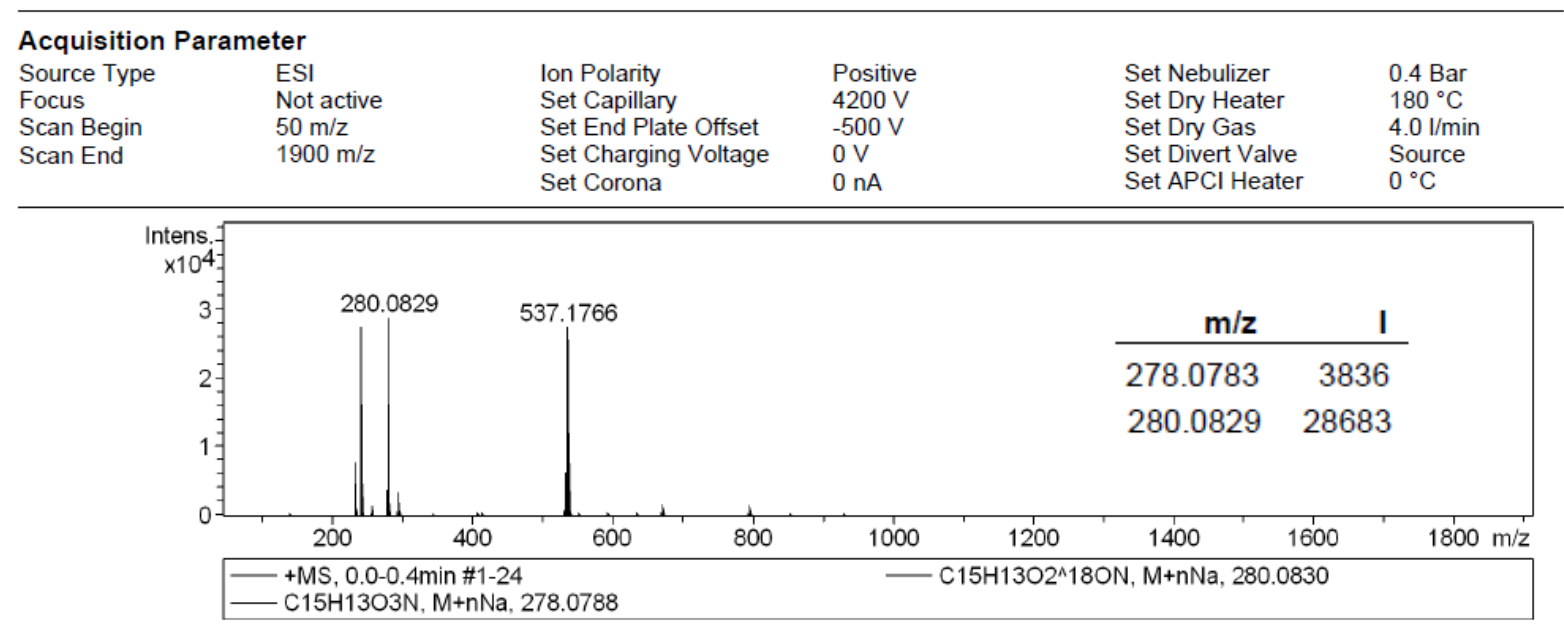

\subsection{Formation of methyl (2-benzoylphenyl)carbamate 7}

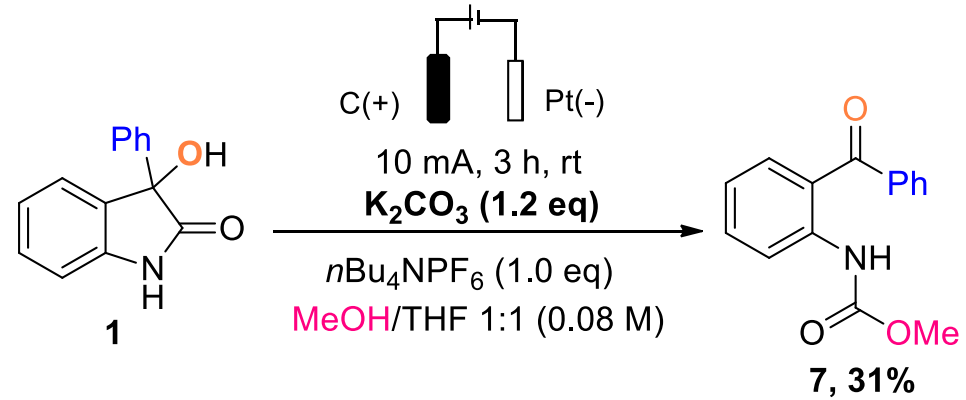

General procedure A was followed with $1(90.1 \mathrm{mg}, 0.40 \mathrm{mmol})$ and $\mathrm{MeOH}(2.5 \mathrm{~mL})$ in presence of $\mathrm{K}_{2} \mathrm{CO}_{3}$ (1.2 equiv.). Purification was performed with flash column chromatography over silica gel (20 $\mathrm{g} \mathrm{SiO}_{2}$, heptane/EtOAc, 100/0 to 50/50, gradient) to afford 


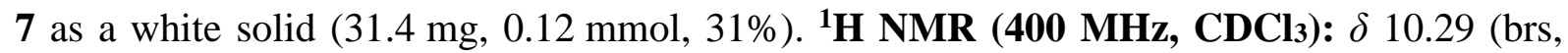
$1 \mathrm{H}), 8.42(\mathrm{~d}, J=8.4 \mathrm{~Hz}, 1 \mathrm{H}), 7.69(\mathrm{~d}, J=7.1 \mathrm{~Hz}, 2 \mathrm{H}), 7.61-7.50(\mathrm{~m}, 3 \mathrm{H}), 7.47$ (t, $J=7.6 \mathrm{~Hz}$, 2H), $7.03(\mathrm{t}, J=7.6 \mathrm{~Hz}, 1 \mathrm{H}), 3.79$ (s, 3H). ${ }^{13} \mathbf{C}$ NMR (100 MHz, CDCl3): $\delta 199.4(\mathrm{C}=\mathrm{O})$, $154.4(\mathrm{C}=\mathrm{O}), 141.1(\mathrm{C}), 138.9(\mathrm{C}), 134.4(\mathrm{CH}), 133.7(\mathrm{CH}), 132.4(\mathrm{CH}), 130.0(2 \mathrm{CH}), 128.4$ $(2 \mathrm{CH}), 123.0(\mathrm{C}), 121.3(\mathrm{CH}), 120.0(\mathrm{CH}), 52.5\left(\mathrm{CH}_{3}\right) . \mathbf{H R M S}\left(\mathbf{E S I}^{+}\right): \mathrm{m} / \mathrm{z}$ calcd. for $\mathrm{C}_{15} \mathrm{H}_{13} \mathrm{NO}_{3} \mathrm{Na}[\mathrm{M}+\mathrm{Na}]^{+} 278.0788$, found 278.0789 .

\subsection{Reaction of peroxide 8 in the standard conditions}

Synthesis of 3-(tert-butylperoxy)-3-(4-methoxyphenyl)indolin-2-one $\mathbf{8}$<smiles>COc1ccc(C2C(=O)Nc3ccccc32)cc1</smiles>

S8<smiles>CCCCOC1(c2ccc(OC)cc2)C(=O)Nc2ccccc21</smiles>

8

Following a reported procedure, ${ }^{10}$ a flamed-dried schlenk tube was charged with 3-(4methoxyphenyl)indolin-2-one S8 (300 mg, $1.25 \mathrm{mmol}, 1$ equiv) and copper(I) chloride ( $12.4 \mathrm{mg}, 0.13 \mathrm{mmol}, 0.1$ equiv). The tube is sealed, then evacuated and backfilled with argon (3 times). Then, $\mathrm{CH}_{2} \mathrm{Cl}_{2}(12 \mathrm{~mL})$ is added, followed by dropwise addition of tert-butyl hydroperoxide $(5.5 \mathrm{M}$ in decane, $2.5 \mathrm{~mL}, 2$ equiv) and the reaction is stirred at $\mathrm{rt}$ for $15 \mathrm{~h}$. The reaction mixture is dry-loaded onto silica gel and purified directly by FC (40 g $\mathrm{SiO}_{2}$, heptane/EtOAc, 100/0 to 50/50, gradient) to afford 8 (210 mg, $0.64 \mathrm{mmol}, 51 \%) .{ }^{1} \mathbf{H}$ NMR (700 MHz, CDCl $): \delta 8.36$ (brs, 1H), $7.40(\mathrm{~d}, J=9.0 \mathrm{~Hz}, 2 \mathrm{H}), 7.34(\mathrm{~d}, J=7.4 \mathrm{~Hz}, 1 \mathrm{H}), 7.30$ $(\mathrm{td}, J=7.7,1.2 \mathrm{~Hz}, 1 \mathrm{H}), 7.09(\mathrm{td}, J=7.6,0.9 \mathrm{~Hz}, 1 \mathrm{H}), 6.89(\mathrm{~d}, J=7.8 \mathrm{~Hz}, 1 \mathrm{H}), 6.86(\mathrm{~d}, J=$

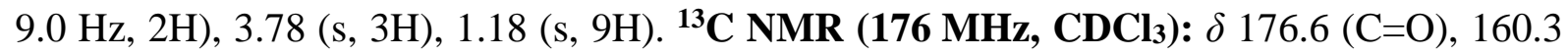
(C), $141.8(\mathrm{C}), 129.8(\mathrm{CH}), 128.9(\mathrm{C}), 128.9(2 \mathrm{CH}), 127.9(\mathrm{C}), 126.6(\mathrm{CH}), 122.6(\mathrm{CH})$, $114.0(2 \mathrm{CH}), 110.3(\mathrm{CH}), 86.2(\mathrm{C}), 80.9(\mathrm{C}), 55.4\left(\mathrm{CH}_{3}\right), 26.7\left(3 \mathrm{CH}_{3}\right)$. HRMS (ESI $\left.{ }^{+}\right)$: $m / z$ calcd. for $\mathrm{C}_{19} \mathrm{H}_{21} \mathrm{NO}_{4} \mathrm{Na}[\mathrm{M}+\mathrm{Na}]^{+} 350.1363$, found 350.1364 .<smiles>CCOC1(c2ccc(OC)cc2)C(=O)Nc2ccccc21</smiles>

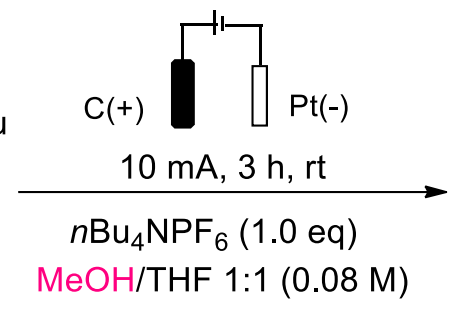<smiles>COc1ccc(C2(OC)OC(=O)Nc3ccccc32)cc1</smiles>

$4 a, 71 \%$

General procedure A was followed with $8(100 \mathrm{mg}, 0.31 \mathrm{mmol})$ and $\mathrm{MeOH}(2.5 \mathrm{~mL})$. Purification was performed with flash column chromatography over silica gel $\left(20 \mathrm{~g} \mathrm{SiO}_{2}\right.$, heptane/EtOAc, 100/0 to 50/50, gradient) to afford $\mathbf{4 a}$ as a white solid (62.0 $\mathrm{mg}, 0.22 \mathrm{mmol}$, 
71\%). ${ }^{1} \mathbf{H}$ NMR (400 MHz, CDCl $): \delta 8.79$ (brs, $\left.1 \mathrm{H}\right), 7.43$ (d, $\left.J=8.9 \mathrm{~Hz}, 2 \mathrm{H}\right), 7.31$ (ddd, $J=$ 8.0, 7.0, $1.9 \mathrm{~Hz}, 1 \mathrm{H}), 7.10-7.00(\mathrm{~m}, 2 \mathrm{H}), 6.94-6.90(\mathrm{~m}, 3 \mathrm{H}), 3.82(\mathrm{~s}, 3 \mathrm{H}), 3.43(\mathrm{~s}, 3 \mathrm{H}) .{ }^{13} \mathrm{C}$ NMR (100 MHz, CDCl $)$ : $\delta 160.3(\mathrm{C}=\mathrm{O}), 151.6(\mathrm{C}), 136.1(\mathrm{C}), 130.9(\mathrm{C}), 130.5(\mathrm{CH}), 128.4$ $(2 \mathrm{CH}), 126.9(\mathrm{CH}), 123.6(\mathrm{CH}), 121.3(\mathrm{C}), 114.7(\mathrm{CH}), 113.9(2 \mathrm{CH}), 107.6(\mathrm{C}), 55.5\left(\mathrm{CH}_{3}\right)$, $52.0\left(\mathrm{CH}_{3}\right)$. FT-IR (neat, $\left.\mathbf{c m}^{-1}\right)$ : 1700, 1604, 1513, 1505, 1494, 1432, 1353, 1319, 1305, 1251, 1209, 1185, 1169, 1153, 1095, 1032, 1009. HRMS $\left(\mathbf{E S I}^{+}\right): \mathrm{m} / z$ calcd. for $\mathrm{C}_{16} \mathrm{H}_{15} \mathrm{NO}_{4} \mathrm{Na}[\mathrm{M}+\mathrm{Na}]^{+}$308.0893, found 308.0887.

\subsection{BHT trapping experiment}

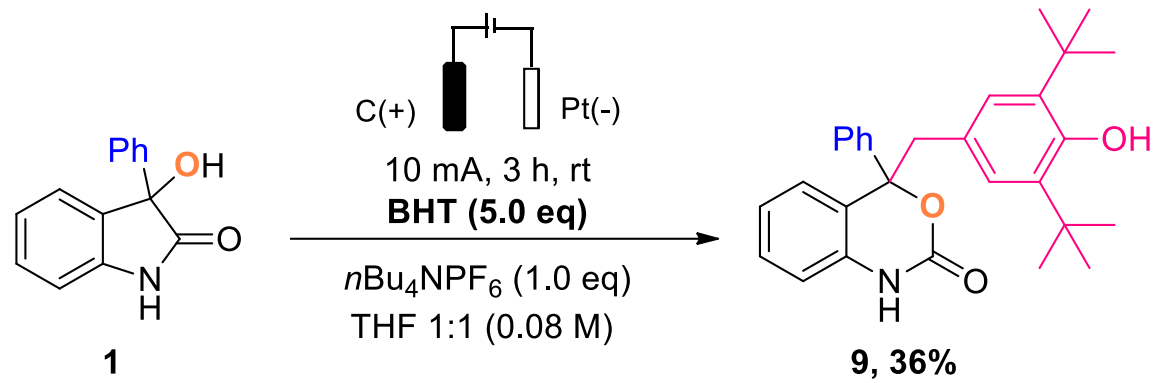

General procedure A was followed with $1(90.1 \mathrm{mg}, 0.40 \mathrm{mmol})$ and $\mathrm{MeOH}(2.5 \mathrm{~mL})$ in the presence of BHT (441 mg, $2.0 \mathrm{mmol}, 5.0$ equiv.). Purification was performed with flash column chromatography over silica gel ( $20 \mathrm{~g} \mathrm{SiO}_{2}$, heptane/EtOAc, 100/0 to 50/50, gradient) to afford $\mathbf{4 a}$ as a white solid $(66.2 \mathrm{mg}, 0.15 \mathrm{mmol}, 37 \%)$. 1a was also recovered $(52.8 \mathrm{mg}$,

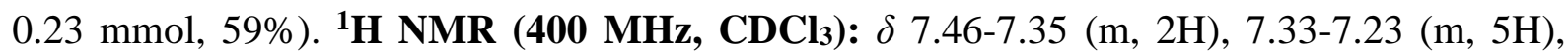
$7.11(\mathrm{~s}, 2 \mathrm{H}), 7.03(\mathrm{t}, J=7.5 \mathrm{~Hz}, 1 \mathrm{H}), 6.86(\mathrm{~d}, J=7.8 \mathrm{~Hz}, 1 \mathrm{H}), 5.17(\mathrm{~s}, 1 \mathrm{H}), 5.12$ (d, $J=15.4$ $\mathrm{Hz}, 1 \mathrm{H}), 4.55(\mathrm{~d}, J=15.4 \mathrm{~Hz}, 1 \mathrm{H}), 3.60(\mathrm{~s}, 1 \mathrm{H}), 1.39(\mathrm{~s}, 18 \mathrm{H}),{ }^{13} \mathbf{C}$ NMR (100 MHz, CDCl3): $\delta 177.8(\mathrm{C}=\mathrm{O}), 153.5(\mathrm{C}), 143.1(\mathrm{C}), 140.6(\mathrm{C}), 136.4(2 \mathrm{C}), 131.9(\mathrm{C}), 129.9(\mathrm{CH})$, $128.8(2 \mathrm{CH}), 128.4(\mathrm{CH}), 126.3(\mathrm{C}), 125.3(2 \mathrm{CH}), 125.0(\mathrm{CH}), 124.2(2 \mathrm{CH}), 123.5(\mathrm{CH})$, $109.9(\mathrm{CH}), 78.1(\mathrm{C}), 44.3\left(\mathrm{CH}_{2}\right), 34.4(2 \mathrm{C}), 30.4\left(6 \mathrm{CH}_{3}\right) . \mathbf{H R M S}\left(\mathbf{E S I}^{+}\right): \mathrm{m} / \mathrm{z}$ calcd. for $\mathrm{C}_{29} \mathrm{H}_{33} \mathrm{NO}_{3} \mathrm{Na}[\mathrm{M}+\mathrm{Na}]^{+} 466.2353$, found 466.2355 . 


\section{X-Ray Diffraction Analysis of compound $6 \mathrm{~b}$}

The X-ray intensity data was measured on Bruker D8 Venture diffractometer equipped with multilayer monochromator, Mo K/ $\alpha$ INCOATEC micro focus sealed tube and Oxford cooling system. The structure was solved by Charge Flipping. Non-hydrogen atoms were refined with anisotropic displacement parameters. Hydrogen atoms were inserted at calculated positions and refined with riding model. The following software was used: Bruker SAINT software package $^{11}$ using a narrow-frame algorithm for frame integration, $S A D A B S^{12}$ for absorption correction, $O L E X 2^{13}$ for structure solution, refinement, molecular diagrams and graphical user-interface, Shelxle ${ }^{14}$ for refinement and graphical user-interface SHELXS-2015 ${ }^{15}$ for structure solution, SHELXL-2015 $5^{15}$ for refinement, Platon ${ }^{16}$ for symmetry check. Experimental data and CCDC-Codes Experimental data (Available online: http://www.ccdc.cam.ac.uk/conts/retrieving.html) can be found in Table S1. Crystal data, data collection parameters, and structure refinement details are given in Table S2. Asymmetric Units visualized in Figure S1.

The sample was prepared by slow liquid-liquid diffusion using a mixture of $\mathrm{CH}_{2} \mathrm{Cl}_{2} / \mathrm{MeOH} / \mathrm{AcOEt}(1 / 1 / 1)$.

Table S1. Experimental parameter and CCDC-Code.

\begin{tabular}{|c|c|c|c|c|c|c|c|c|}
\hline Sample & Machine & Source & Temp. & $\begin{array}{c}\text { Detector } \\
\text { Distance }\end{array}$ & $\begin{array}{c}\text { Time/ } \\
\text { Frame }\end{array}$ & \#Frames & $\begin{array}{c}\text { Frame } \\
\text { width }\end{array}$ & CCDC \\
\hline & Bruker & & {$[\mathrm{K}]$} & {$[\mathrm{mm}]$} & {$[\mathrm{s}]$} & & {$\left[{ }^{\circ}\right]$} & \\
\hline 6b & D8 & Mo & 100 & 30 & 3 & 2299 & 0.360 & 2102358 \\
\hline
\end{tabular}

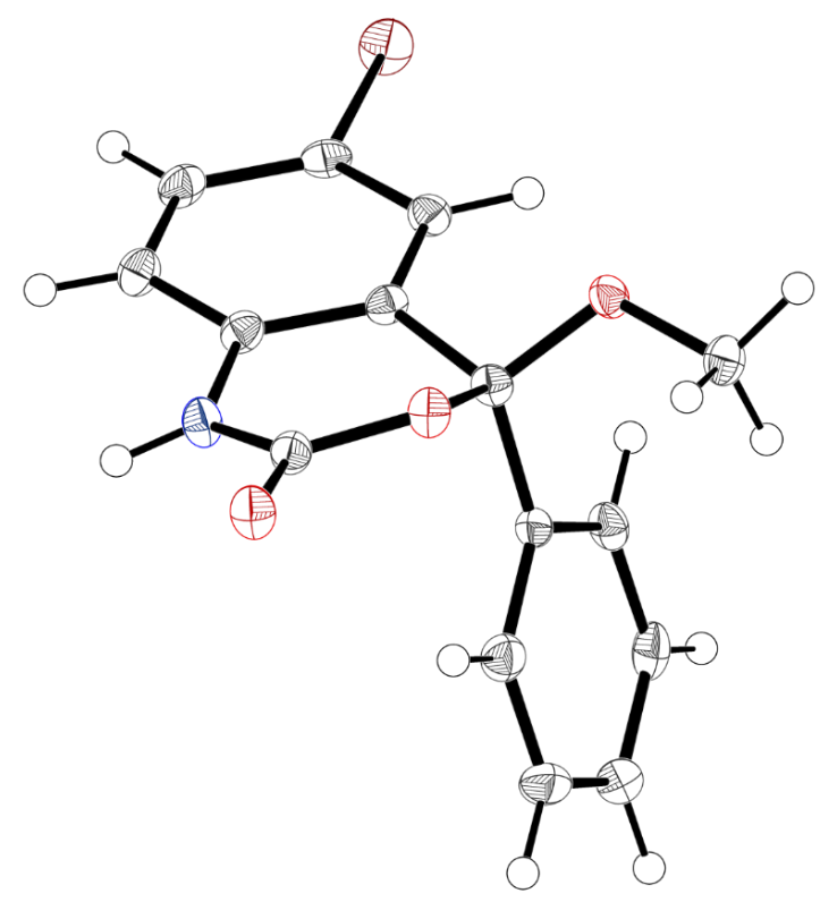

Figure S1. Crystal structure of $\mathbf{6 b}$ drawn with $50 \%$ displacement ellipsoid. Only one part visualized. The bond precision for C-C single bonds is $0.0057 \AA$. 


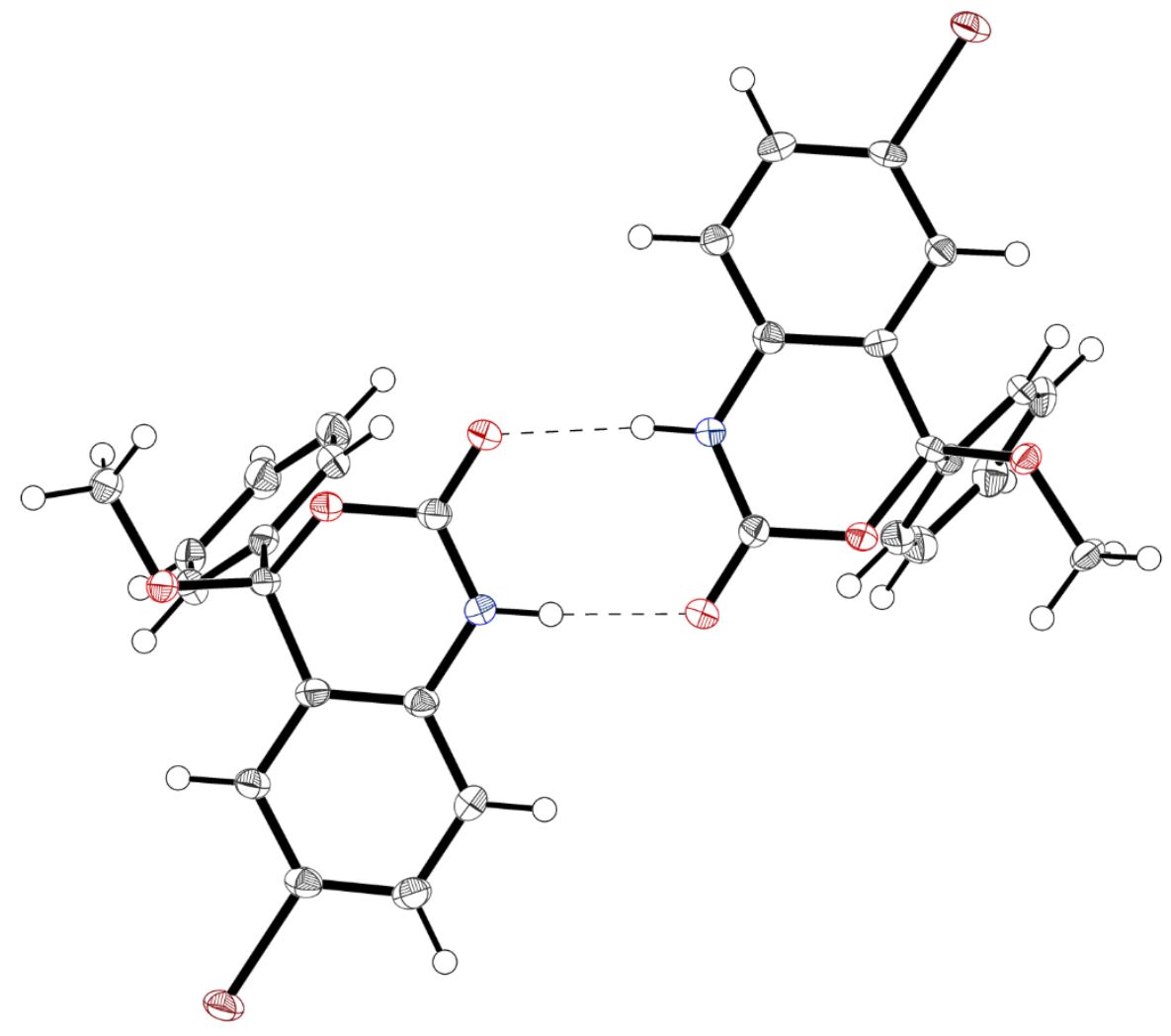

Figure S2. Crystal structure of $\mathbf{6 b}$ drawn with 50\% displacement ellipsoid. The bond precision for C-C single bonds is $0.0057 \AA$.

Table S2. Sample and crystal data, data collection and structure refinement. More detailed information can be found in the Cif Code of CCDC: 2102358.

\begin{tabular}{|c|c|}
\hline Identification code & mipa361_P-1 \\
\hline \hline Empirical formula & $\mathrm{C}_{15} \mathrm{H}_{12} \mathrm{BrNO}_{3}$ \\
\hline \hline Formula weight & 334.17 \\
\hline \hline Temperature/K & 100.0 \\
\hline \hline Crystal system & triclinic \\
\hline \hline Space group & $\mathrm{P}-1$ \\
\hline \hline $\mathrm{a} / \AA$ & $9.9912(6)$ \\
\hline \hline $\mathrm{b} / \AA$ & $11.1573(6)$ \\
\hline \hline $\mathrm{c} / \AA$ & $12.8256(7)$ \\
\hline \hline$\alpha /{ }^{\circ}$ & $99.968(2)$ \\
\hline \hline$\beta /{ }^{\circ}$ & $105.709(2)$ \\
\hline \hline$\gamma^{\circ}$ & $99.578(2)$ \\
\hline \hline
\end{tabular}




\begin{tabular}{|c|c|}
\hline Volume $/ \AA^{3}$ & $1320.82(13)$ \\
\hline $\mathrm{Z}$ & 4 \\
\hline$\rho_{\text {calc }} \mathrm{g} / \mathrm{cm}^{3}$ & 1.680 \\
\hline$\mu / \mathrm{mm}^{-1}$ & 3.118 \\
\hline $\mathrm{F}(000)$ & 672.0 \\
\hline Crystal size $/ \mathrm{mm}^{3}$ & $0.358 \times 0.313 \times 0.124$ \\
\hline Radiation & $\operatorname{MoK} \alpha(\lambda=0.71073)$ \\
\hline $2 \Theta$ range for data collection/ ${ }^{\circ}$ & 3.804 to 56.564 \\
\hline Index ranges & $-13 \leq \mathrm{h} \leq 12,-14 \leq \mathrm{k} \leq 14,0 \leq 1 \leq 17$ \\
\hline Reflections collected & 9148 \\
\hline Independent reflections & $6510\left[\mathrm{R}_{\mathrm{int}}=0.1797, \mathrm{R}_{\mathrm{sigma}}=0.0928\right]$ \\
\hline Data/restraints/parameters & $6510 / 0 / 363$ \\
\hline Goodness-of-fit on $\mathrm{F}^{2}$ & 1.067 \\
\hline Final $\mathrm{R}$ indexes $[\mathrm{I}>=2 \sigma(\mathrm{I})]$ & $\mathrm{R}_{1}=0.0763, \mathrm{wR}_{2}=0.1391$ \\
\hline Final $\mathrm{R}$ indexes [all data] & $\mathrm{R}_{1}=0.1015, \mathrm{wR}_{2}=0.1492$ \\
\hline Largest diff. peak/hole / e $\AA^{-3}$ & $2.29 /-1.28$ \\
\hline
\end{tabular}




\section{NMR Spectra}

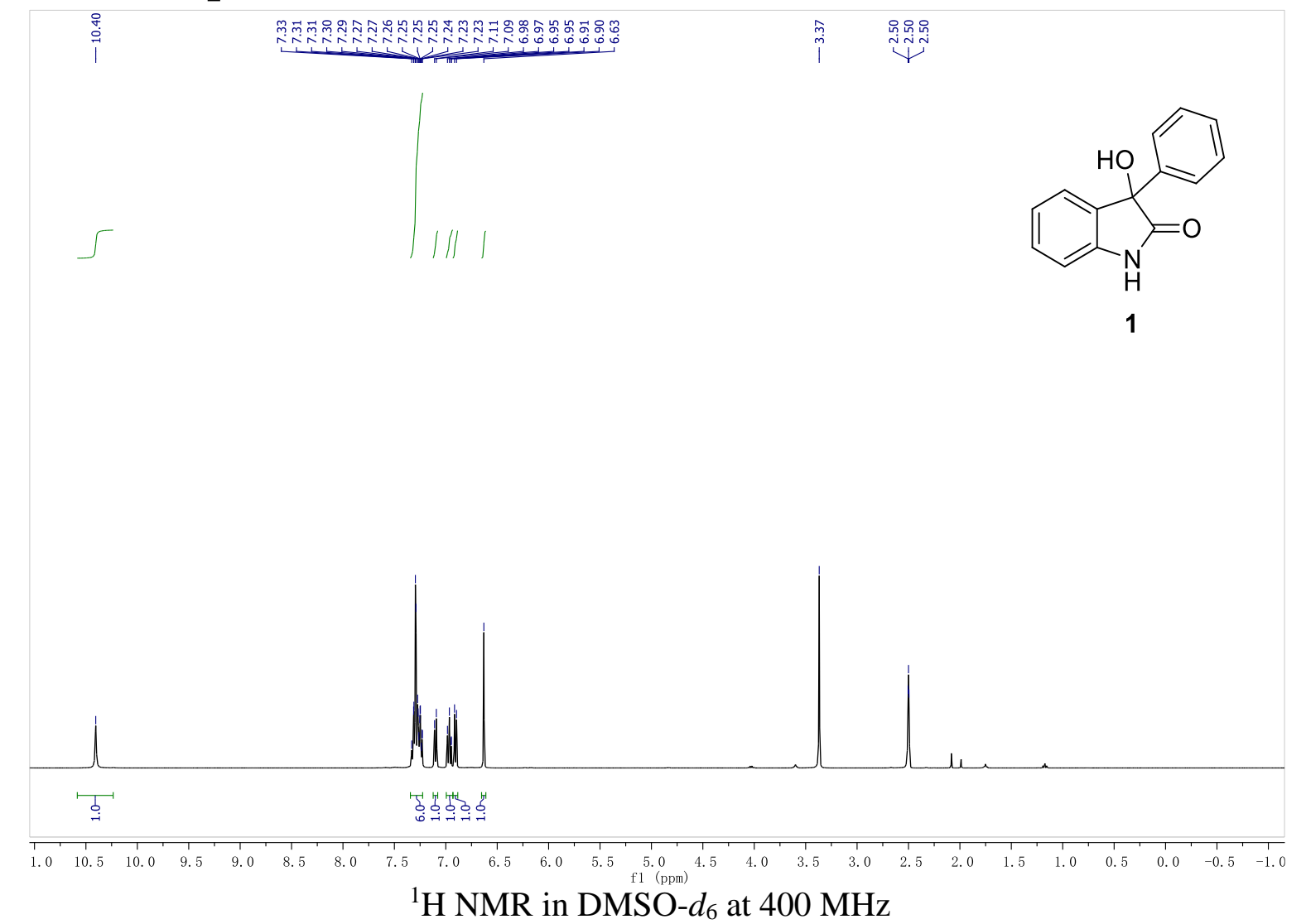

${ }^{1} \mathrm{H}$ NMR in DMSO- $d_{6}$ at $400 \mathrm{MHz}$

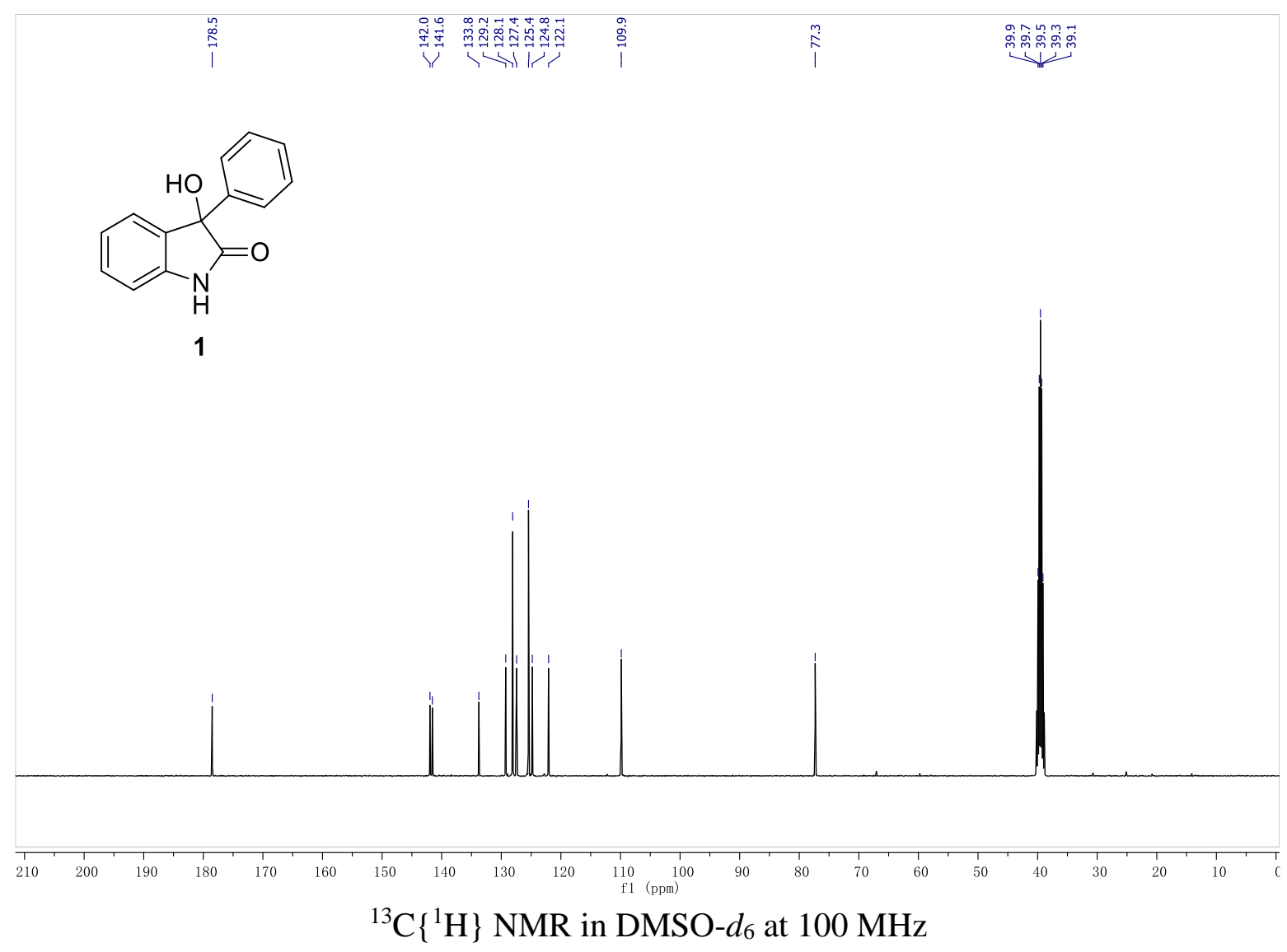




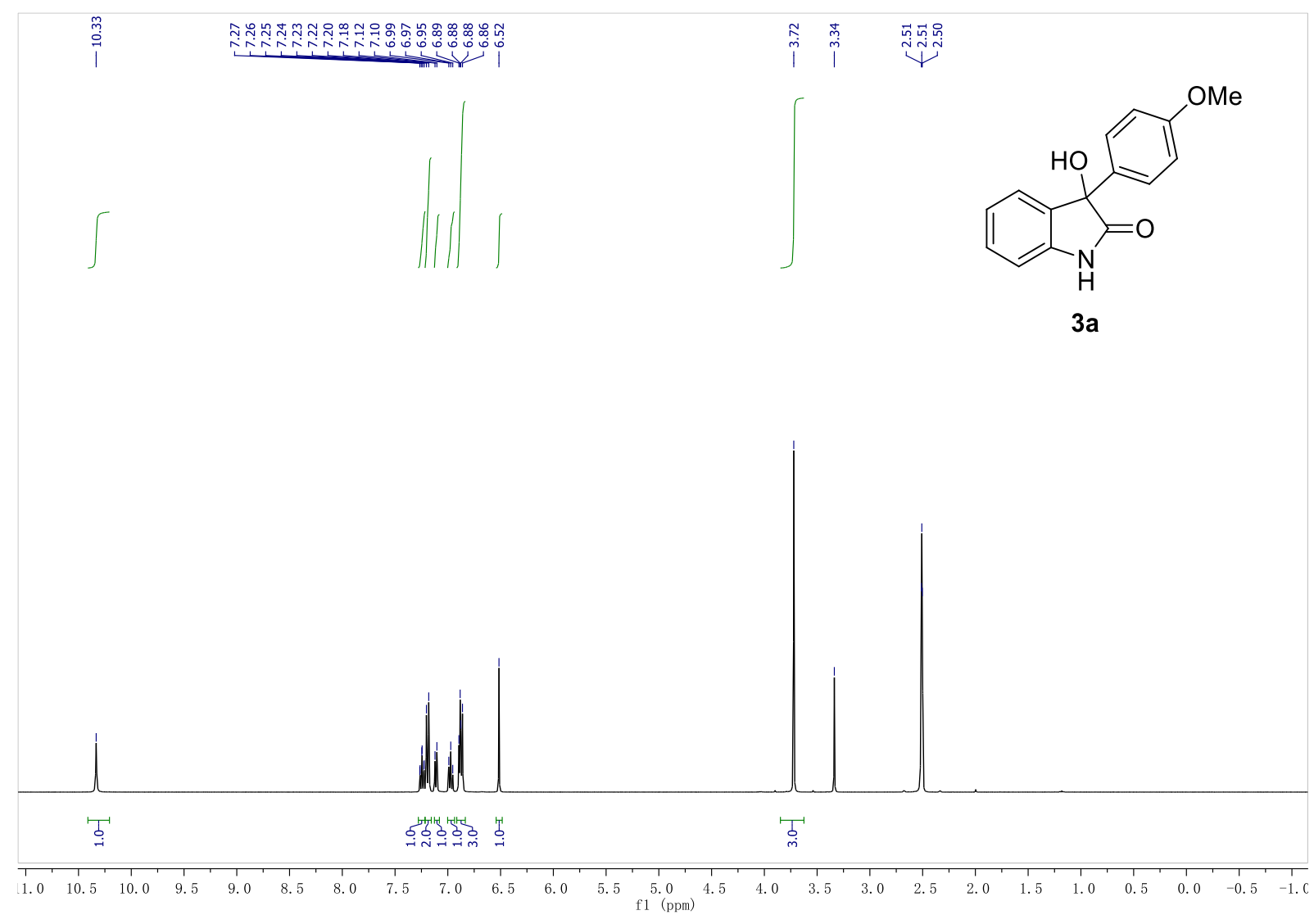

${ }^{1} \mathrm{H}$ NMR in DMSO- $d_{6}$ at $400 \mathrm{MHz}$

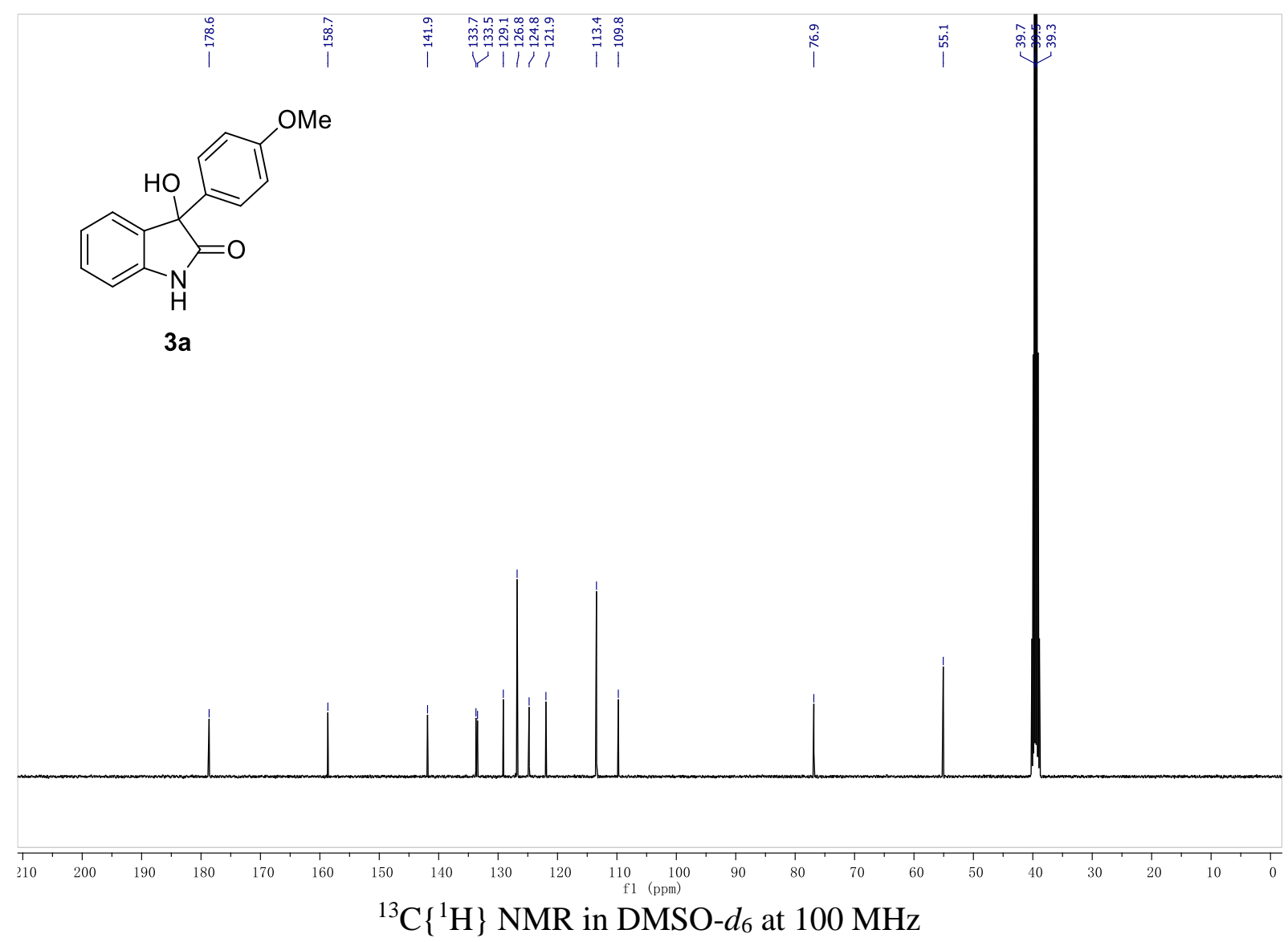




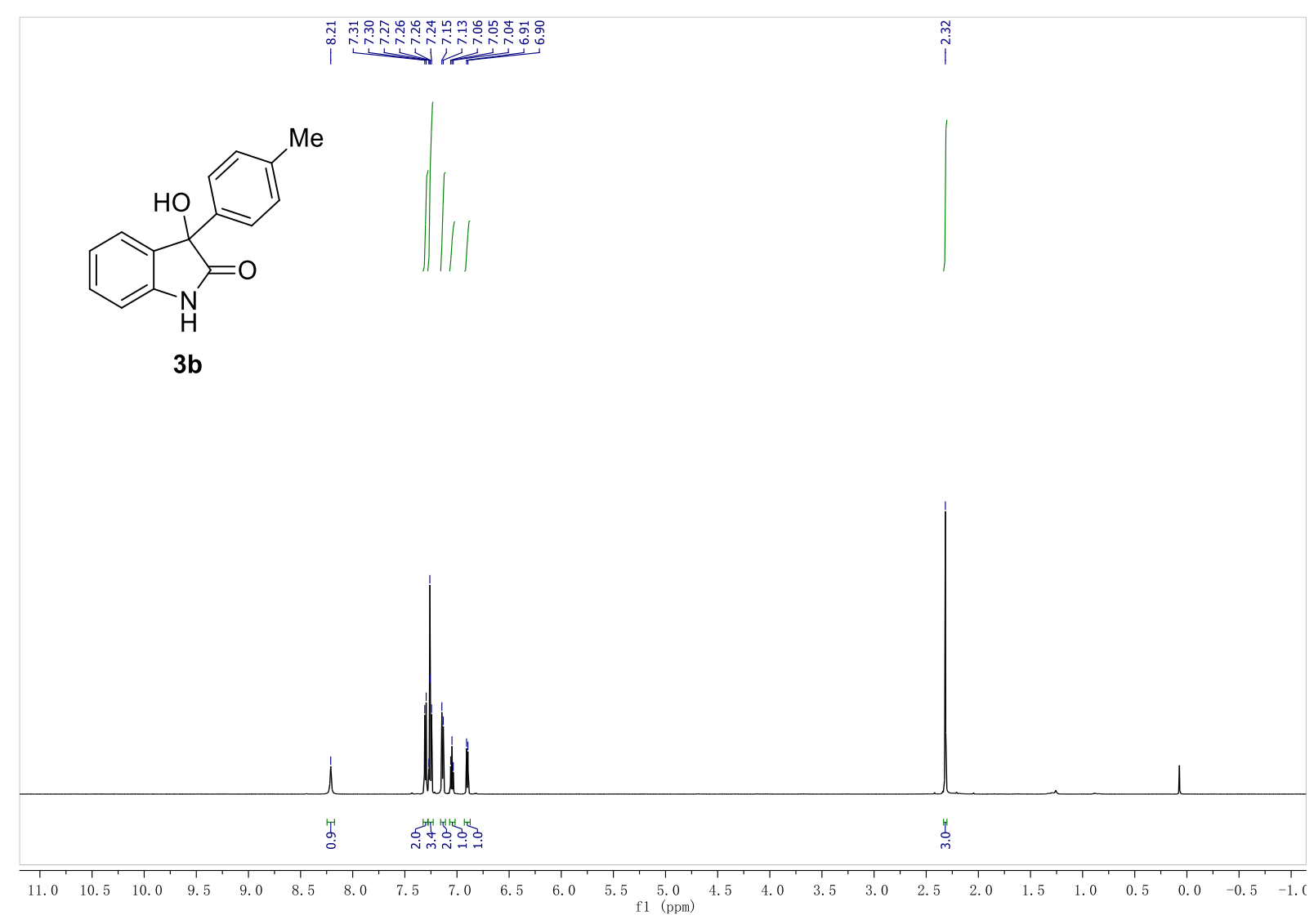

${ }^{1} \mathrm{H} \mathrm{NMR}$ in $\mathrm{CDCl}_{3}$ at $600 \mathrm{MHz}$

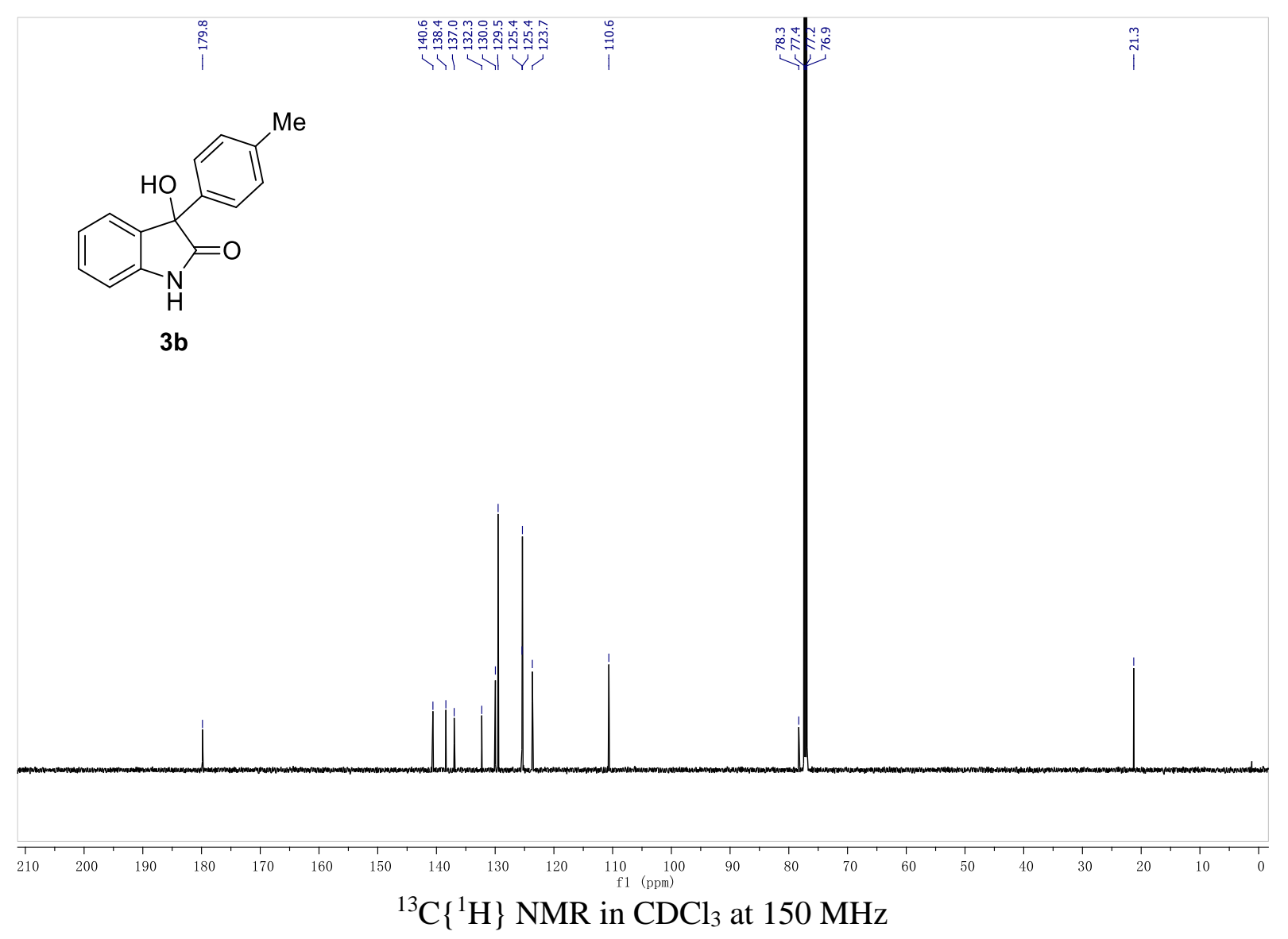




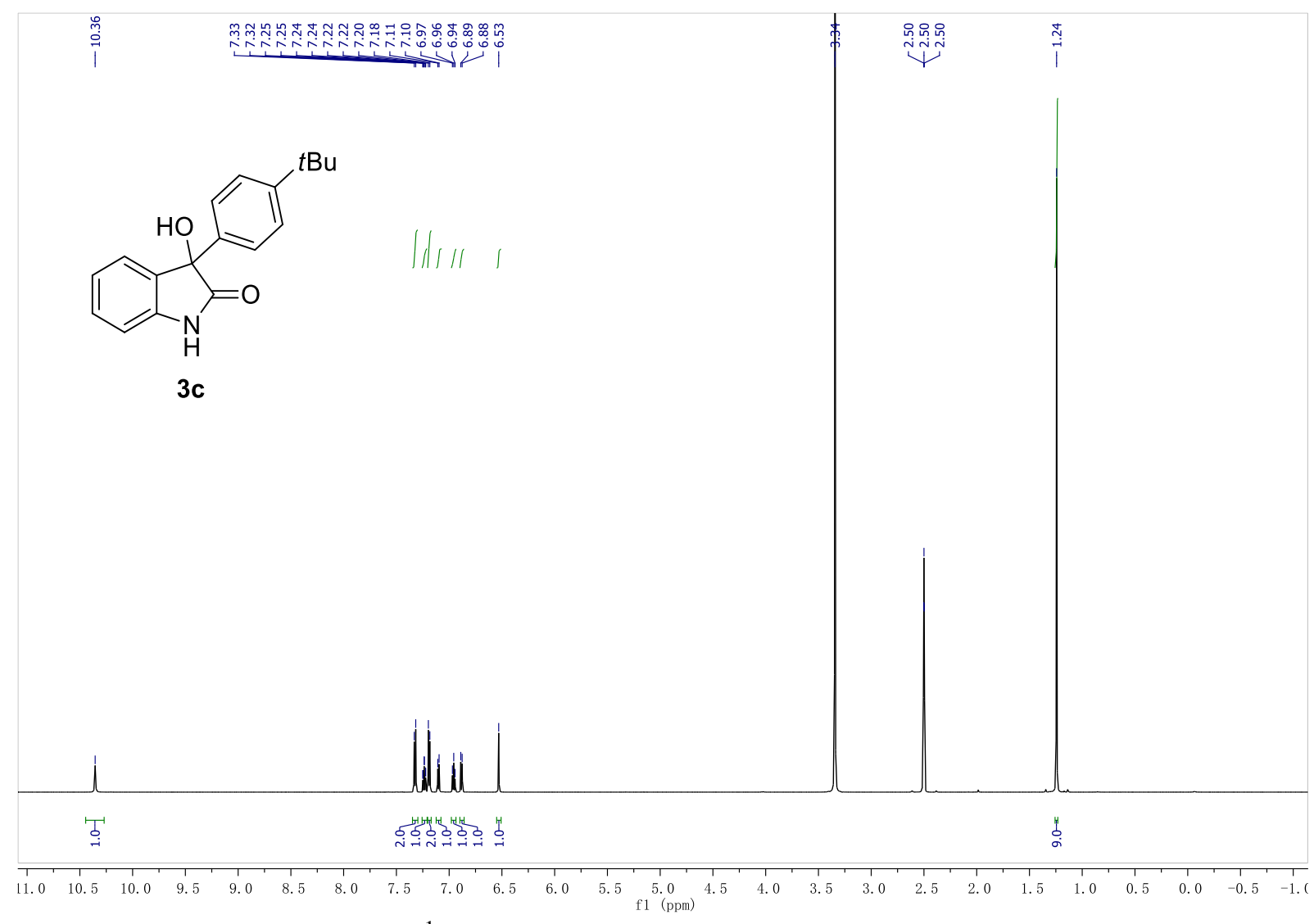

${ }^{1} \mathrm{H}$ NMR in DMSO- $d_{6}$ at $600 \mathrm{MHz}$

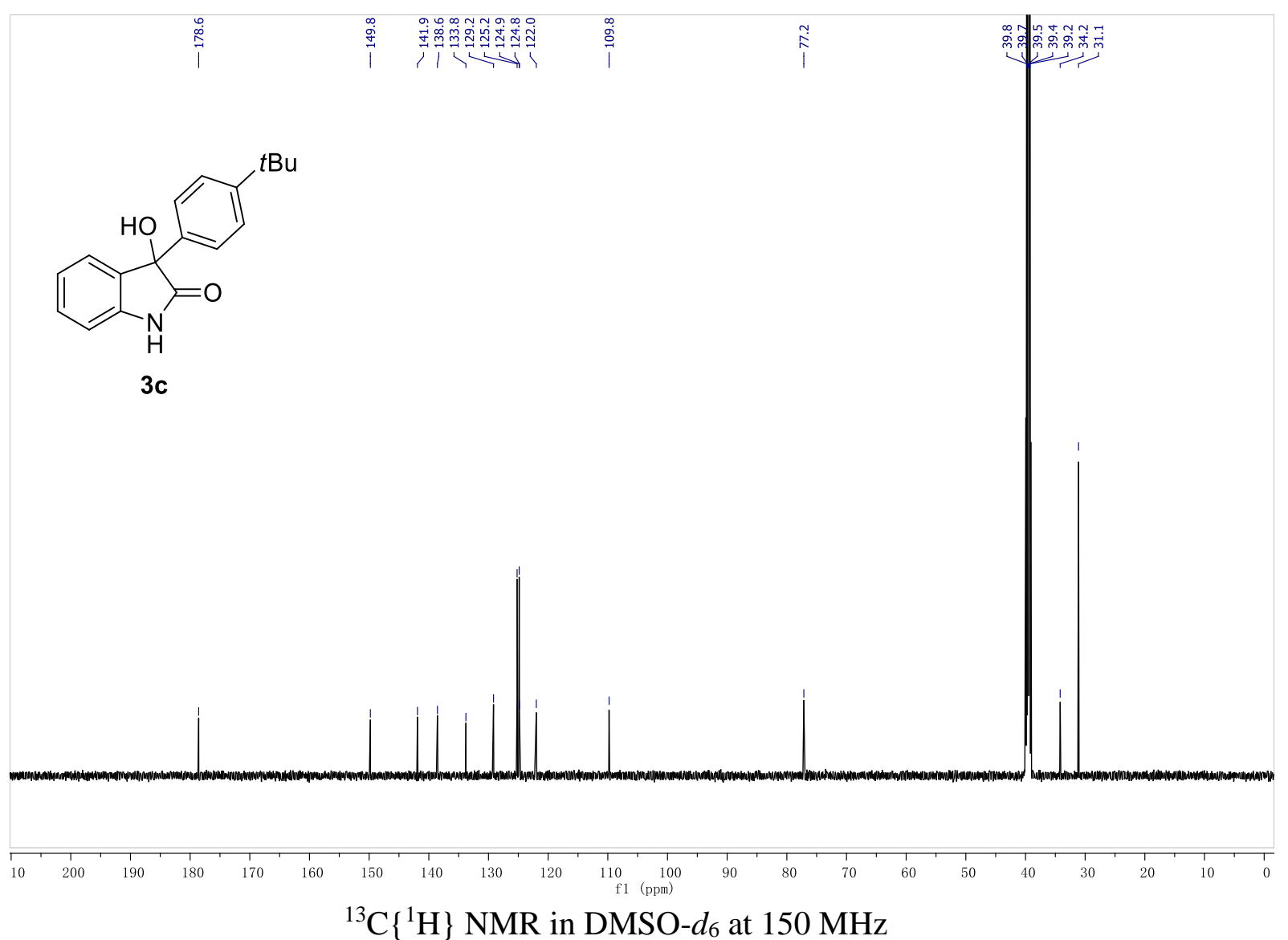




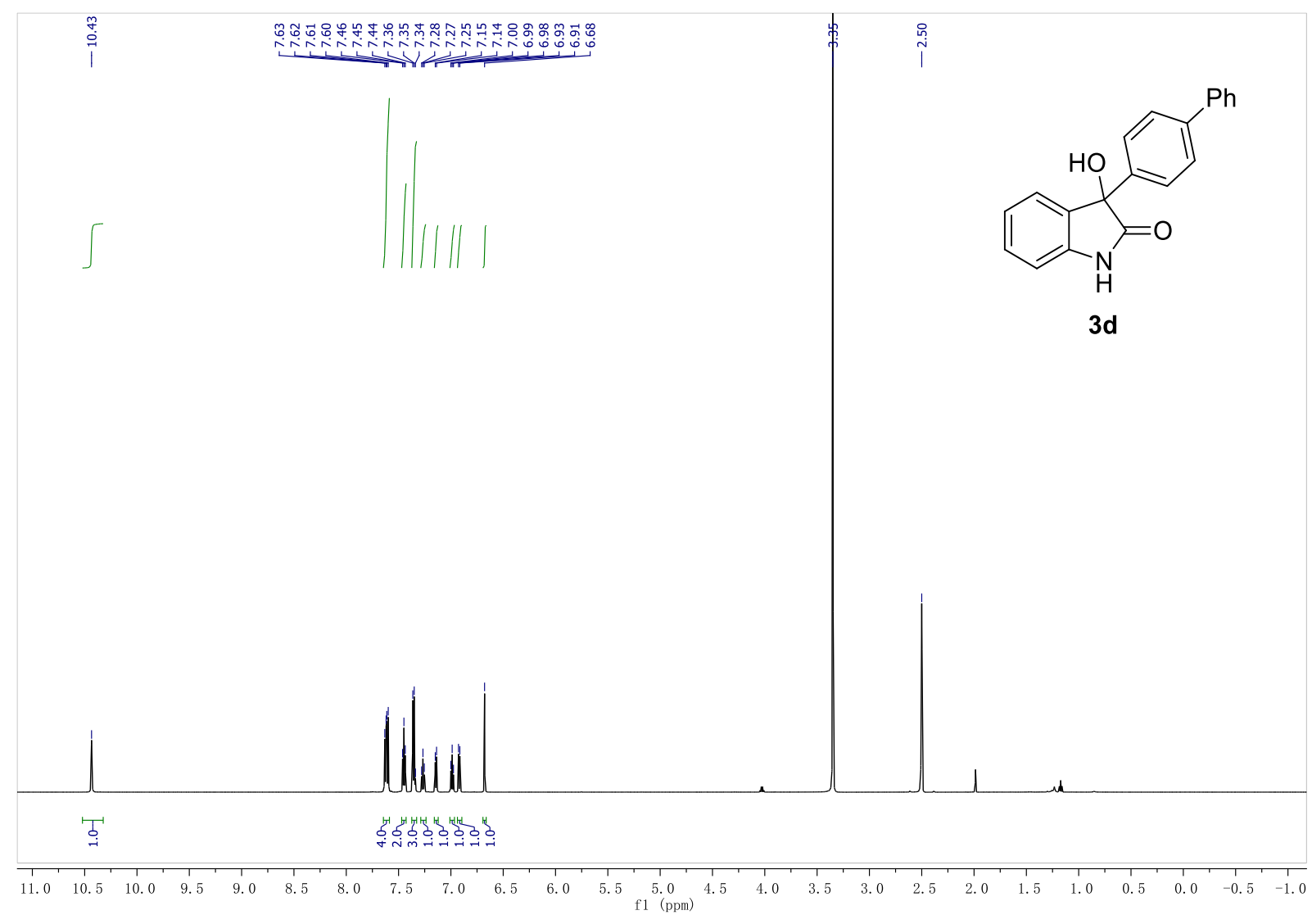

${ }^{1} \mathrm{H}$ NMR in DMSO- $d_{6}$ at $600 \mathrm{MHz}$

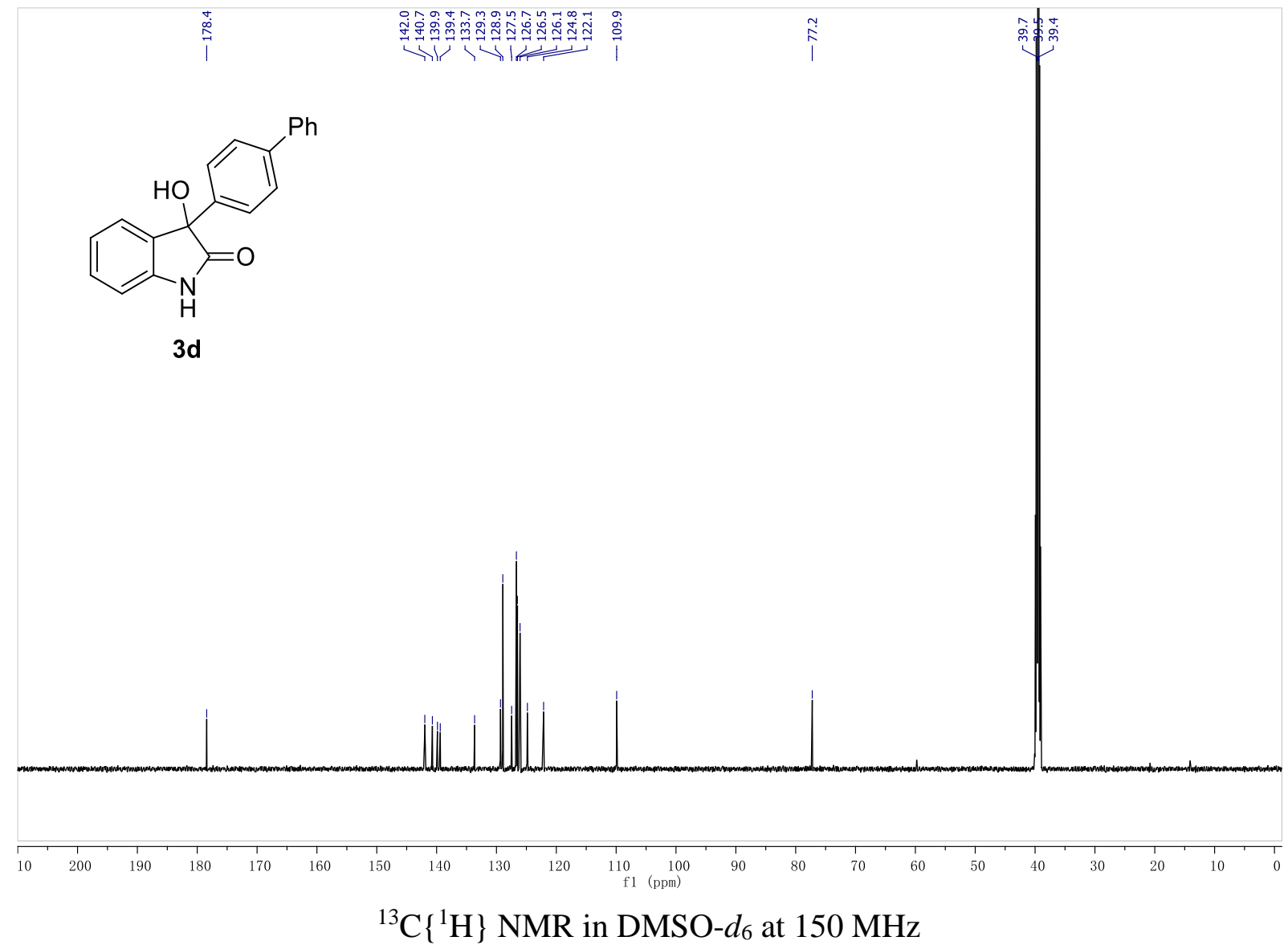




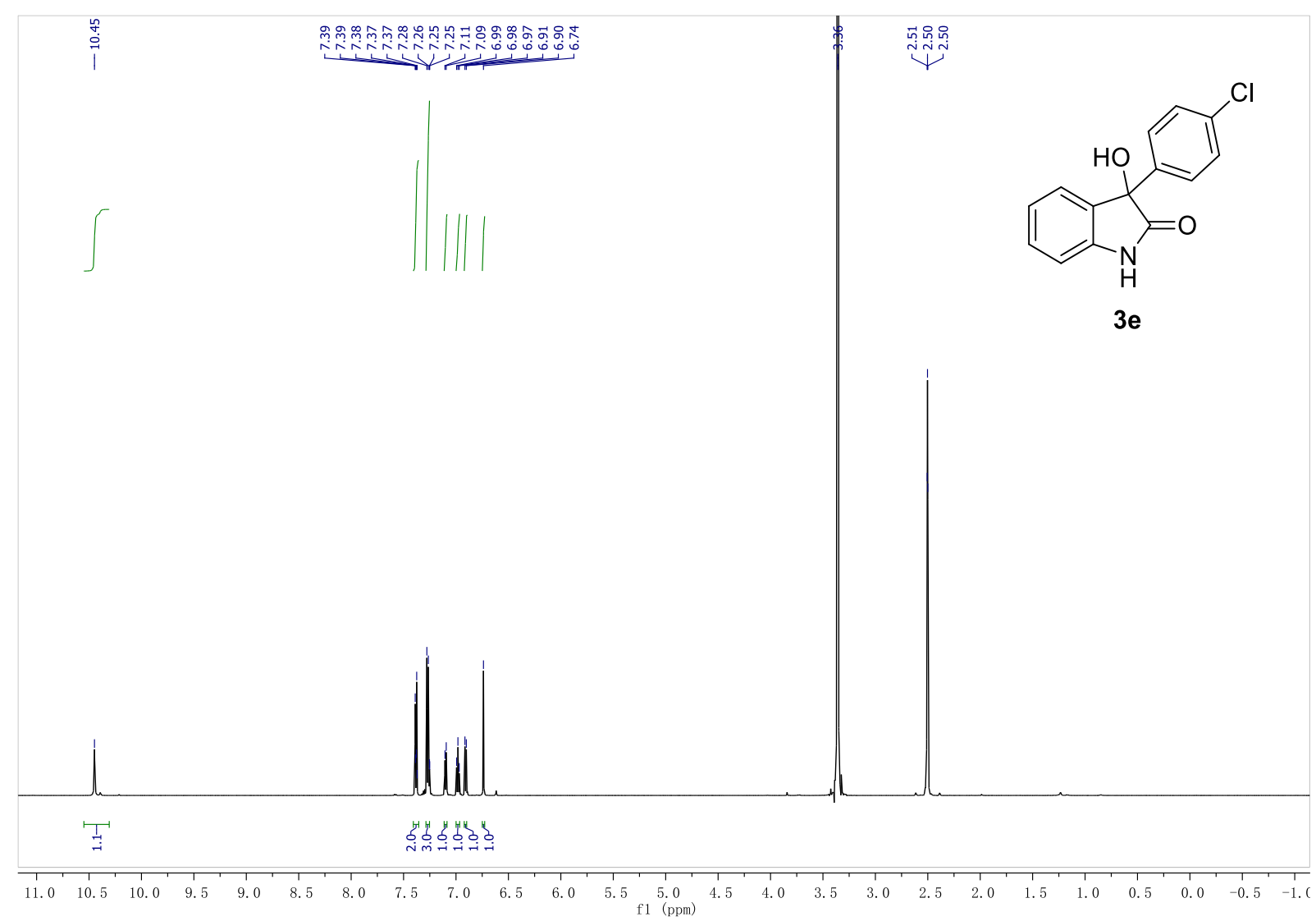

${ }^{1} \mathrm{H}$ NMR in DMSO- $d_{6}$ at $600 \mathrm{MHz}$
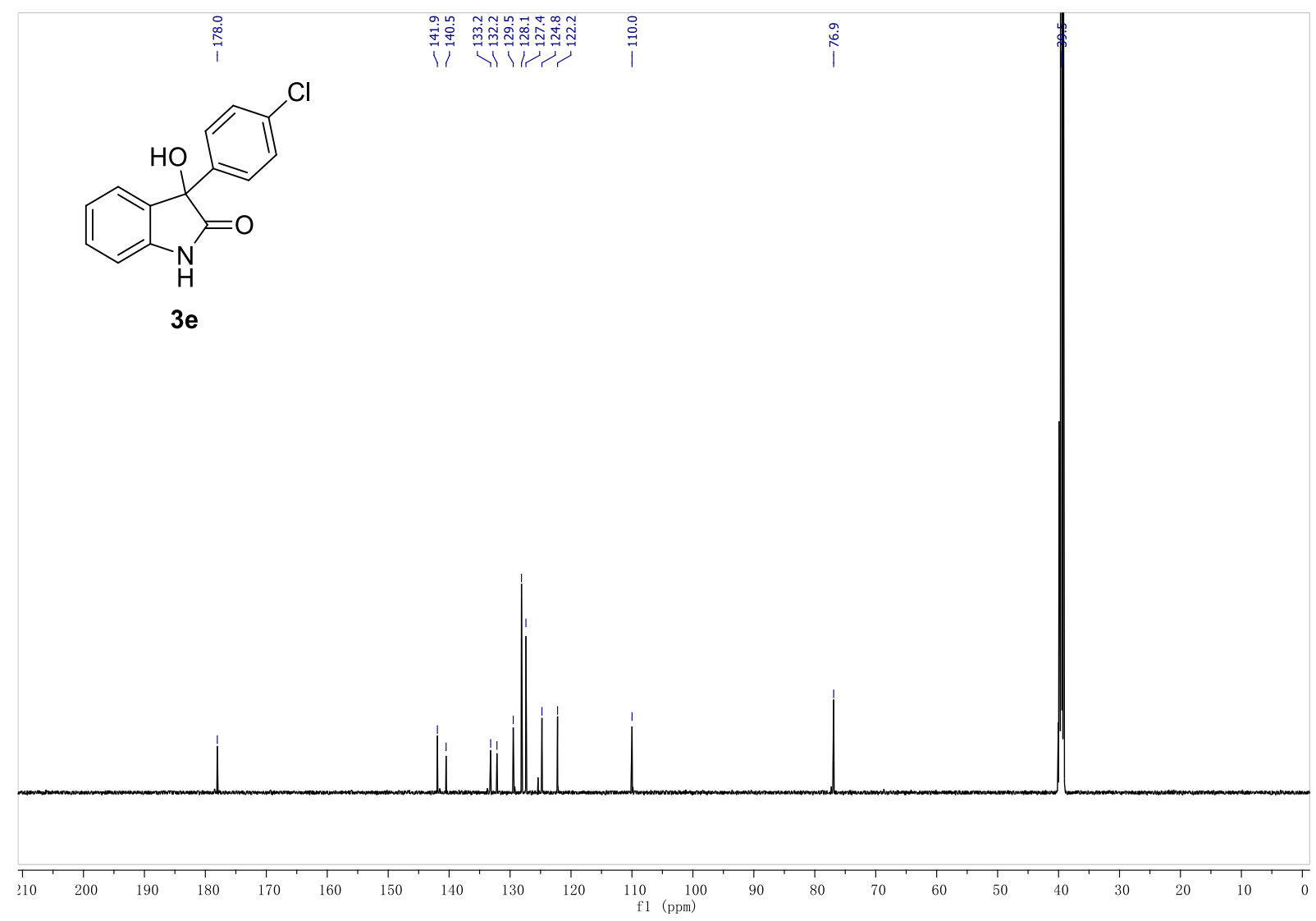

${ }^{13} \mathrm{C}\left\{{ }^{1} \mathrm{H}\right\}$ NMR in DMSO- $d_{6}$ at $150 \mathrm{MHz}$ 


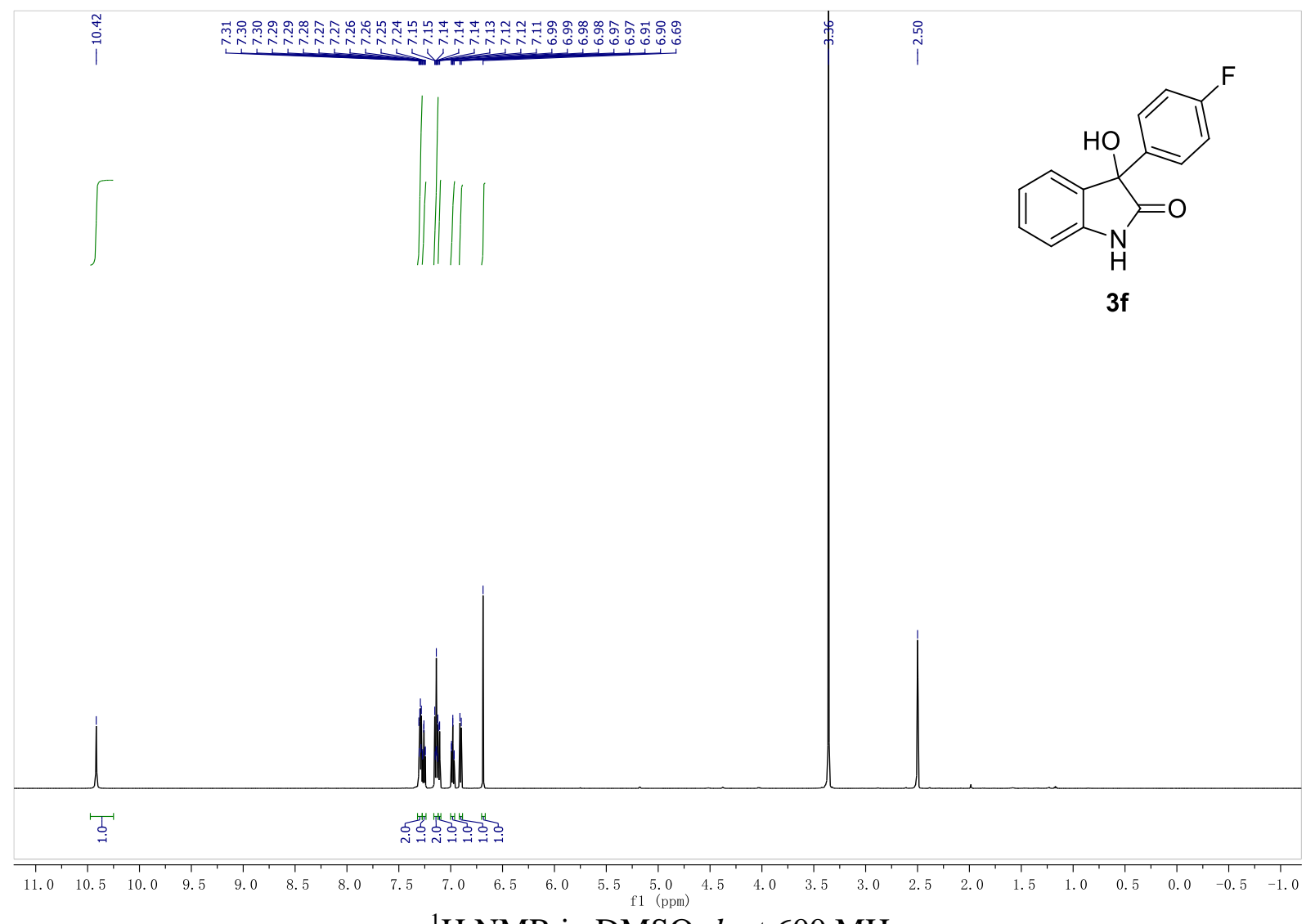

${ }^{1} \mathrm{H}$ NMR in DMSO- $d_{6}$ at $600 \mathrm{MHz}$

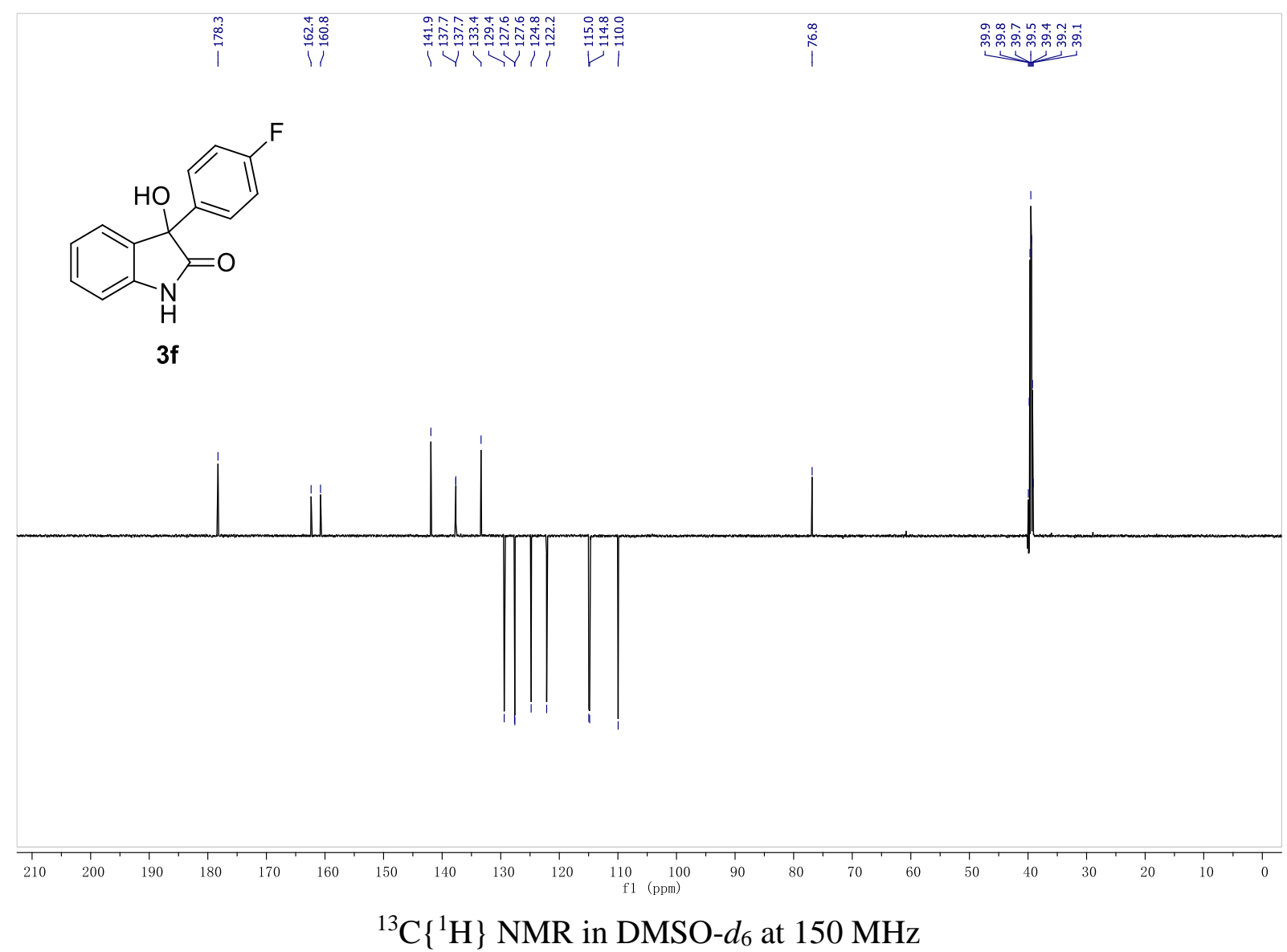




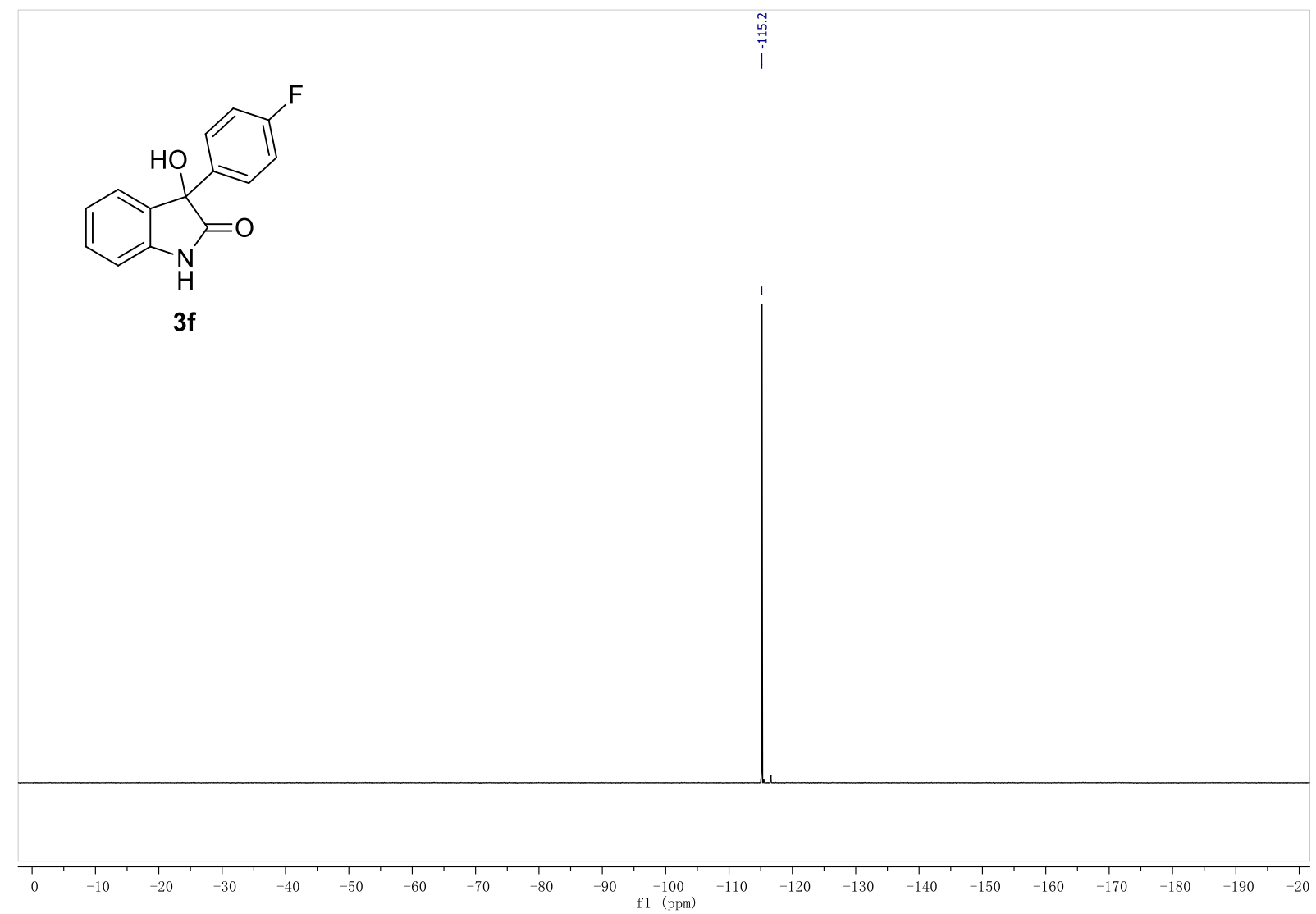

${ }^{19} \mathrm{~F}$ NMR in DMSO- $d_{6}$ at $565 \mathrm{MHz}$ 


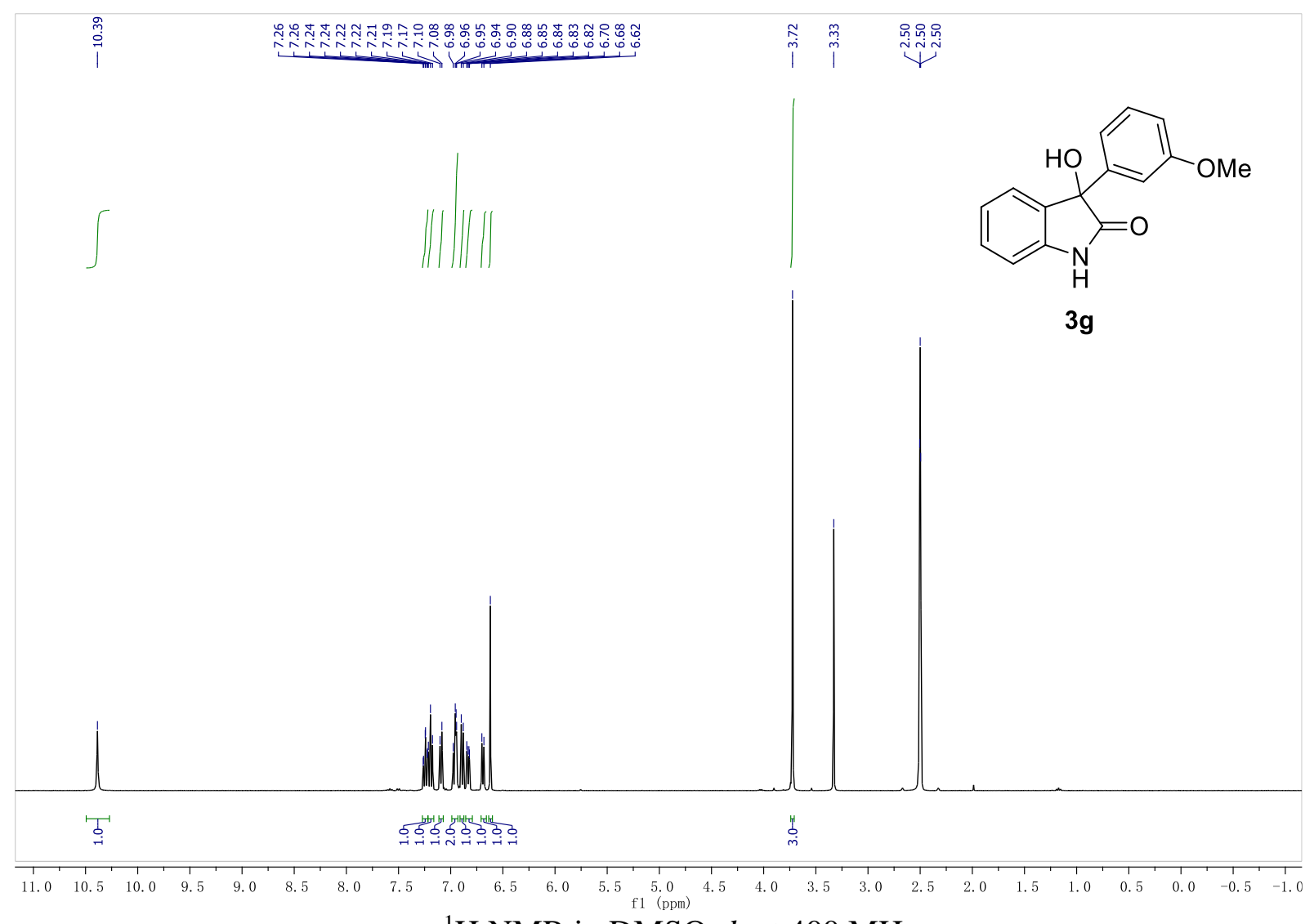

${ }^{1} \mathrm{H}$ NMR in DMSO- $d_{6}$ at $400 \mathrm{MHz}$

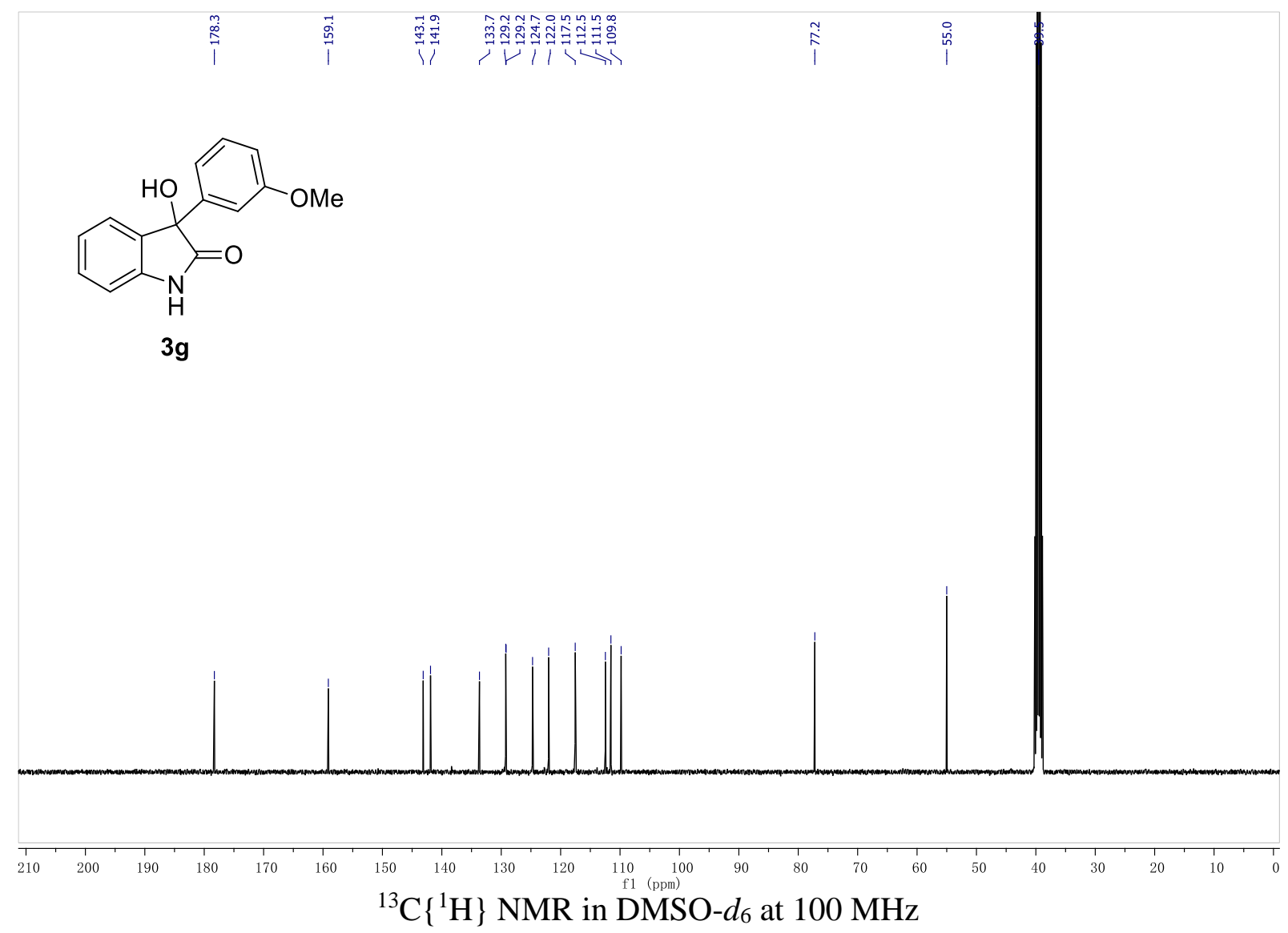




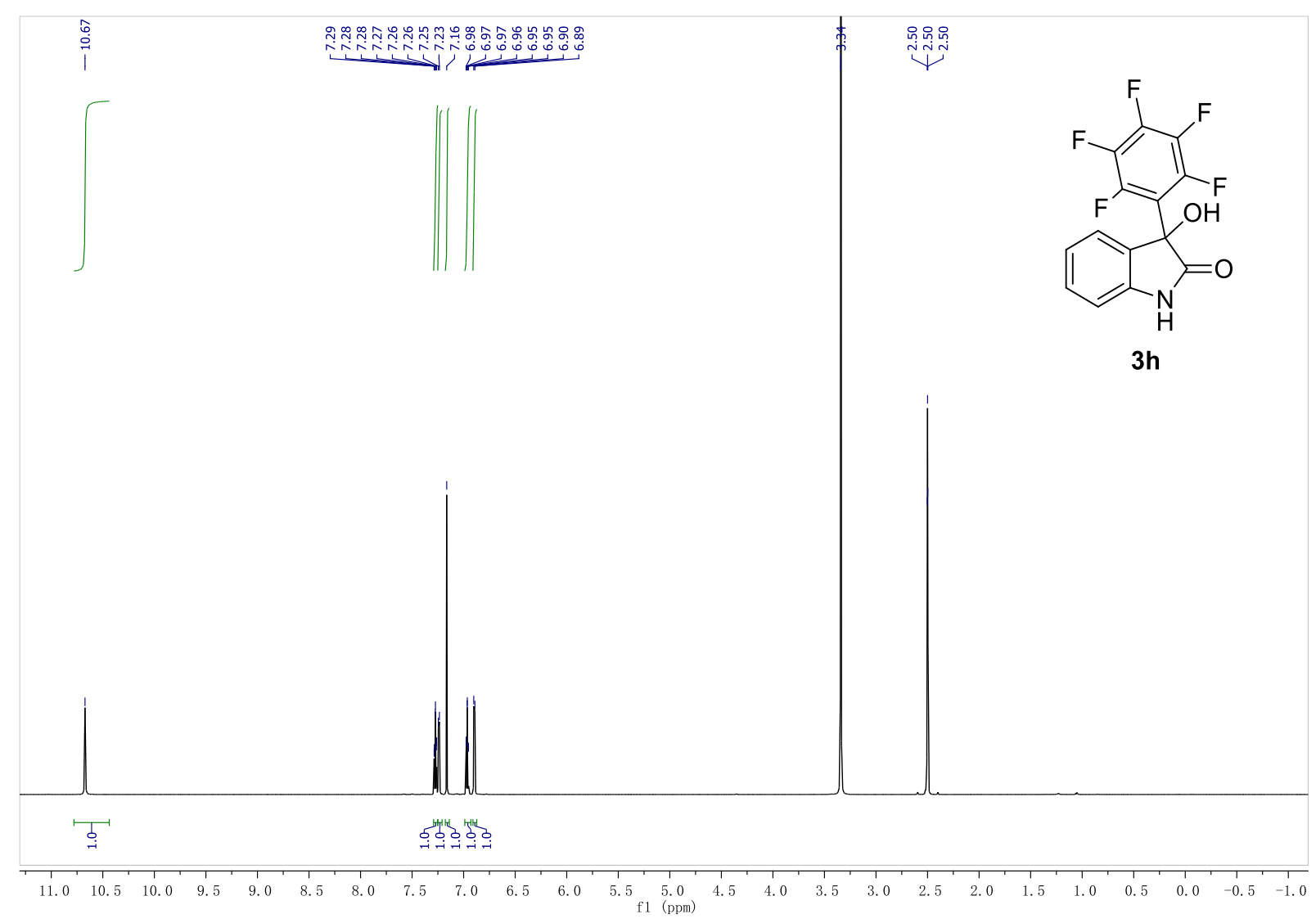

${ }^{1} \mathrm{H}$ NMR in DMSO- $d_{6}$ at $700 \mathrm{MHz}$

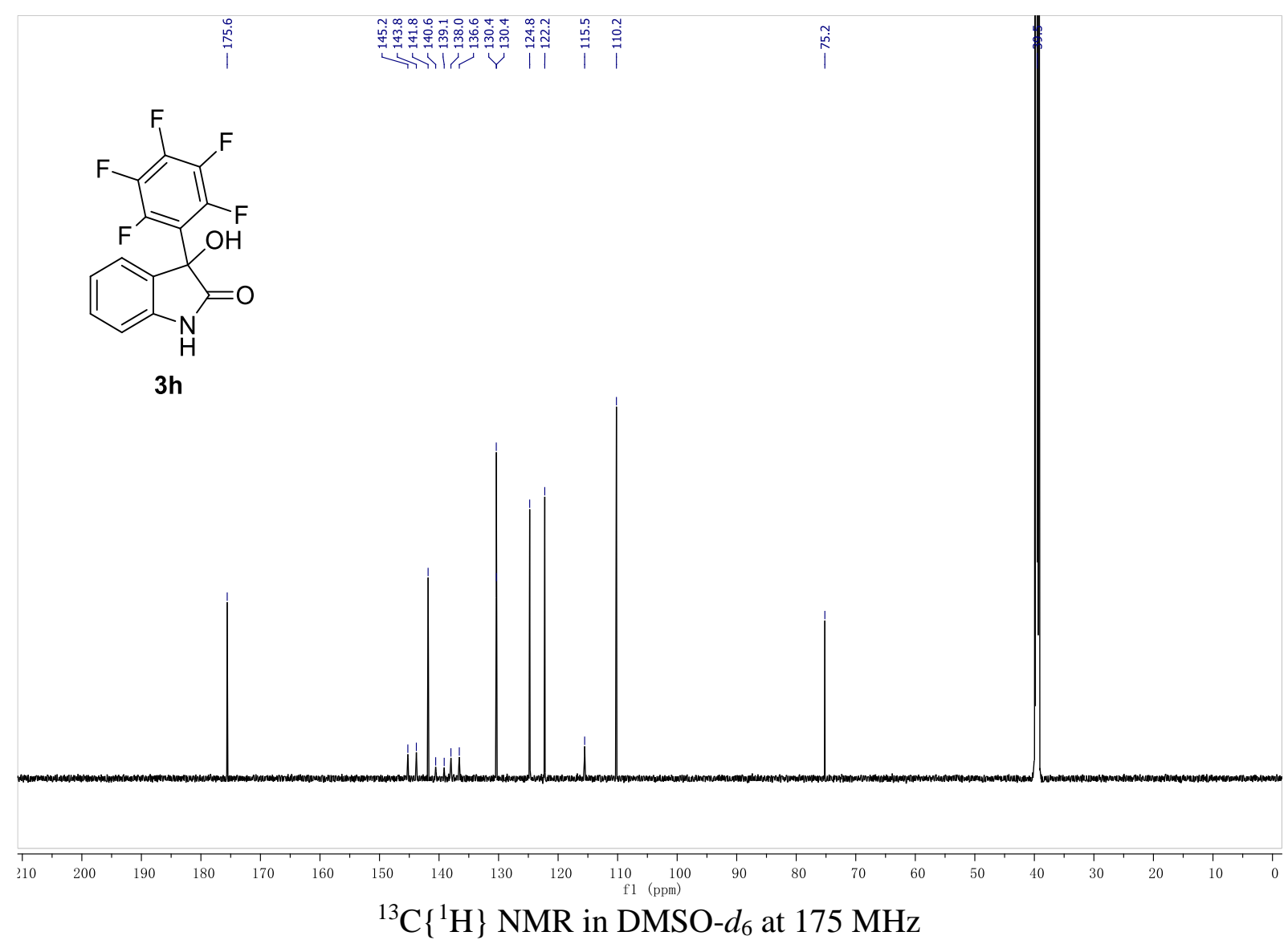




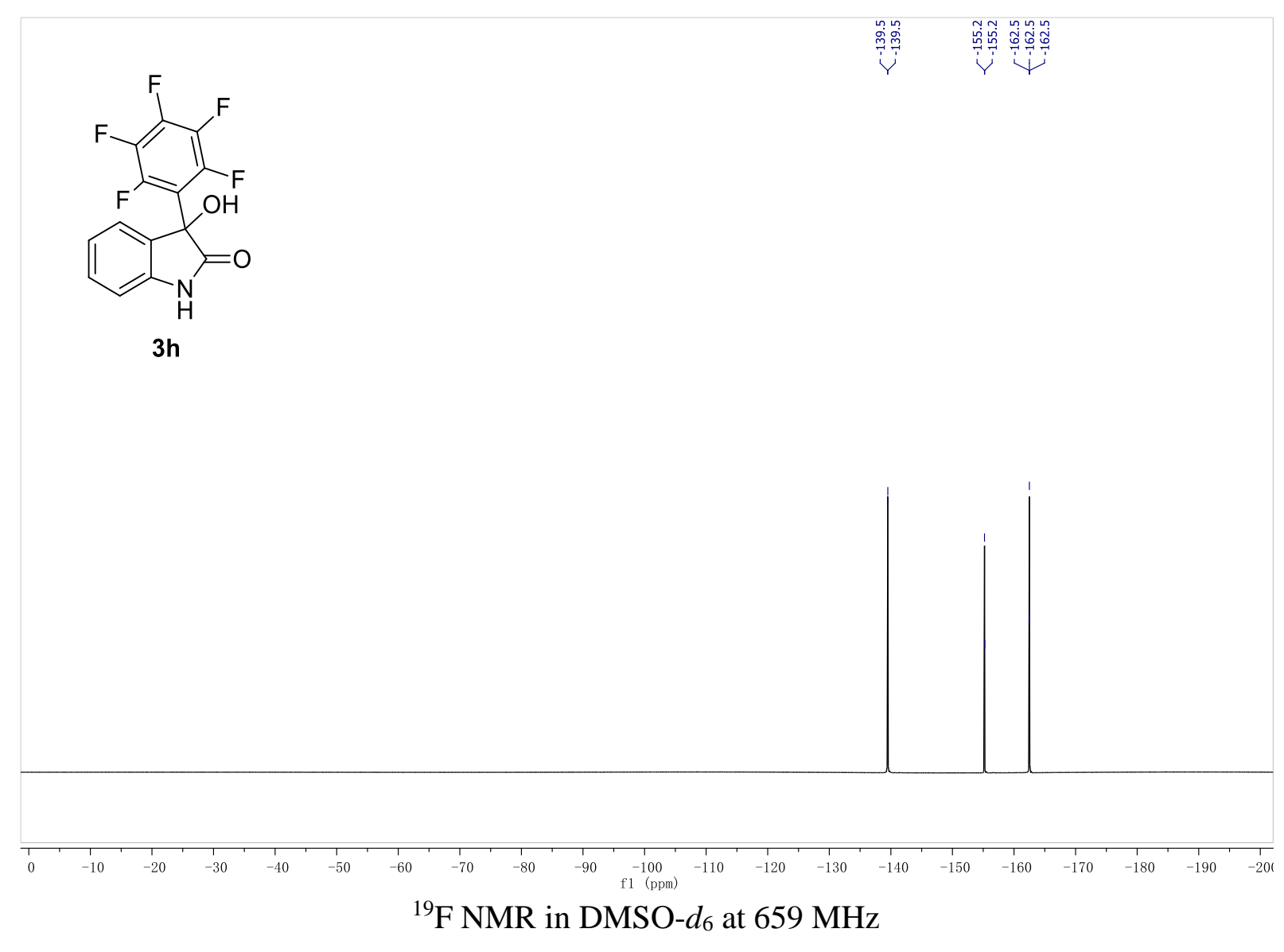




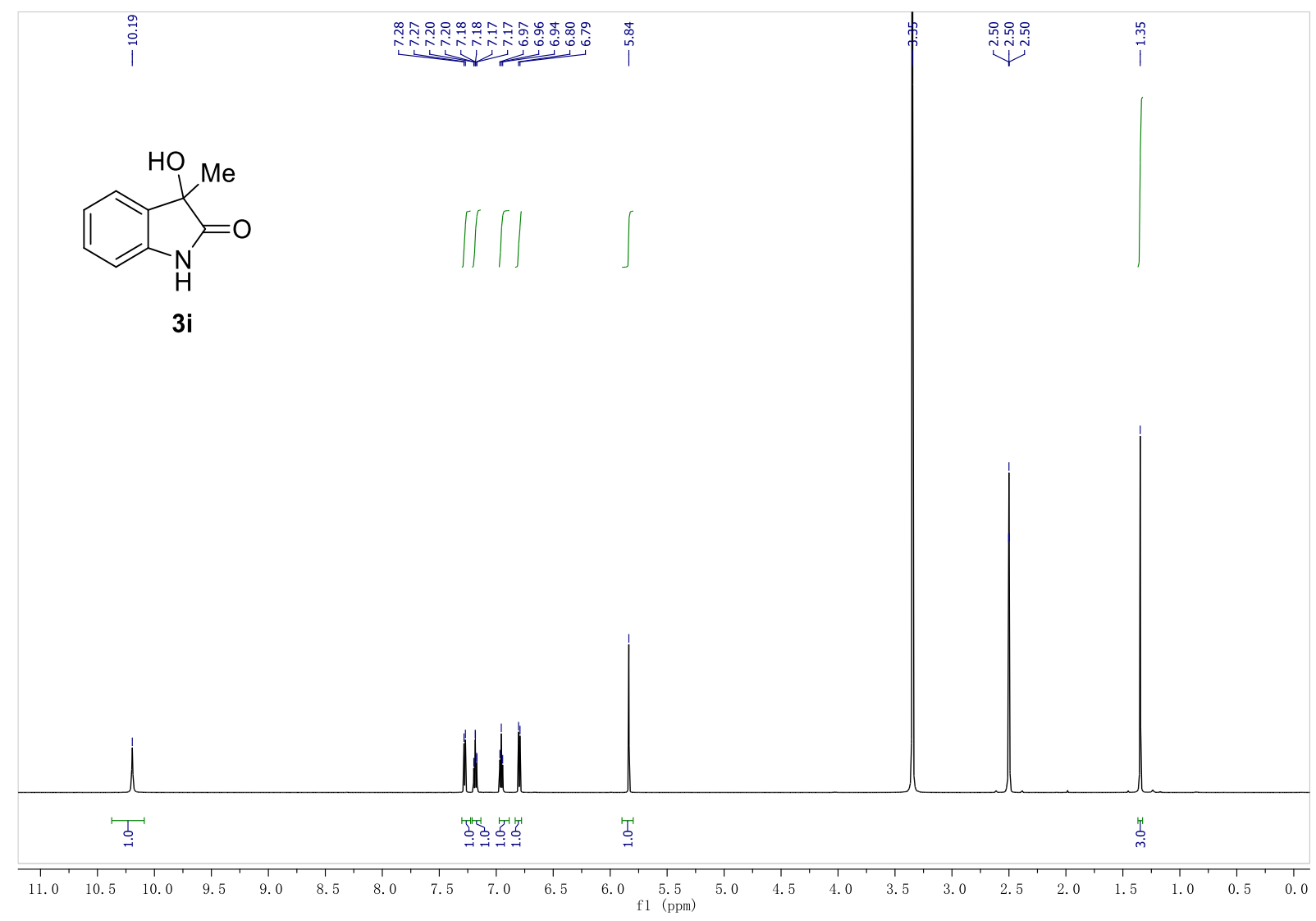

${ }^{1} \mathrm{H}$ NMR in DMSO- $d_{6}$ at $600 \mathrm{MHz}$

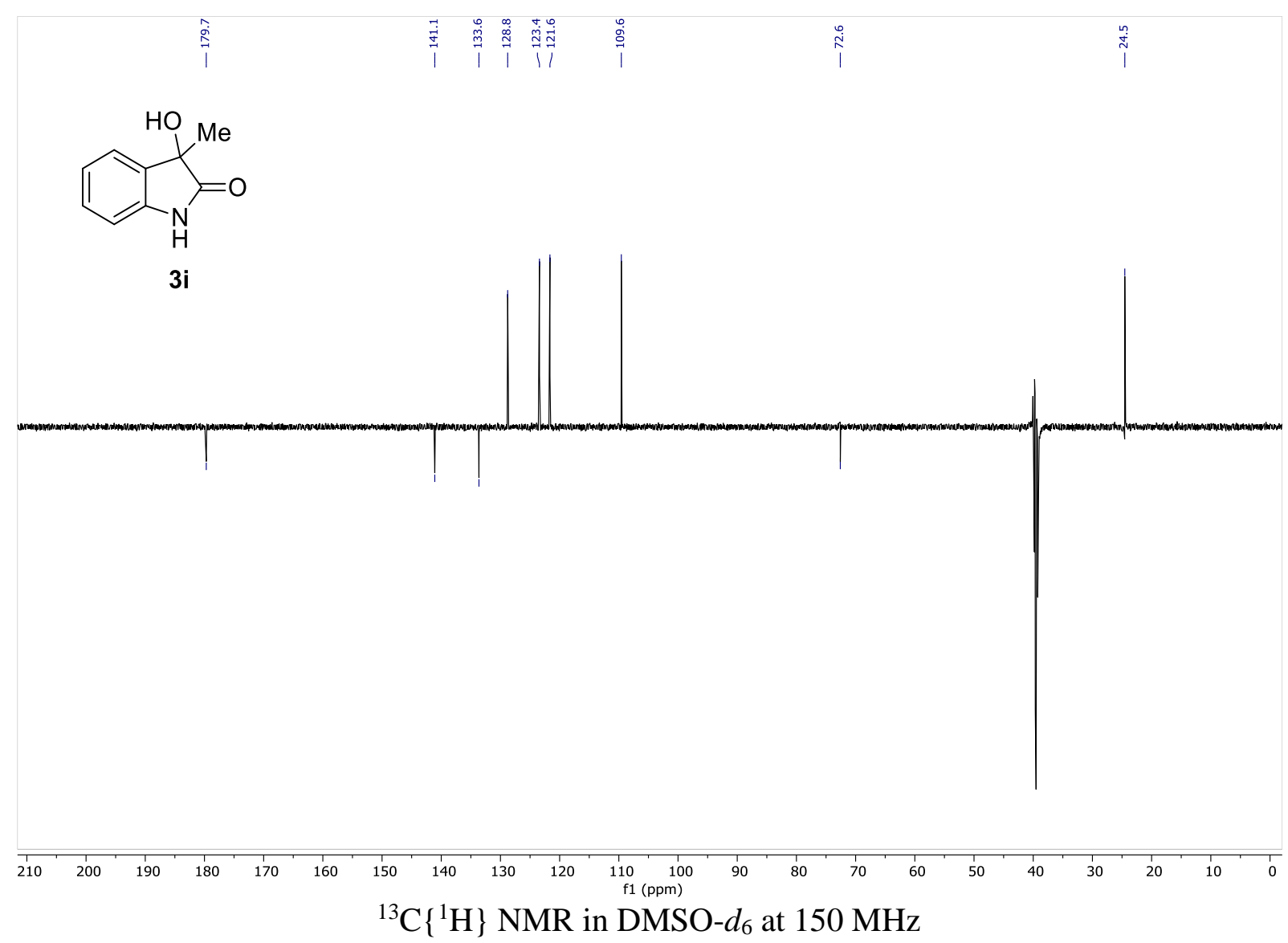




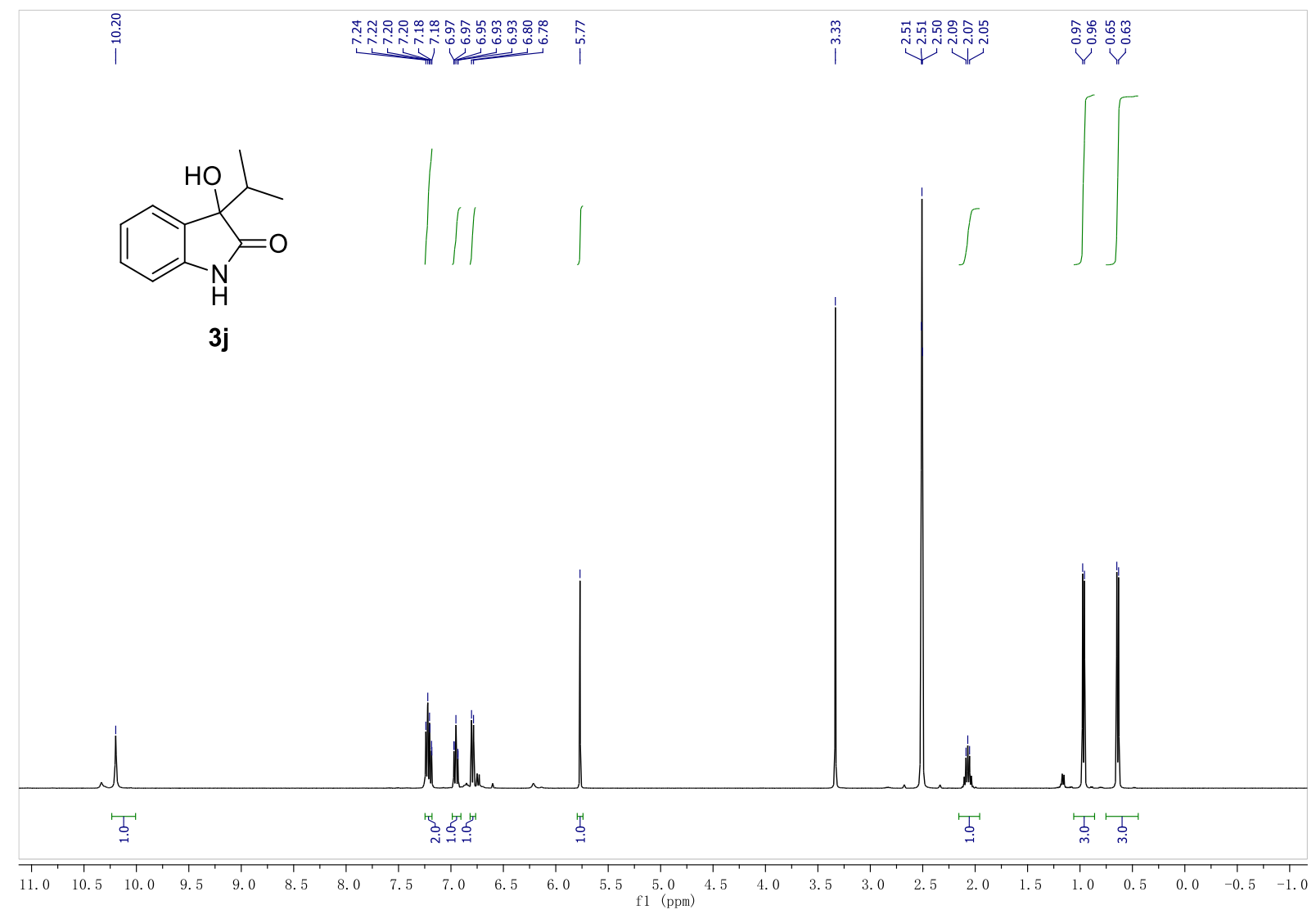

${ }^{1} \mathrm{H}$ NMR in DMSO- $d_{6}$ at $400 \mathrm{MHz}$

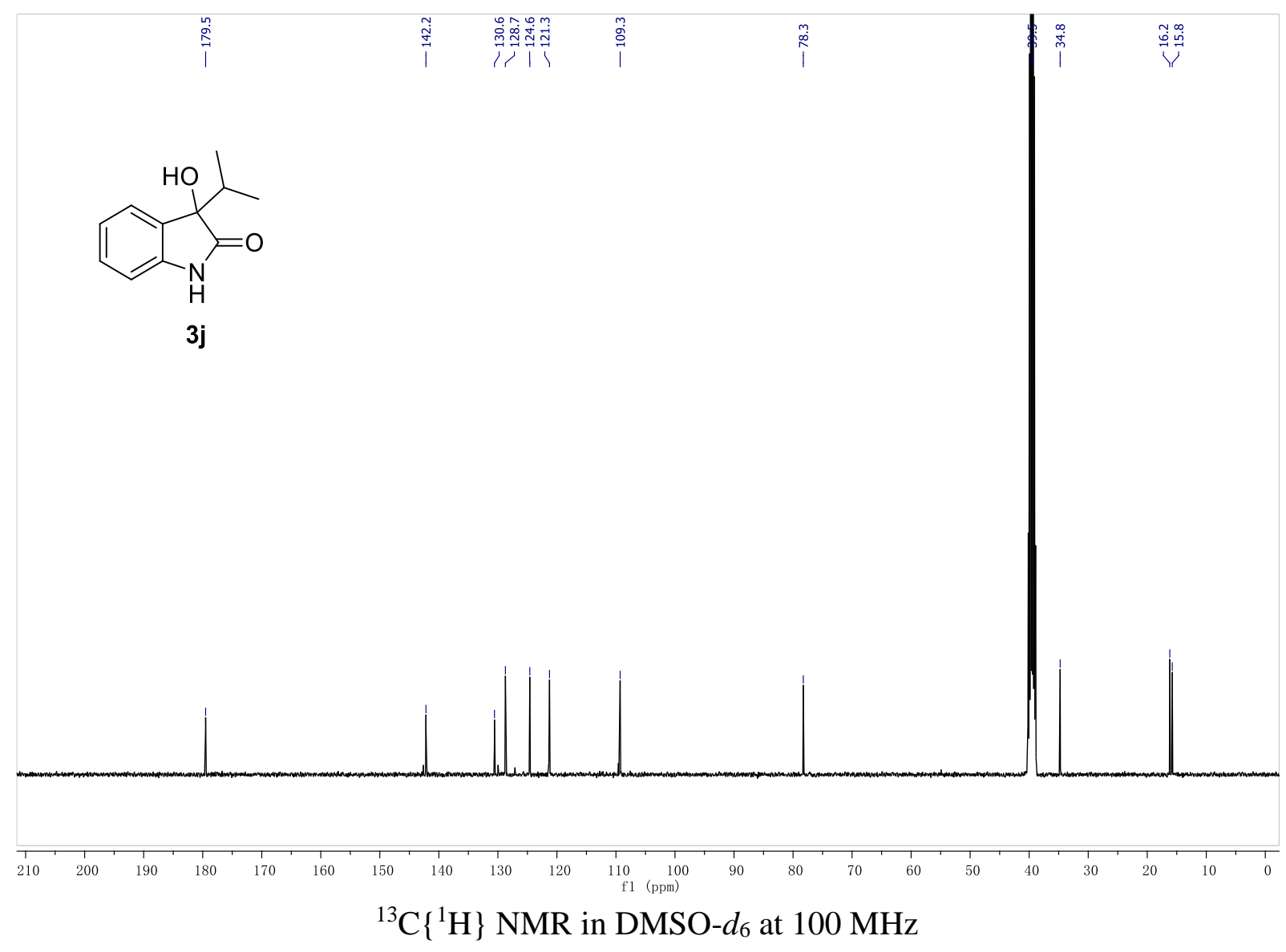




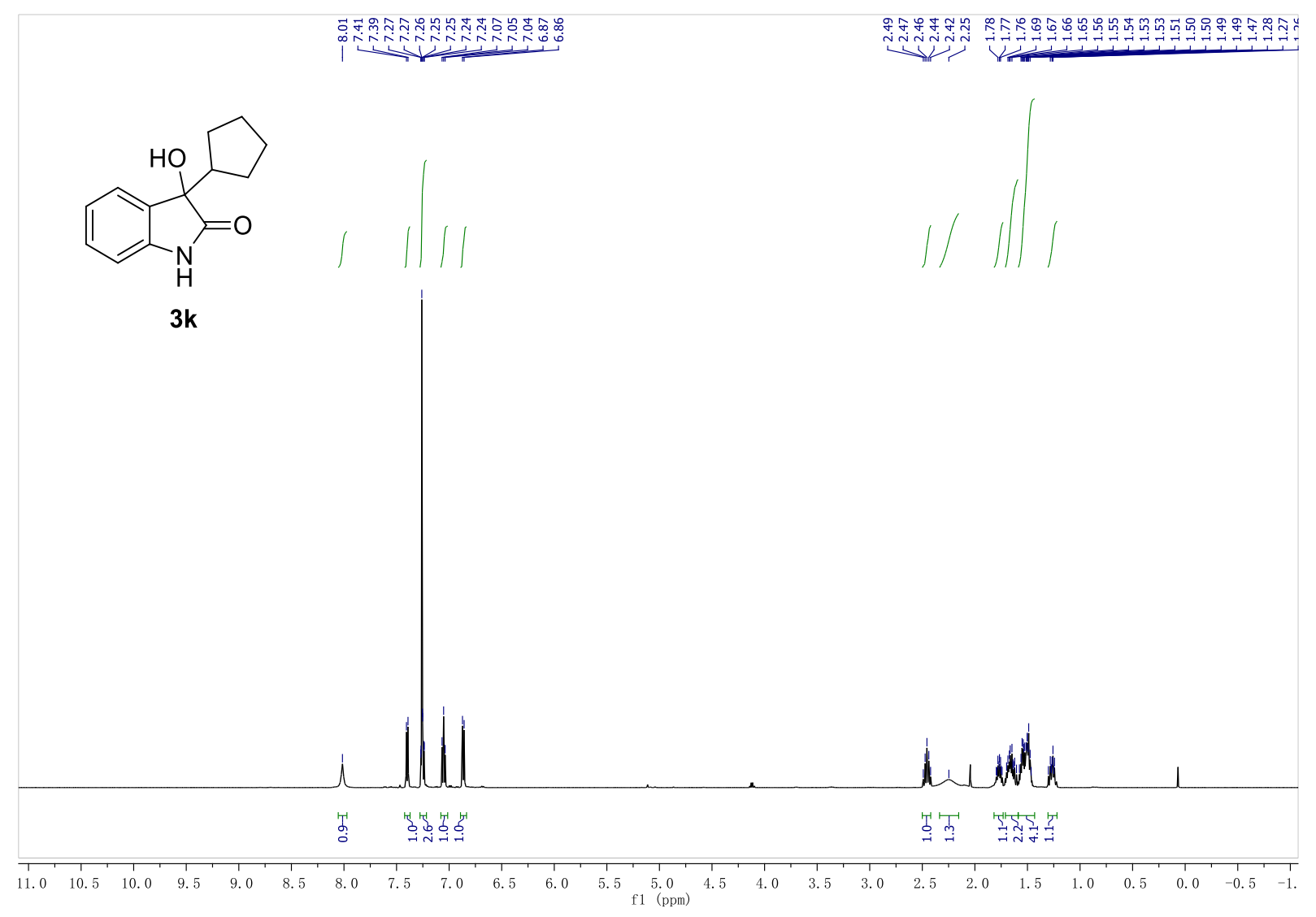

${ }^{1} \mathrm{H} \mathrm{NMR}$ in $\mathrm{CDCl}_{3}$ at $400 \mathrm{MHz}$

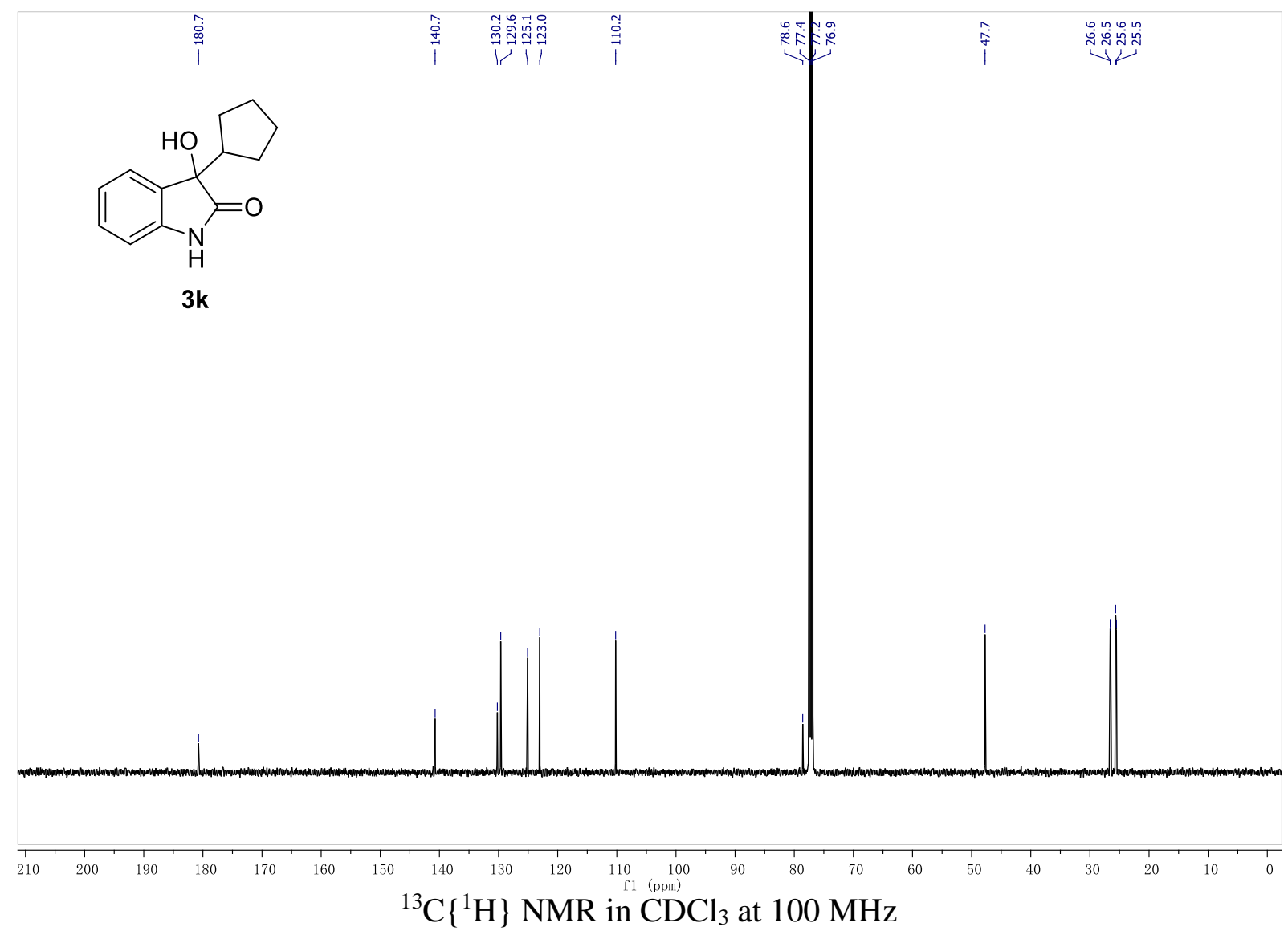




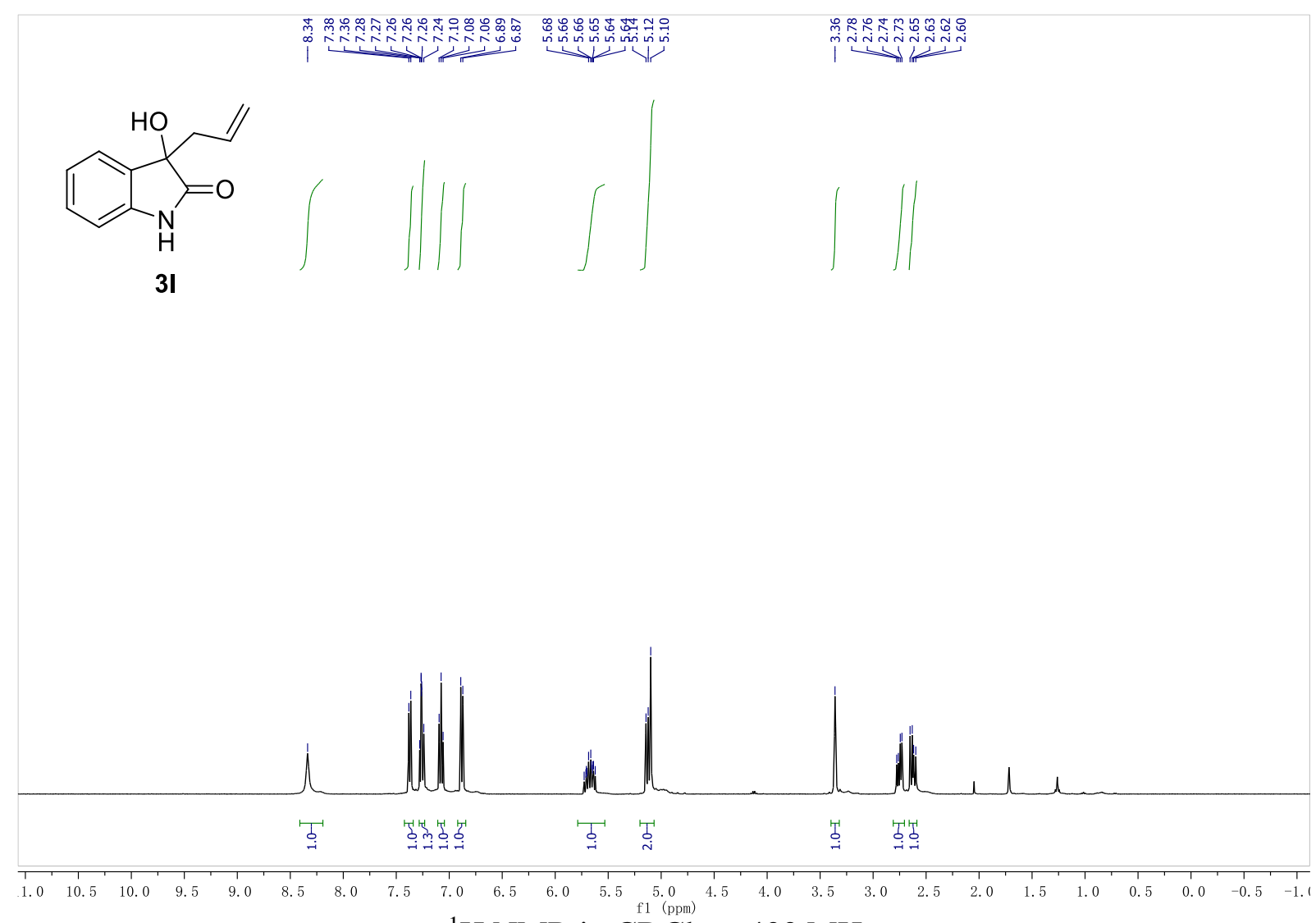

${ }^{1} \mathrm{H} \mathrm{NMR}$ in $\mathrm{CDCl}_{3}$ at $400 \mathrm{MHz}$

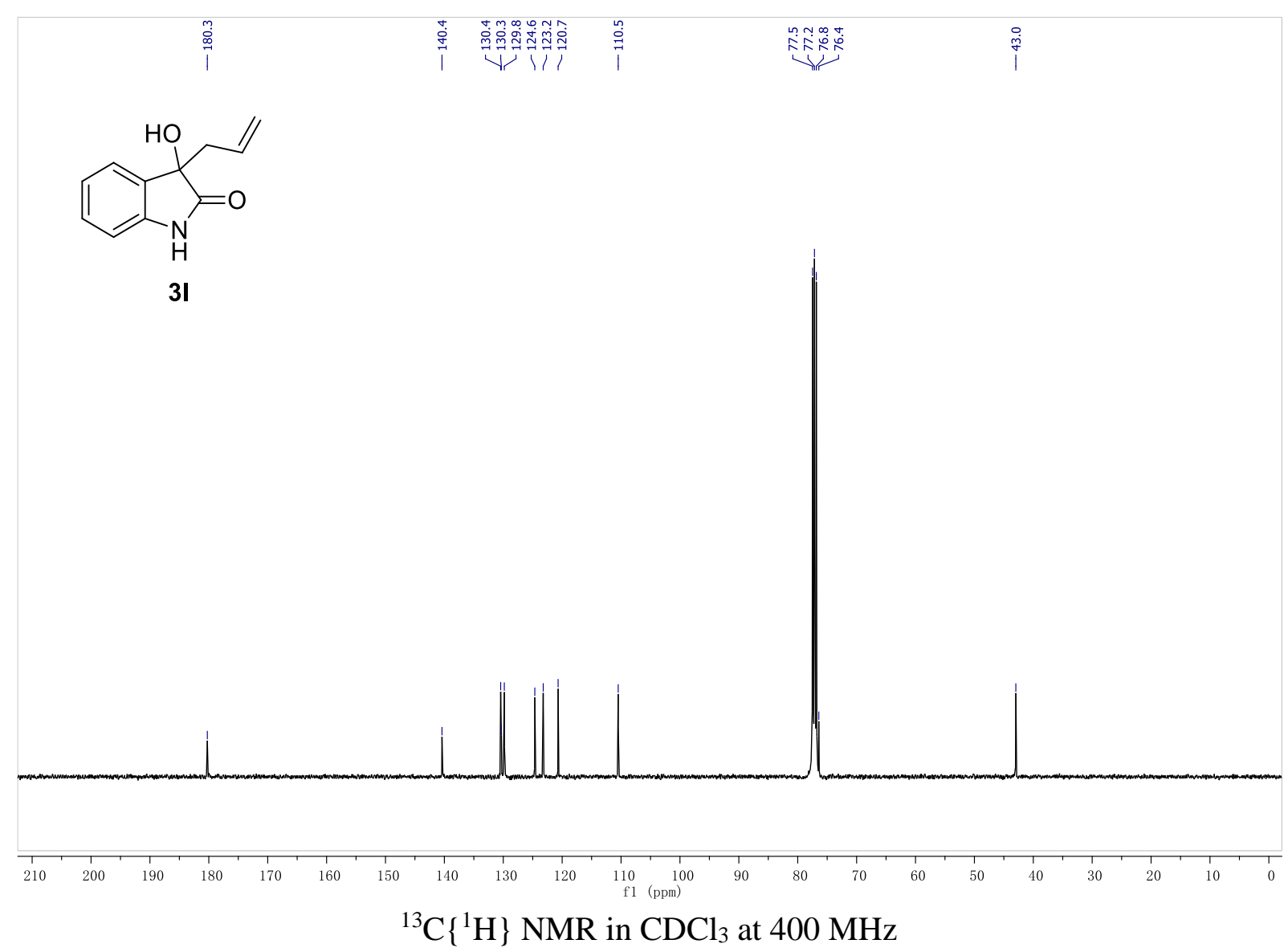




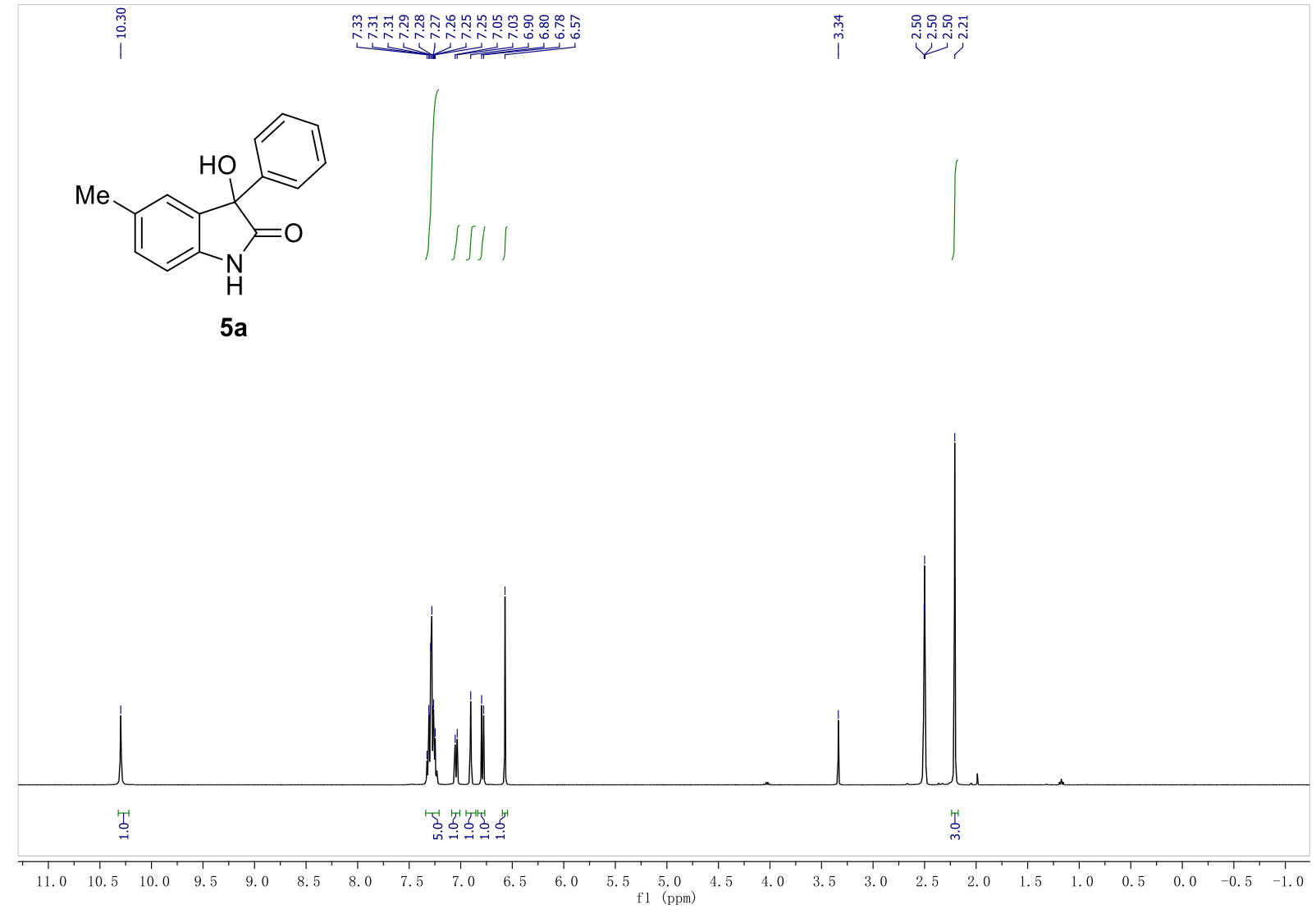

${ }^{1} \mathrm{H}$ NMR in DMSO- $d_{6}$ at $400 \mathrm{MHz}$

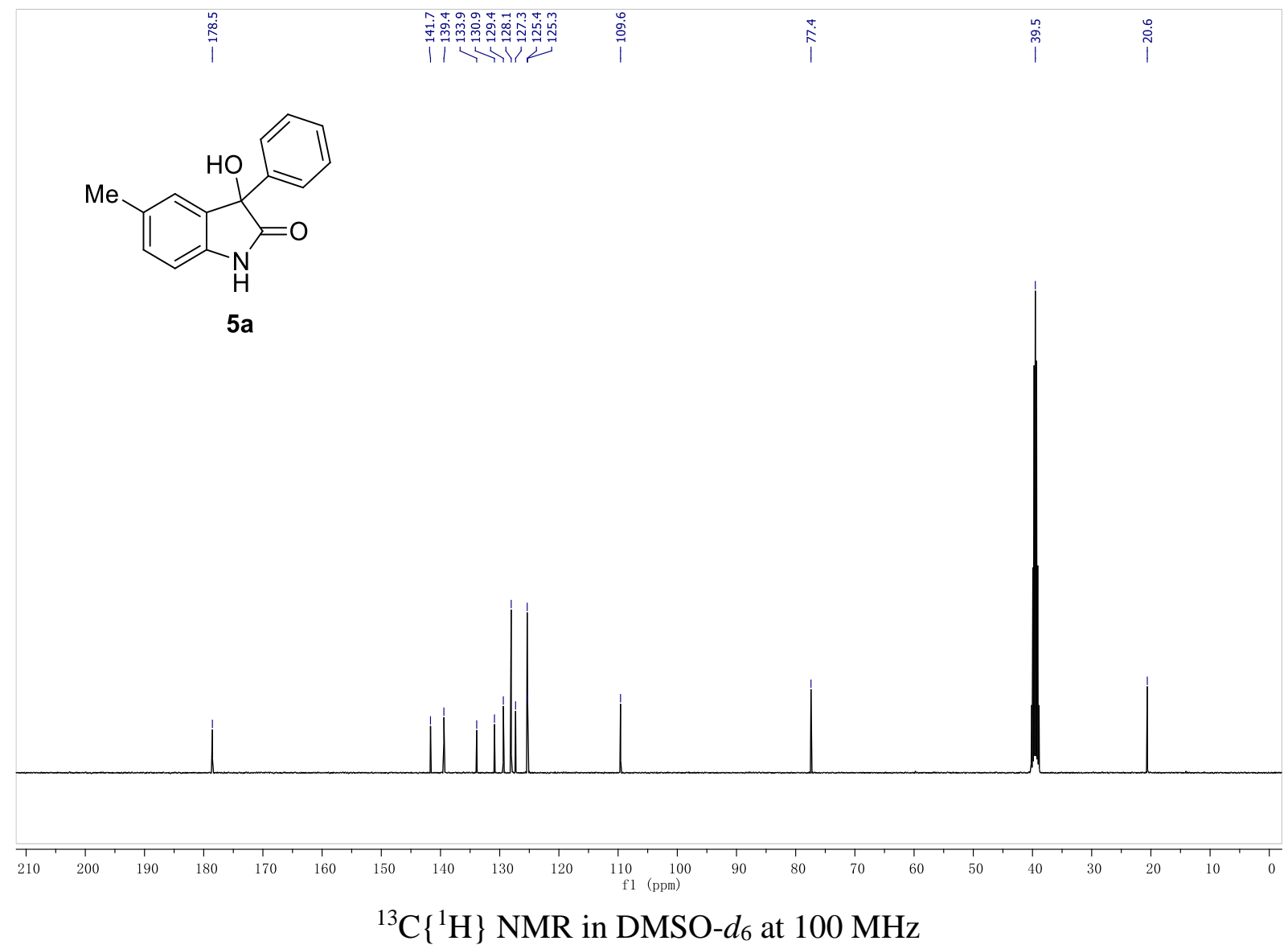




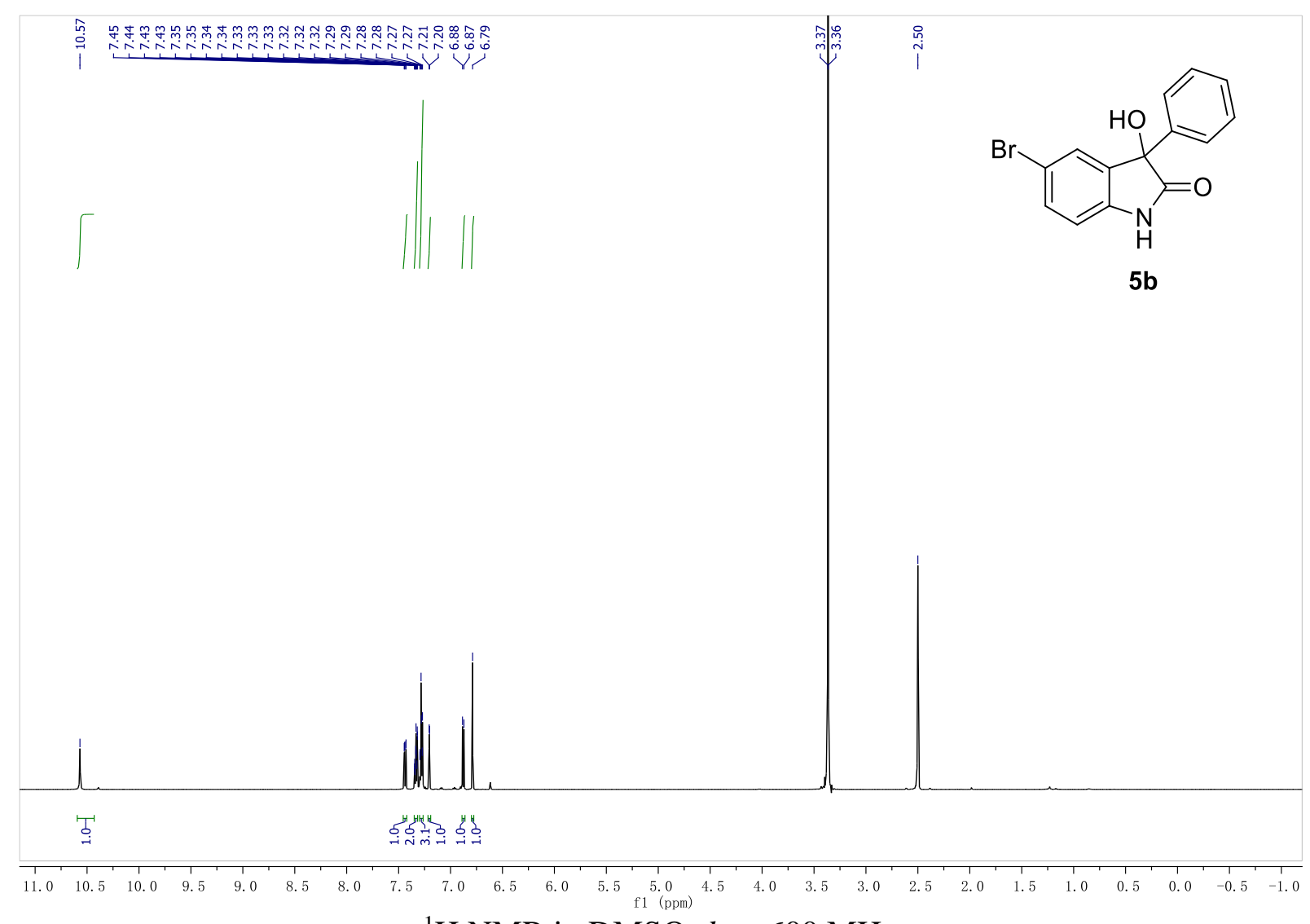

${ }^{1} \mathrm{H}$ NMR in DMSO- $d_{6}$ at $600 \mathrm{MHz}$

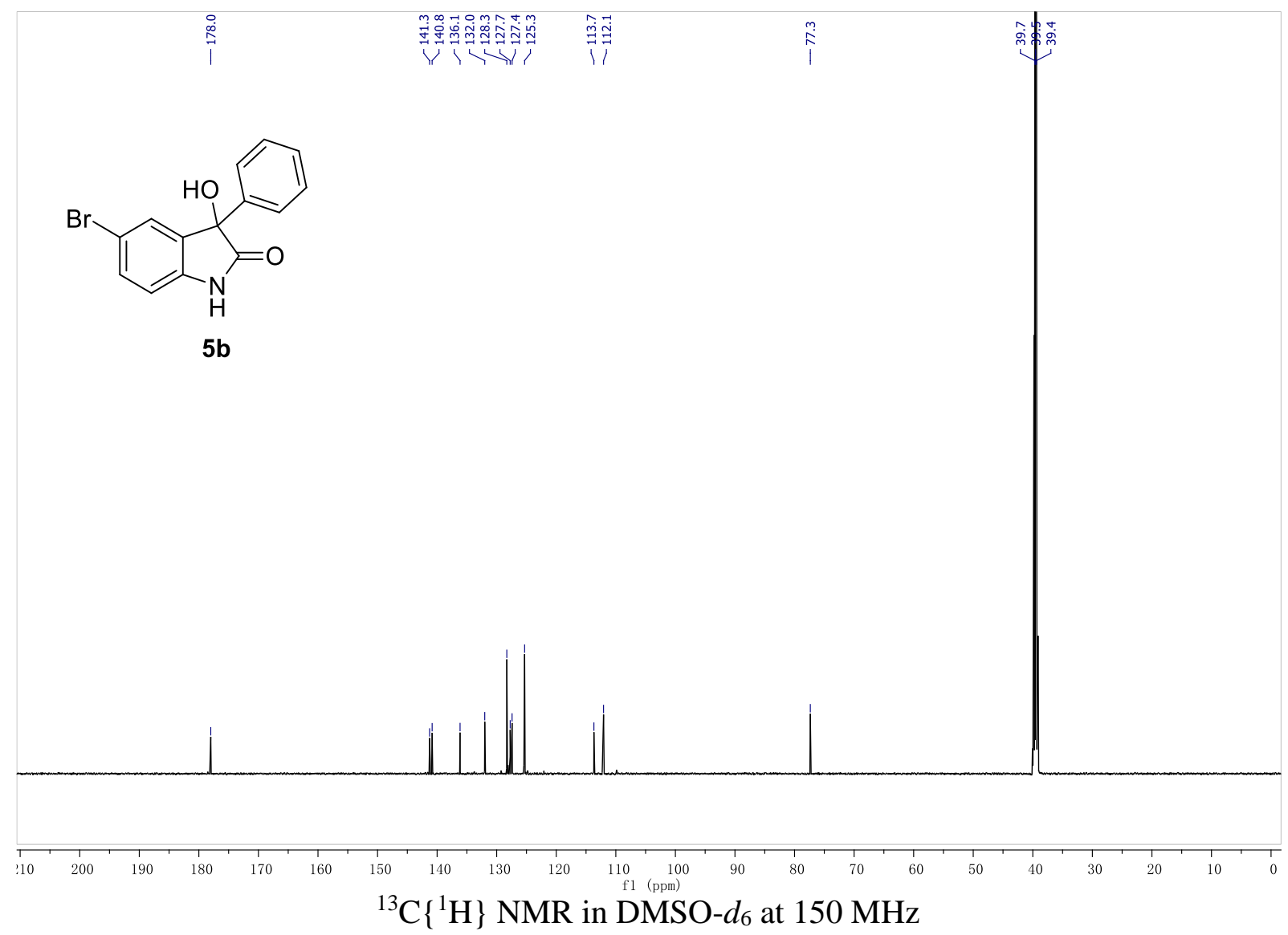




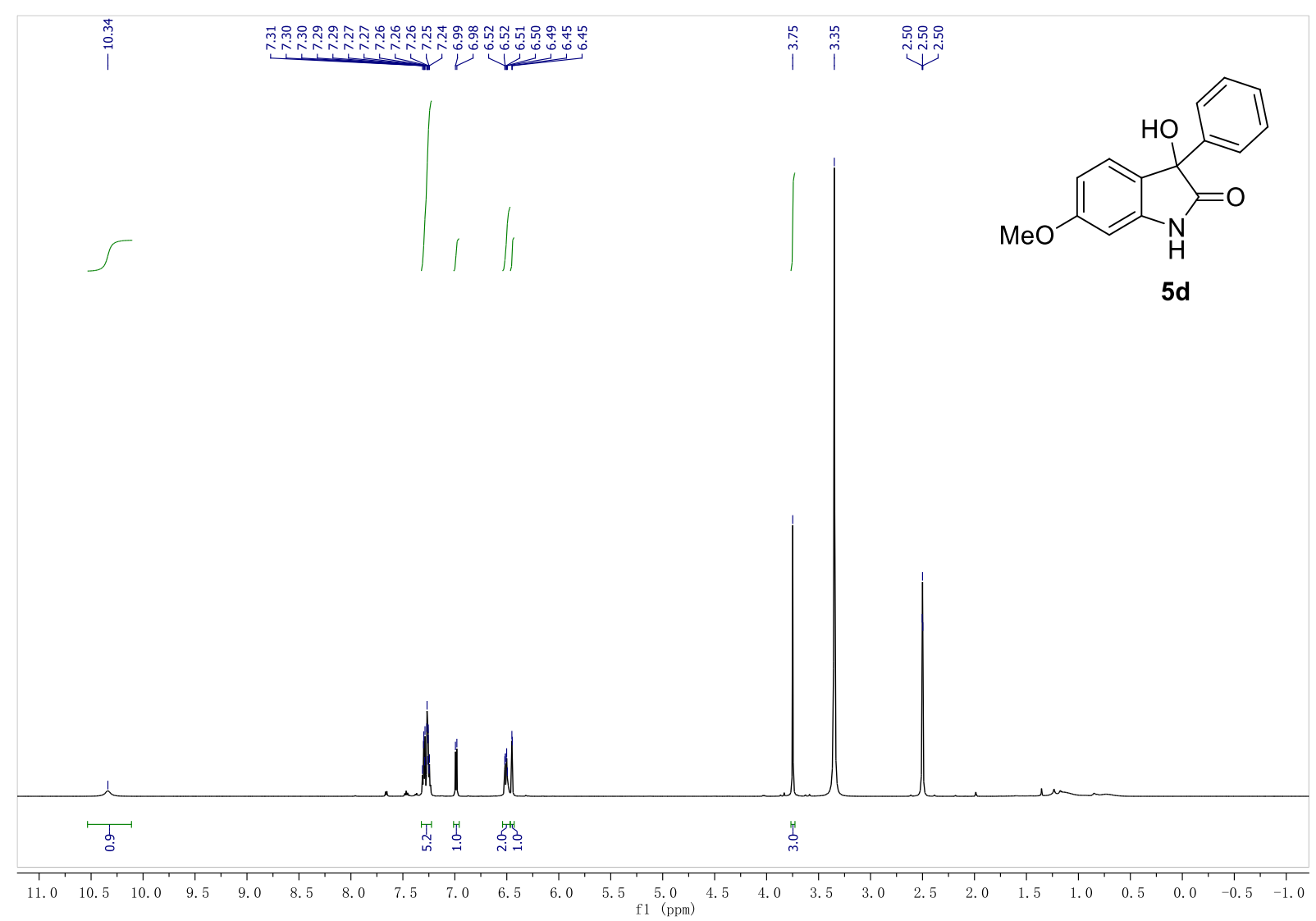

${ }^{1} \mathrm{H}$ NMR in DMSO- $d_{6}$ at $600 \mathrm{MHz}$

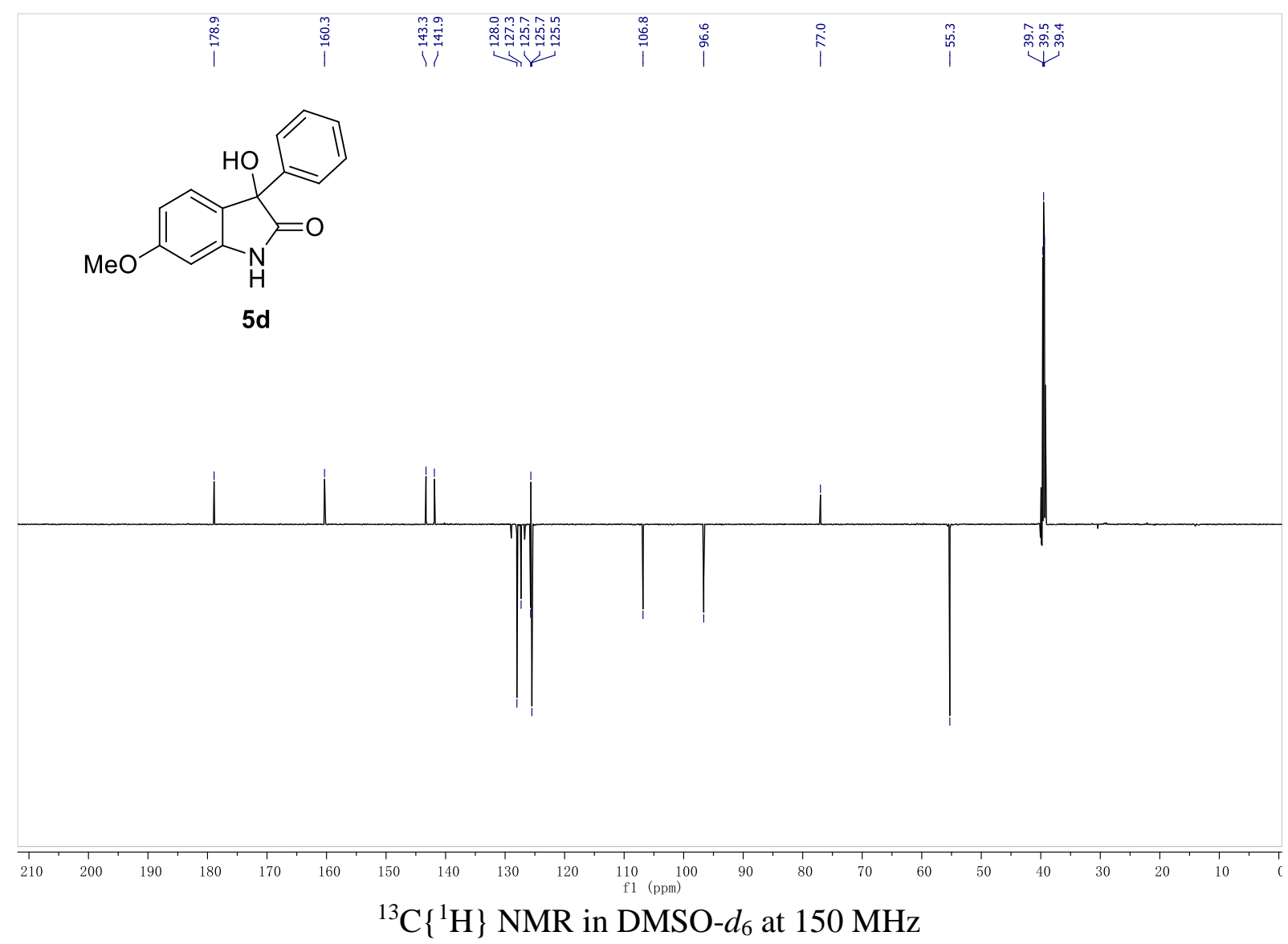




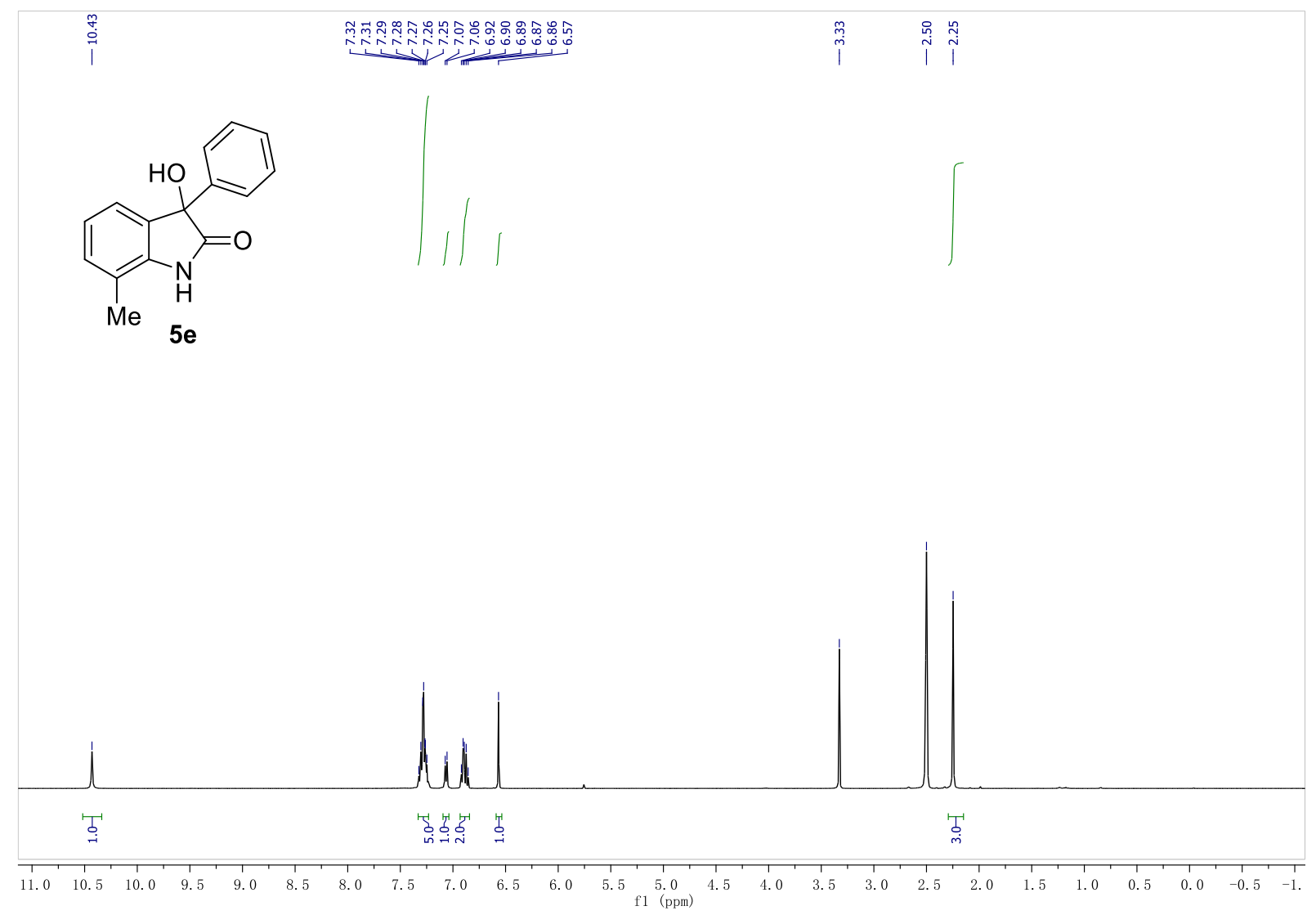

${ }^{1} \mathrm{H}$ NMR in DMSO- $d_{6}$ at $400 \mathrm{MHz}$

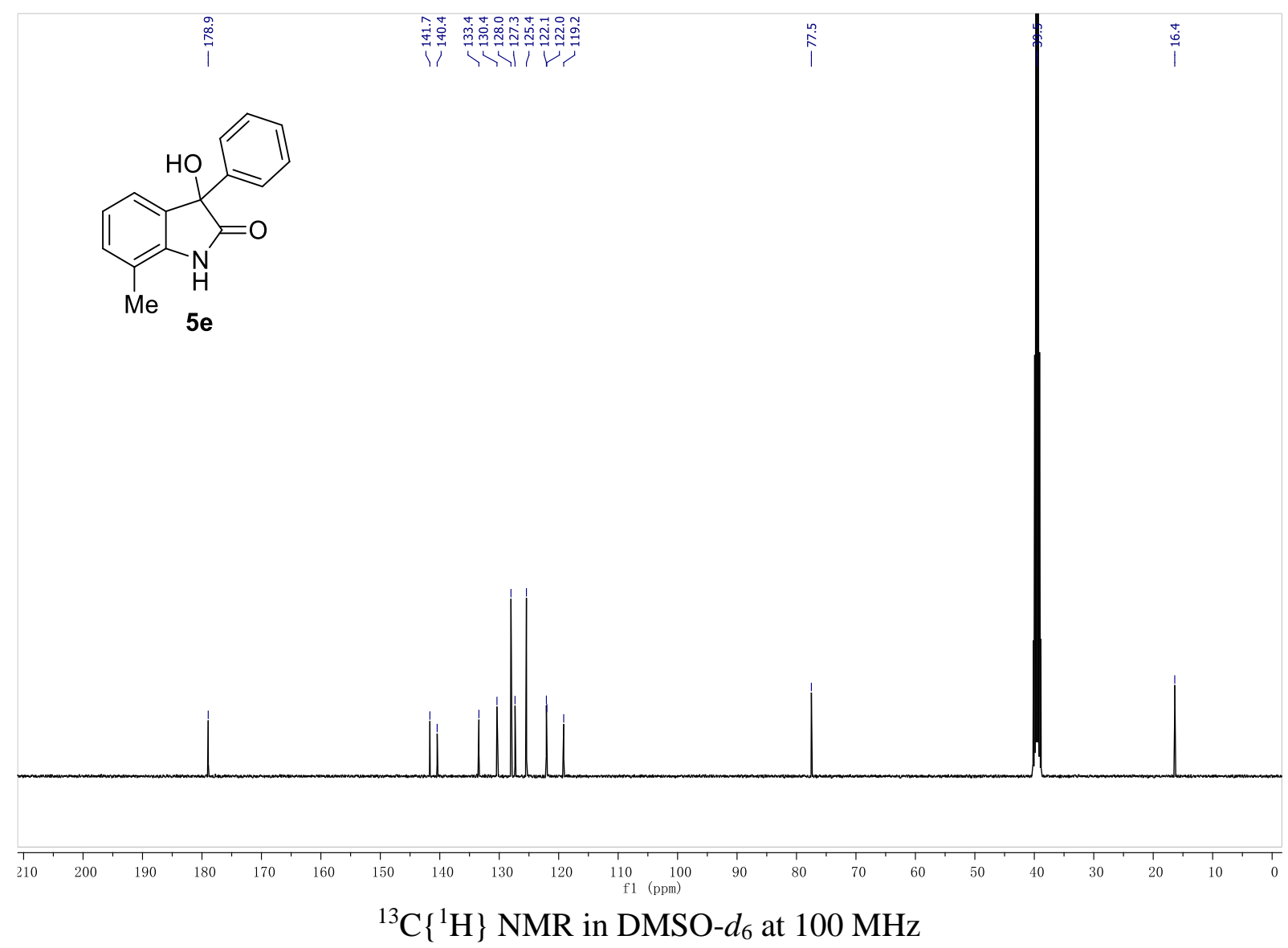




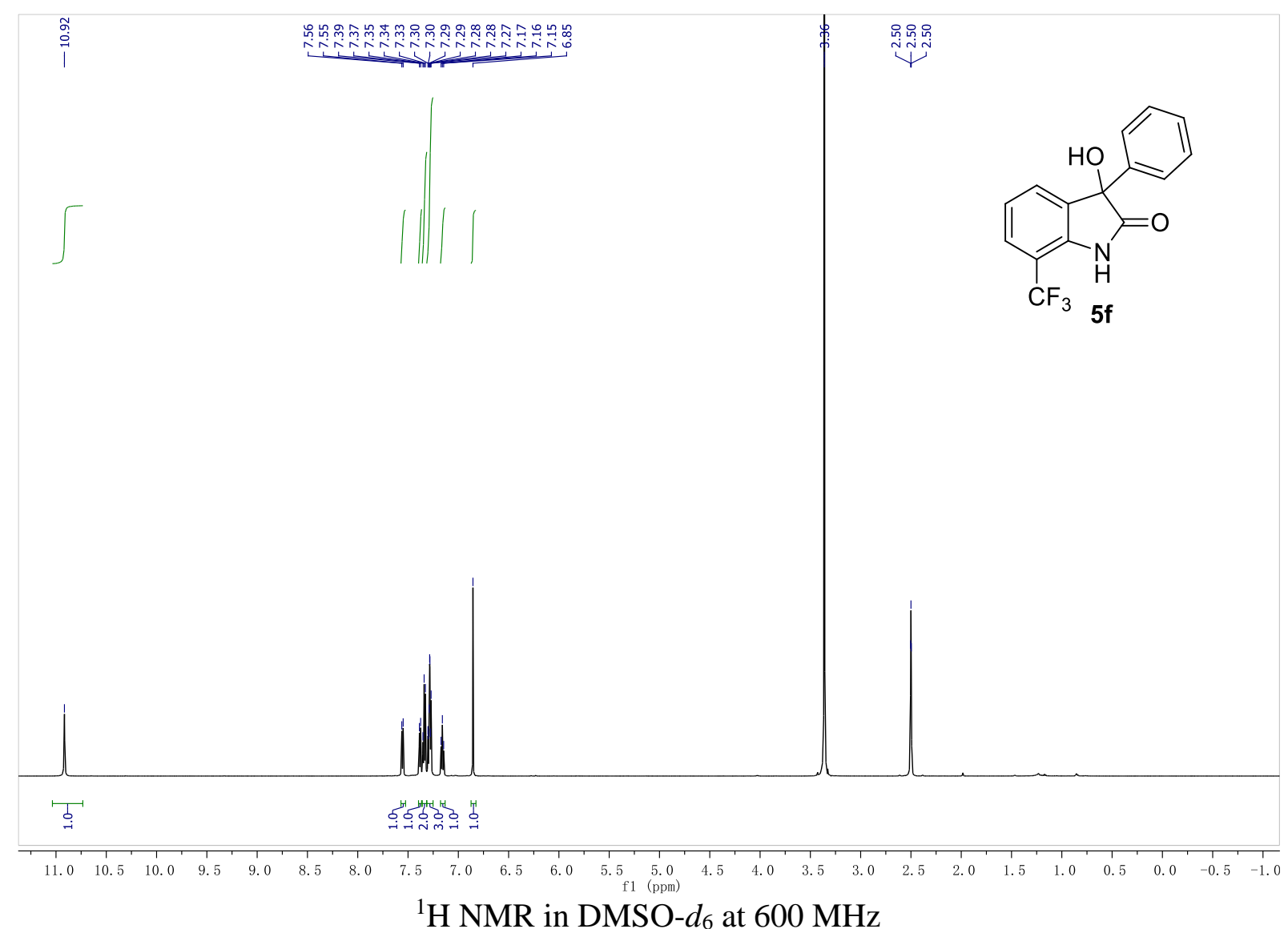

${ }^{1} \mathrm{H}$ NMR in DMSO- $d_{6}$ at $600 \mathrm{MHz}$

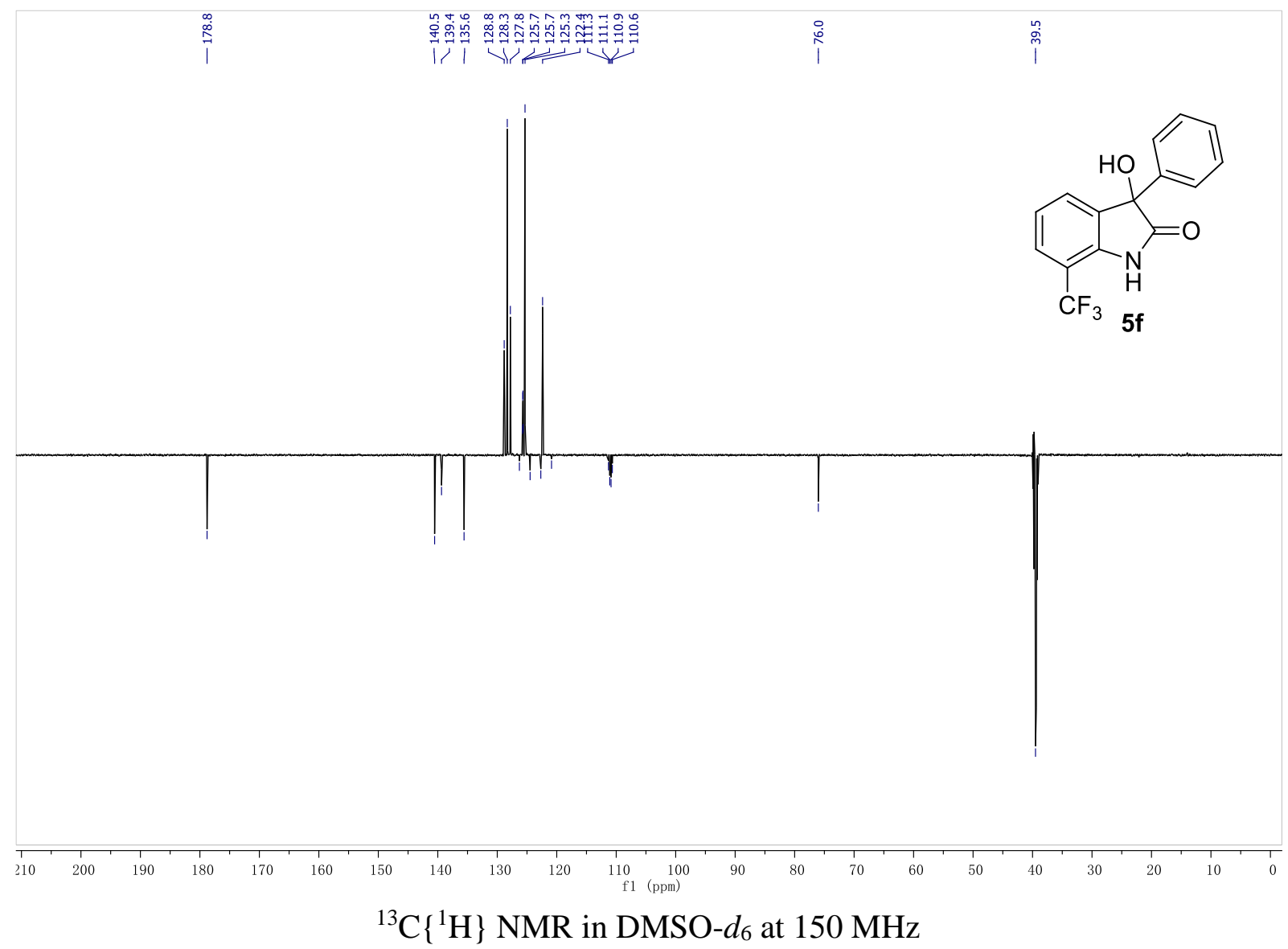




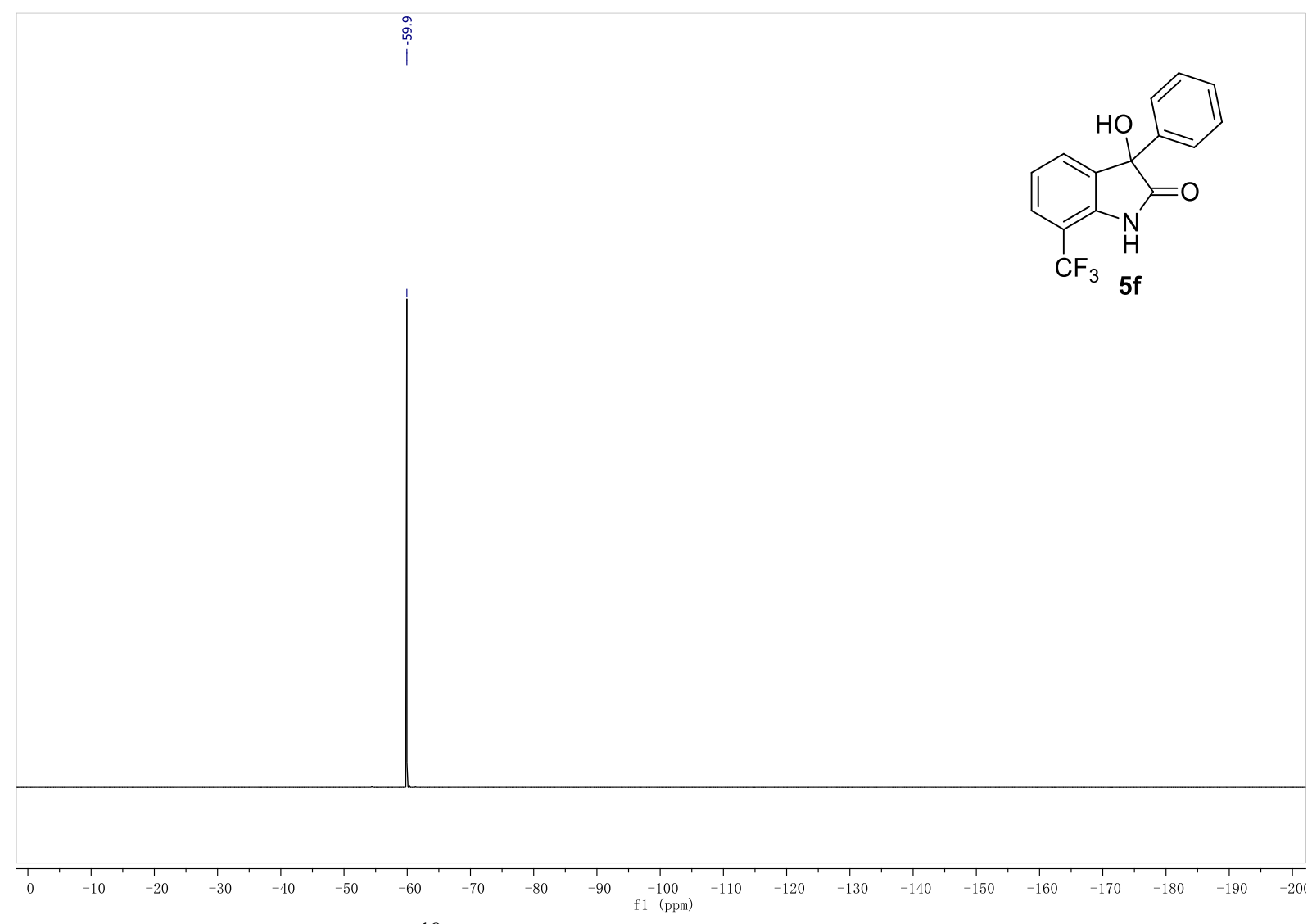

${ }^{19} \mathrm{~F}$ NMR in DMSO- $d_{6}$ at $565 \mathrm{MHz}$ 


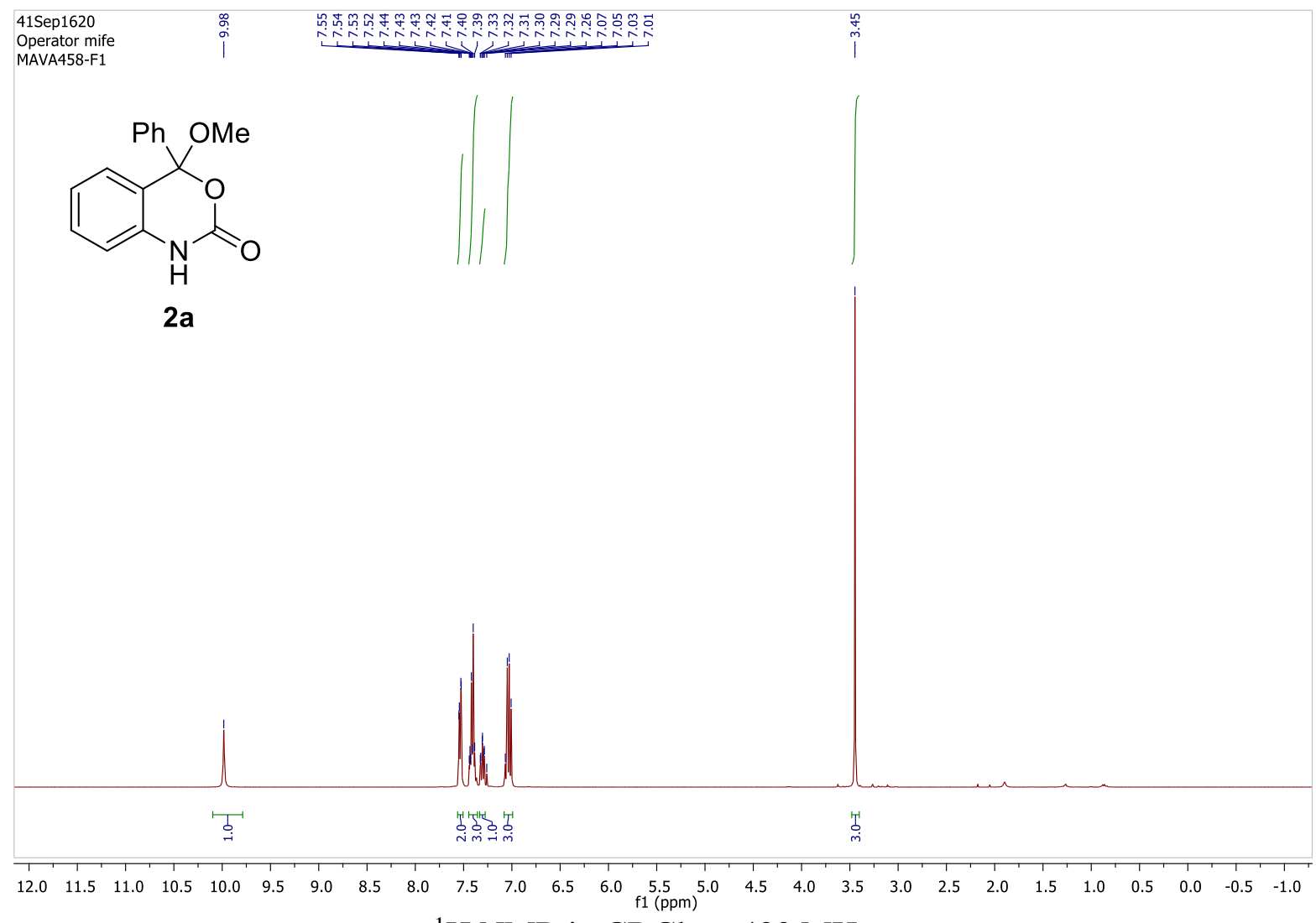

${ }^{1} \mathrm{H} \mathrm{NMR}$ in $\mathrm{CDCl}_{3}$ at $400 \mathrm{MHz}$

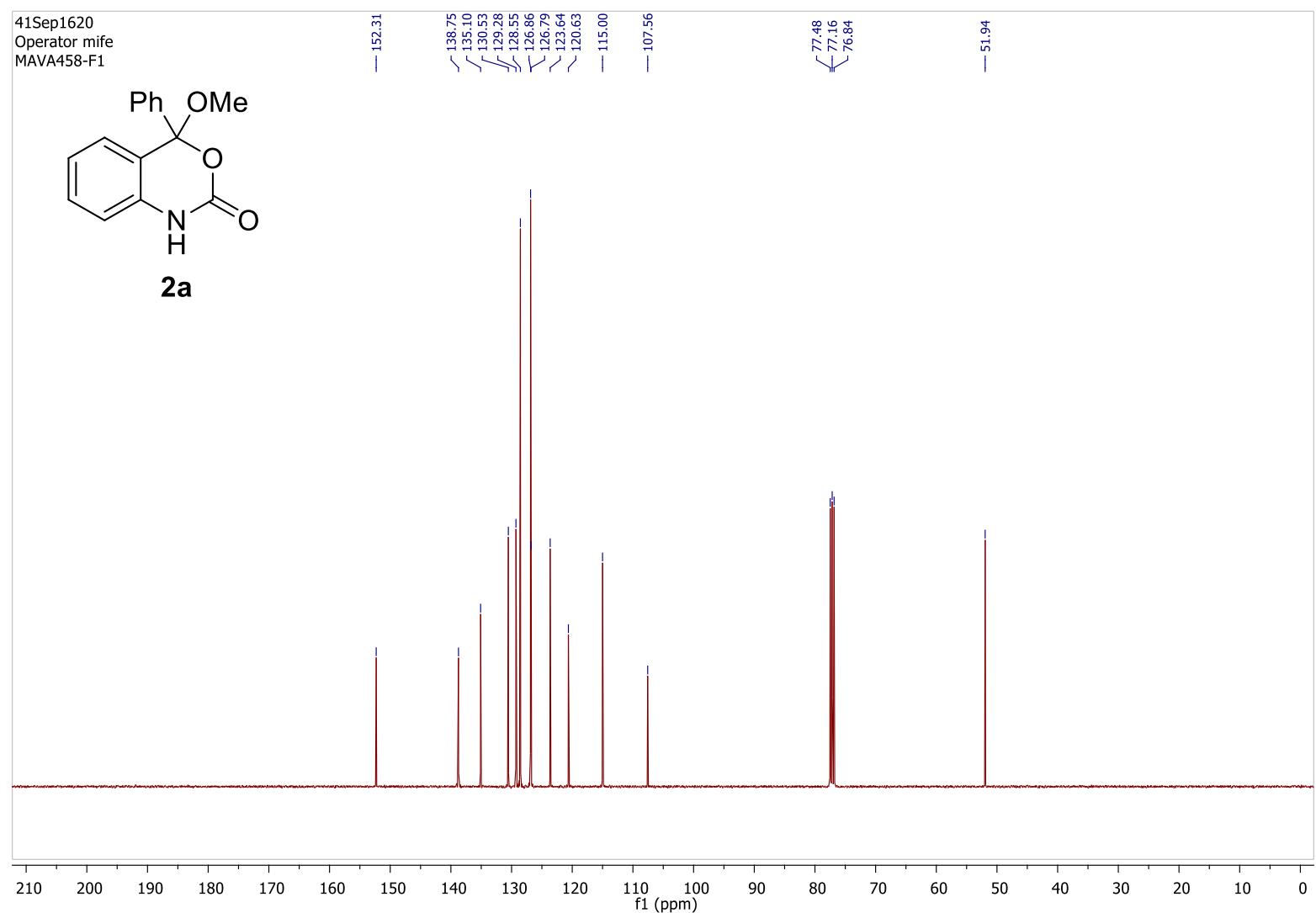

${ }^{13} \mathrm{C}\left\{{ }^{1} \mathrm{H}\right\}$ NMR in $\mathrm{CDCl}_{3}$ at $400 \mathrm{MHz}$ 
<smiles>CCOC1(c2ccccc2)OC(=O)Nc2ccccc21</smiles>
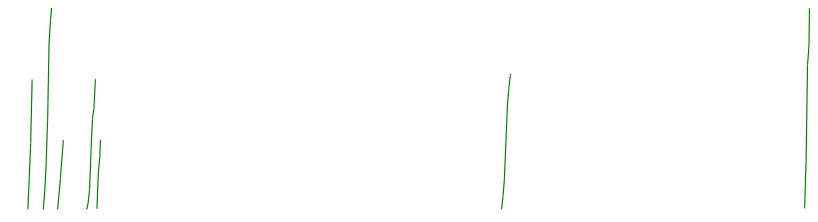

2b

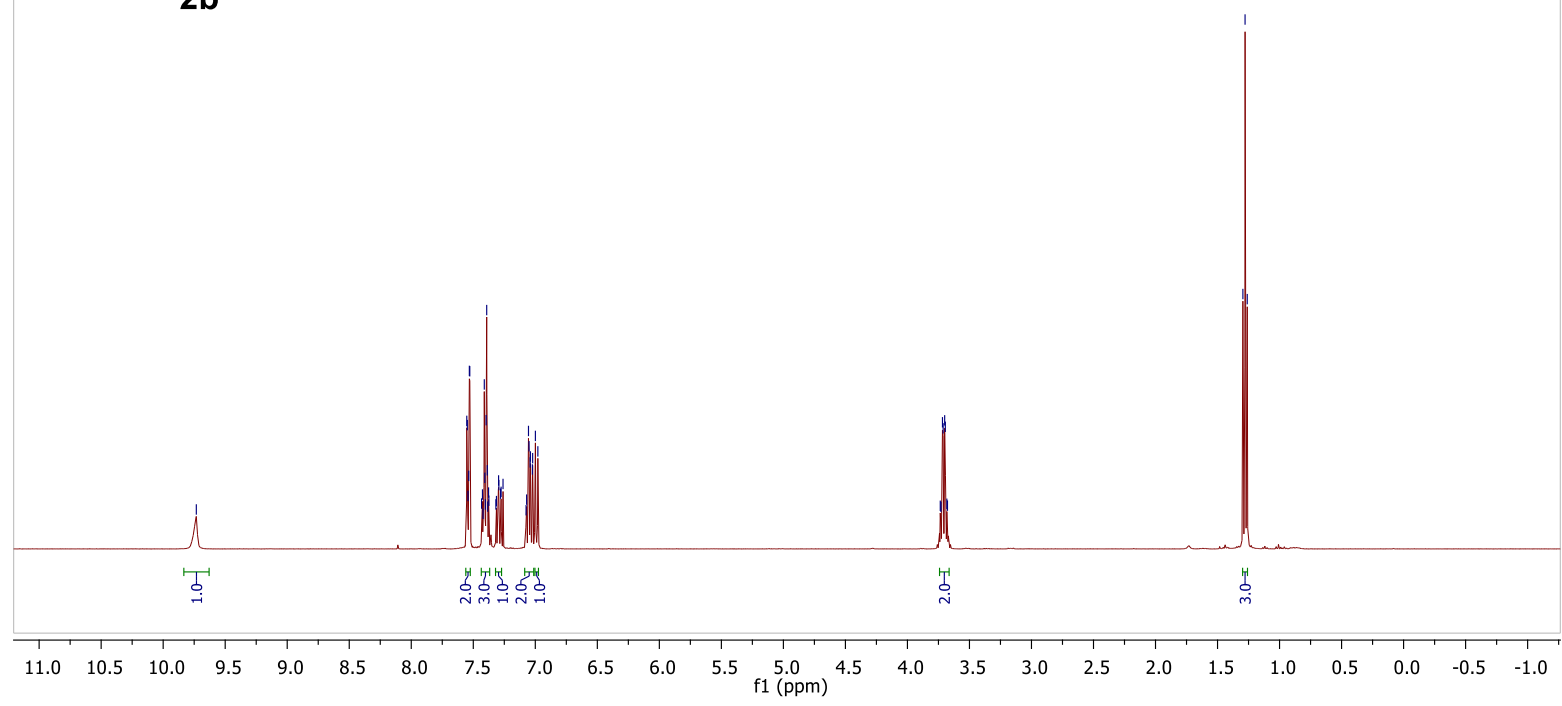

${ }^{1} \mathrm{H} \mathrm{NMR}$ in $\mathrm{CDCl}_{3}$ at $400 \mathrm{MHz}$

40ct1619

Operator mava

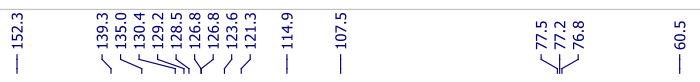

$\stackrel{\text { i }}{\stackrel{i}{7}}$<smiles>CCOC1(c2ccccc2)OC(=O)Nc2ccccc21</smiles>

2b

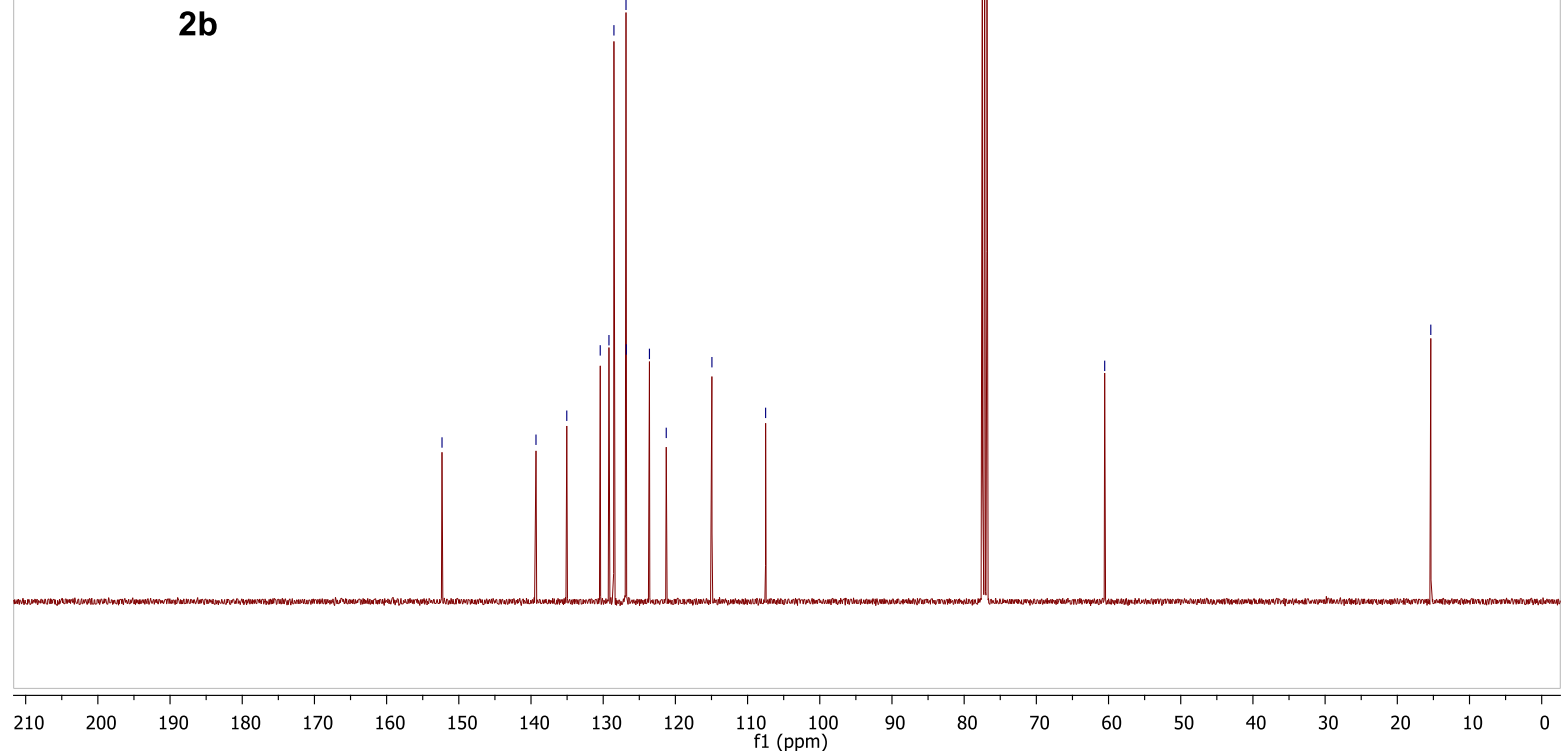

${ }^{13} \mathrm{C}\left\{{ }^{1} \mathrm{H}\right\} \mathrm{NMR}$ in $\mathrm{CDCl}_{3}$ at $100 \mathrm{MHz}$ 
<smiles>CCCOC1(c2ccccc2)OC(=O)Nc2ccccc21</smiles>

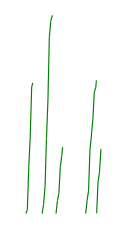

2c
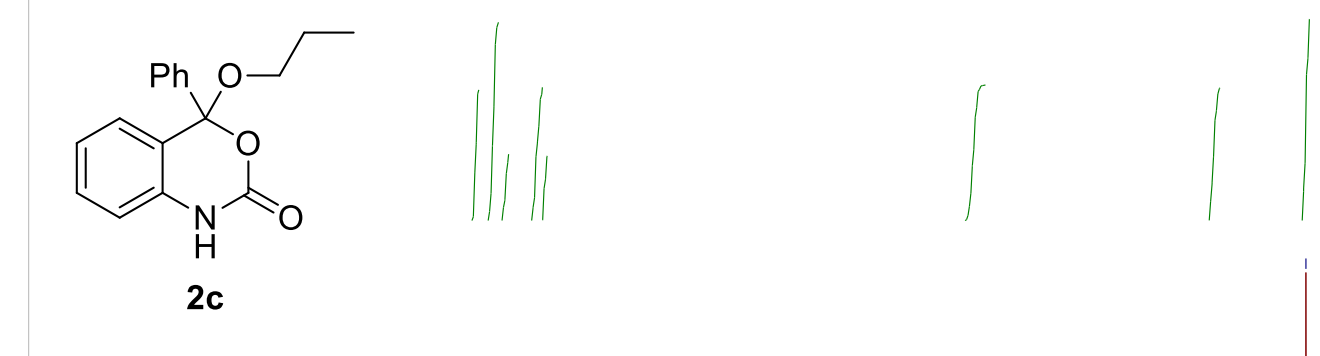<smiles>CCCOC1(c2ccccc2)OC(=O)Nc2ccccc21</smiles>

${ }^{1} \mathrm{H} \mathrm{NMR}$ in $\mathrm{CDCl}_{3}$ at $400 \mathrm{MHz}$

\section{Aug1319}

Operator mava

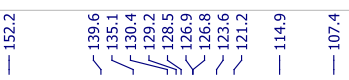

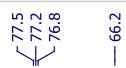

$\vec{i} \hat{i}$

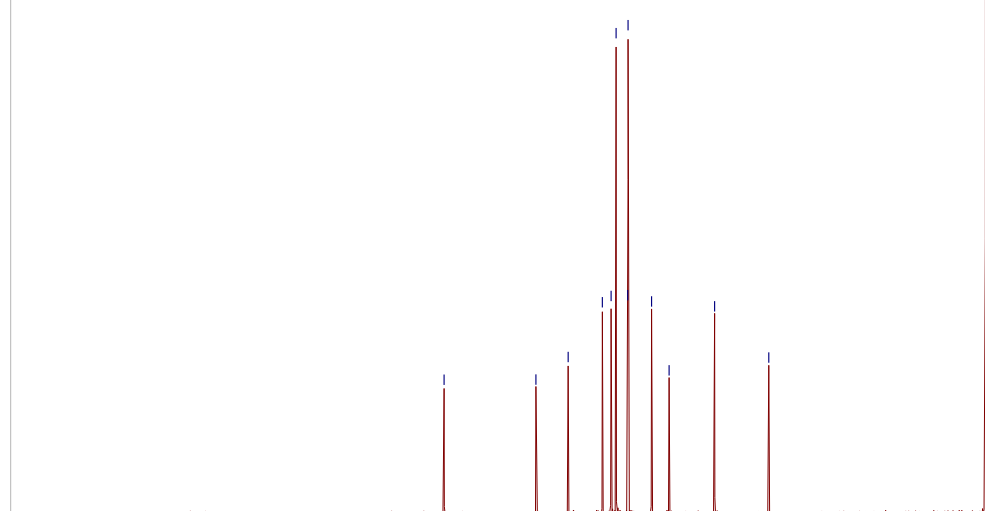

210

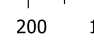

$190 \quad 180$
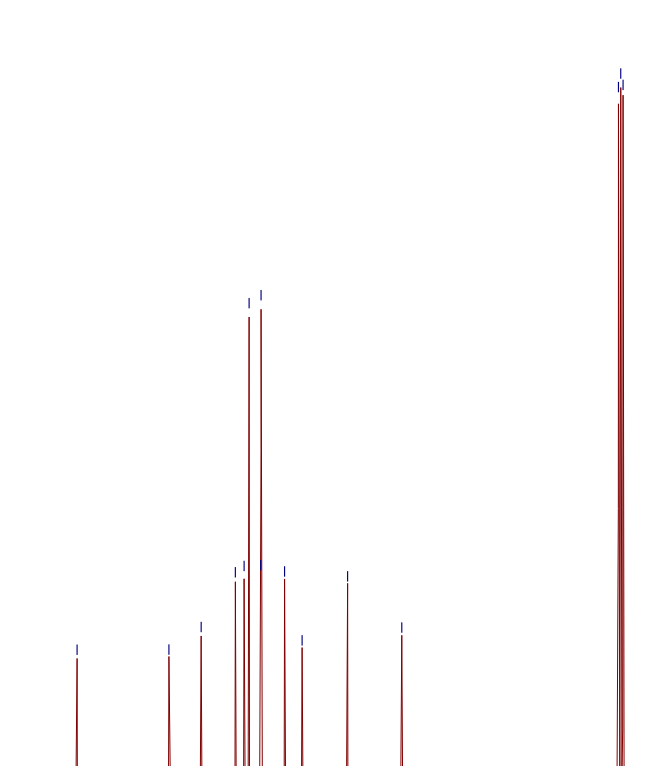

${ }^{13} \mathrm{C}\left\{{ }^{1} \mathrm{H}\right\} \mathrm{NMR}$ in $\mathrm{CDCl}_{3}$ at $100 \mathrm{MHz}$ 


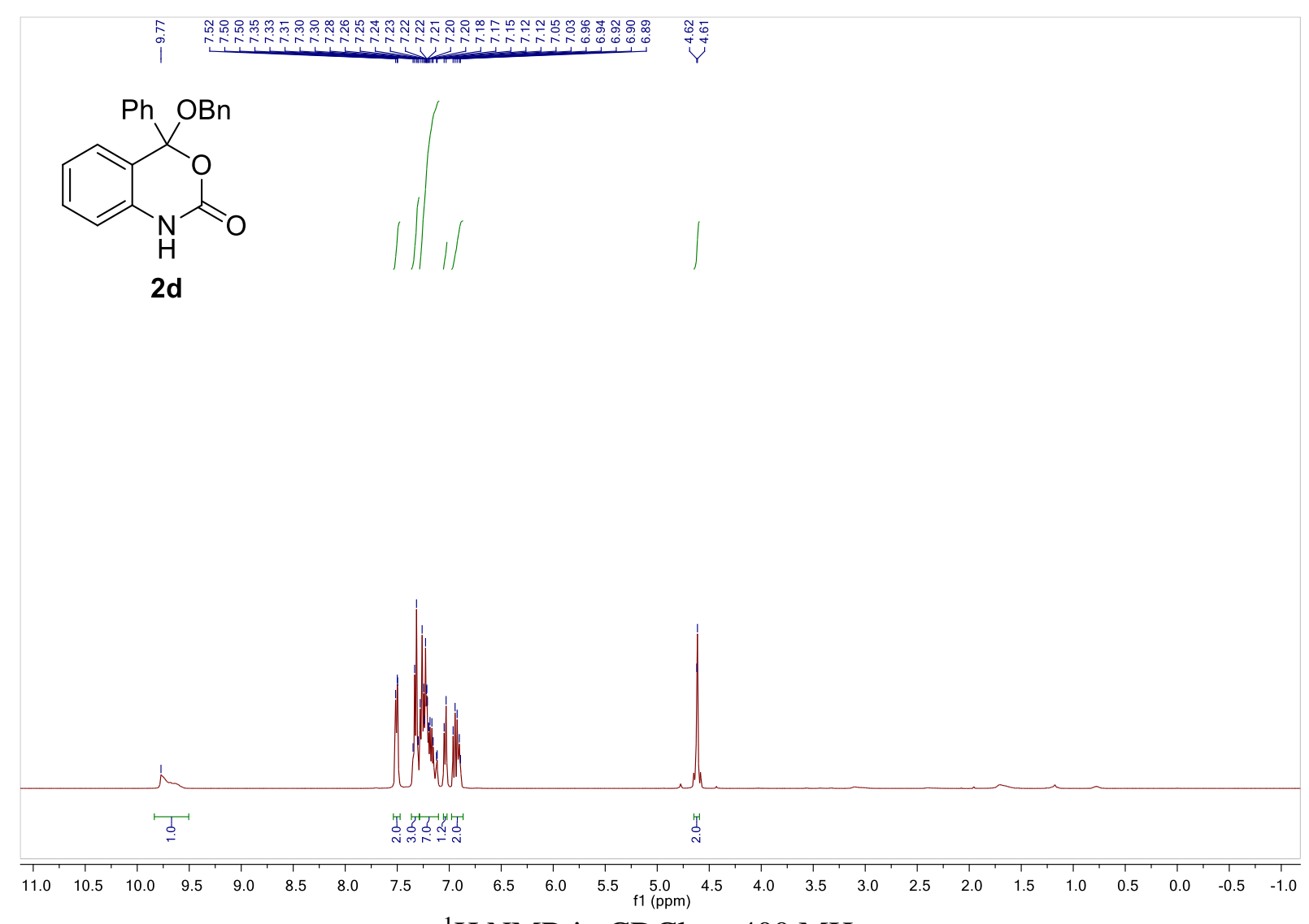

${ }^{1} \mathrm{H} \mathrm{NMR}$ in $\mathrm{CDCl}_{3}$ at $400 \mathrm{MHz}$

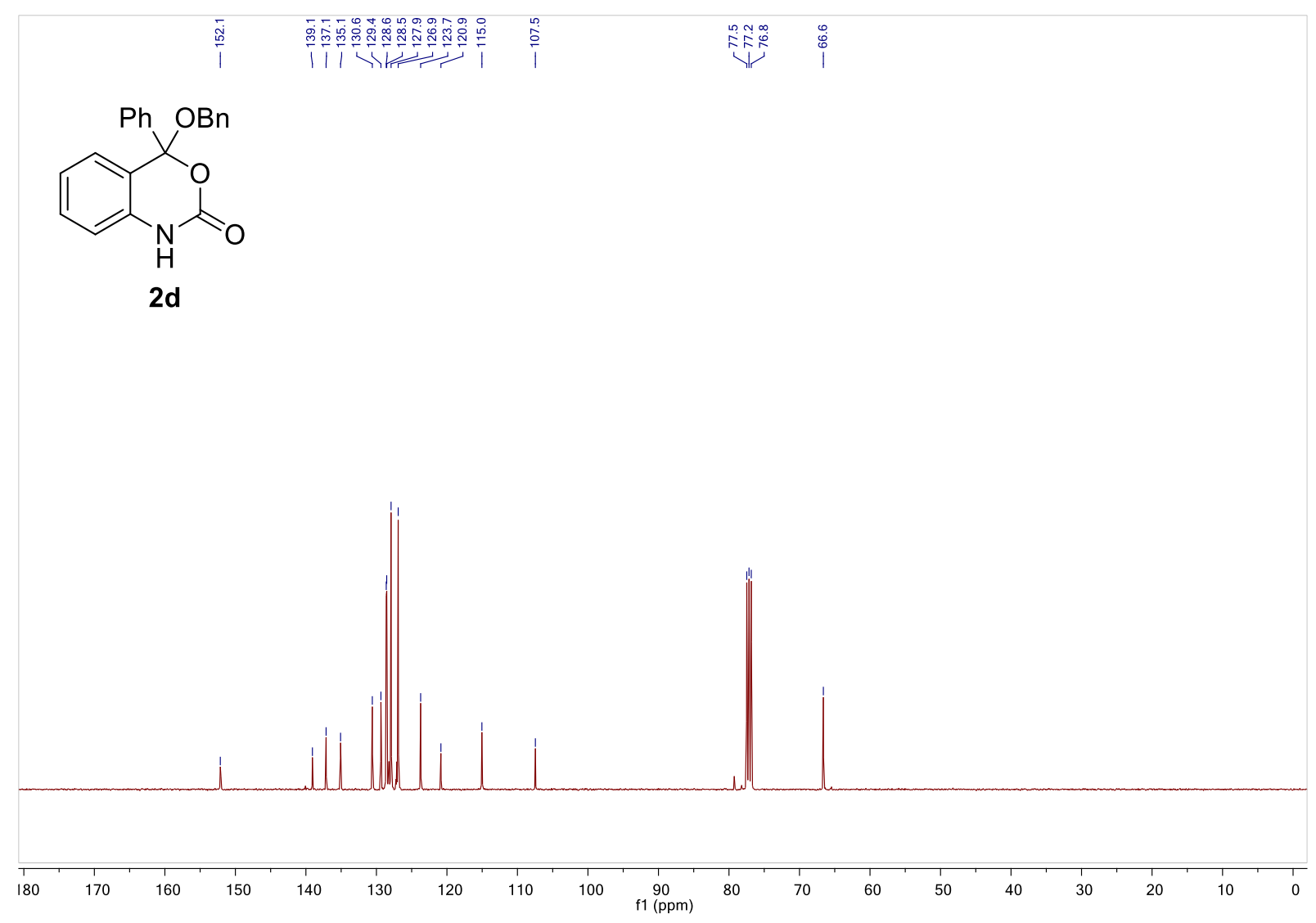

${ }^{13} \mathrm{C}\left\{{ }^{1} \mathrm{H}\right\}$ NMR in $\mathrm{CDCl}_{3}$ at $100 \mathrm{MHz}$ 


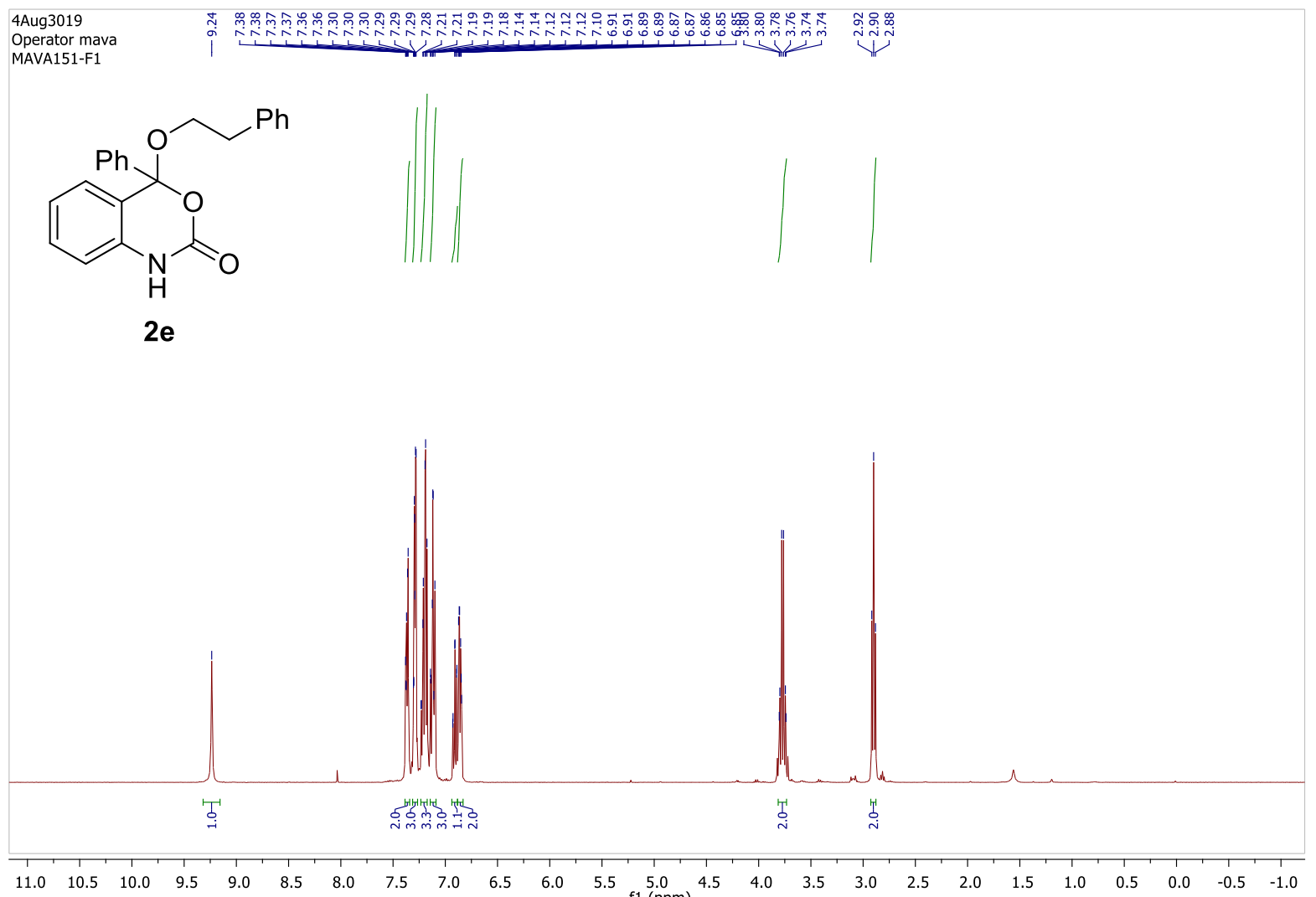

${ }^{1} \mathrm{H}$ NMR in $\mathrm{CDCl}_{3}$ at $400 \mathrm{MHz}$

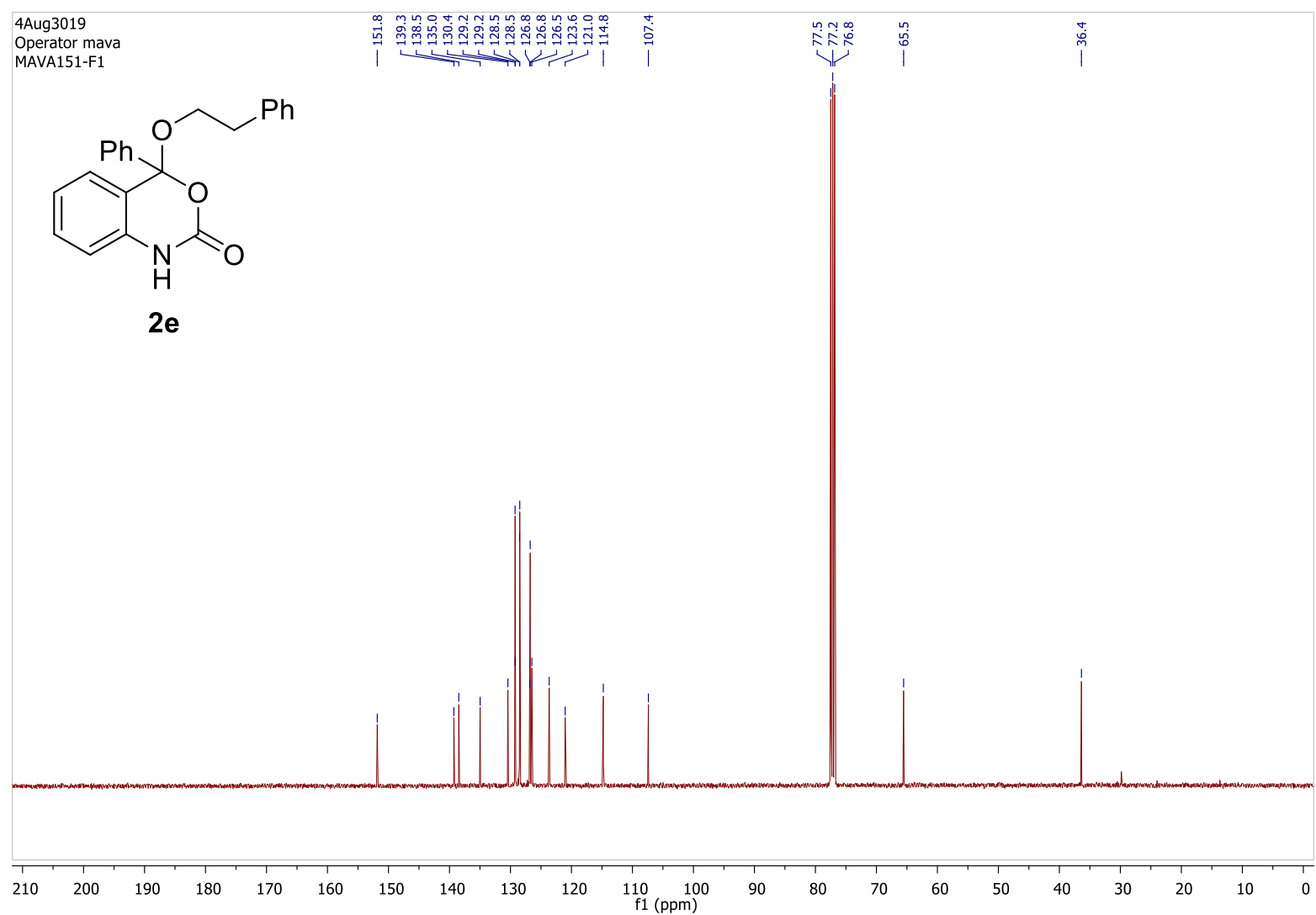

${ }^{13} \mathrm{C}\left\{{ }^{1} \mathrm{H}\right\} \mathrm{NMR}$ in $\mathrm{CDCl}_{3}$ at $100 \mathrm{MHz}$ 


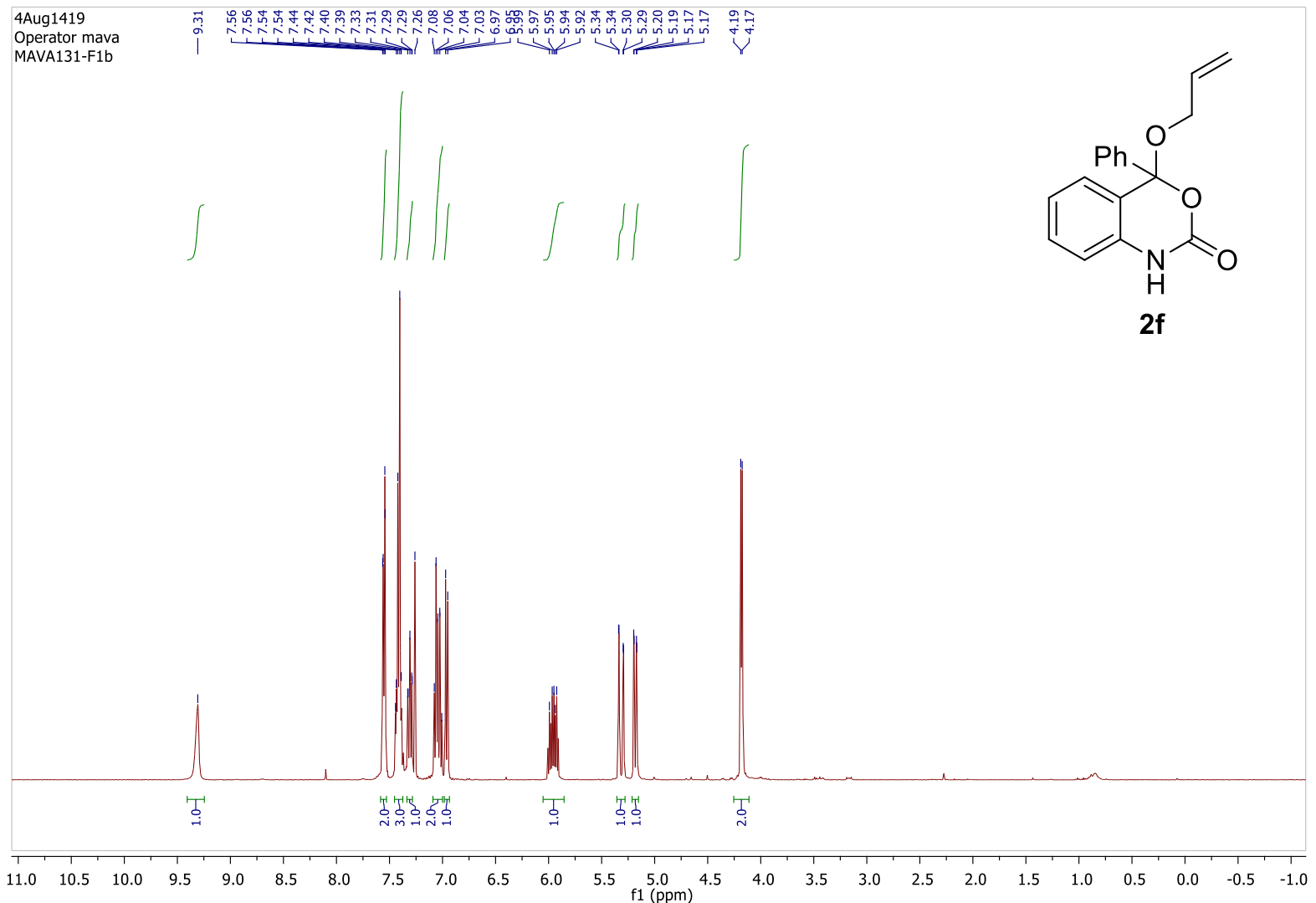

${ }^{1} \mathrm{H} \mathrm{NMR}$ in $\mathrm{CDCl}_{3}$ at $400 \mathrm{MHz}$

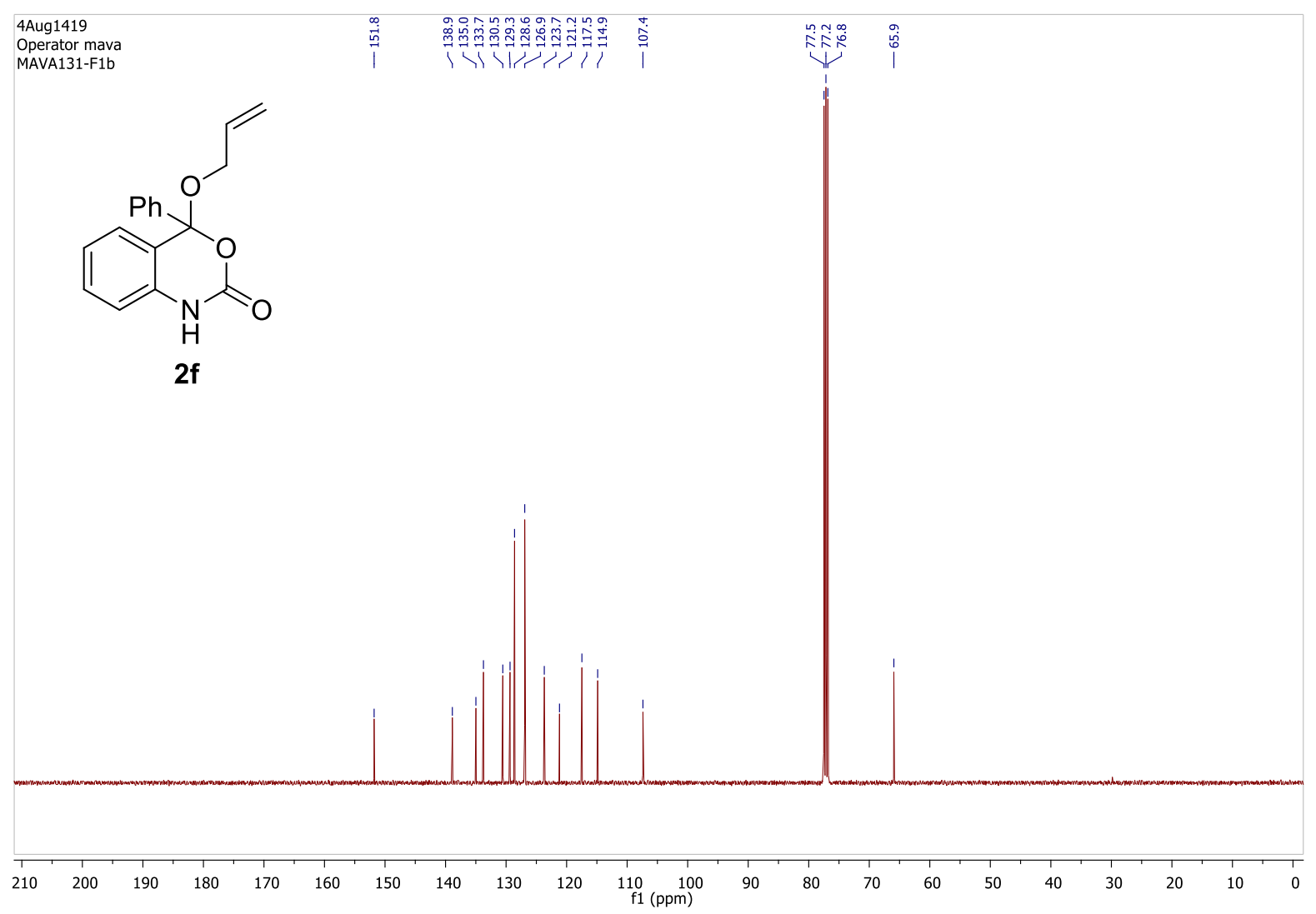

${ }^{13} \mathrm{C}\left\{{ }^{1} \mathrm{H}\right\}$ NMR in $\mathrm{CDCl}_{3}$ at $100 \mathrm{MHz}$ 


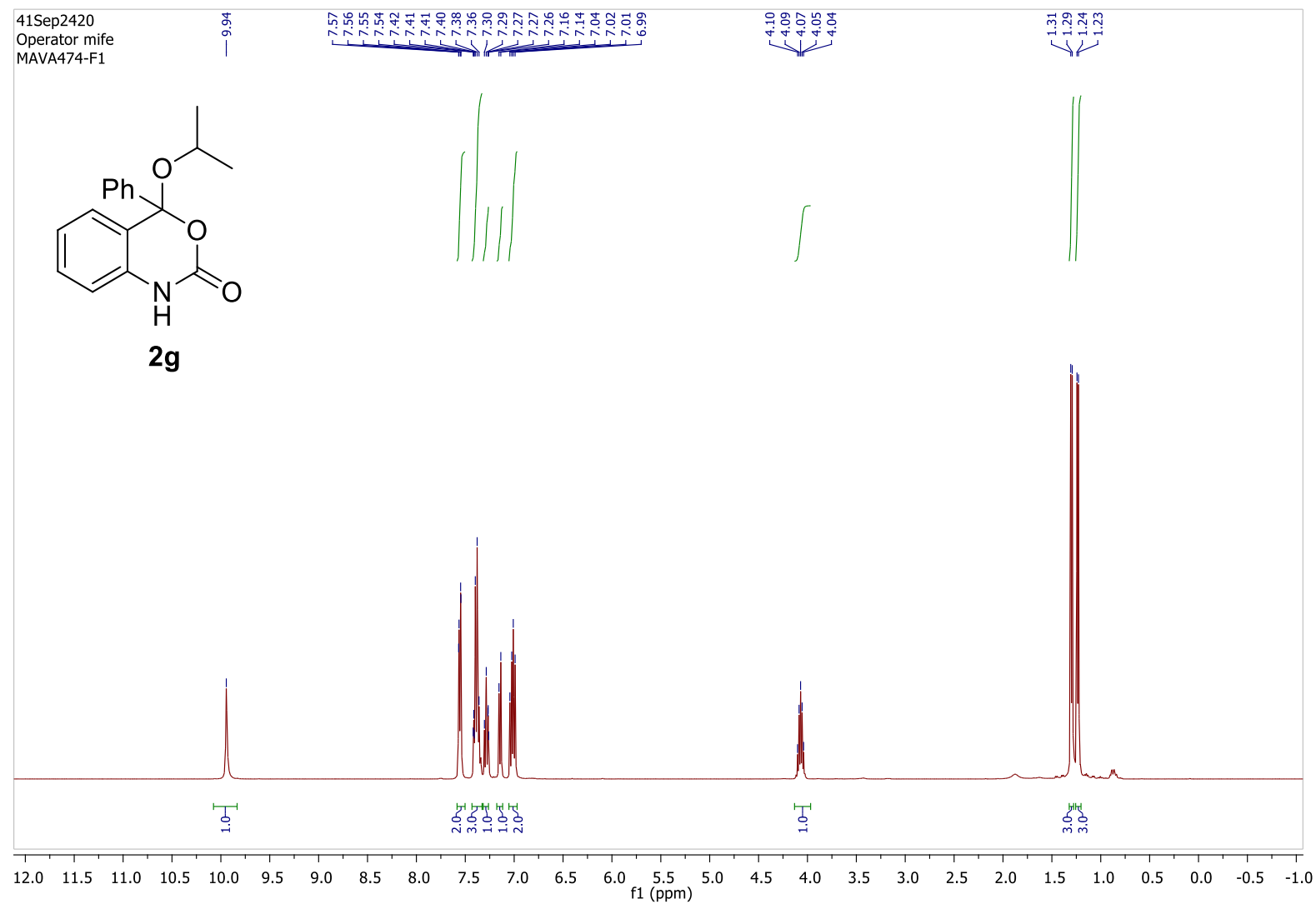

${ }^{1} \mathrm{H} \mathrm{NMR}$ in $\mathrm{CDCl}_{3}$ at $400 \mathrm{MHz}$

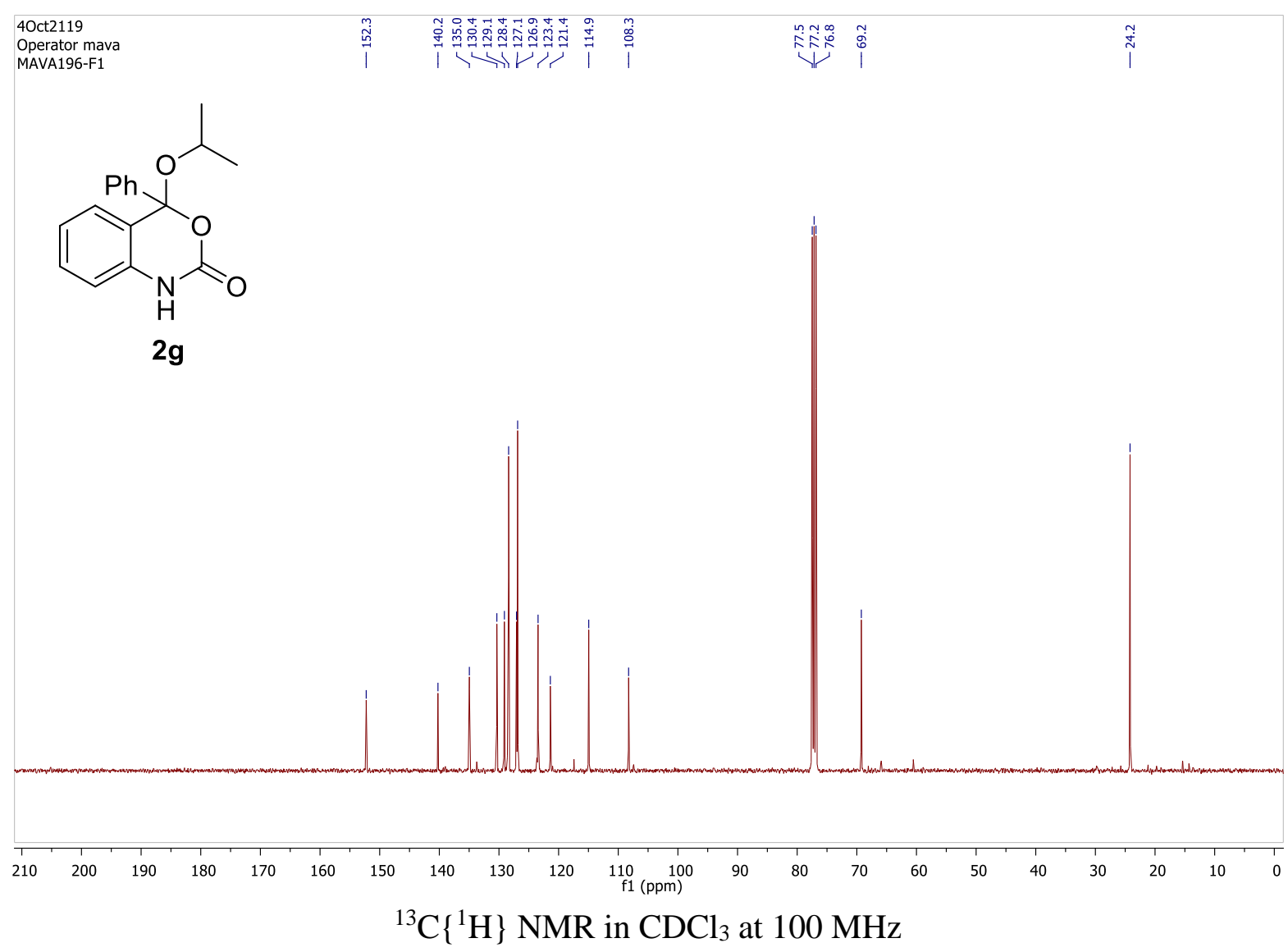




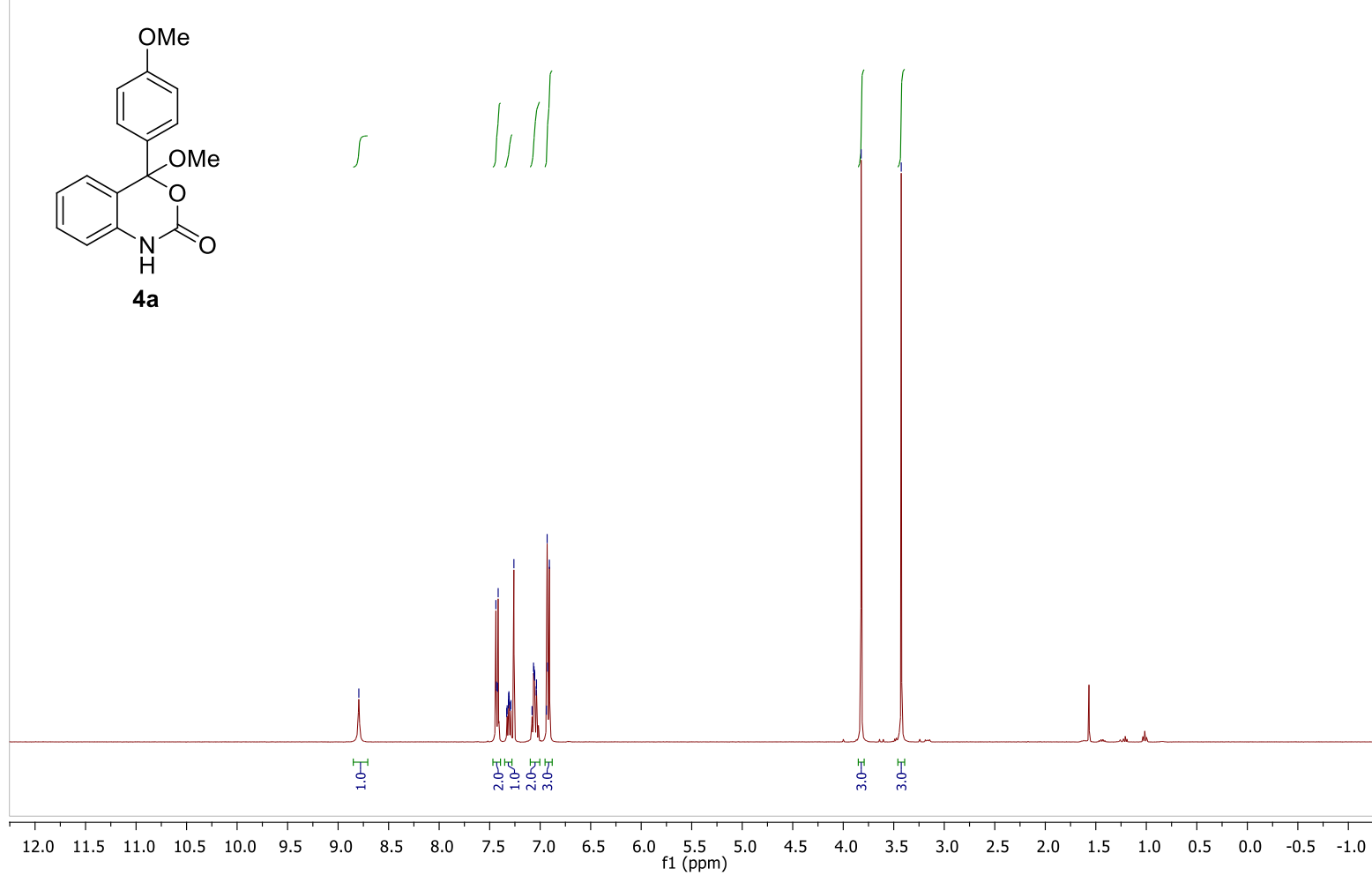

${ }^{1} \mathrm{H} \mathrm{NMR}$ in $\mathrm{CDCl}_{3}$ at $400 \mathrm{MHz}$

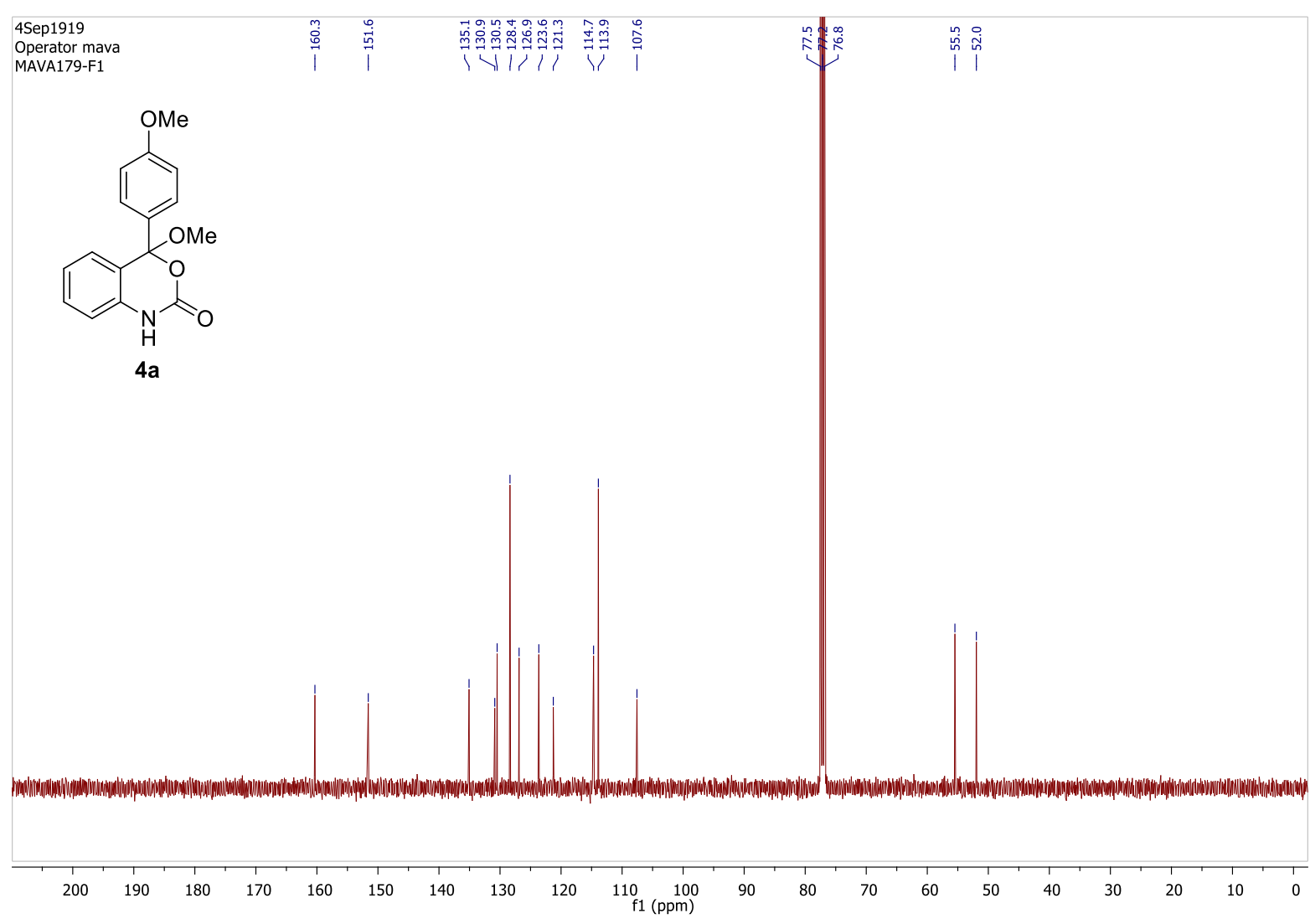

${ }^{13} \mathrm{C}\left\{{ }^{1} \mathrm{H}\right\} \mathrm{NMR}$ in $\mathrm{CDCl}_{3}$ at $100 \mathrm{MHz}$ 


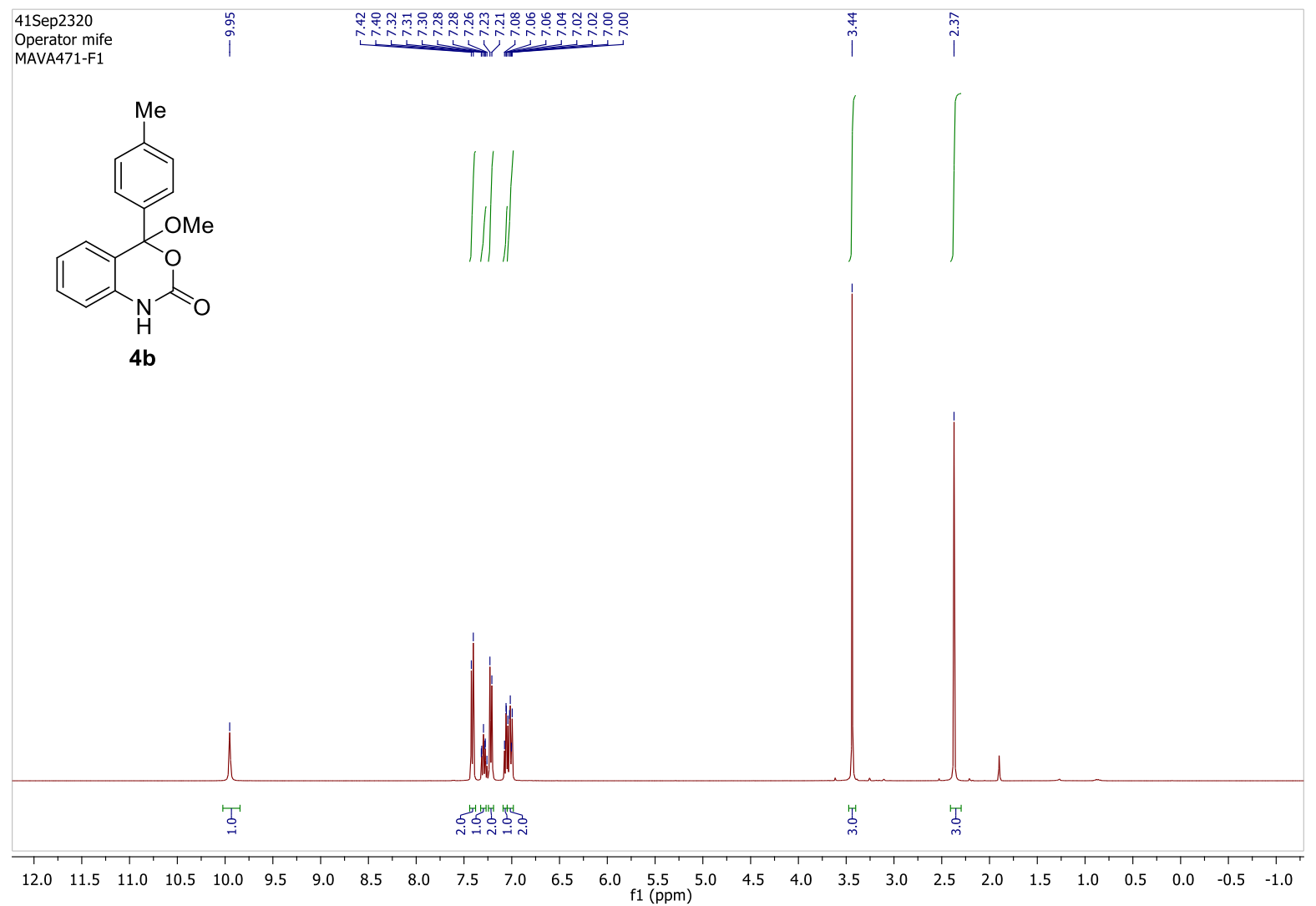

${ }^{1} \mathrm{H} \mathrm{NMR}$ in $\mathrm{CDCl}_{3}$ at $400 \mathrm{MHz}$

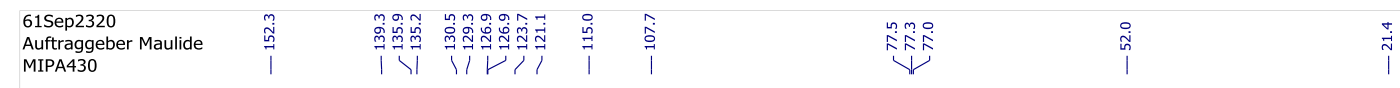<smiles>COC1(c2ccc(C)cc2)OC(=O)Nc2ccccc21</smiles>
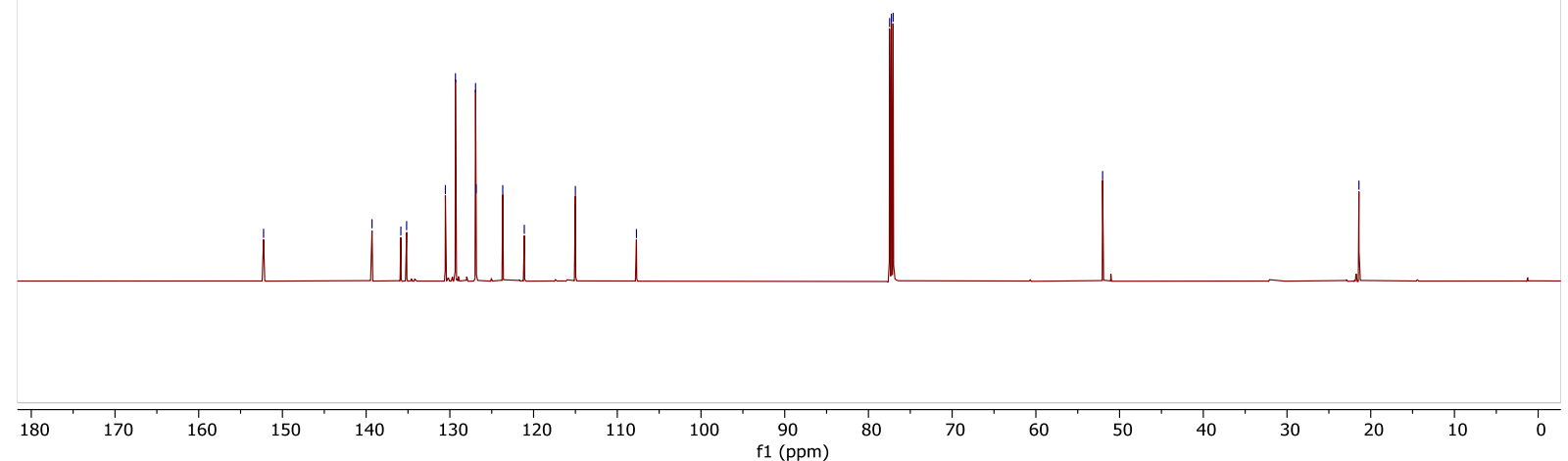

${ }^{13} \mathrm{C}\left\{{ }^{1} \mathrm{H}\right\} \mathrm{NMR}$ in $\mathrm{CDCl}_{3}$ at $150 \mathrm{MHz}$ 


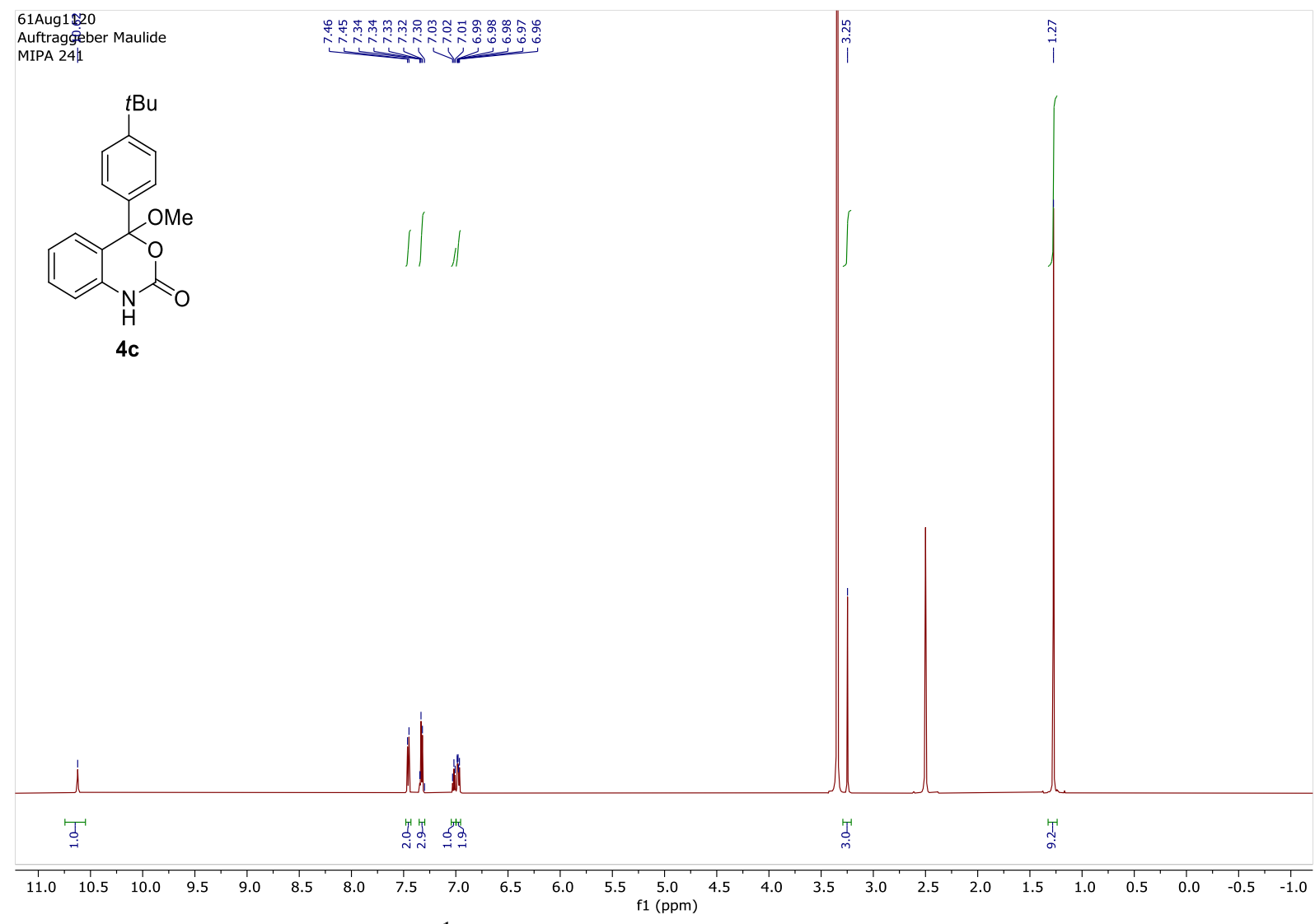

${ }^{1} \mathrm{H} \mathrm{NMR} \mathrm{in} \mathrm{CDCl}_{3}$ at $600 \mathrm{MHz}$

61Aug1120.122.1.1r
Auftraggeber Maulide
MIPA 241

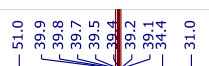<smiles>COC1(c2ccc(C(C)(C)C)cc2)OC(=O)Nc2ccccc21</smiles>
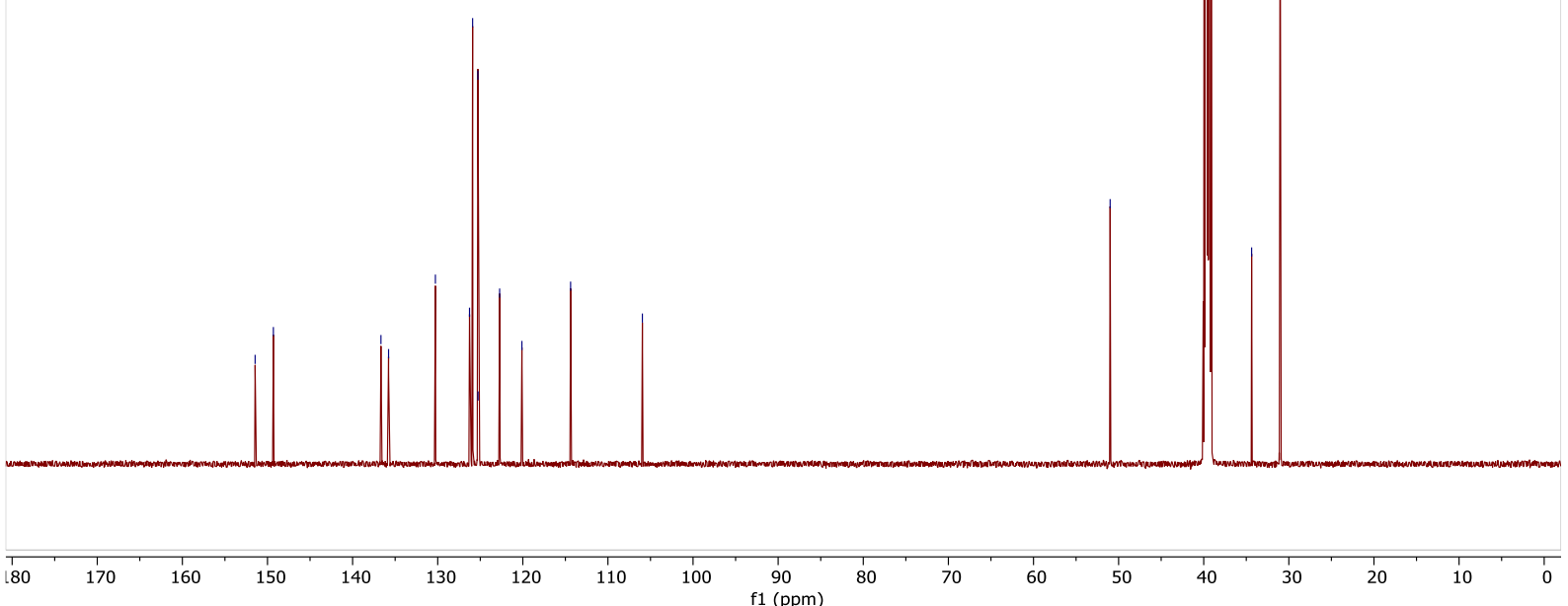

${ }^{13} \mathrm{C}\left\{{ }^{1} \mathrm{H}\right\} \mathrm{NMR}$ in $\mathrm{CDCl}_{3}$ at $150 \mathrm{MHz}$ 

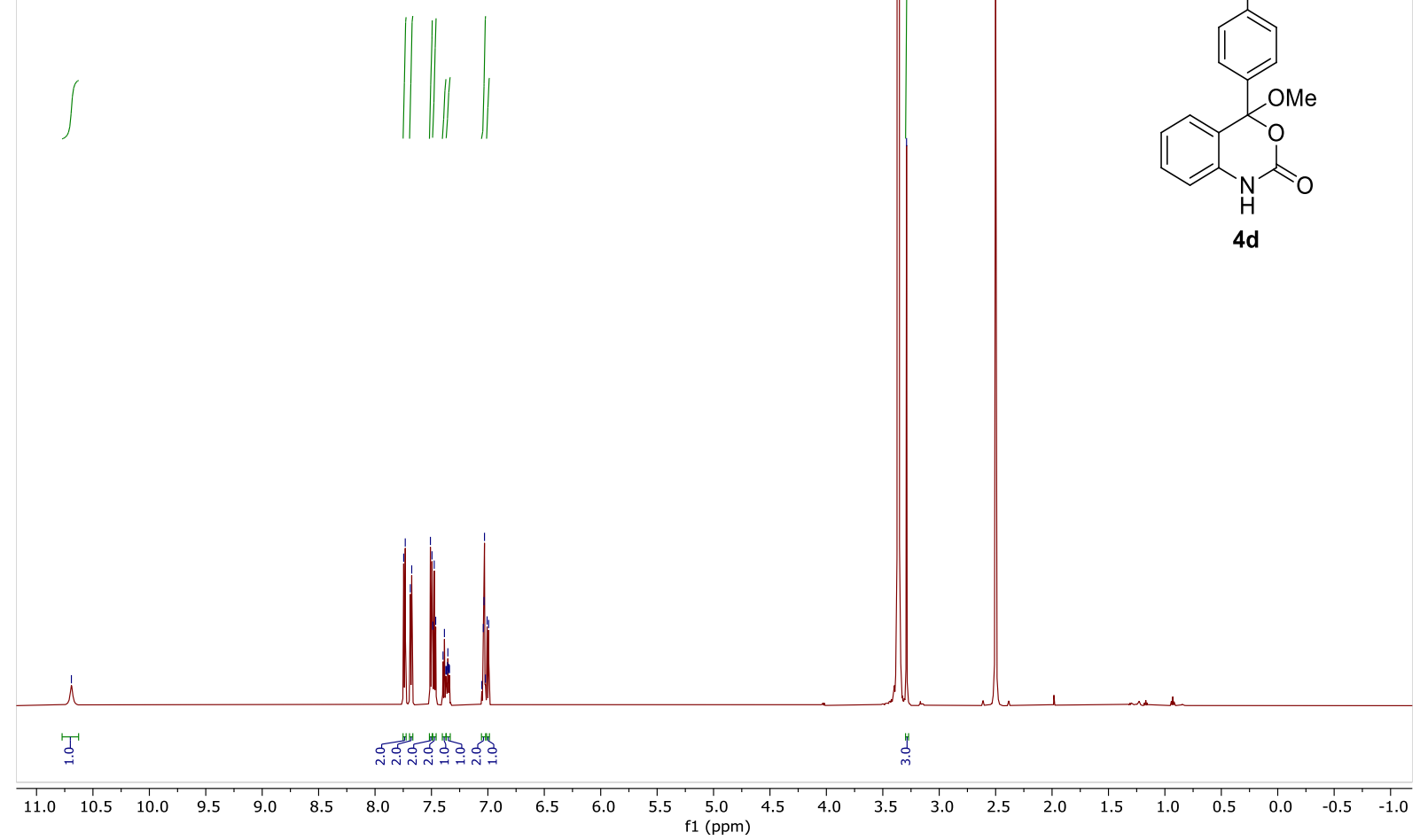

${ }^{1} \mathrm{H} \mathrm{NMR}$ in $\mathrm{CDCl}_{3}$ at $600 \mathrm{MHz}$

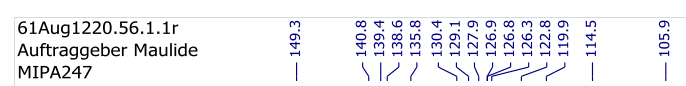<smiles>COC1(c2ccc(-c3ccccc3)cc2)C(=O)Nc2ccccc21</smiles>

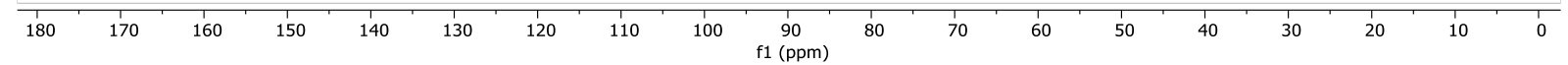

${ }^{13} \mathrm{C}\left\{{ }^{1} \mathrm{H}\right\} \mathrm{NMR}$ in $\mathrm{CDCl}_{3}$ at $150 \mathrm{MHz}$ 


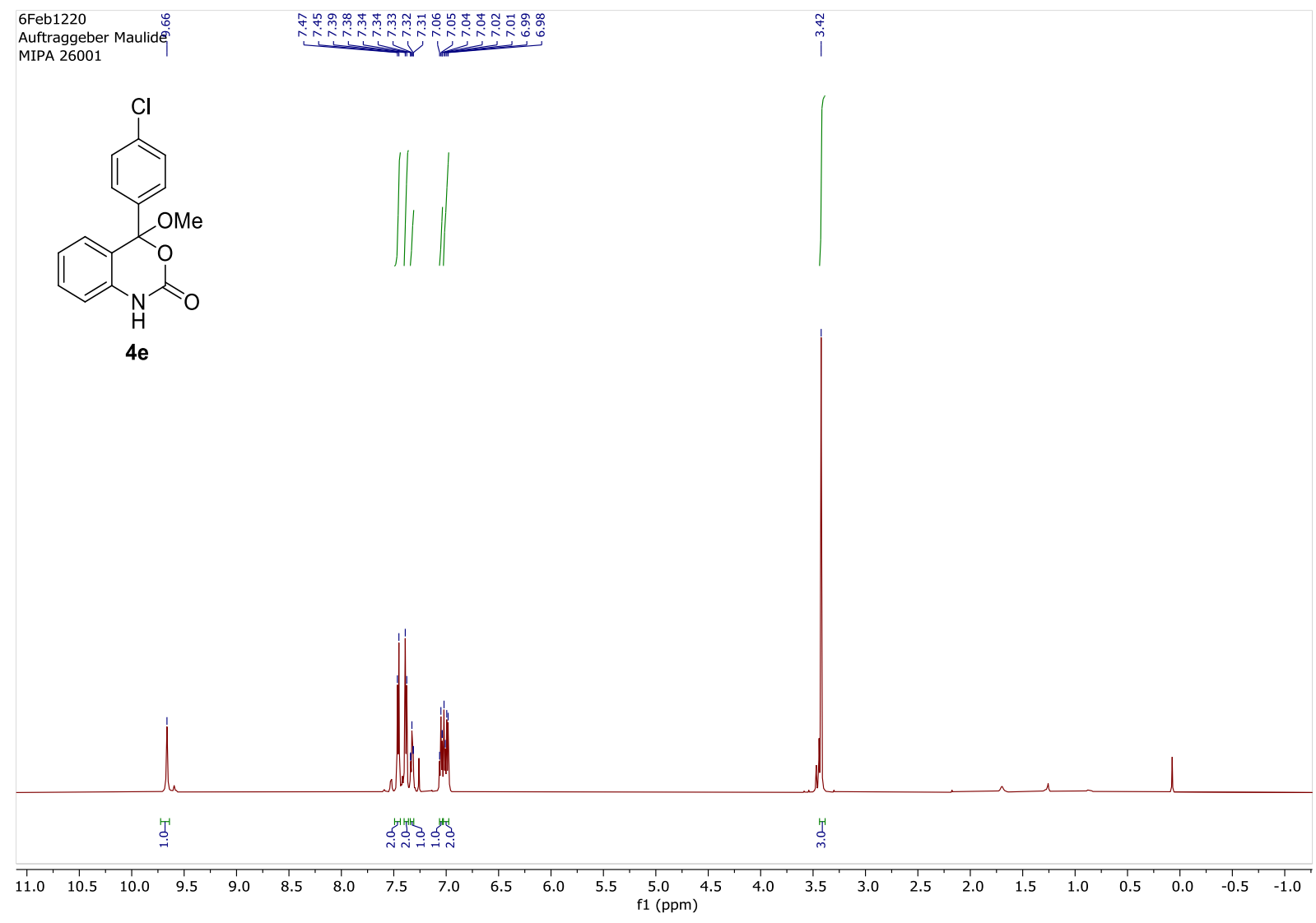

${ }^{1} \mathrm{H}$ NMR in $\mathrm{CDCl}_{3}$ at $600 \mathrm{MHz}$

6Feb1220
Auftraggeber Maulide
MIPA 26001

MIPA 26001<smiles>COC1(c2ccc(Cl)cc2)C(=O)Nc2ccccc21</smiles>

$4 e$

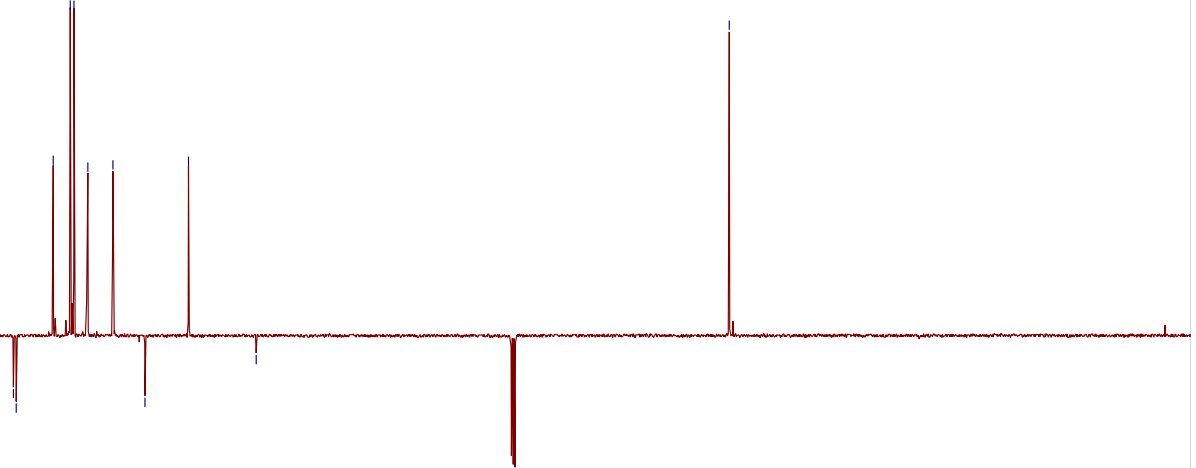

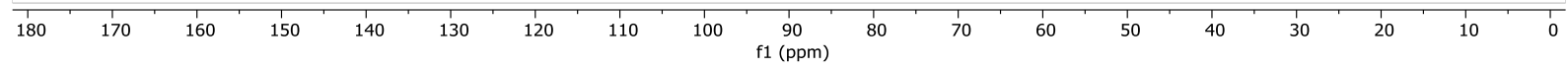

${ }^{13} \mathrm{C}\left\{{ }^{1} \mathrm{H}\right\} \mathrm{NMR}$ in $\mathrm{CDCl}_{3}$ at $150 \mathrm{MHz}$ 


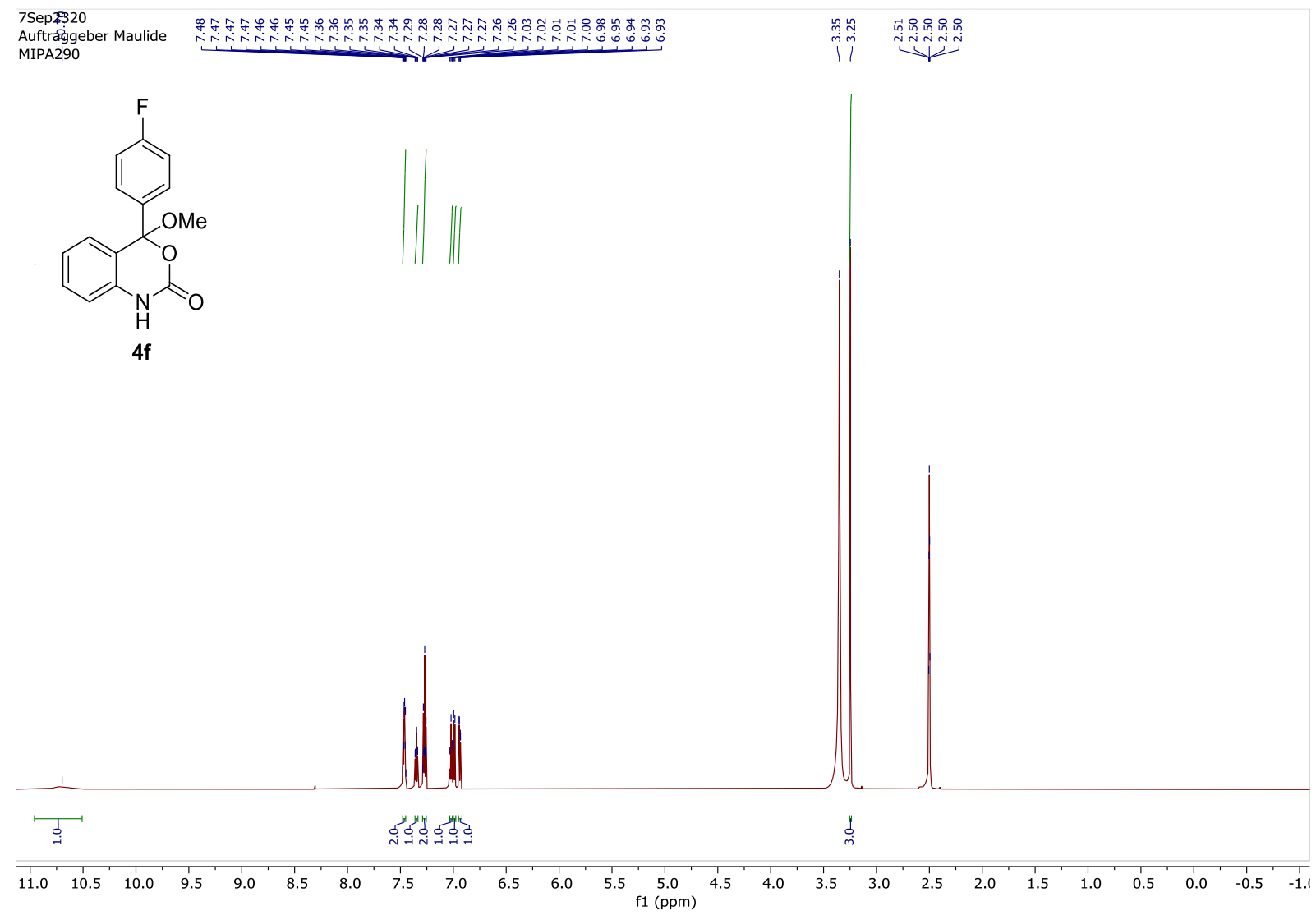

${ }^{1} \mathrm{H} \mathrm{NMR}$ in $\mathrm{CDCl}_{3}$ at $700 \mathrm{MHz}$

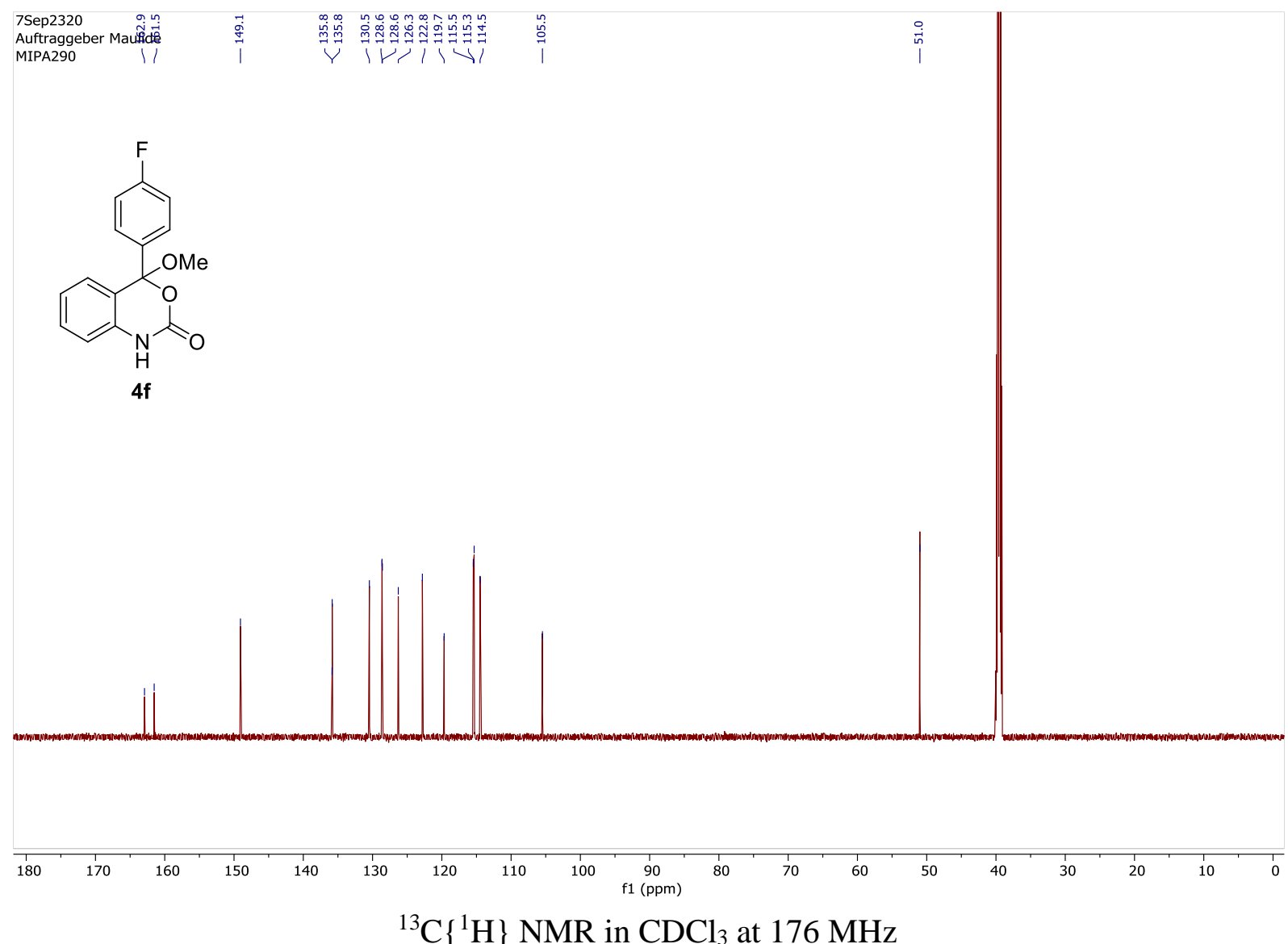

${ }^{13} \mathrm{C}\left\{{ }^{1} \mathrm{H}\right\}$ NMR in $\mathrm{CDCl}_{3}$ at $176 \mathrm{MHz}$ 
7 Sep 2320<smiles>COC1(c2ccc(F)cc2)OC(=O)Nc2ccccc21</smiles>

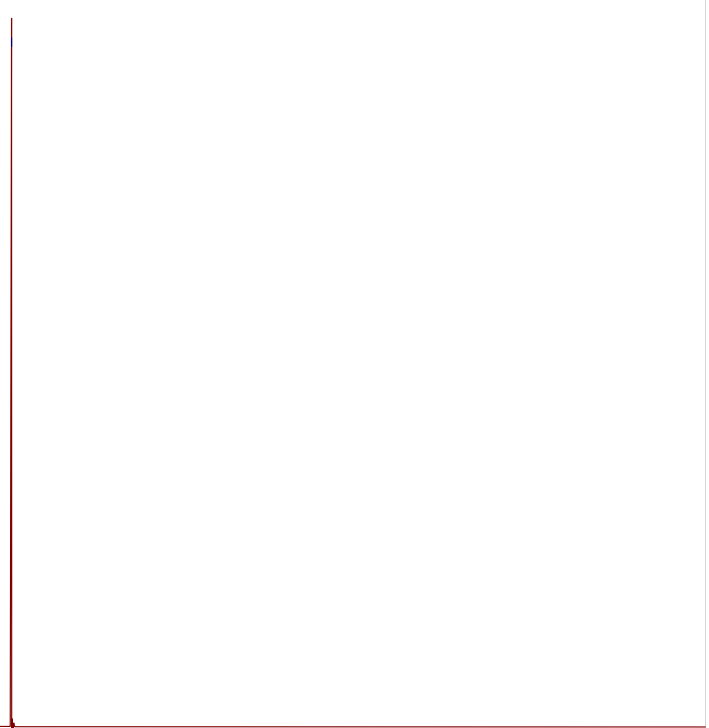

$$
\text { To }
$$

$\begin{array}{rrrrr}1 & -10 & -20 & -30 & -4\end{array}$

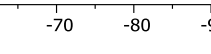

-100
-100

$\begin{array}{lll}120 & -130 & -140\end{array}$

\begin{tabular}{llllll}
\hline 150 & -160 & -170 & -180 & -190 & -200
\end{tabular}

${ }^{19} \mathrm{~F}$ NMR in DMSO- $d_{6}$ at $659 \mathrm{MHz}$ 


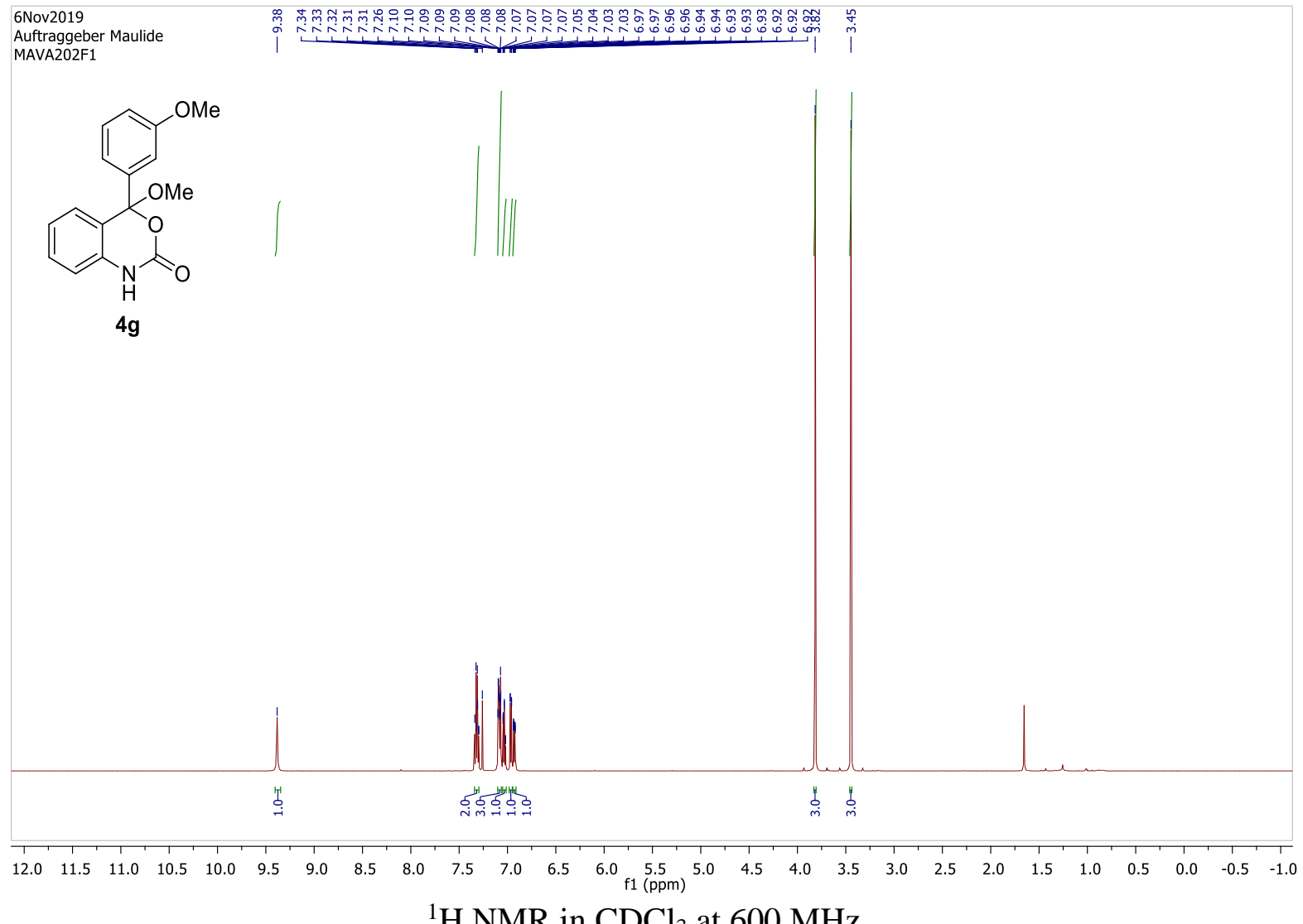

${ }^{1} \mathrm{H} \mathrm{NMR}$ in $\mathrm{CDCl}_{3}$ at $600 \mathrm{MHz}$

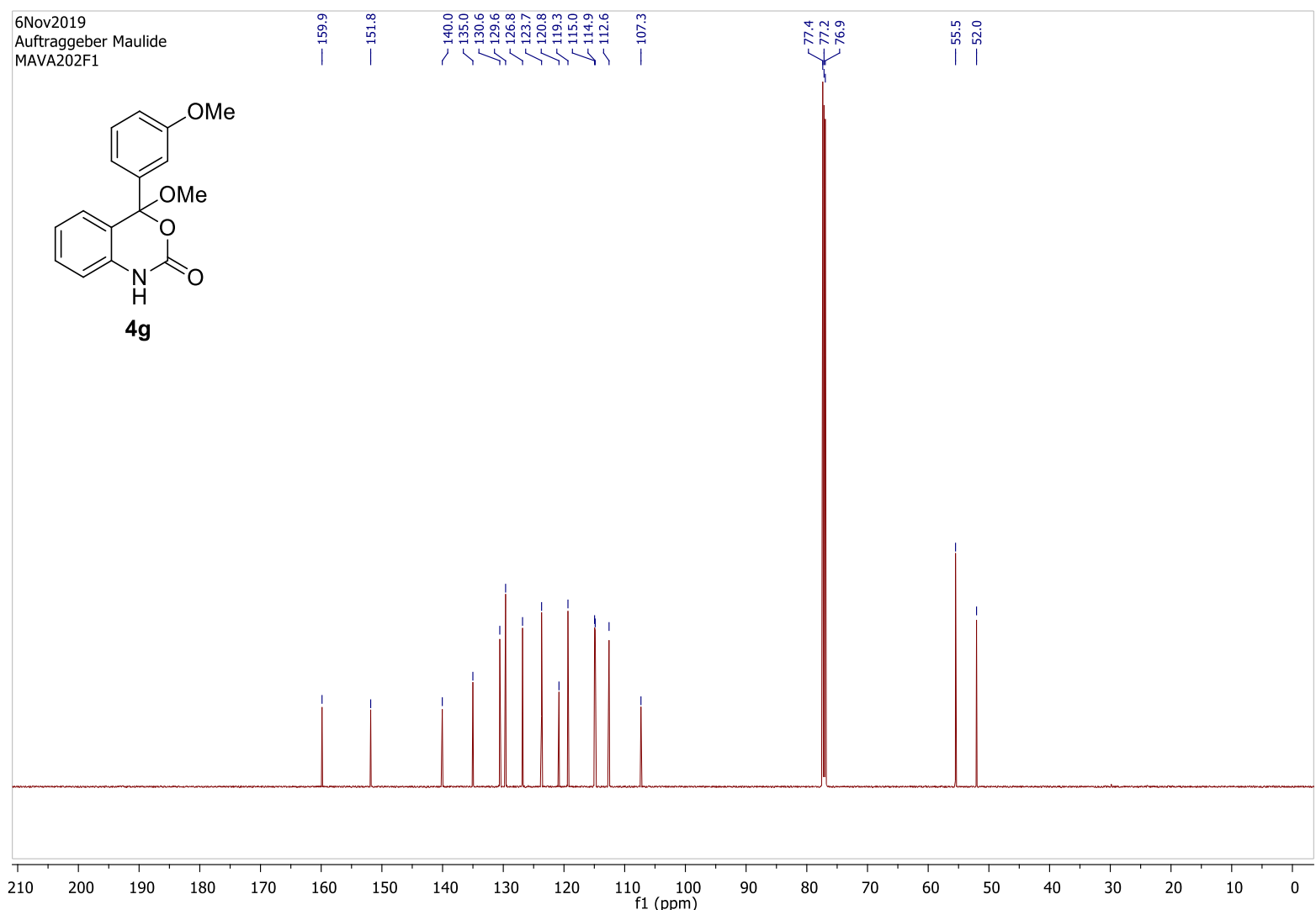

${ }^{13} \mathrm{C}\left\{{ }^{1} \mathrm{H}\right\} \mathrm{NMR}$ in $\mathrm{CDCl}_{3}$ at $150 \mathrm{MHz}$ 


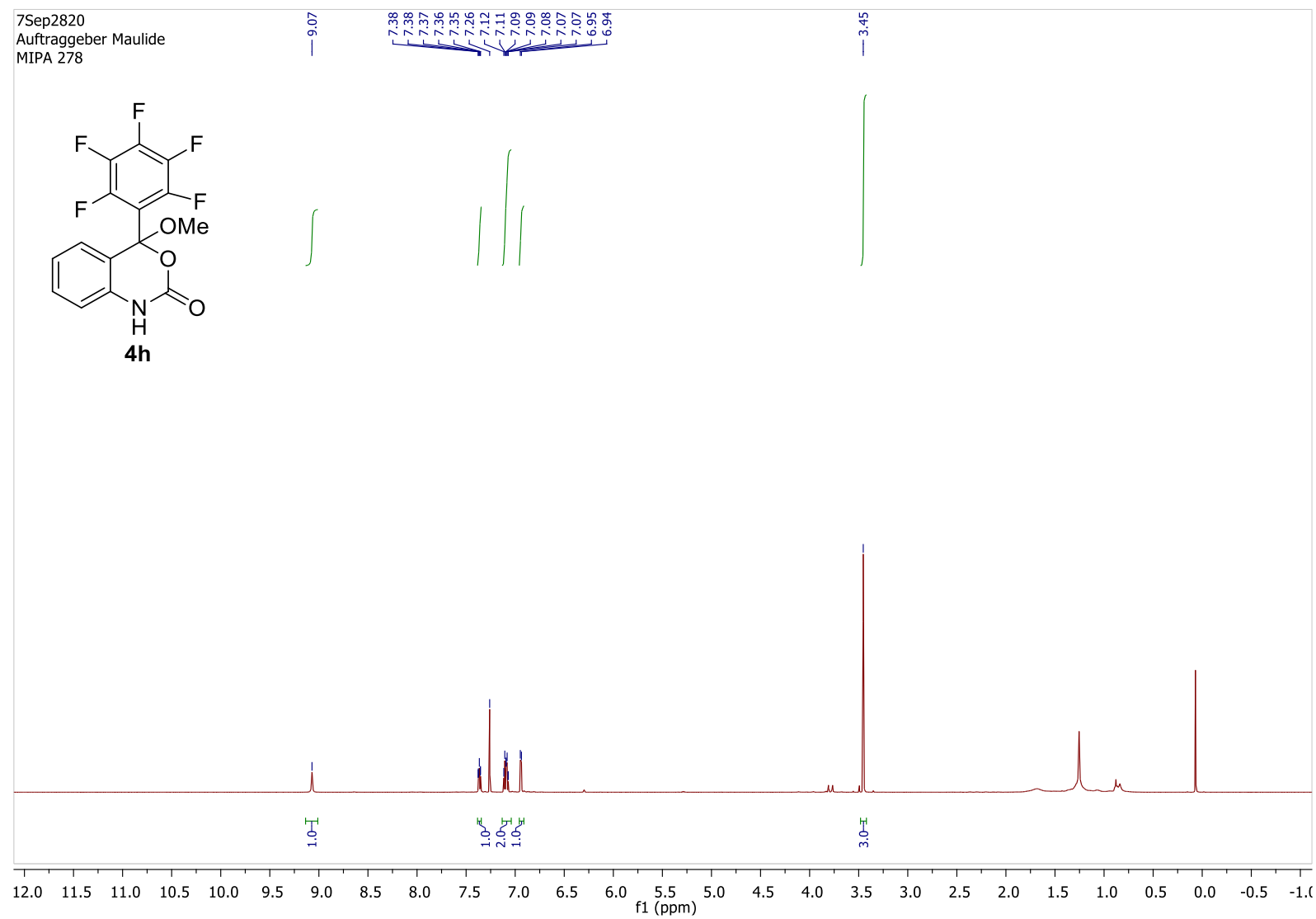

${ }^{1} \mathrm{H} \mathrm{NMR}$ in $\mathrm{CDCl}_{3}$ at $700 \mathrm{MHz}$

\section{Sep2820}

Auftraggeber Maulide

MIPA 278

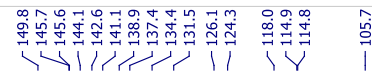

$\stackrel{\infty}{i}$

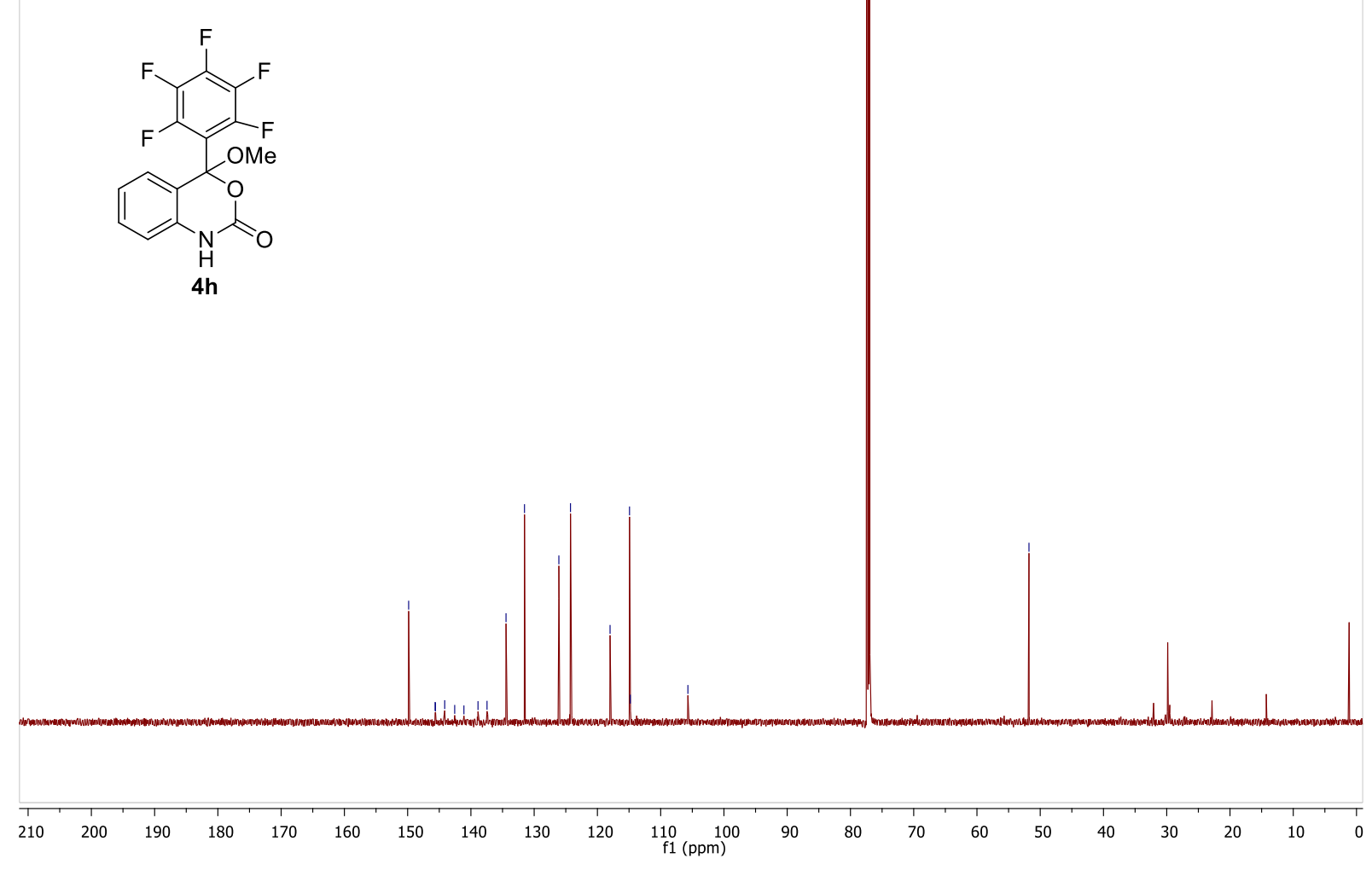

${ }^{13} \mathrm{C}\left\{{ }^{1} \mathrm{H}\right\} \mathrm{NMR}$ in $\mathrm{CDCl}_{3}$ at $176 \mathrm{MHz}$ 


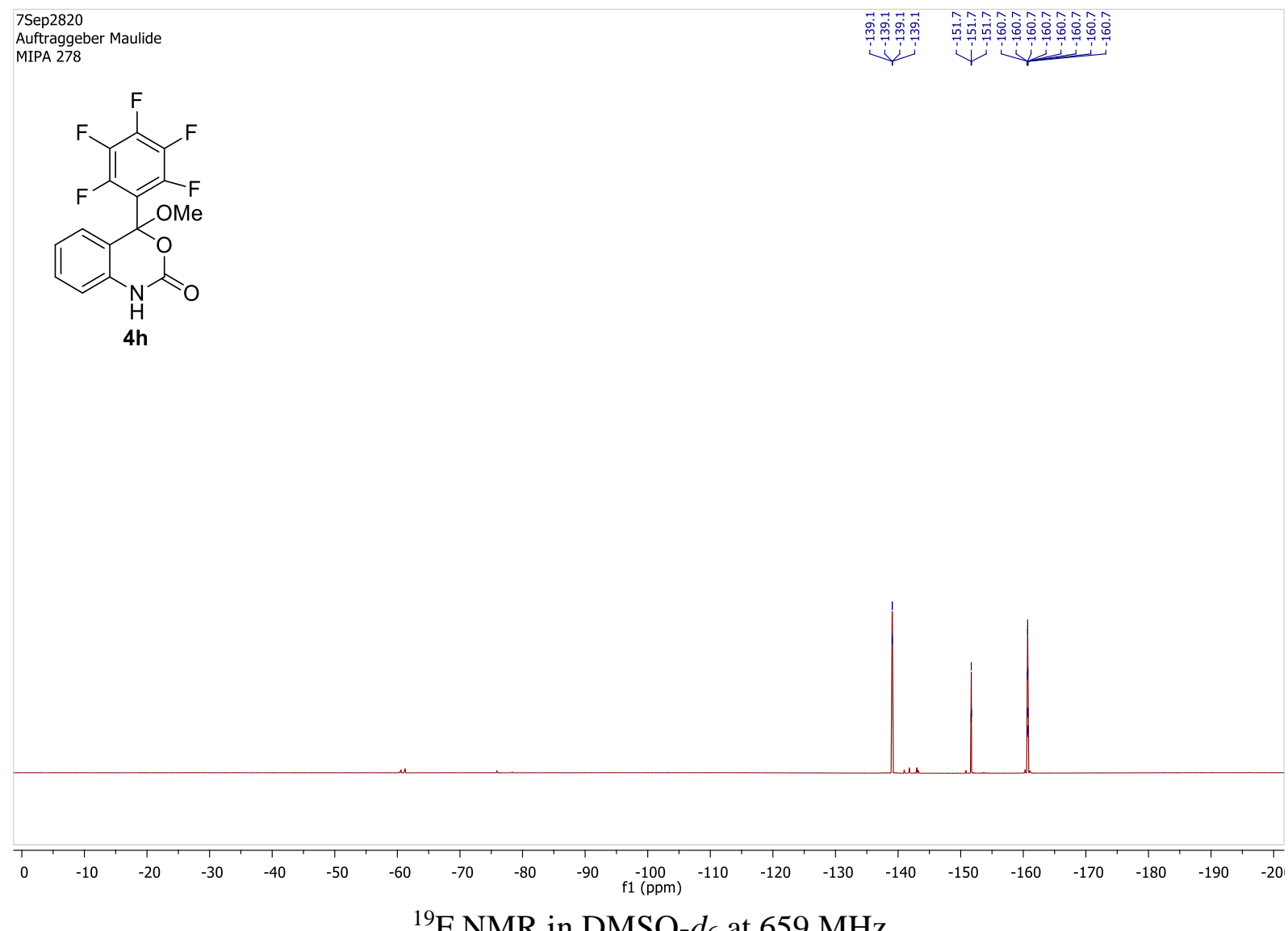

${ }^{19} \mathrm{~F}$ NMR in DMSO- $d_{6}$ at $659 \mathrm{MHz}$ 


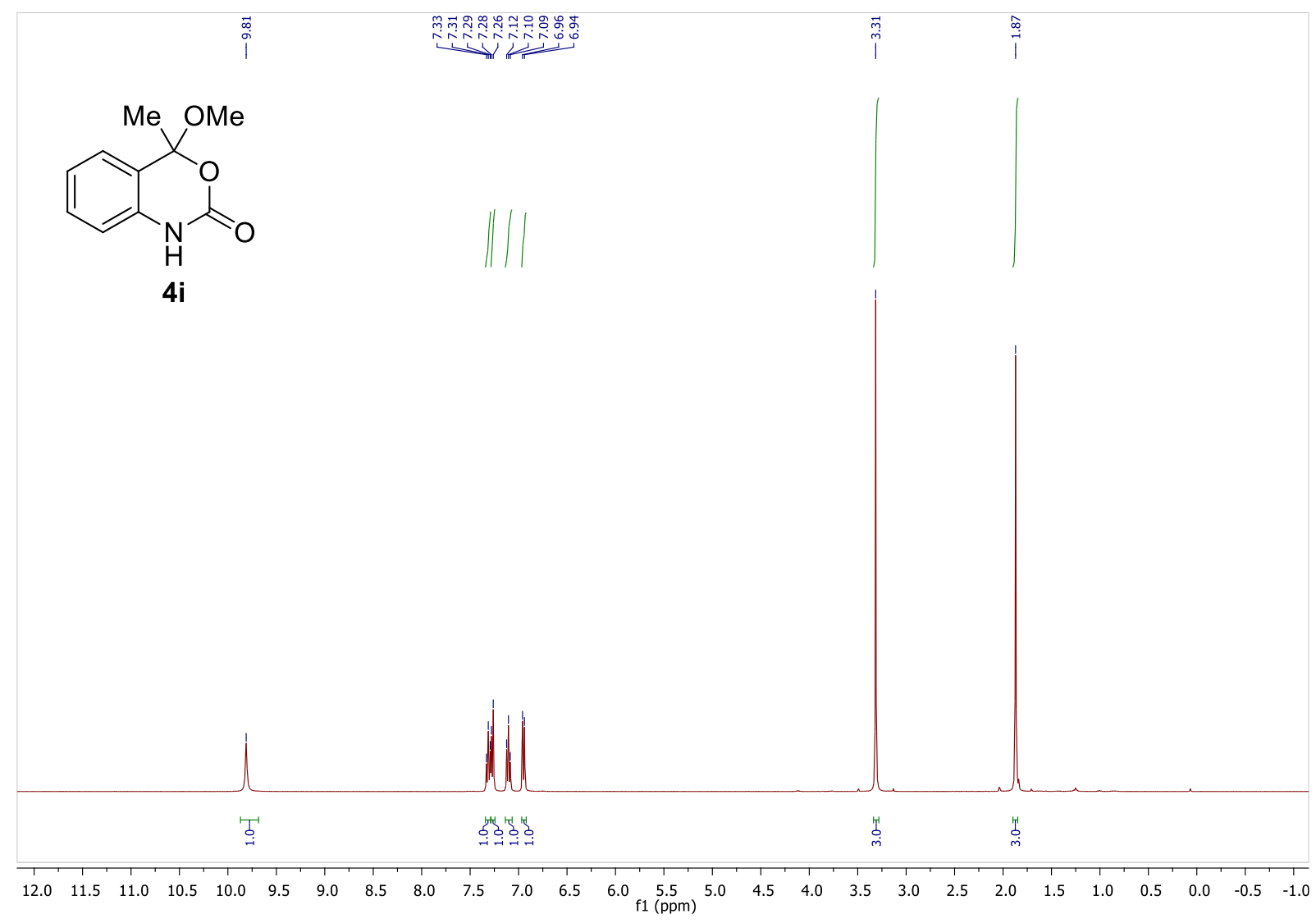

${ }^{1} \mathrm{H} \mathrm{NMR} \mathrm{in} \mathrm{CDCl}_{3}$ at $400 \mathrm{MHz}$

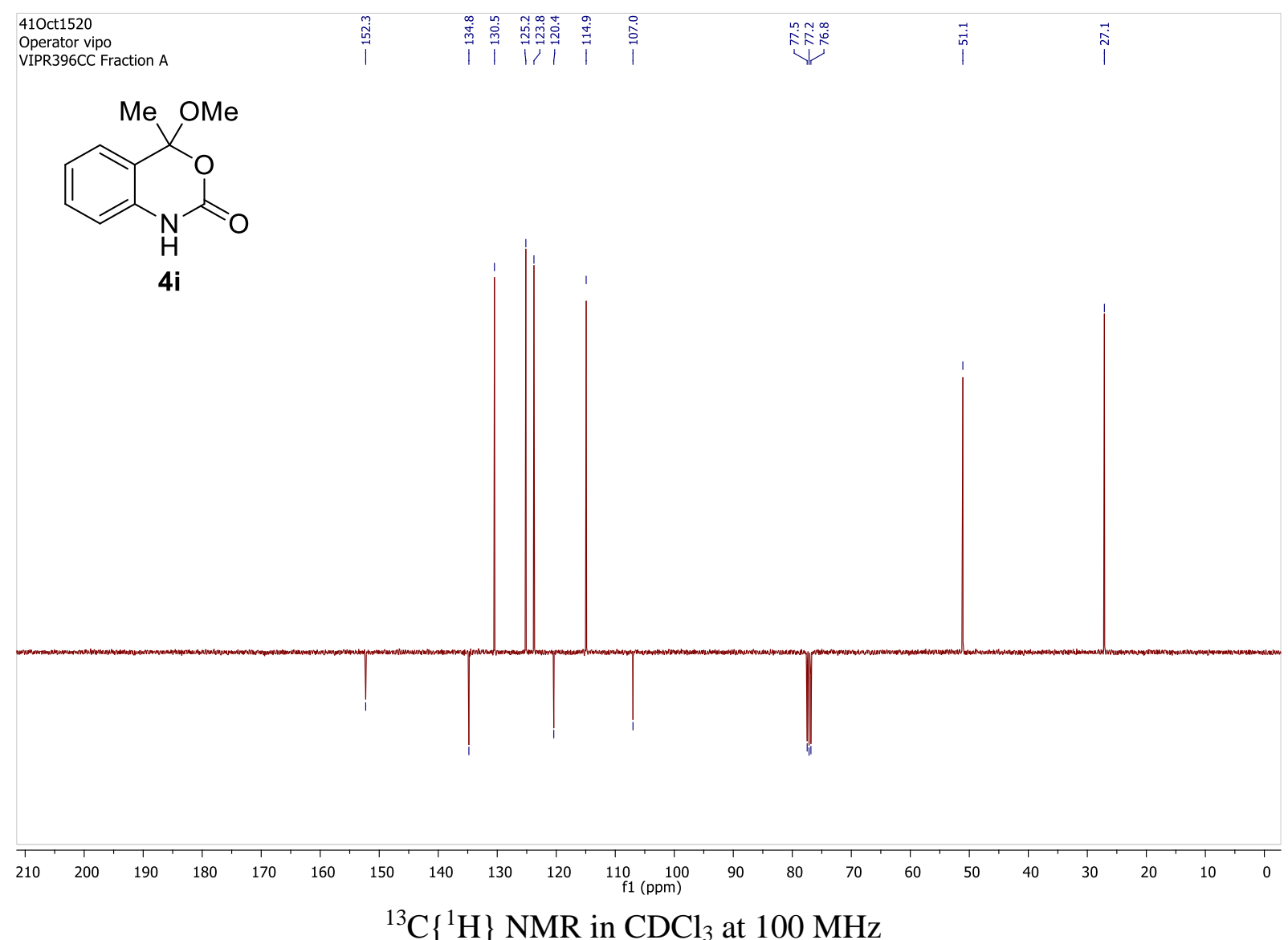

${ }^{13} \mathrm{C}\left\{{ }^{1} \mathrm{H}\right\} \mathrm{NMR}$ in $\mathrm{CDCl}_{3}$ at $100 \mathrm{MHz}$ 


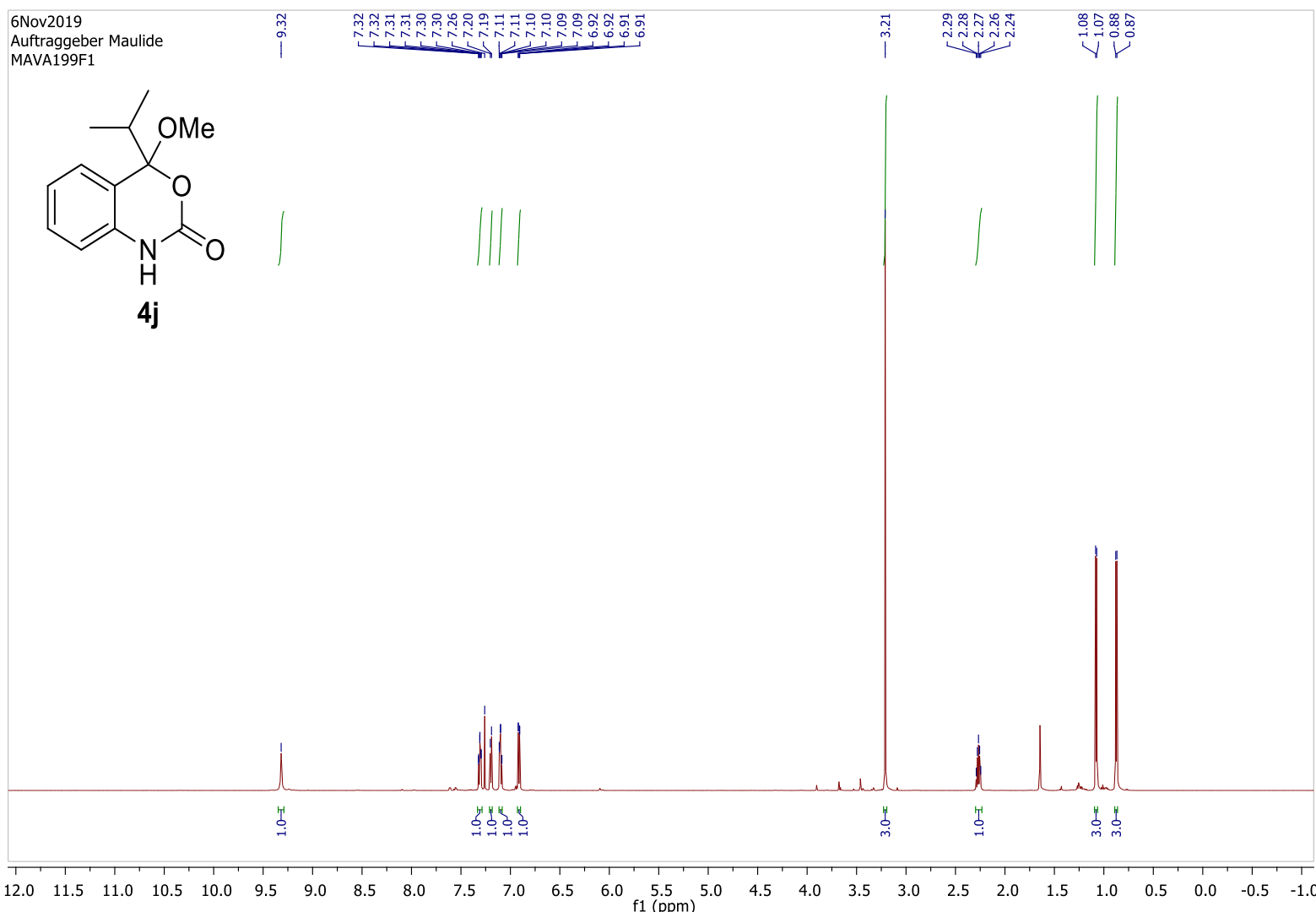

${ }^{1} \mathrm{H} \mathrm{NMR} \mathrm{in} \mathrm{CDCl}_{3}$ at $600 \mathrm{MHz}$

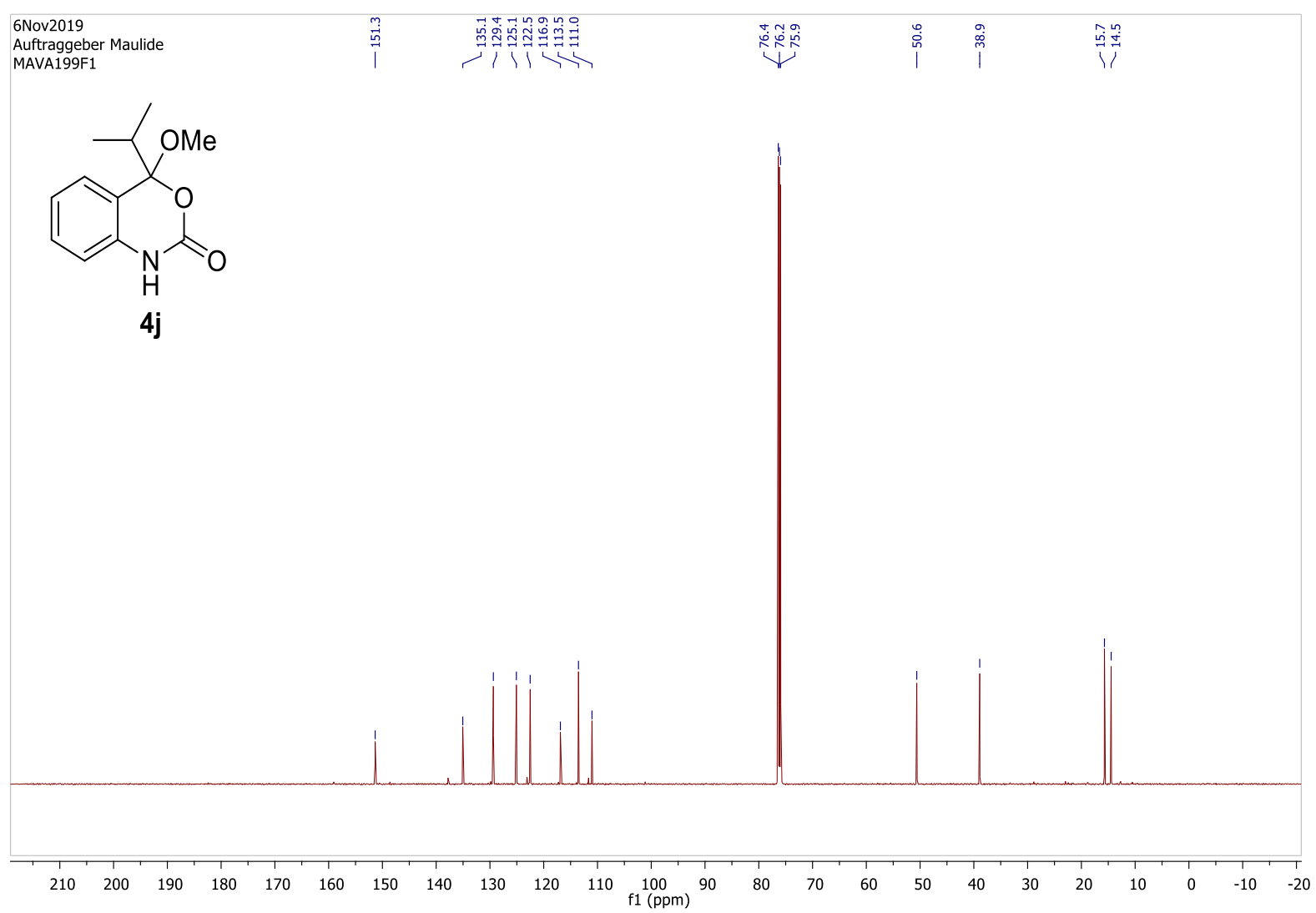

${ }^{13} \mathrm{C}\left\{{ }^{1} \mathrm{H}\right\} \mathrm{NMR}$ in $\mathrm{CDCl}_{3}$ at $150 \mathrm{MHz}$ 


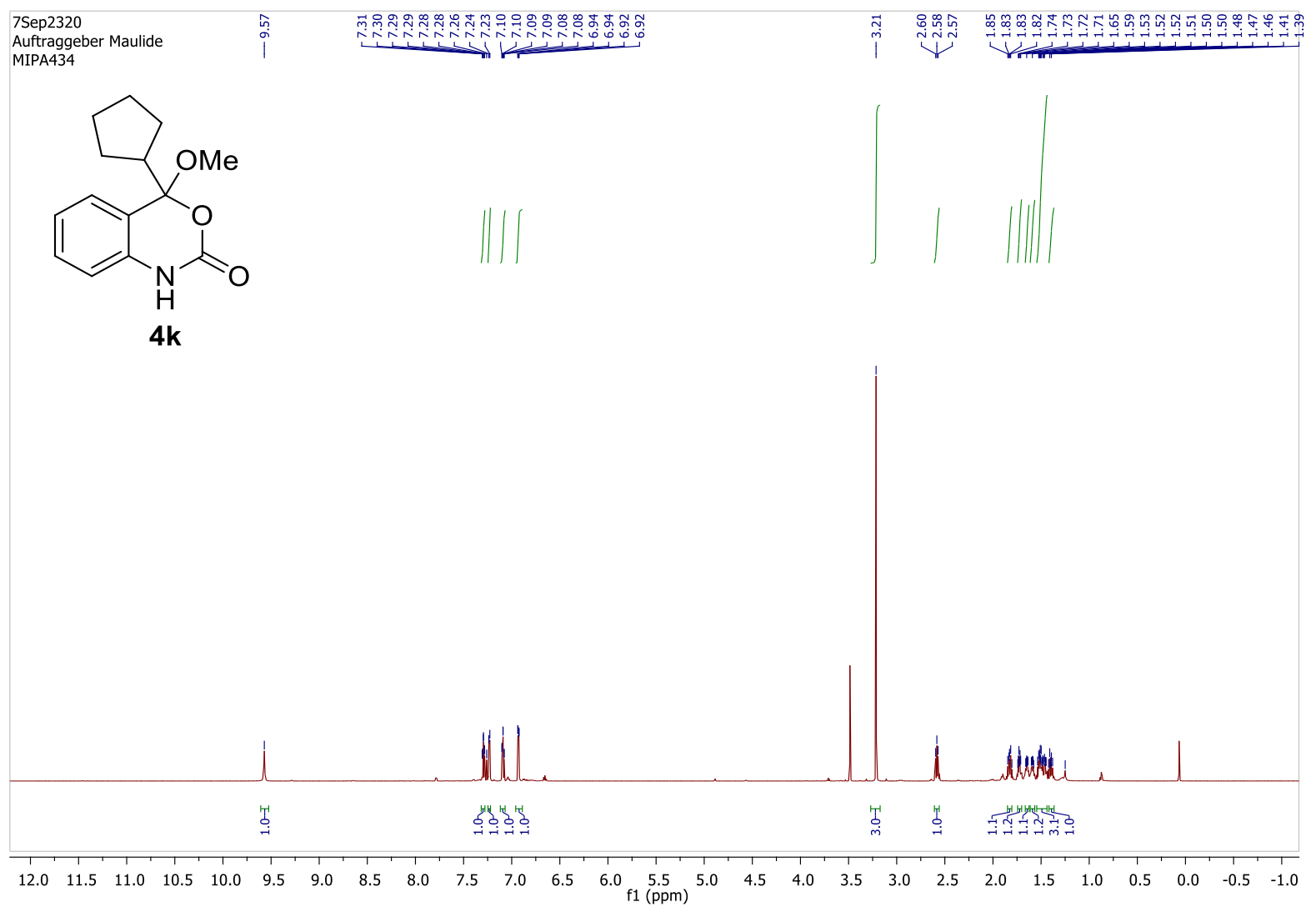

${ }^{1} \mathrm{H} \mathrm{NMR}$ in $\mathrm{CDCl}_{3}$ at $700 \mathrm{MHz}$

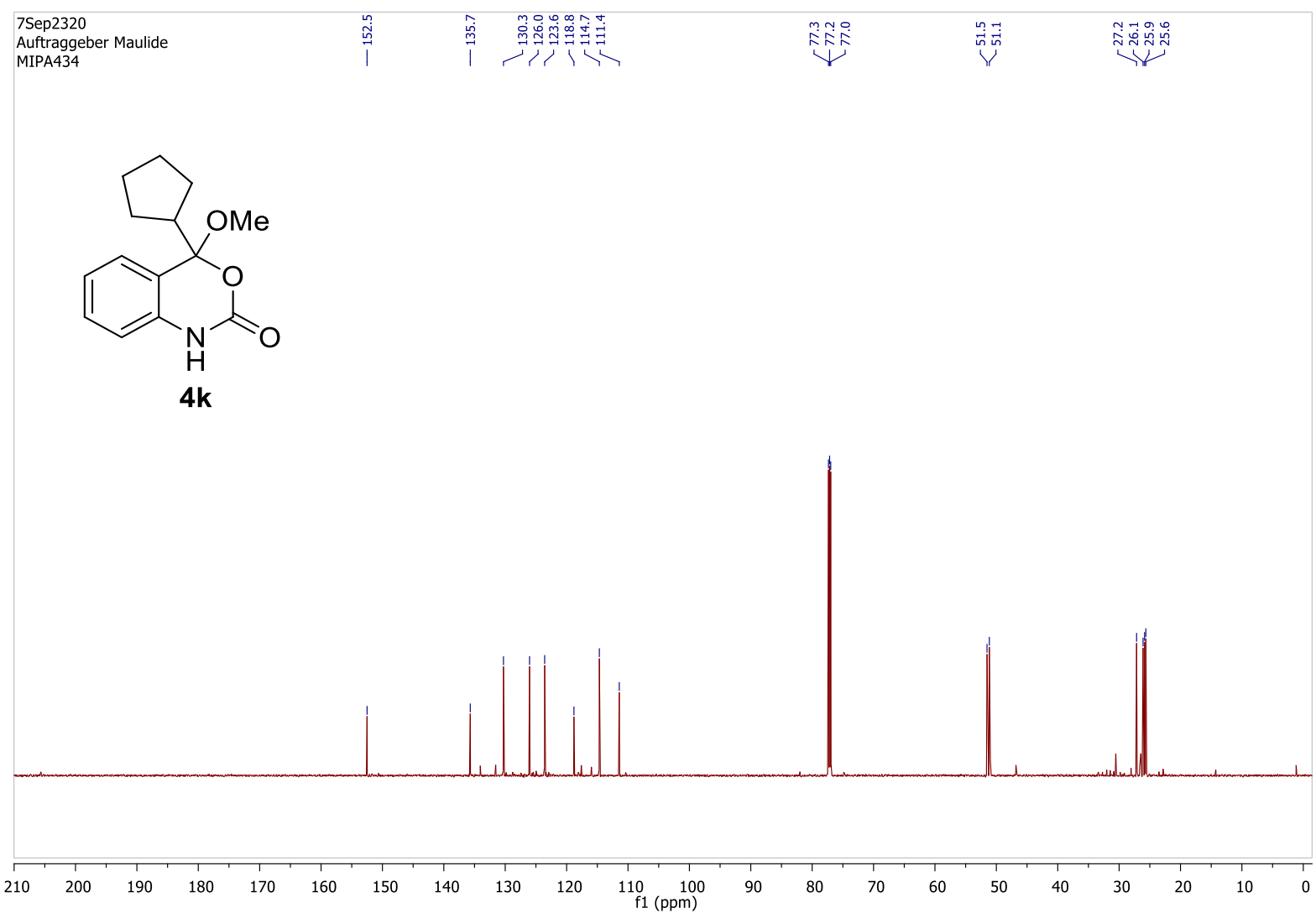

${ }^{13} \mathrm{C}\left\{{ }^{1} \mathrm{H}\right\} \mathrm{NMR}$ in $\mathrm{CDCl}_{3}$ at $176 \mathrm{MHz}$ 


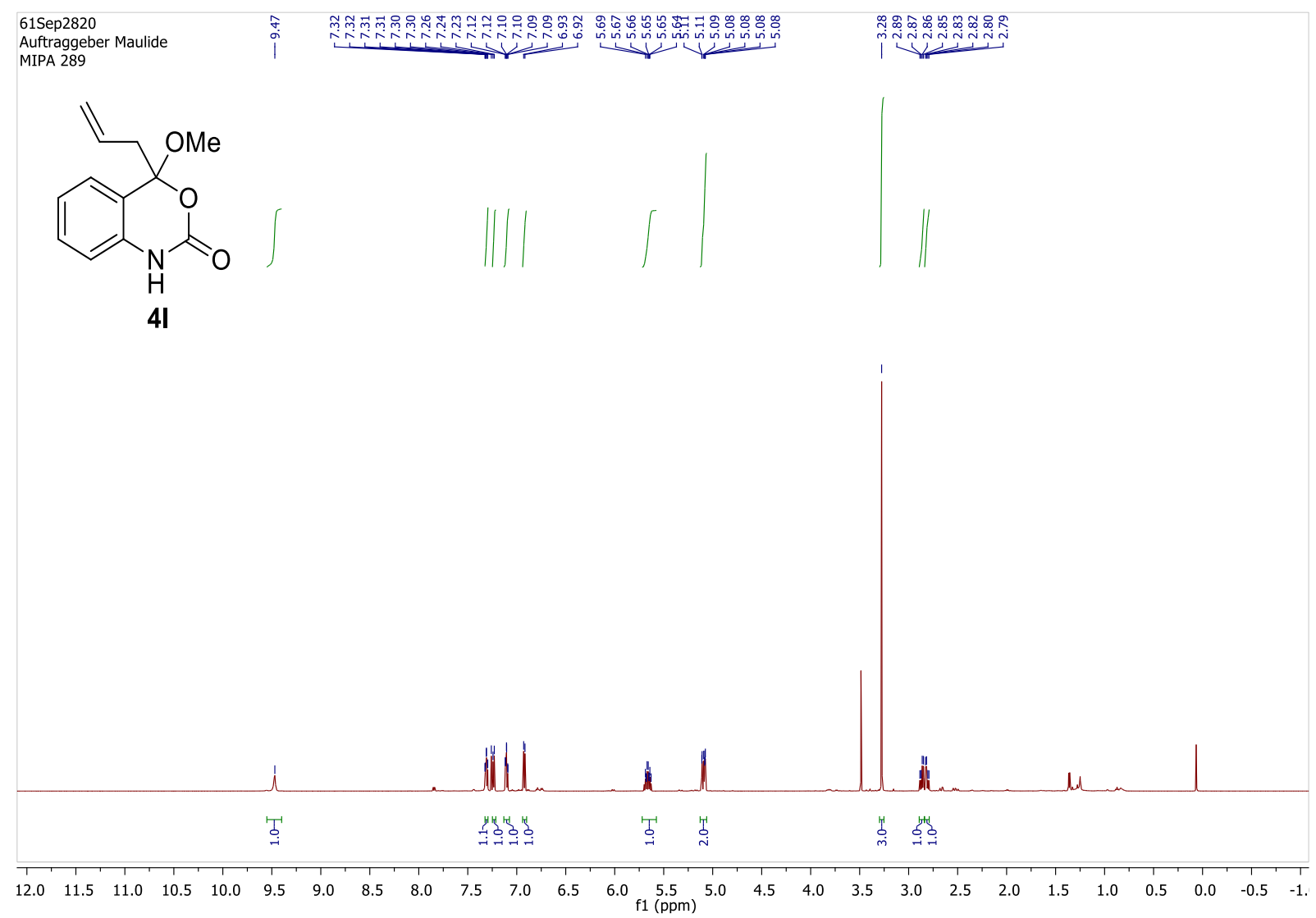

${ }^{1} \mathrm{H} \mathrm{NMR}$ in $\mathrm{CDCl}_{3}$ at $700 \mathrm{MHz}$

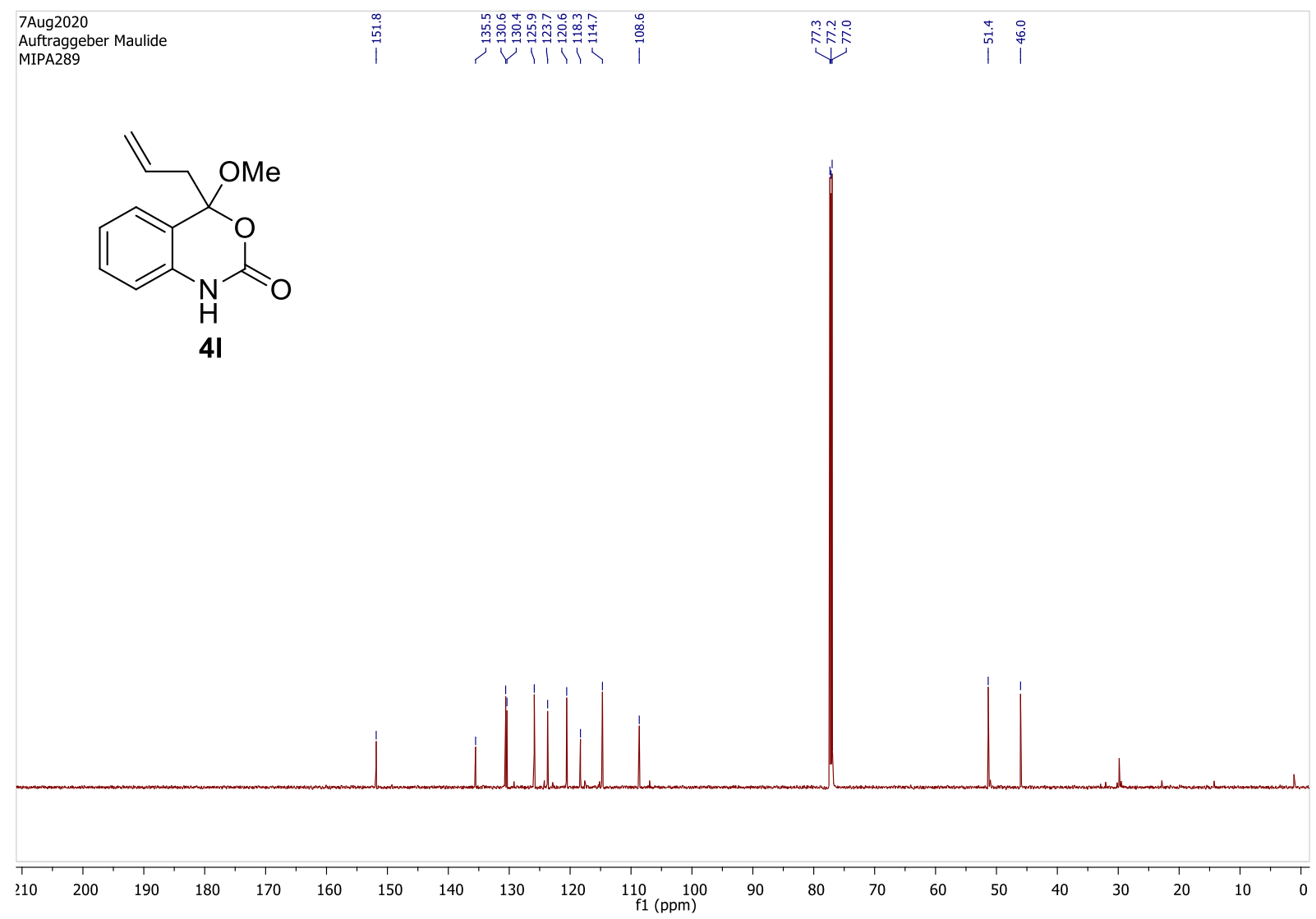

${ }^{13} \mathrm{C}\left\{{ }^{1} \mathrm{H}\right\} \mathrm{NMR}$ in $\mathrm{CDCl}_{3}$ at $176 \mathrm{MHz}$ 


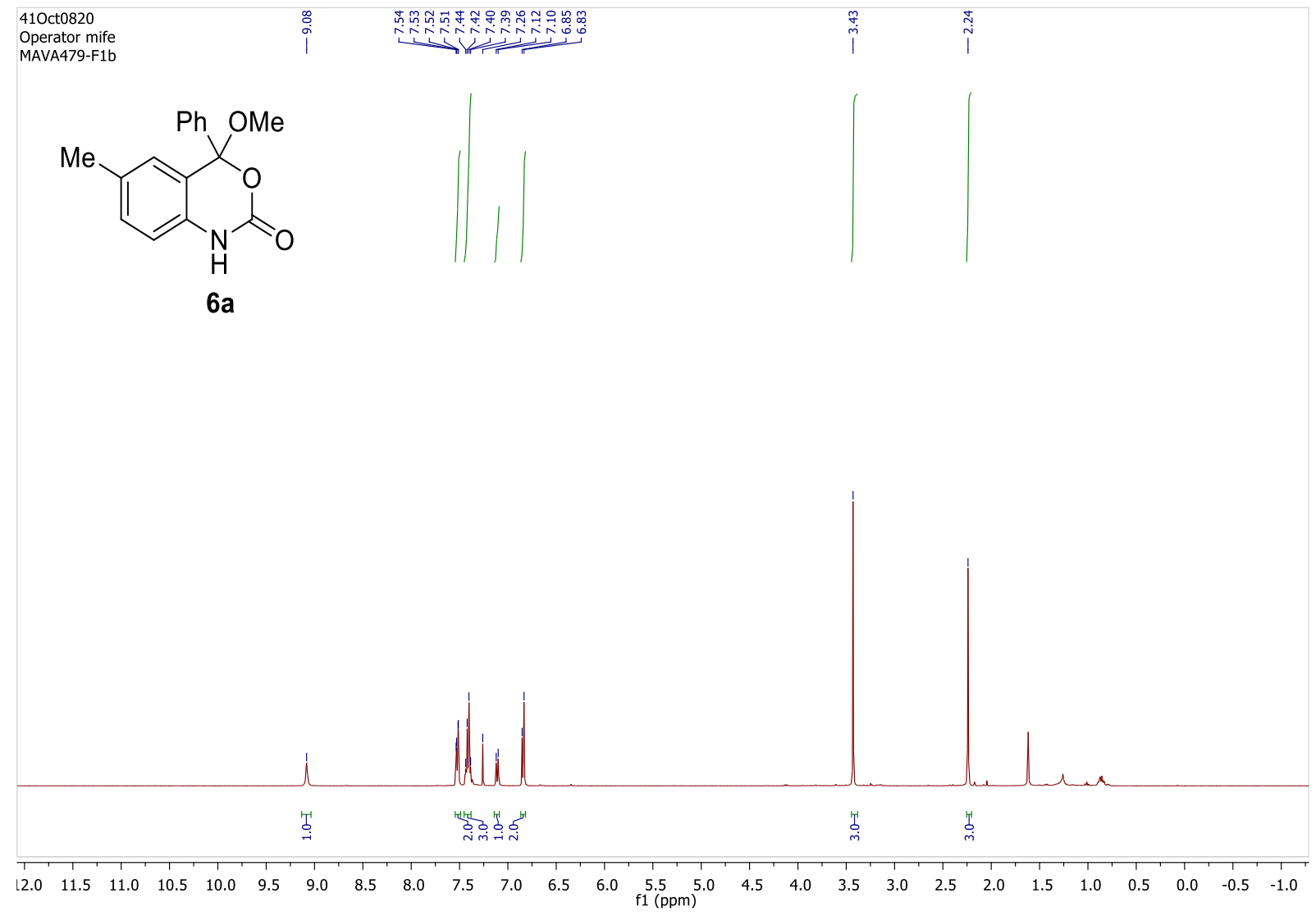

${ }^{1} \mathrm{H} \mathrm{NMR}$ in $\mathrm{CDCl}_{3}$ at $400 \mathrm{MHz}$

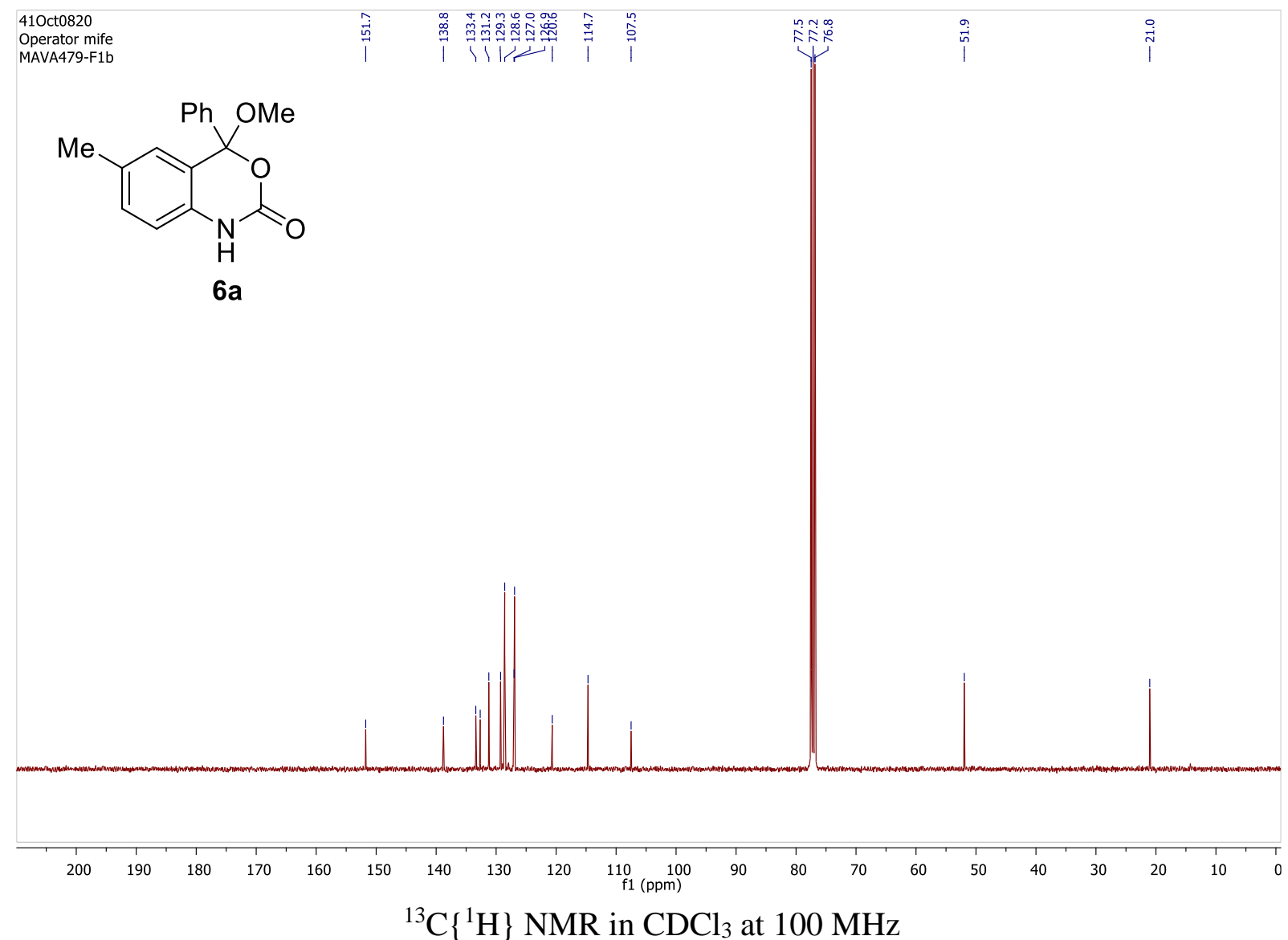




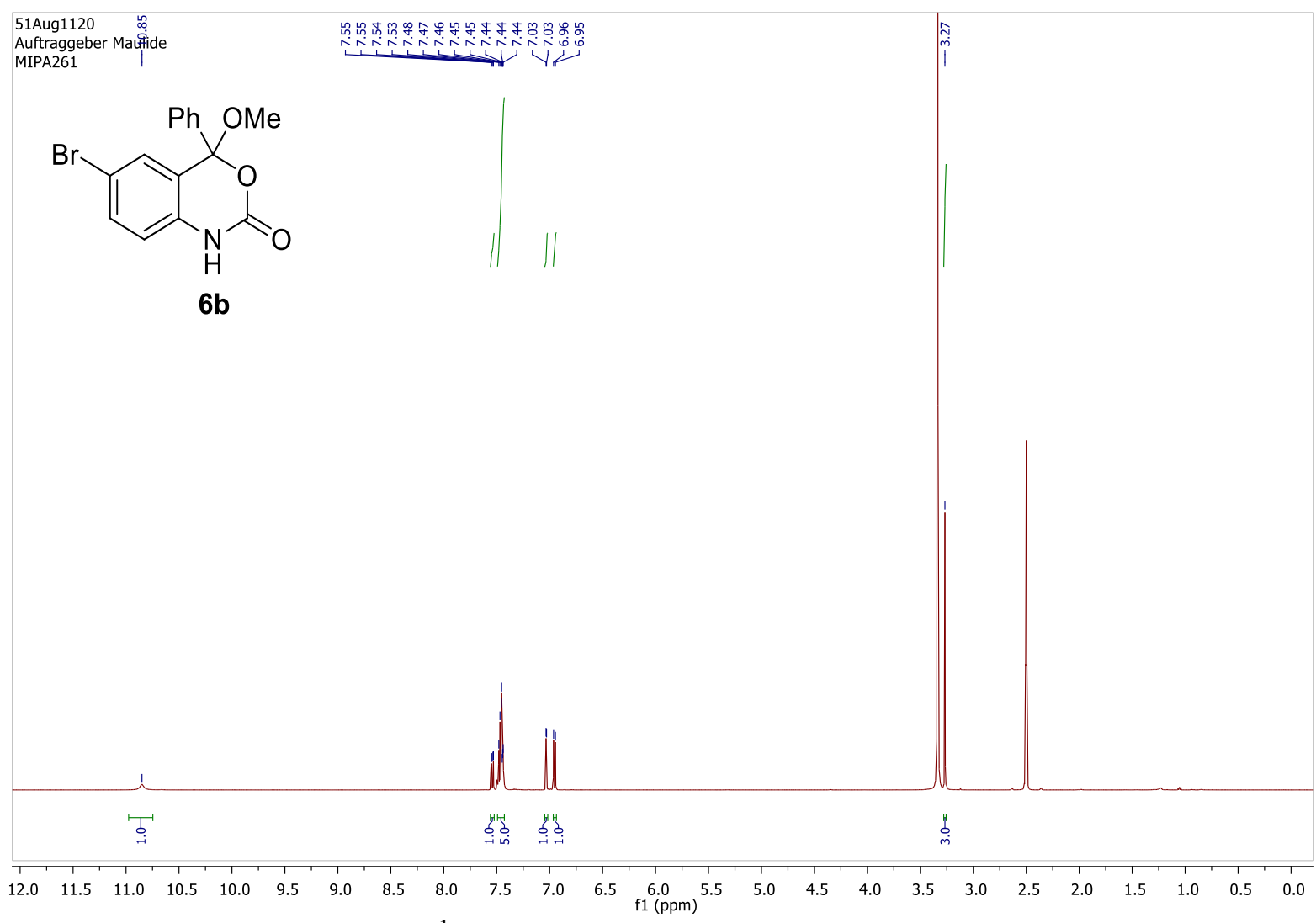

${ }^{1} \mathrm{H} \mathrm{NMR}$ in $\mathrm{CDCl}_{3}$ at $500 \mathrm{MHz}$

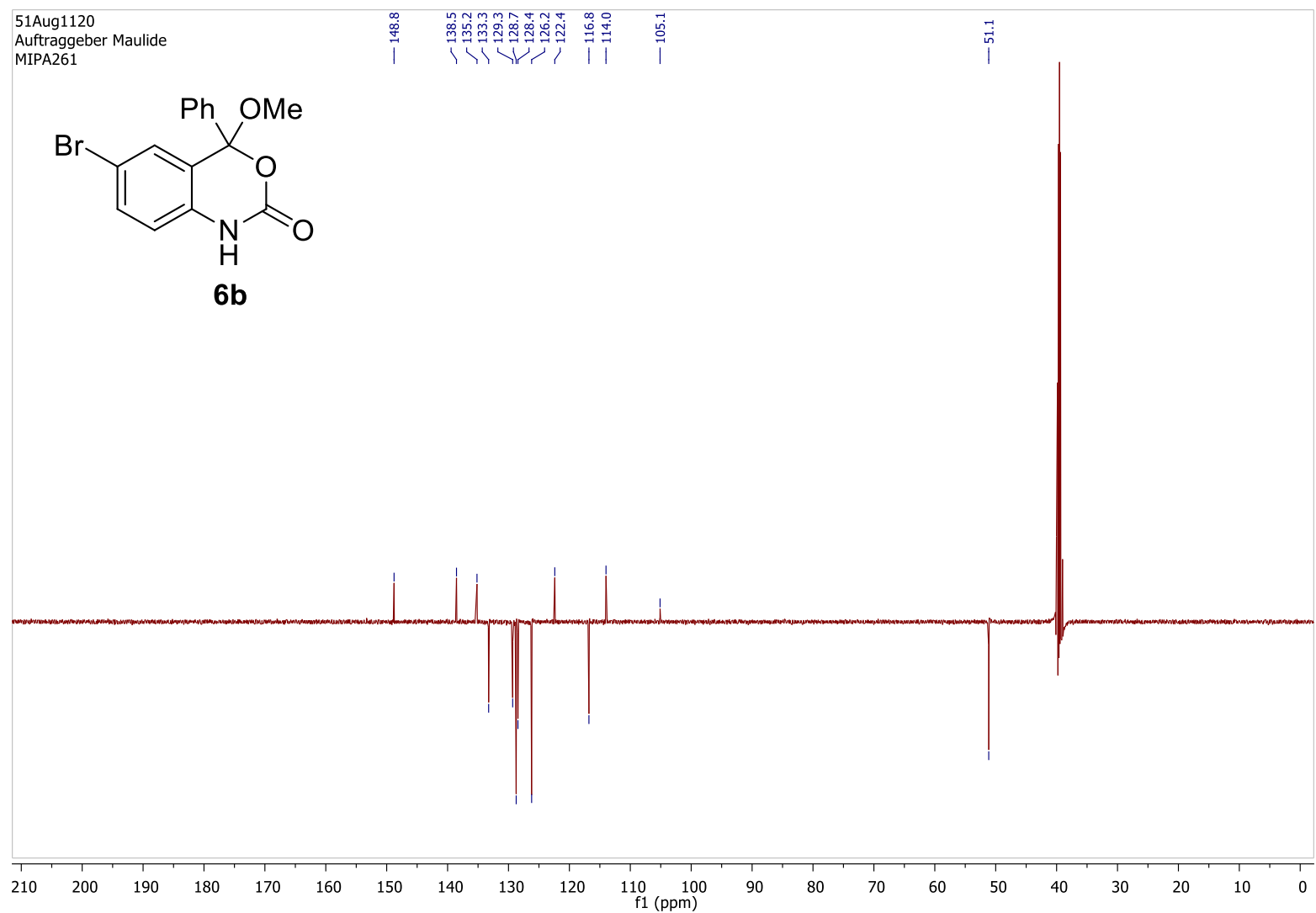

${ }^{13} \mathrm{C}\left\{{ }^{1} \mathrm{H}\right\} \mathrm{NMR}$ in $\mathrm{CDCl}_{3}$ at $126 \mathrm{MHz}$ 


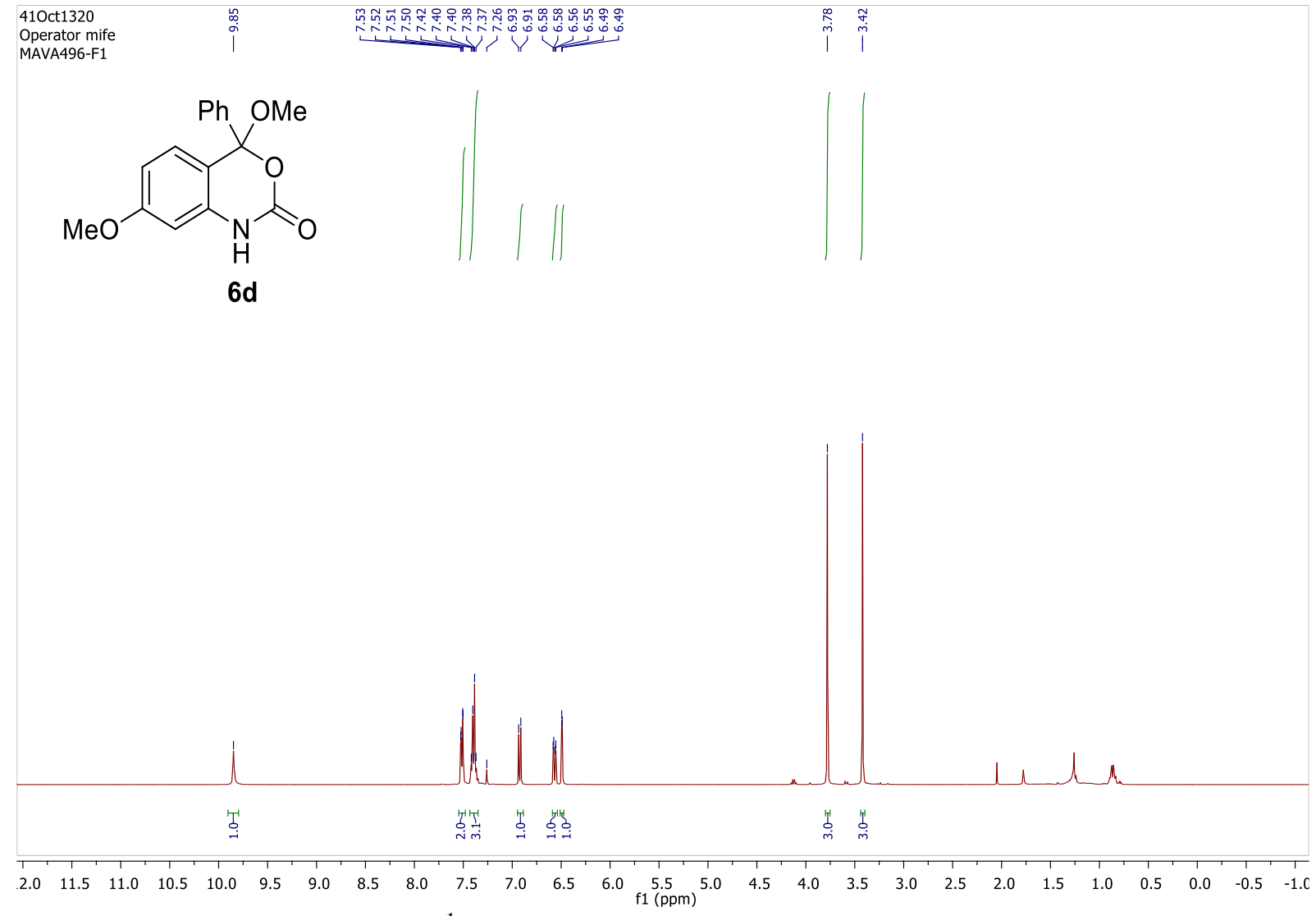

${ }^{1} \mathrm{H} \mathrm{NMR}$ in $\mathrm{CDCl}_{3}$ at $400 \mathrm{MHz}$

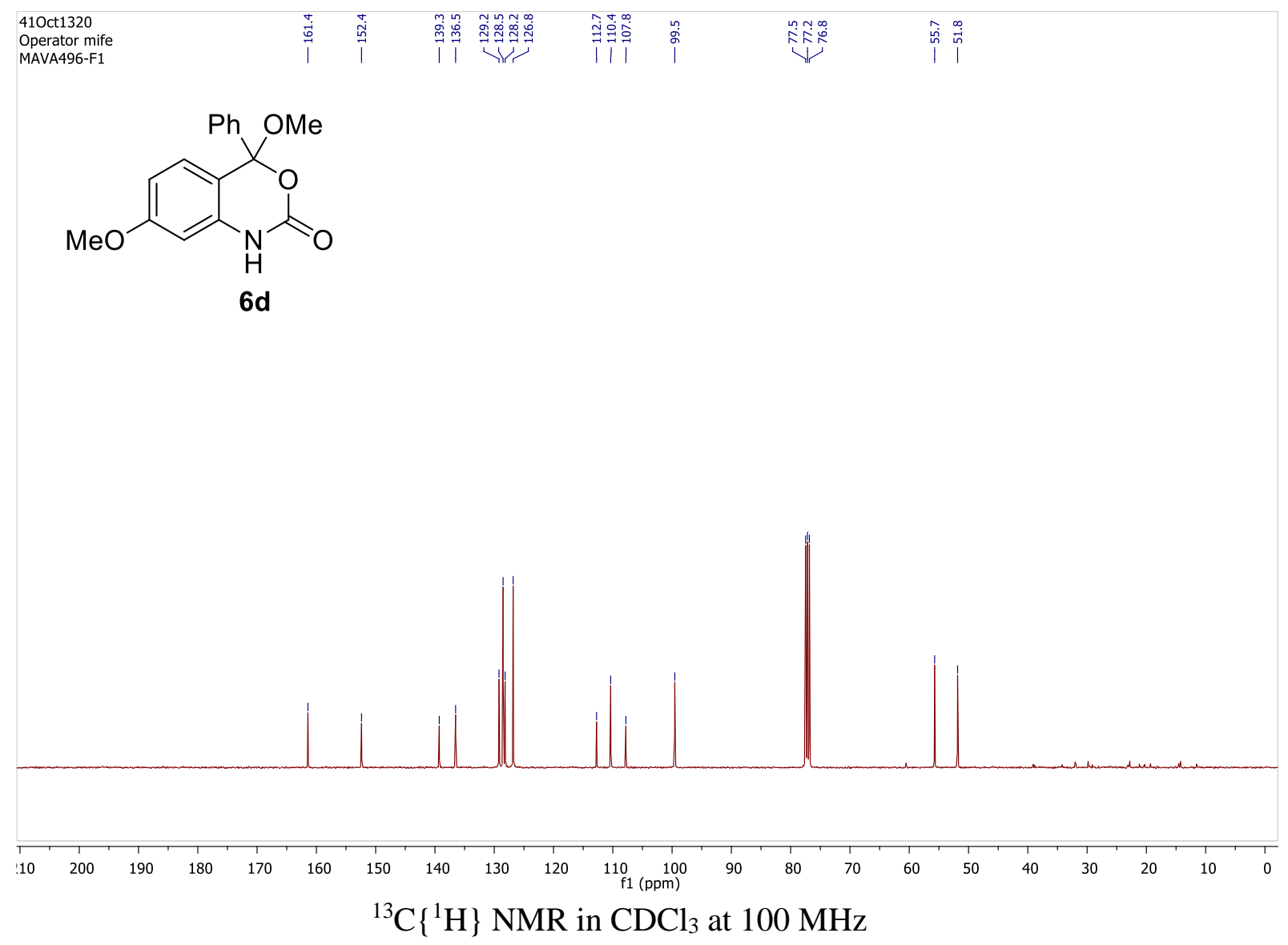


<smiles>COC1(c2ccccc2)OC(=O)Nc2c(C)cccc21</smiles><smiles>Cc1ccccc1</smiles>

$6 e$

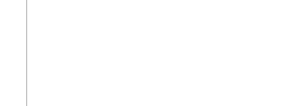<smiles>CCC</smiles>

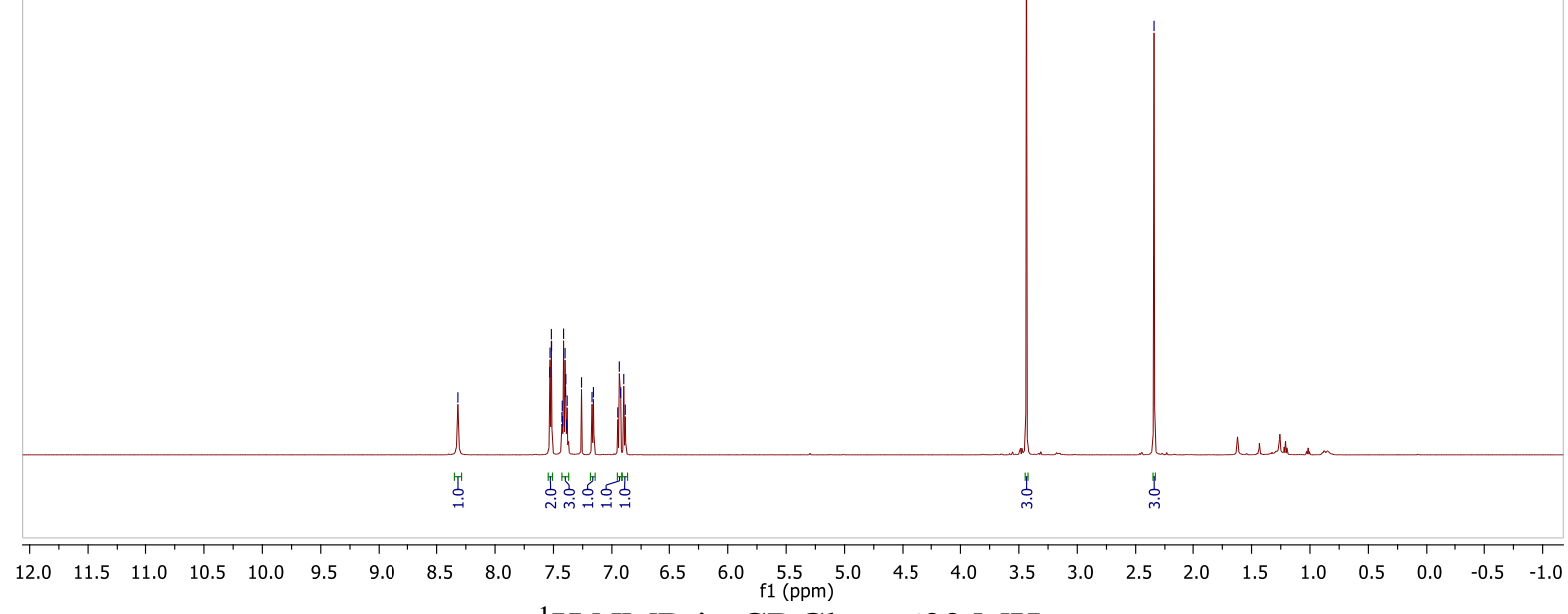

${ }^{1} \mathrm{H} \mathrm{NMR}$ in $\mathrm{CDCl}_{3}$ at $600 \mathrm{MHz}$

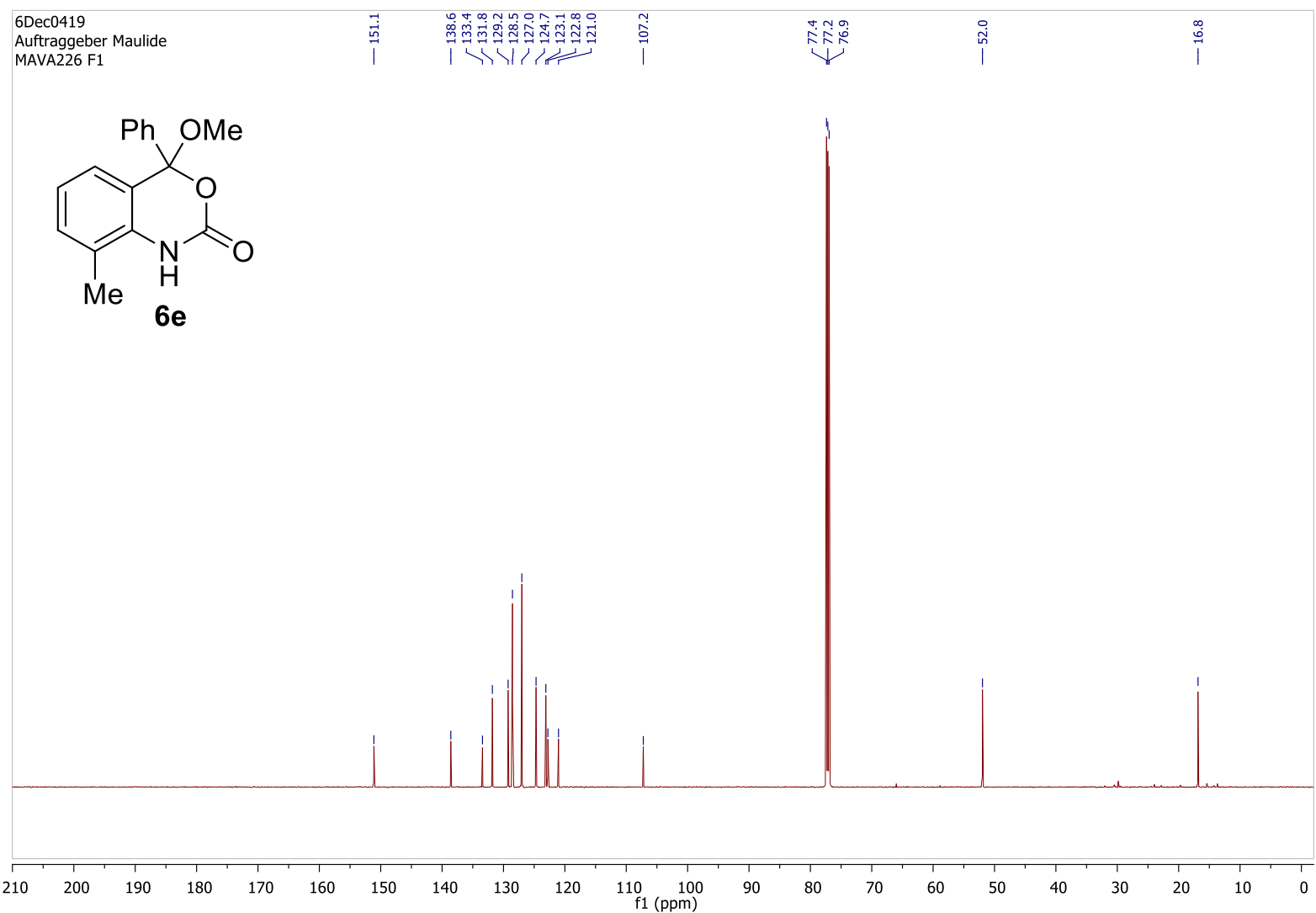

${ }^{13} \mathrm{C}\left\{{ }^{1} \mathrm{H}\right\} \mathrm{NMR}$ in $\mathrm{CDCl}_{3}$ at $150 \mathrm{MHz}$ 


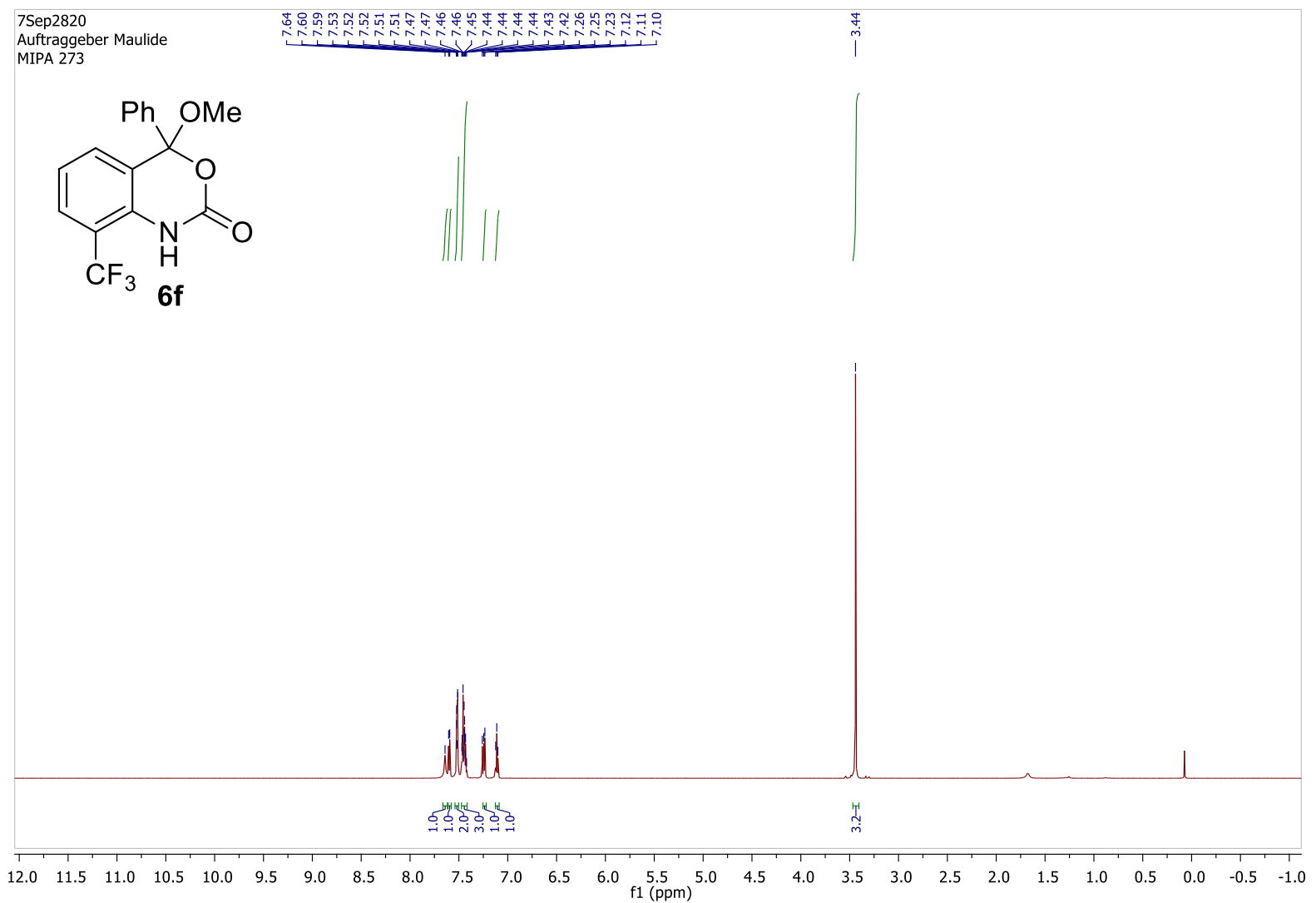

${ }^{1} \mathrm{H} \mathrm{NMR}$ in $\mathrm{CDCl}_{3}$ at $700 \mathrm{MHz}$

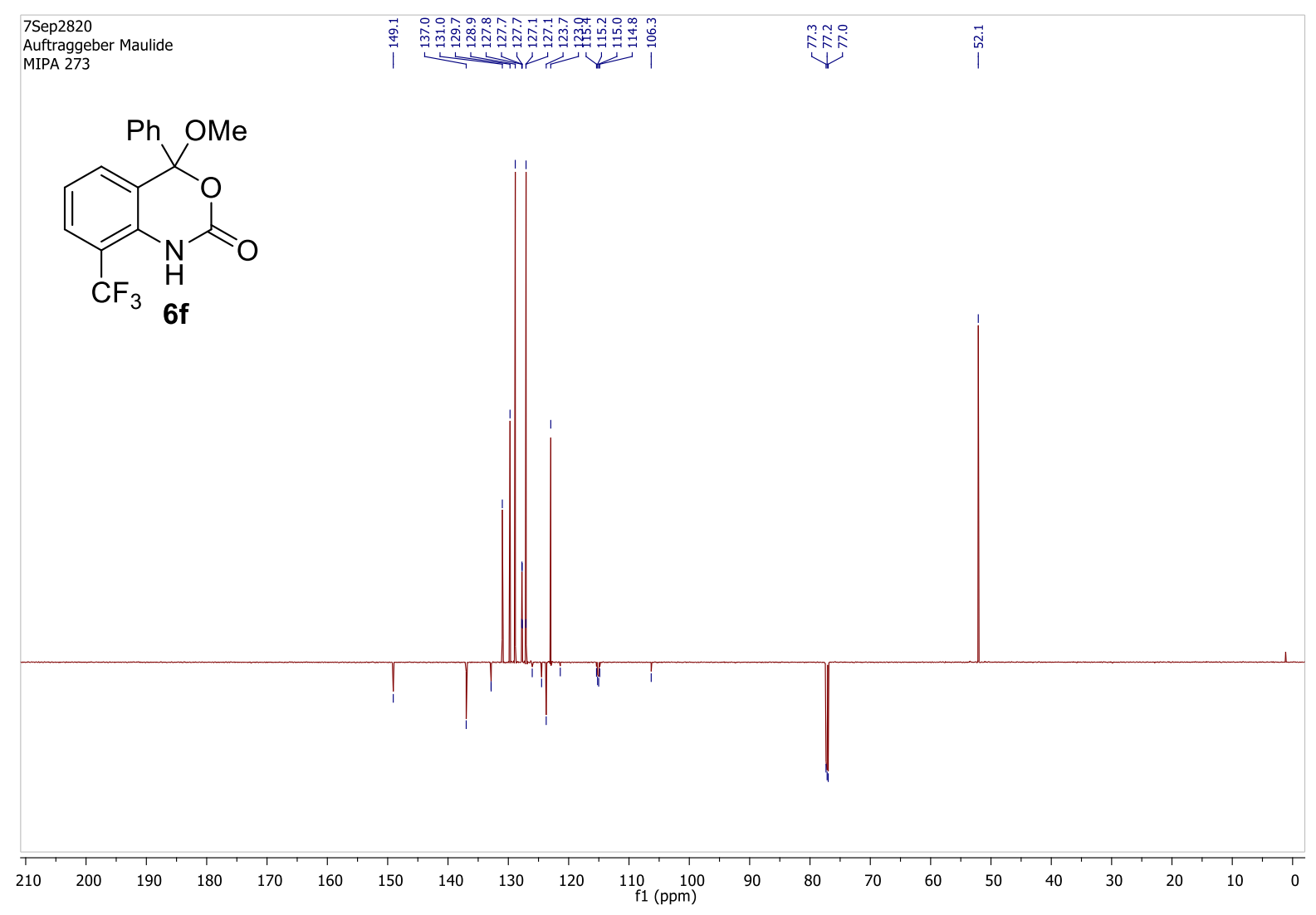

${ }^{13} \mathrm{C}\left\{{ }^{1} \mathrm{H}\right\} \mathrm{NMR}$ in $\mathrm{CDCl}_{3}$ at $175 \mathrm{MHz}$ 
<smiles>COC1(c2ccccc2)OC(=O)Nc2c(C(F)(F)F)cccc21</smiles>

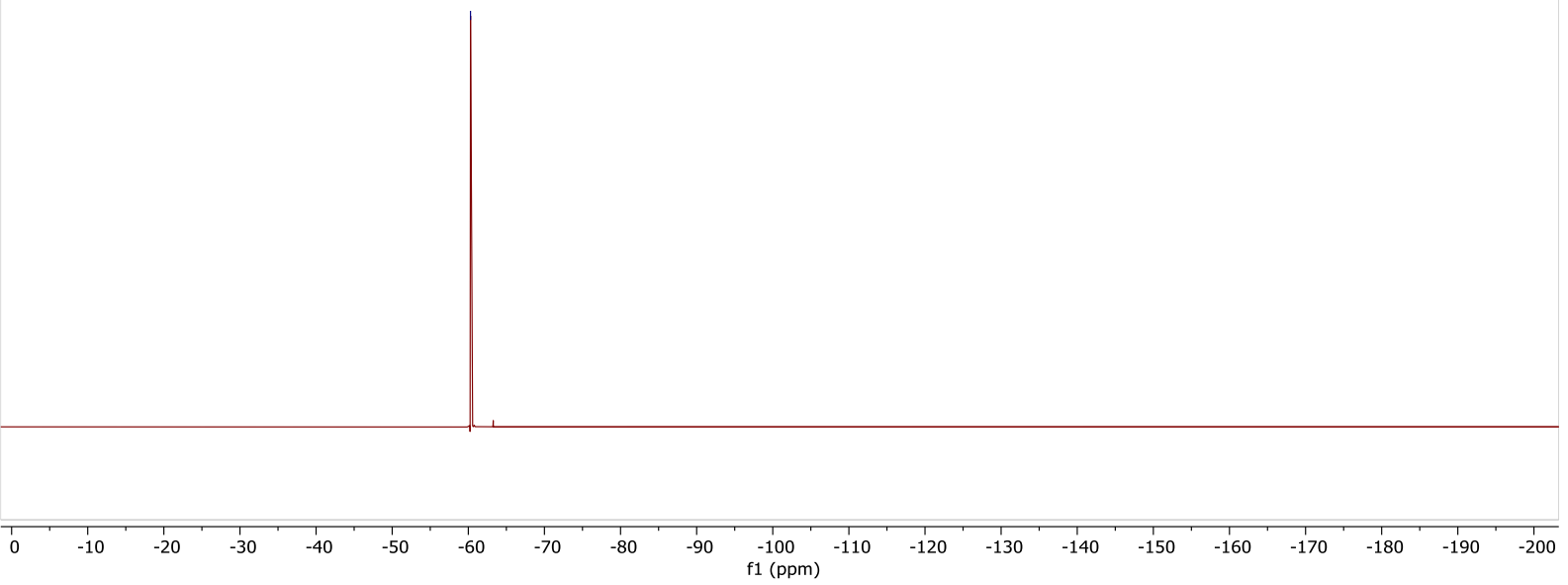

${ }^{19} \mathrm{~F}$ NMR in DMSO- $d_{6}$ at $376 \mathrm{MHz}$ 


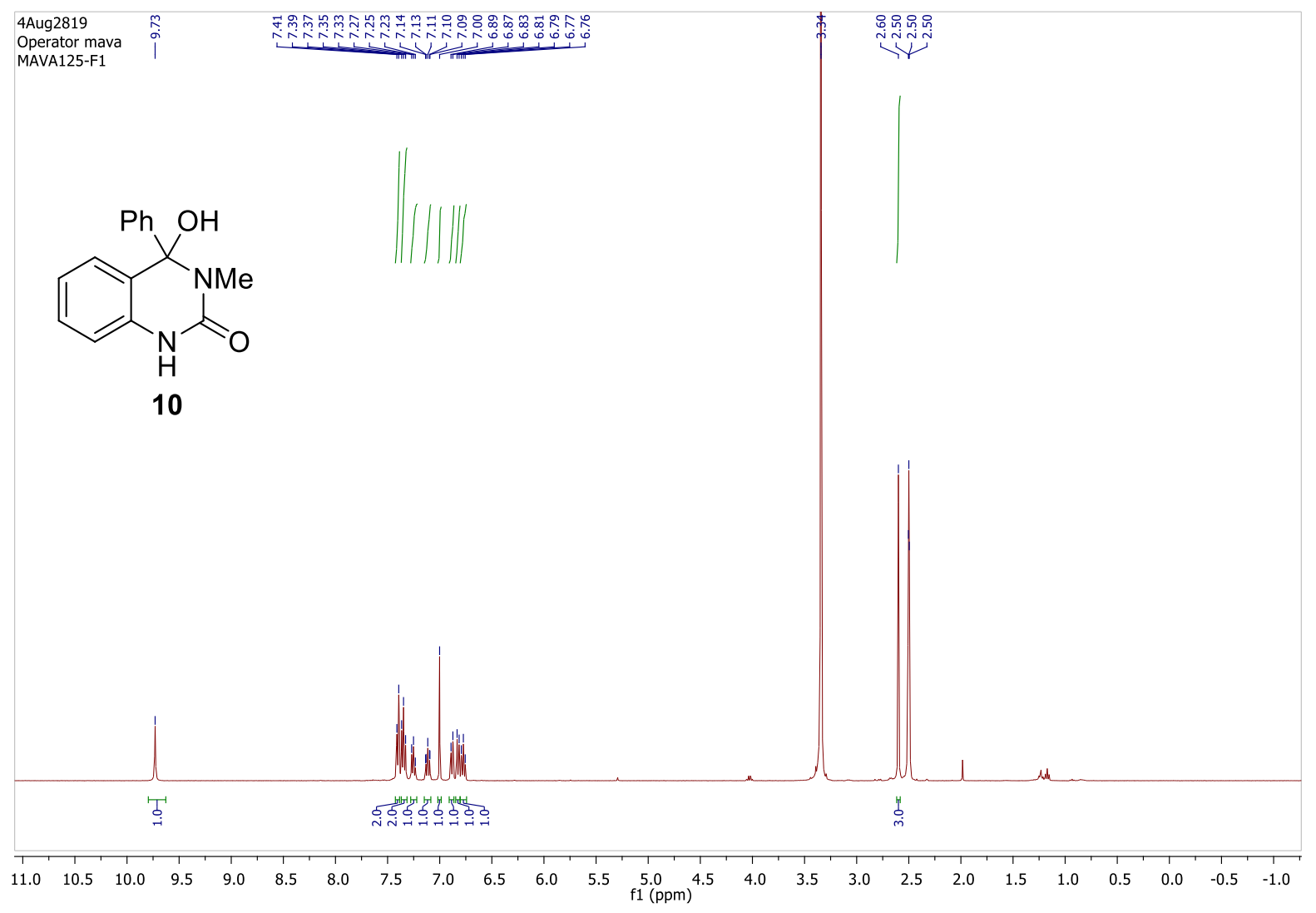

${ }^{1} \mathrm{H}$ NMR in $\mathrm{CDCl}_{3}$ at $400 \mathrm{MHz}$

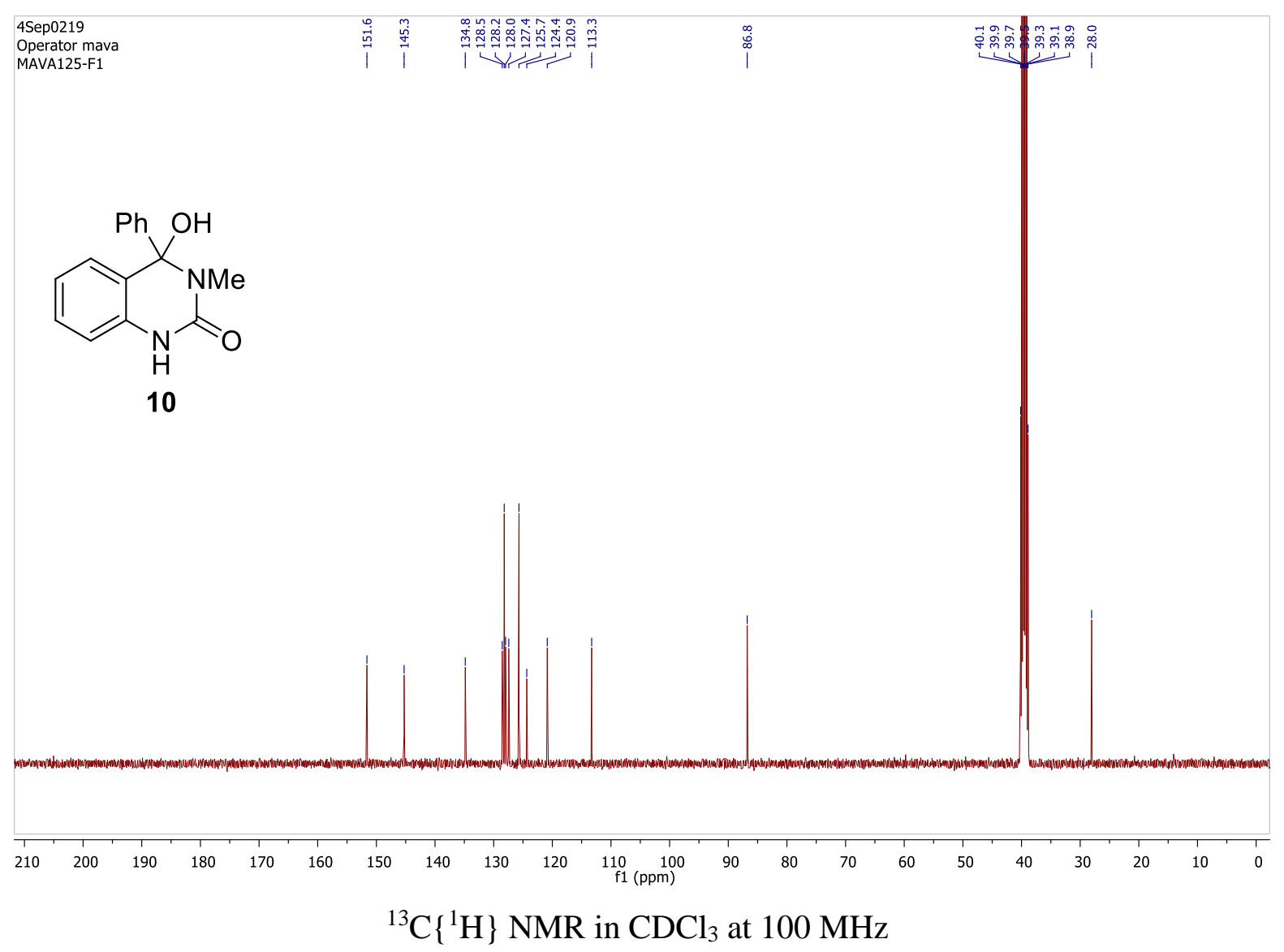




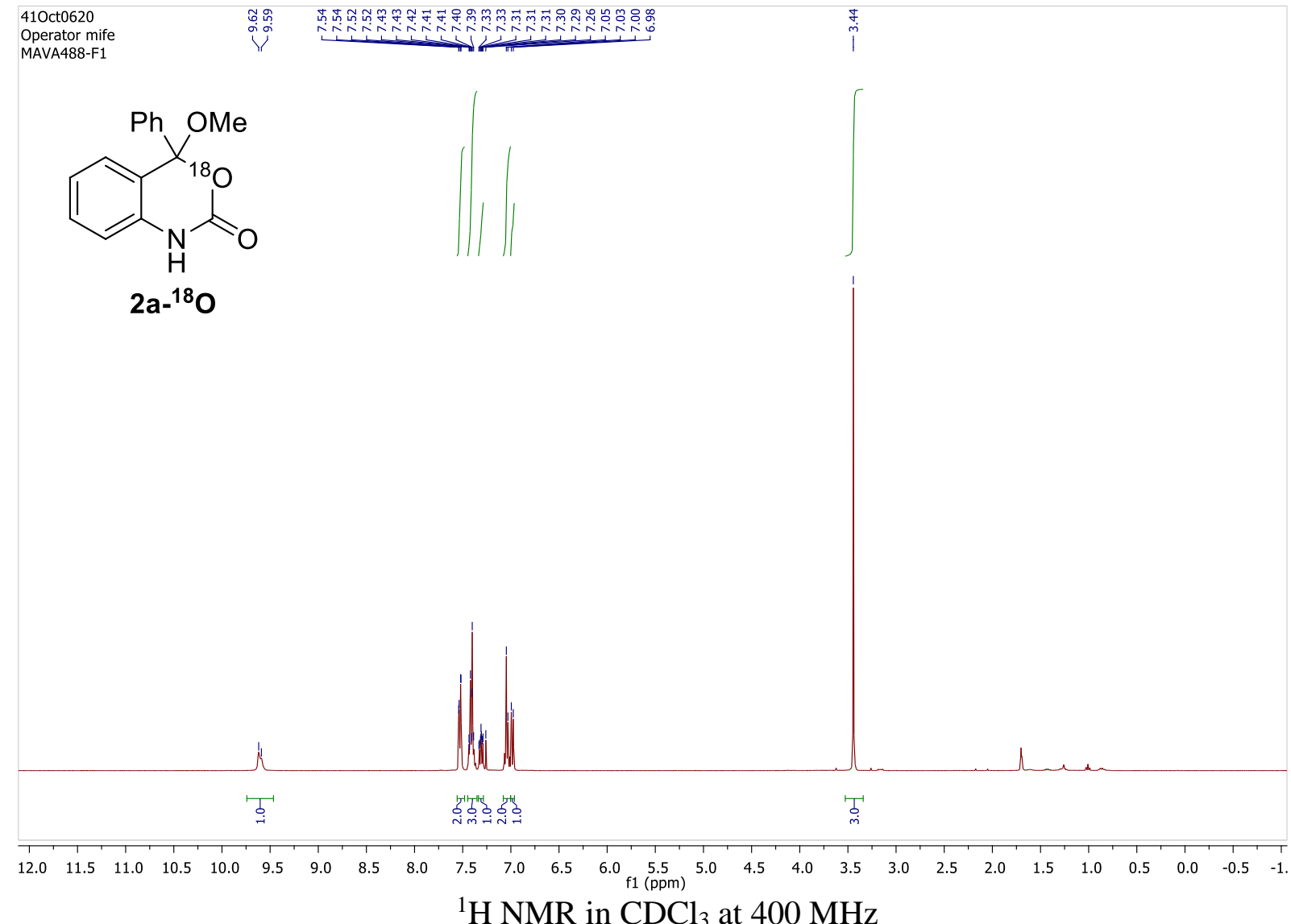

${ }^{1} \mathrm{H}$ NMR in $\mathrm{CDCl}_{3}$ at $400 \mathrm{MHz}$

$410 c t 0620$

Operator mife

|<smiles>CO[C@@]1(c2ccccc2)OC(=O)Nc2ccccc21</smiles>

$2 a-{ }^{18} \mathrm{O}$

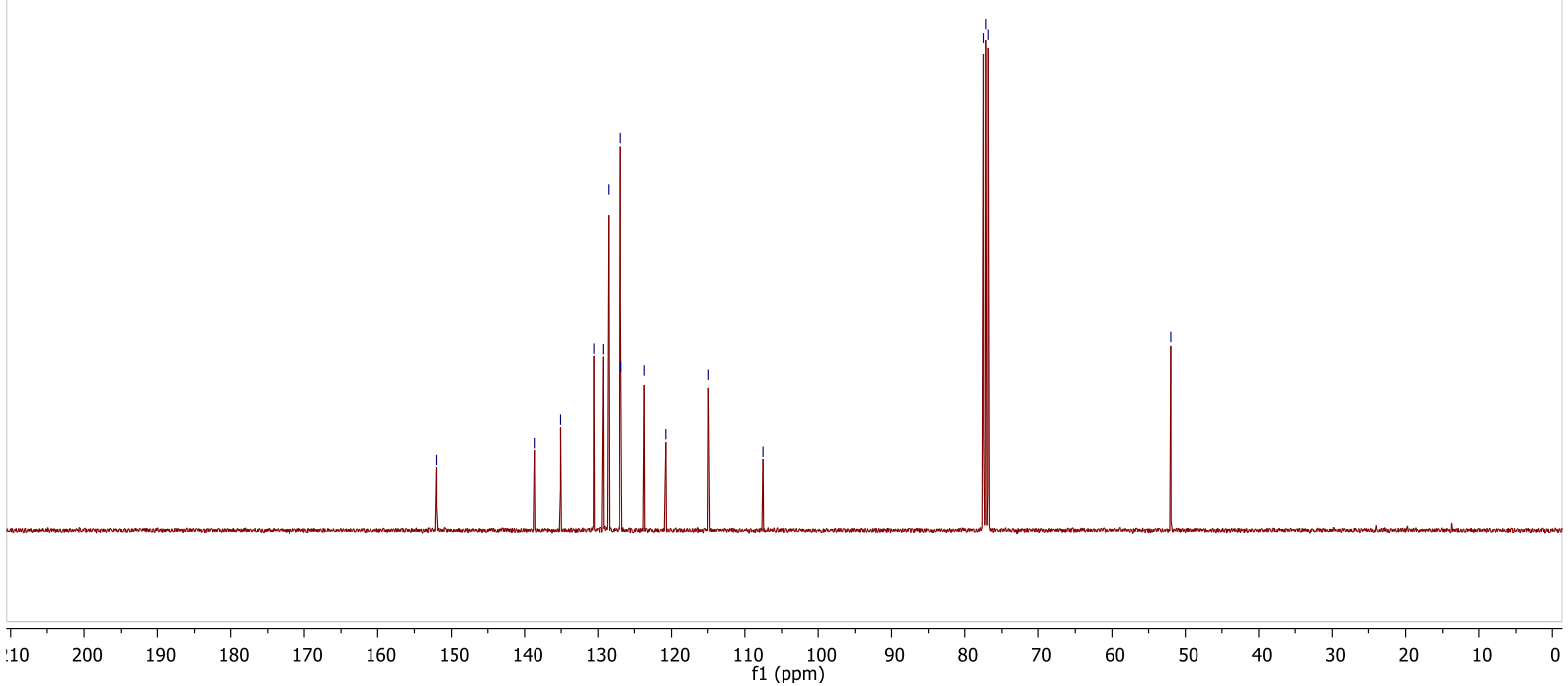

${ }^{13} \mathrm{C}\left\{{ }^{1} \mathrm{H}\right\} \mathrm{NMR}$ in $\mathrm{CDCl}_{3}$ at $100 \mathrm{MHz}$ 
<smiles>COC(=O)Nc1ccccc1C(=O)c1ccccc1</smiles>
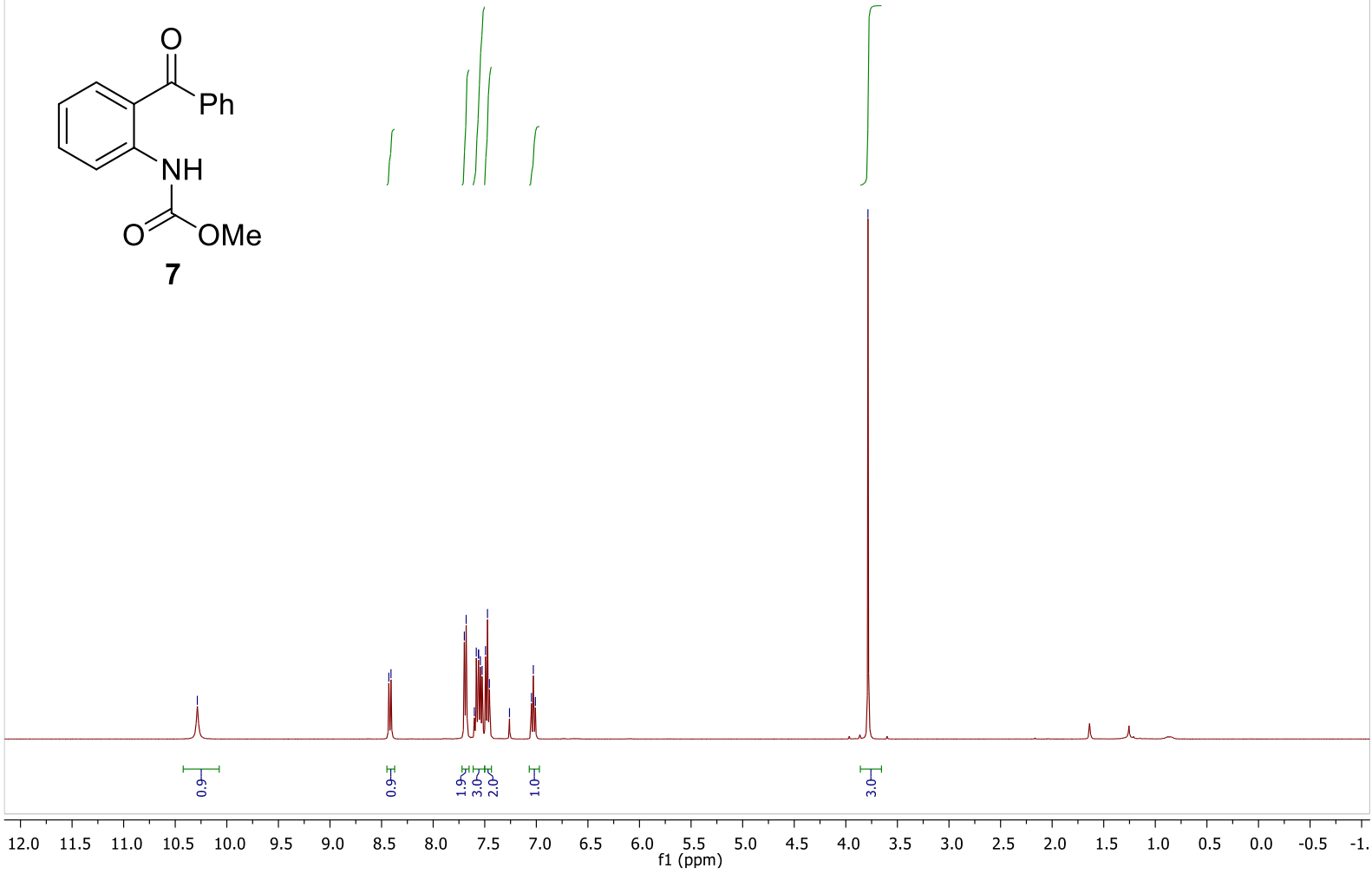

${ }^{1} \mathrm{H} \mathrm{NMR}$ in $\mathrm{CDCl}_{3}$ at $400 \mathrm{MHz}$
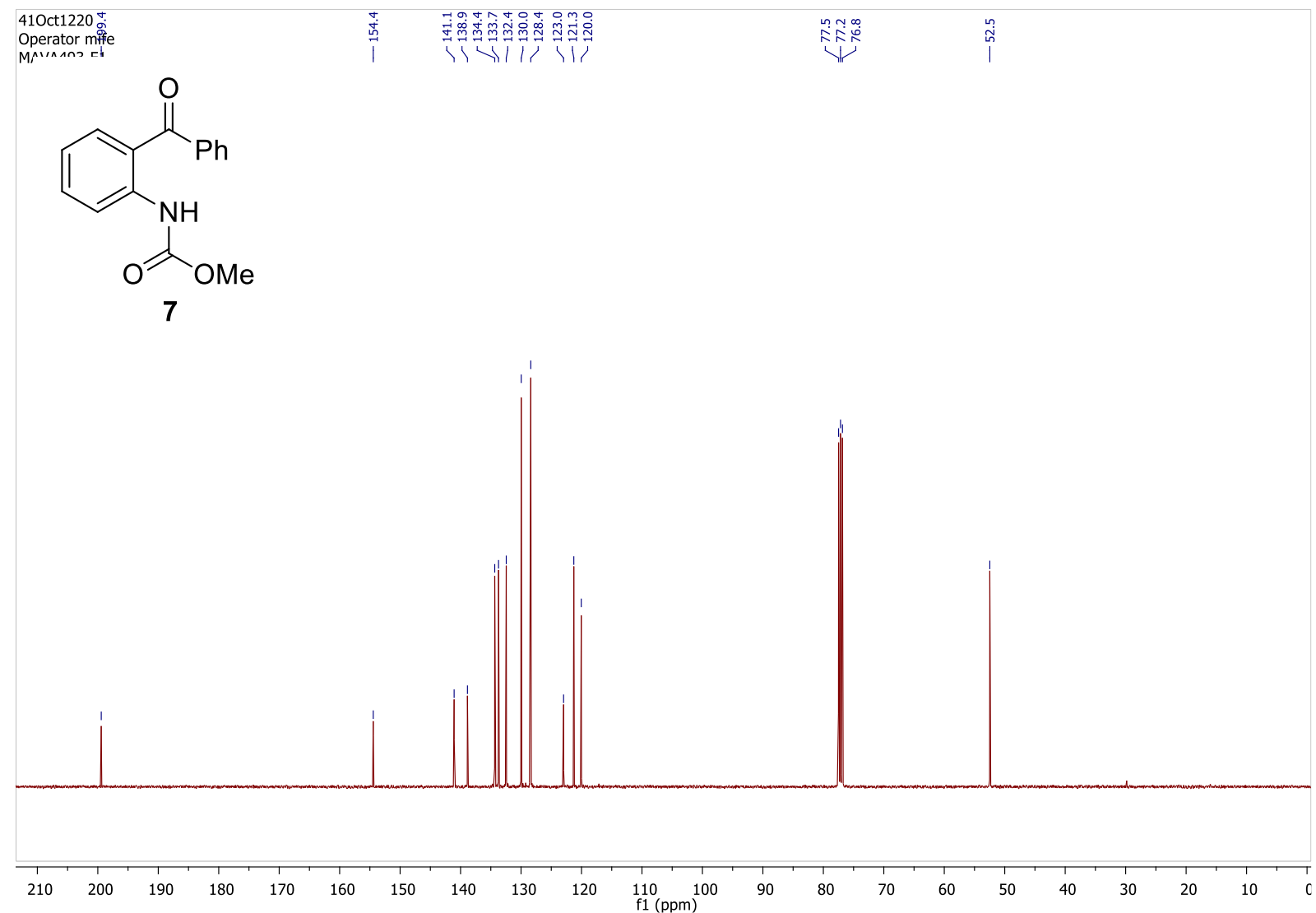

${ }^{13} \mathrm{C}\left\{{ }^{1} \mathrm{H}\right\}$ NMR in $\mathrm{CDCl}_{3}$ at $100 \mathrm{MHz}$ 


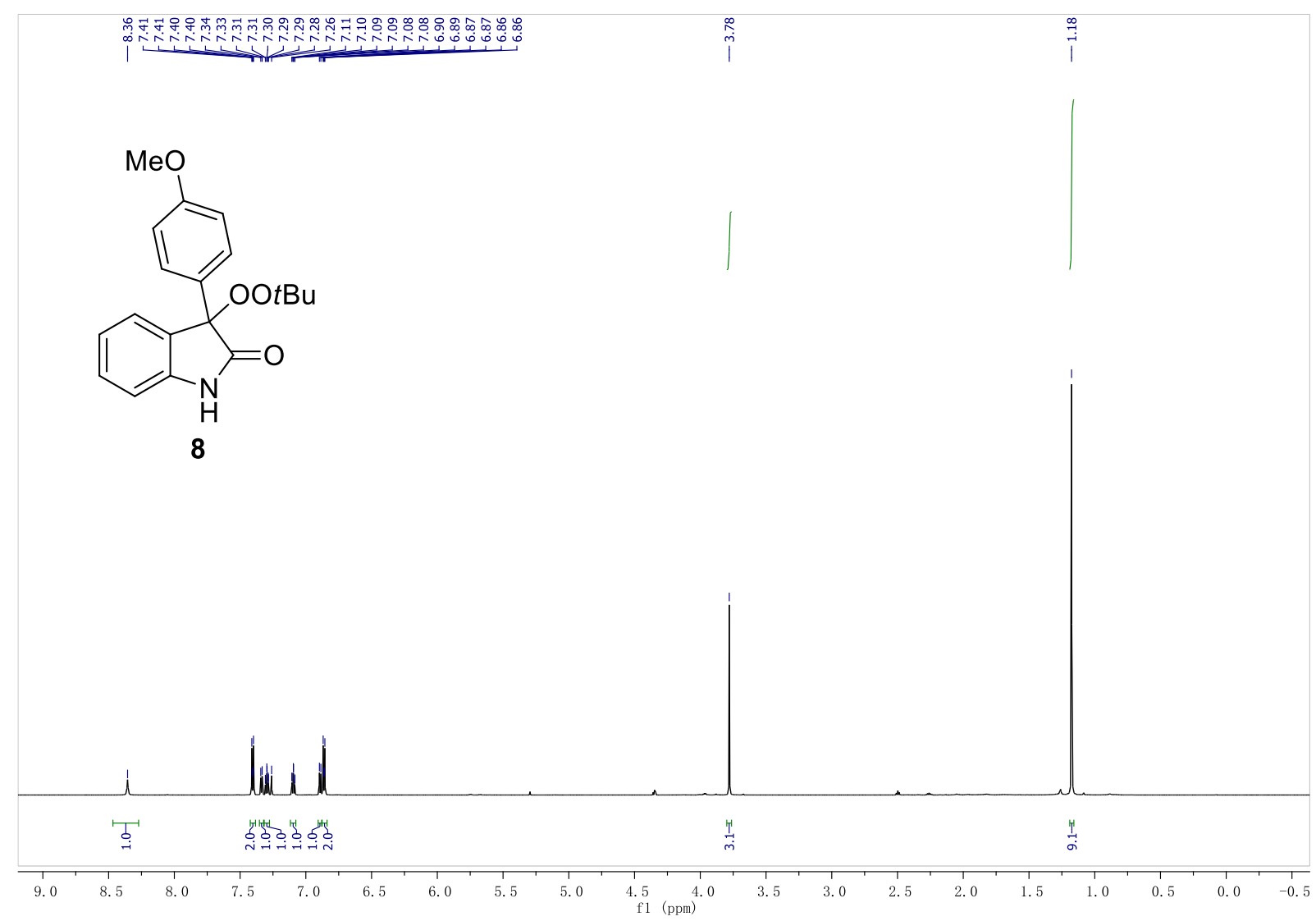

${ }^{1} \mathrm{H} \mathrm{NMR}$ in $\mathrm{CDCl}_{3}$ at $700 \mathrm{MHz}$

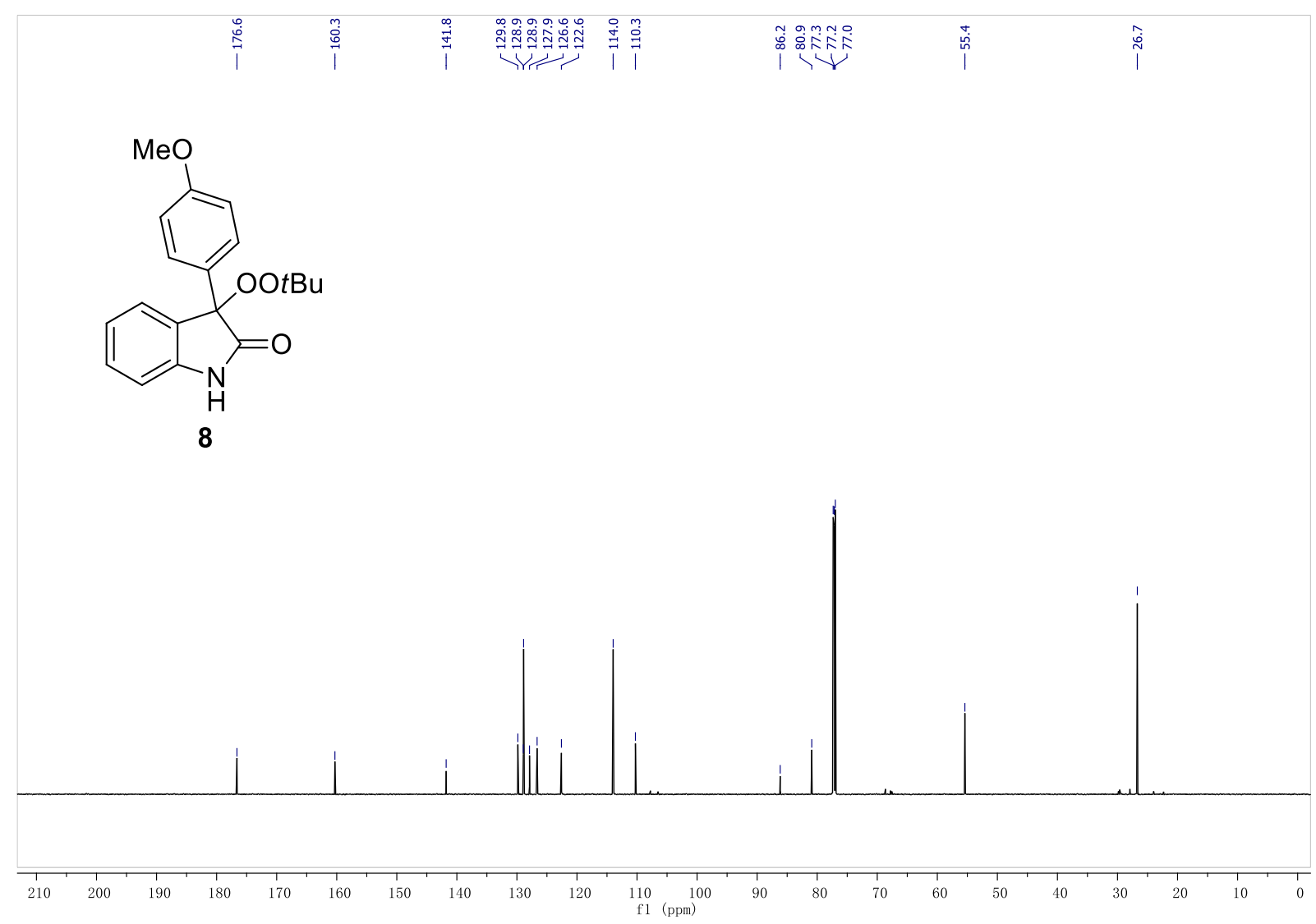


<smiles>CC(C)(C)c1cc(CC2(c3ccccc3)OC(=O)Nc3ccccc32)cc(C(C)(C)C)c1O</smiles>

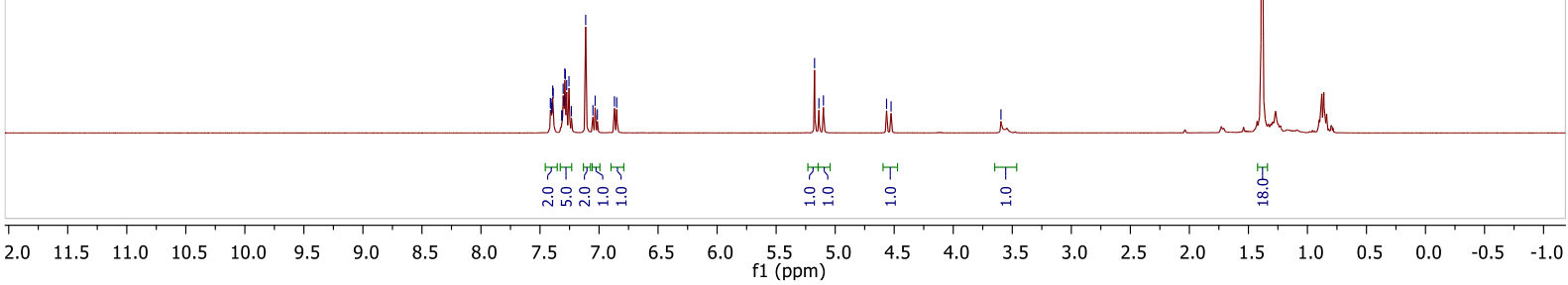

${ }^{1} \mathrm{H} \mathrm{NMR}$ in $\mathrm{CDCl}_{3}$ at $400 \mathrm{MHz}$

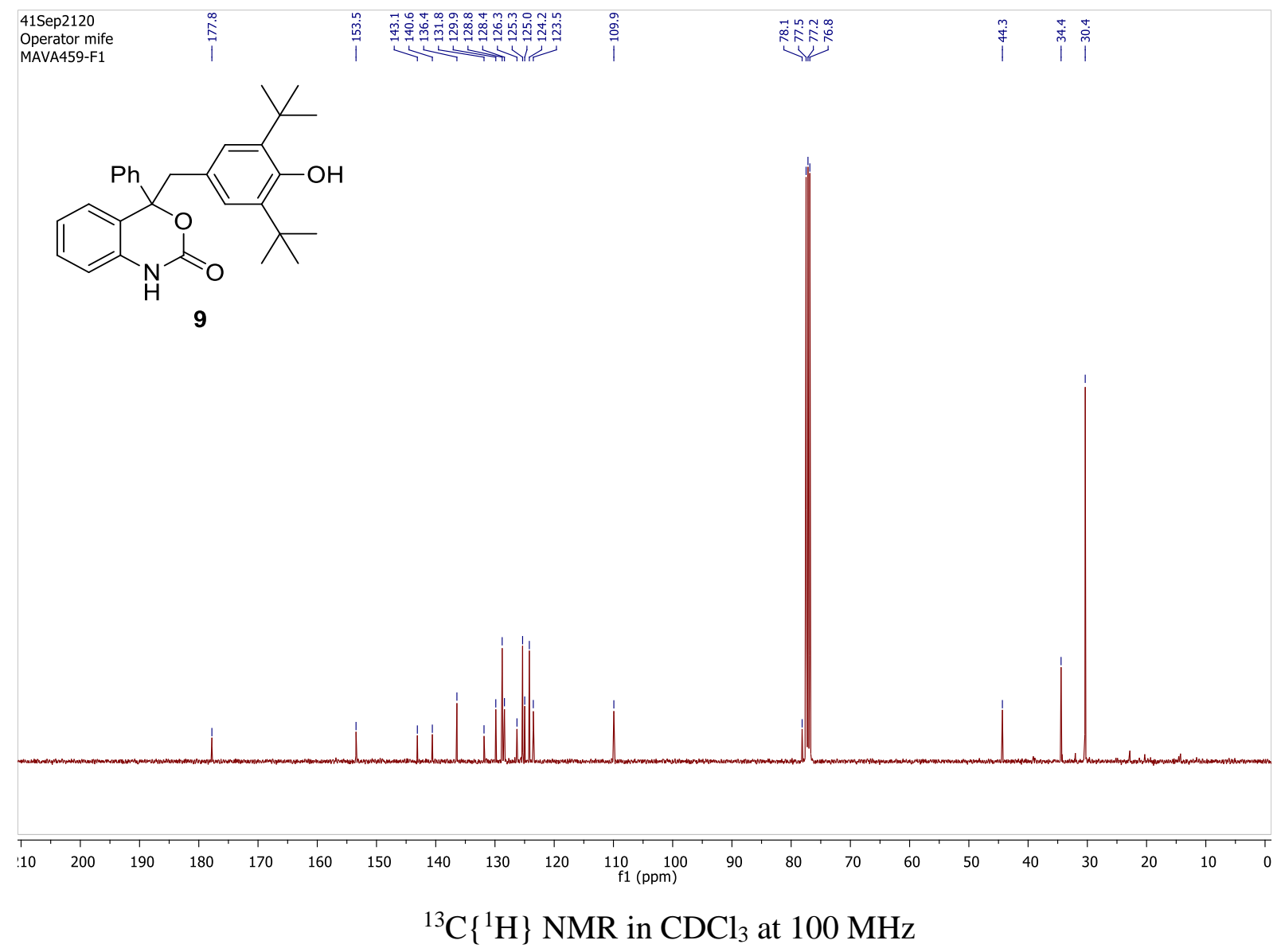




\section{References}

${ }^{1}$ Ma, S.; Han, X.; Krishnan, S.; Virgil, S. C.; Stoltz, B. M. Catalytic Enantioselective Stereoablative Alkylation of 3-Halooxindoles: Facile Access to Oxindoles with C3 All-Carbon Quaternary Stereocenters. Angew. Chem. Int. Ed. 2009, 48, 8037-8041.

${ }^{2}$ Toullec, P. Y.; Jagt, R. B. C.; de Vries, J. G.; Feringa, B. L.; Minnaard, A. J. Rhodium-Catalyzed Addition of Arylboronic Acids to Isatins: An Entry to Diversity in 3-Aryl-3-Hydroxyoxindoles. Org. Lett. 2006, 8, 2715-2718.

${ }^{3}$ Gade, A. B.; Bagle, P. N.; Shinde, P. S.; Bhardwaj, V.; Banerjee, S.; Chande, A.; Patil, N. T. Catalytic Enantioselective 1,3-Alkyl Shift in Alkyl Aryl Ethers: Efficient Synthesis of Optically Active 3,3'-Diaryloxindoles. Angew. Chem. Int. Ed. 2018, 57, 5735-5739.

${ }^{4}$ Xiao, Z. -K.; Yin, H. -Y.; Shao, L. -X. N-Heterocyclic Carbene-Palladium(II)-1-Methylimidazole Complex Catalyzed $\alpha$-Arylation of Oxindoles with Aryl Chlorides and Aerobic Oxidation of the Products in a One-Pot Procedure. Org. Lett. 2013, 15, 1254-1257.

${ }^{5}$ Ghosh, S.; Kinthada, L. K.; Bhunia, S.; Bisai, A. Lewis acid-catalyzed Friedel-Crafts alkylations of 3-hydroxy-2-oxindole: an efficient approach to the core structure of azonazine. Chem. Commun. 2012, $48,10132-10134$.

${ }^{6}$ Chaudhari, M. B.; Sutar, Y.; Malpathak, S.; Hazra, A.; Gnanaprakasam, B. Transition-Metal-Free CH Hydroxylation of Carbonyl Compounds. Org. Lett. 2017, 19, 3628-3631.

7 Yin, L.; Kanai, M.; Shibasaki, M. A Facile Pathway to Enantiomerically Enriched 3-Hydroxy-2Oxindoles: Asymmetric Intramolecular Arylation of $\alpha$-Keto Amides Catalyzed by a PalladiumDifluorPhos Complex. Angew. Chem. Int. Ed. 2011, 50, 7620-7623.

8 Verschueren, K.; Cobbaut, M.; Demaerel, J.; Saadah, L.; Voet, A. R. D.; Van Lint, J.; De Borggraeve, W. M. Discovery of a potent protein kinase D inhibitor: insights in the binding mode of pyrazolo[3,4-d]pyrimidine analogues. Med. Chem. Commun. 2017, 8, 640-646.

9 Parrick, J.; Yahya, A.; Ijaz, A. S.; Yizun, J. Convenient preparation of 3,3-dibromo-1,3dihydroindol-2-ones and indole-2,3-diones (isatins) from indoles. J. Chem. Soc., Perkin Trans. 1 1989, 2009-2015.

${ }^{10}$ Klare, H. F. T.; Goldberg, A. F. G.; Duquette, D. C.; Stoltz, B. M. Oxidative Fragmentations and Skeletal Rearrangements of Oxindole Derivatives. Org. Lett. 2017, 19, 988-991.

${ }^{11}$ Bruker SAINT v8.38B Copyright @ 2005-2019 Bruker AXS.

${ }^{12}$ Sheldrick, G. M. (1996). SADABS. University of Göttingen, Germany.

${ }^{13}$ Dolomanov, O. V.; Bourhis, L. J.; Gildea, R. J.; Howard, J. A. K.; Puschmann, H. OLEX2, J. Appl. Cryst. 2009, 42, 339-341.

${ }^{14}$ Huebschle, C. B.; Sheldrick, G. M.; Dittrich, B. ShelXle: a Qt graphical user interface for SHELXL, J. Appl. Cryst. 2011, 44, 1281-1284.

${ }^{15}$ Sheldrick, G. M. (2015). SHELXS v 2016/4 University of Göttingen, Germany.

${ }^{16}$ Spek, A. L. Structure validation in chemical crystallography. Acta Cryst. 2009, D65, 148-155. 